\title{
OPTICAL RESONANCES \\ IN MULTILAYER STRUCTURES
}


Optical resonances in multilayer structures,

Ph.D. thesis, University of Twente, The Netherlands

The research presented in this dissertation was carried out at the group of Applied Analysis and Mathematical Physics, Faculty of Electrical Engineering, Mathematics and Computer Science, and at the MESA+ Institute for Nanotechnology, University of Twente, Enschede, The Netherlands.

This research was supported by NanoNed, a national nanotechnology program coordinated by the Dutch Ministry of Economic Affairs.

Samenstelling promotiecommissie

Voorzitter en secretaris:

prof. dr. ir. A. J. Mouthaan, University of Twente

Promotor:

prof. dr. ir. E. W. C. van Groesen, University of Twente

Ass. Promotor:

dr. M. Hammer, University of Twente

Referent:

prof. dr. B. J. Hoenders, RU Groningen

Deskundige:

dr. Z. Jakšić, University of Belgrade

Leden:

prof. dr. H. J. W. M. Hoekstra, University of Twente

prof. dr. L. Kuipers, University of Twente

prof. dr. H. P. Urbach, TU Delft

Copyright 2008 by Milan Maksimović, Enschede, The Netherlands

ISBN 978-90-365-2657-9 


\title{
OPTICAL RESONANCES \\ IN MULTILAYER STRUCTURES
}

\author{
DISSERTATION
}

to obtain

the degree of doctor at the University of Twente, on the authority of the rector magnificus, prof. dr. W. H. M. Zijm,

on account of the decision of the graduation committee, to be publicly defended

on Friday, 11 April 2008 at 16:45

by

Milan Maksimović

born on 6 April 1976

in Sremska Mitrovica, Serbia. 
The dissertation is approved by

the promotor

prof. dr. E. van Groesen

the assistant promotor

dr. M. Hammer 
This thesis is dedicated to Sanja 



\section{Contents}

1 Introduction $\quad 7$

1.1 Maxwell equations and electromagnetic fields . . . . . . . . . 8

1.1.1 Materials and constitutive relations . . . . . . . . 9

1.1.2 Interface conditions . . . . . . . . . . . . . . 11

1.1.3 Poynting theorem and energy conservation . . . . . . . 12

1.1.4 Wave equation . . . . . . . . . . . . 13

1.2 Wave propagation in one-dimensional optical systems . . . . . . 14

1.2.1 Plane waves in a linear, homogeneous isotropic media . . 14

1.2.2 Scattering problems and transfer matrix method . . . . . 15

1.2.3 Periodic multilayers . . . . . . . . . . . . . 18

1.2.4 Periodic multilayer with defects . . . . . . . . . 21

1.2.5 Deterministic non-periodic multilayers . . . . . . . . 21

1.3 Open structures and quasi-normal modes . . . . . . . . . . . 22

1.4 Negative index metamaterials . . . . . . . . . . . . . . . . . 24

1.5 Thermal radiation and multilayer structures . . . . . . . . 25

1.6 Outline of the thesis . . . . . . . . . . . . . . 26

2 Resonances and quasi-normal modes 29

2.1 Quasi-normal modes and multilayers . . . . . . . . . . . . 32

2.1.1 Resonances and QNMs of single dielectric slab . . . . . . 32

2.1.2 QNMs and defect resonances in multilayers . . . . . . . 33

2.2 Two-component formalism and QNM expansion . . . . . . . . 36

2.2.1 Pseudo-inner product, orthogonality and completeness . . 37

2.2.2 QNM expansion and exponentially decaying fields . . . 40

2.2.3 QNM expansion and the transmission problems ..... . 41

2.3 Time-independent perturbation theory for QNMs . . . . . . . . 46

2.3.1 QNMs perturbation theory and two-component formalism 47

2.3.2 Variational QNM perturbation theory . . . . . . . . 48

3 Field representation for optical defect resonances in multilayer microcavities using quasi-normal modes $\quad 51$

3.1 Introduction . . . . . . . . . . . . . . 52

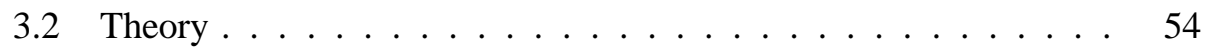


3.2.1 Solutions by transfer-matrix method . . . . . . . . . 55

3.2.2 Field template and variational formulation for transmittance problem . . . . . . . . . . . . 57

3.3 Results and Discussion . . . . . . . . . . . . . . . 59

3.3.1 Symmetric single cavity structure . . . . . . . . . 59

3.3.2 Asymmetric single cavity . . . . . . . . . . . . 62

3.3.3 Double cavity structure . . . . . . . . . . . . 62

3.3.4 Multiple cavity structure with flat-top narrow-band transmission . . . . . . . . . . . . . . . 64

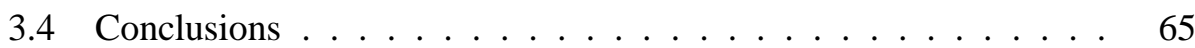

4 Coupled optical defect microcavities in 1D photonic crystals and quasinormal modes $\quad 69$

4.1 Introduction . . . . . . . . . . . . . . 70

4.2 Theory . . . . . . . . . . . . . . . . . 71

4.2 .1 Coupled cavities . . . . . . . . . . . . . 72

4.2.2 First order perturbation correction for complex eigenfrequencies . . . . . . . . . . . . . . . 74

4.3 Results and Discussion . . . . . . . . . . . . . . . 75

4.3.1 Double cavity structure . . . . . . . . . . . 75

4.3.2 Multiple cavity structures . . . . . . . . . . 79

4.4 Conclusions . . . . . . . . . . . . . . . 82

4.5 Appendix A: Transfer matrix method . . . . . . . . . . . . 83

4.6 Appendix B: Variational QNM model of the transmission problem 84

5 Negative index metamaterials and thermal radiation $\quad 87$

5.1 Negative index metamaterials . . . . . . . . . . . . . 88

5.1.1 Permeability, permittivity and refractive index . . . . . 89

5.1.2 Energy density, phase and group velocity in NIM . . . . . 92

5.1.3 Photon momentum in NIM . . . . . . . . . . . . . . 93

5.1.4 Snell's law and negative refraction . . . . . . . . . . . 94

5.2 Multilayers containing negative index metamaterials . . . . . . 95

5.2.1 Phase compensation effect . . . . . . . . . 95

5.2.2 Periodic structures with NIMs and non-Bragg bandgaps . 96

5.2.3 Transmission spectra of finite multilayers with NIM . . 98

5.3 Thermal radiation and NIM materials . . . . . . . . . . . 99

5.3.1 Blackbody radiation and Planck's law . . . . . . . . . . . 100

5.3.2 Kirchhoff's law for thermal radiation . . . . . . . . . . 101

5.3.3 Thermal radiation antennas with multilayer structures . . . 102

6 Transmission spectra of Thue-Morse multilayers containing negative $\begin{array}{lr}\text { index metamaterials } & 105\end{array}$

6.1 Introduction . . . . . . . . . . . . . . . 106

6.2 Theory and results . . . . . . . . . . . . . . 106 
6.3 Concluding remarks . . . . . . . . . . . . . . . . . . . . 109

7 Thermal radiation and 1D periodic structures containing negative index metamaterials

7.1 Introduction . . . . . . . . . . . . . . . . . . . . . . 112

7.2 The Planck law in negative index metamaterials . . . . . . . . . 113

7.3 Thermal radiation and multilayers containing negative index metamaterials . . . . . . . . . . . . . . . . . . . 114

7.4 Results and discussion . . . . . . . . . . . . . . . . . 116

7.5 Concluding remarks . . . . . . . . . . . . . . . . . . . . 119

8 Emittance tailoring by Cantor multilayers containing negative index metamaterial

8.1 Introduction . . . . . . . . . . . . . . . . . . . . . 122

8.2 Theory . . . . . . . . . . . . . . . . . . 123

8.3 Results and discussion . . . . . . . . . . . . . . . . . 127

8.4 Concluding remarks . . . . . . . . . . . . . . . . . 134

$\begin{array}{ll}\text { Bibliography } & 137\end{array}$

$\begin{array}{ll}\text { List of publications } & 148\end{array}$

$\begin{array}{lr}\text { Summary and outlook } & 151\end{array}$

$\begin{array}{ll}\text { Samenvatting } & 154\end{array}$

$\begin{array}{lr}\text { Acknowledgment } & 159\end{array}$ 


\section{Chapter 1}

\section{Introduction}

\section{ABSTRACT}

This chapter briefly reviews parts of the classical electrodynamics necessary for studying a wave propagation propagation in multilayer structures. In the end of the chapter outline of the thesis is given. 
Optics is the branch of physics that describes the phenomena associated with the propagation of light and the interaction of light with matter. According to the macroscopic, classical Maxwell theory light is an electromagnetic field [1,2]. Microscopic interaction of light with matter, at a more fundamental level is studied in quantum optics [3] that replaces the classical theory for specific purposes. However, a very broad range of phenomena in the macroscopic world and many problems of practical interest can be addressed in the framework of classical electrodynamics [2].

The field of optics usually deals with the behavior of visible, infrared, and ultraviolet light. Results and concepts obtainable for frequency ranges can be transferred to other parts of the spectrum, depending on the available material properties for these frequencies and an technological aspects. Therefore, general models that can be used in any frequency range to describe phenomena and specific devices are of particular interest.

Multilayer structures, that are periodical in their optical properties in one direction, have been known for a long time and represents more than a century old subject of investigation [1,4]. Most common applications are efficient Bragg mirrors and various filter structures, which are standard parts of many optical systems $[5,6]$.

In recent years, artificial structures with spatial periodicity in more than one dimension became popular. These structures known as Photonic Crystals can create frequency ranges in which propagation of light is prohibited [7, 8, 9]. Ideally, full 3D structures suppress light propagation for all polarizations and directions.

Nevertheless, both fundamental and applied research in multilayer optics is still important due to relevance of multilayer structures for optical systems. By the introduction of specific defects in otherwise periodic configurations one can very effectively engineer the optical transmission properties. Present research efforts are directed towards the exploration and utilization as resonant cavities in applications such as lasers, light-emitting diodes, channel drop filters, etc [6, 7, 4, 10, 11]. Also, knowledge gained from an investigation of multilayer structures may serve as a basis for the interpretation and the qualitative (sometimes even quantitative) understanding of higher dimensional photonic crystal structures.

\subsection{Maxwell equations and electromagnetic fields}

The electromagnetic field is fully described by the Maxwell equations, accompanied by appropriate constitutive relations and boundary conditions [1]. The time dependent Maxwell equations read

$$
\begin{gathered}
\nabla \times \mathbf{E}=-\frac{\partial \mathbf{B}}{\partial t}, \\
\nabla \times \mathbf{H}=\frac{\partial \mathbf{D}}{\partial t}+\mathbf{J}
\end{gathered}
$$




$$
\begin{aligned}
& \nabla \cdot \mathbf{D}=\rho, \\
& \nabla \cdot \mathbf{B}=0,
\end{aligned}
$$

where $\mathbf{E}$ is the electric field, $\mathbf{H}$ the magnetic field, $\mathbf{D}$ the electric displacement, $\mathbf{B}$ the magnetic induction, $\rho$ the free charge density and $\mathbf{J}$ the current density. These coupled equations describe all macroscopic electromagnetic phenomena where the primary sources of the electromagnetic fields are free charges and currents.

For wave propagation phenomena considered in optics, media without free charges and conduction currents are most relevant. With $\rho=0$ and $\mathbf{J}=0$, the Maxwell equations become homogeneous

$$
\begin{aligned}
\nabla \times \mathbf{E} & =-\frac{\partial \mathbf{B}}{\partial t}, \\
\nabla \times \mathbf{H} & =\frac{\partial \mathbf{D}}{\partial t}, \\
\nabla \cdot \mathbf{D} & =0, \\
\nabla \cdot \mathbf{B} & =0 .
\end{aligned}
$$

In this thesis we are dealing with electromagnetic waves, a special type of solutions of the Maxwell equations. These time-varying electromagnetic fields carry energy and are decoupled from the primary sources. Among all possible time-dependencies we are considering time-harmonic solutions, where all fields are of the form $\mathbf{A}(\mathbf{r}, t)=\operatorname{Re}\left\{\mathbf{A}(\mathbf{r}) e^{-i \omega t}\right\}$, for frequency $\omega$. The source free frequency domain Maxwell equations are

$$
\begin{gathered}
\nabla \times \mathbf{E}=i \omega \mathbf{B}, \\
\nabla \times \mathbf{H}=-i \omega \mathbf{D}, \\
\nabla \cdot \mathbf{D}=0, \\
\nabla \cdot \mathbf{B}=0 .
\end{gathered}
$$

To complete the description of the electromagnetic system additional constitutive relations for the field quantities $\mathbf{E}, \mathbf{H}, \mathbf{D}, \mathbf{B}$ must be specified to incorporate the material properties.

\subsubsection{Materials and constitutive relations}

A set of constitutive relations is required to complete the Maxwell equations. In general the constitutive relations involve a set of constitutive parameters and a set of constitutive operators. The constitutive parameters may be as simple as constants or they may be tensors, while the constitutive operators may be linear and integrodifferential or may involve nonlinear operations on the fields $[1,2,12]$.

If the constitutive parameters are spatially constant within a certain region of space, the medium is said to be homogeneous within that region, when this is 
not the case the medium is inhomogeneous. When the constitutive parameters are constant with time the medium is said to be stationary and if they are timedependent, the medium is non-stationary.

In the case of constitutive operators that involve time derivatives or integrals, the medium is said to be temporally dispersive, while in case of space derivatives or integrals involved, the medium is spatially dispersive. Moreover, the constitutive parameters may be dependent on other physical properties of the material, such as temperature, mechanical stress, etc.

In general, the constitutive parameters may be anisotropic and thus have to be expressed as tensors $[1,2,12]$. We address only isotropic materials, with scalar constitutive parameters, permeability and permittivity.

In vacuum the constitutive relations are simply

$$
\mathbf{D}=\epsilon_{0} \mathbf{E}, \text { and } \mathbf{B}=\mu_{0} \mathbf{H},
$$

where $\epsilon_{0}=8.854 \cdot 10^{-12} \mathrm{~F} / \mathrm{m}$ and $\mu_{0}=4 \pi \cdot 10^{-7} \mathrm{~A} / \mathrm{m}$ are the free space permittivity and permeability. These two fundamental physical constants are related to the speed of light $c=1 / \sqrt{\epsilon_{0} \mu_{0}}=2.998 \cdot 10^{8} \mathrm{~m} / \mathrm{s}$. All quantities are expressed in SI units, which is the case throughout this thesis, excluding the parts where certain quantities are normalized to become nondimensional.

Linear, isotropic and non-dispersive media are described by the constitutive relations

$$
\begin{gathered}
\mathbf{D}=\epsilon_{0} \mathbf{E}+\mathbf{P}=\epsilon_{0}\left(1+\chi_{e}\right) \mathbf{E}=\epsilon_{0} \epsilon_{r} \mathbf{E} \\
\mathbf{B}=\mu_{0} \mathbf{H}+\mathbf{M}=\mu_{0}\left(1+\chi_{m}\right) \mathbf{H}=\mu_{0} \mu_{r} \mathbf{H}
\end{gathered}
$$

where $\mathbf{P}=\epsilon_{0} \chi_{e} \mathbf{E}$ and $\mathbf{M}=\mu_{0} \chi_{m} \mathbf{H}$ are the polarization and magnetization vectors related to the electric and magnetic fields by the dimensionless electric and magnetic susceptibilities $\chi_{e}$ and $\chi_{m}$. The constants of proportionality are called relative electric permittivity $\epsilon_{r}=1+\chi_{e}$ and relative magnetic permeability $\mu_{r}=1+\chi_{m}$. They may depend on the position for inhomogeneous materials.

In the first three chapters of this thesis, we analyze structures made from dielectric materials with piecewise constant values of the permittivity $\epsilon$ in certain regions of space. They are non-magnetic with $\mu_{r}=1$ and transparent, having negligible losses in the considered frequency range. This simplified model, still covers a large range of practical situations in multilayer and integrated optics.

Further, we analyze in the subsequent parts of the thesis structures that incorporate so called negative index metamaterials. Broadly speaking, these are materials that have both negative permittivity and permeability. We consider isotropic metamaterials where permittivity and permeability remain scalar quantities with linear relationship in corresponding constitutive relations. Both losses and dispersion can be present.

Here, we outline only the general notion on a dispersive properties of the medium, i.e. a medium in which the relations between $\mathbf{D}$ and $\mathbf{E}$ as well as $\mathbf{B}$ and $\mathbf{H}$ are given by dynamic rather then instantaneous relations [2]. The physical origin of these relations is a time delay occurring between the influence of the 
electromagnetic field and the local macroscopic response of the materials in which the field exists. As a consequence a time delay exists between cause and effect, the fluxes $\mathbf{D}(t)(\mathbf{B}(t))$ are superpositions of the effects of fields $\mathbf{E}\left(t^{\prime}\right)\left(\mathbf{H}\left(t^{\prime}\right)\right)$ at all earlier times $t^{\prime}<t$. These relations are given in the form of convolutions

$$
\begin{gathered}
\mathbf{D}(\mathbf{r}, t)=\epsilon_{0}\left(\mathbf{E}(\mathbf{r}, t)+\int_{-\infty}^{t} \chi_{e}\left(\mathbf{r}, t-t^{\prime}\right) \mathbf{E}\left(\mathbf{r}, t^{\prime}\right) d t^{\prime}\right), \\
\mathbf{B}(\mathbf{r}, t)=\mu_{0}\left(\mathbf{H}(\mathbf{r}, t)+\int_{-\infty}^{t} \chi_{m}\left(\mathbf{r}, t-t^{\prime}\right) \mathbf{H}\left(\mathbf{r}, t^{\prime}\right) d t^{\prime}\right) .
\end{gathered}
$$

After Fourier transform, the frequency domain relations are

$$
\begin{aligned}
& \mathbf{D}(\mathbf{r}, \omega)=\epsilon(\mathbf{r}, \omega) \mathbf{E}(\mathbf{r}, \omega) \\
& \mathbf{B}(\mathbf{r}, \omega)=\mu(\mathbf{r}, \omega) \mathbf{H}(\mathbf{r}, \omega)
\end{aligned}
$$

where $\epsilon=\epsilon_{0}\left(1+\chi_{e}(\mathbf{r}, \omega)\right)$ and $\mu=\mu_{0}\left(1+\chi_{m}(\mathbf{r}, \omega)\right)$ are all now frequency dependent material response functions permittivity and permeability.

The principle of causality is implicit in (1.16),(1.17) because the integrals are taken up to time $t$ only, which represents the notion that the physical fields can not depend on the future state of media. A direct consequence of causality e are the Kramers-Kronig relations, i.e. the expressions that relate the real and imaginary parts of the permittivity (permeability) to each other through a Hilbert transform pair $[1,2]$. Some details on specific types of frequency dispersion are addressed in chapter 5 in connection with the description of the negative index metamaterials.

If absorption losses are present in the media, those can be modeled by complex permittivity and permeability

$$
\epsilon=\epsilon_{r e}+i \epsilon_{i m}, \text { and } \mu=\mu_{r e}+i \mu_{i m} .
$$

Here, imaginary parts arise due to induced polarizations and magnetizations associated with the presence of absorption [2].

Maxwell equations are obviously invariant with the substitution

$$
\mathbf{E} \rightarrow \mathbf{H}, \mathbf{H} \rightarrow \mathbf{E}, \epsilon \rightarrow-\mu, \mu \rightarrow-\epsilon
$$

This symmetry has an important consequence that can be quite convenient for certain types of problems.

\subsubsection{Interface conditions}

The practically most interesting problems involve situations where the material properties vary in space and have discontinuities. Then one associates the discontinuities with appropriate surfaces that separate regions in which the differential equations can be solved and the fields are well defined. Uniqueness of the solutions in adjoining regions requires a specification of the tangential fields on each 


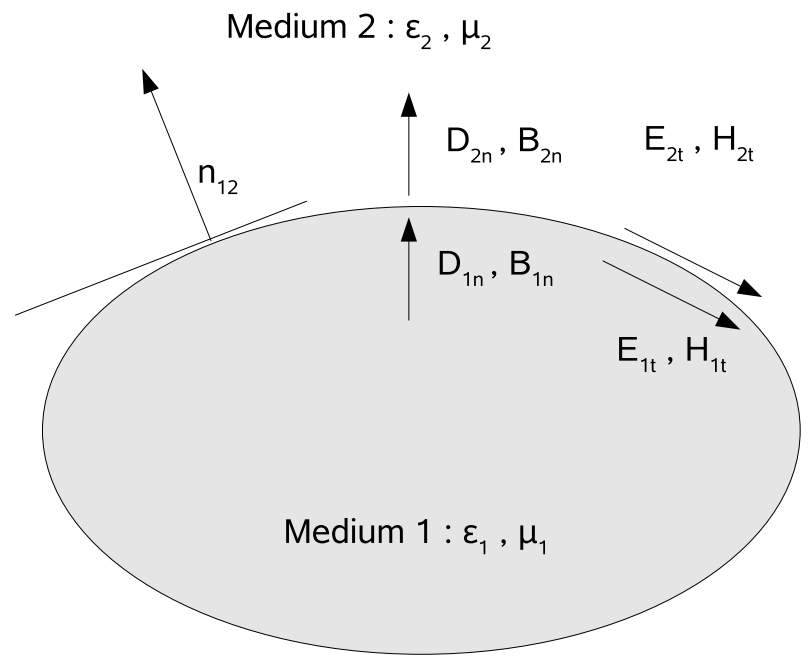

Figure 1.1: Interface conditions between two media of different material properties

side of the adjoining surface [2]. The integral form of the Maxwell equations may be used to derive interface conditions that are both physically meaningful and experimentally verifiable $[1,2]$.

For an interface between two arbitrary media without free electric charges and currents these conditions read

$$
\begin{gathered}
\mathbf{n}_{12} \times\left(\mathbf{E}_{2}-\mathbf{E}_{1}\right)=0, \\
\mathbf{n}_{12} \times\left(\mathbf{H}_{2}-\mathbf{H}_{1}\right)=0, \\
\mathbf{n}_{12} \cdot\left(\mathbf{D}_{2}-\mathbf{D}_{1}\right)=0, \\
\mathbf{n}_{12} \cdot\left(\mathbf{B}_{2}-\mathbf{B}_{1}\right)=0 .
\end{gathered}
$$

Here $\mathbf{n}_{12}$ is a unit vector normal to the interface. This is the most convenient form for the waves propagation phenomena considered in this thesis.

\subsubsection{Poynting theorem and energy conservation}

Using Maxwell equations (1.5)-(1.8) and the vector identity $\nabla \cdot(\mathbf{E} \times \mathbf{H})=\mathbf{H} \cdot \nabla \times$ $\mathbf{E}-\mathbf{E} \cdot \nabla \times \mathbf{H}$ we can derive relation

$$
\nabla \mathbf{S}+\left(\mathbf{E} \frac{\partial \mathbf{D}}{\partial t}+\mathbf{H} \frac{\partial \mathbf{B}}{\partial t}\right)=-\mathbf{J E}
$$

where

$$
\mathbf{S}=\mathbf{E} \times \mathbf{H} .
$$

is called the Poynting vector.

For linear, non-dispersive and lossless media an energy density of the electromagnetic field stored in the material can be defined as

$$
W=\frac{1}{2} \epsilon \mathbf{E} \cdot \mathbf{E}+\frac{1}{2} \mu \mathbf{H} \cdot \mathbf{H} .
$$


By evaluating the second term on the left side of (1.26), we find the relation

$$
\frac{\partial W}{\partial t}+\nabla \cdot \mathbf{S}=-\mathbf{J} \cdot \mathbf{E}
$$

which in integral form reads

$$
\frac{d}{d t} \int_{\Omega} W d \Omega+\int_{\Omega} \mathbf{J} \cdot \mathbf{E} d \Omega=-\oint_{\partial \Omega} \mathbf{S} \partial \Omega
$$

This is one form of the Poynting theorem which in this case can be interpreted as an energy conservation law: the total power entering a volume $\Omega$ through the surface $\partial \Omega$ increases the field energy inside the volume as is lost through absorption processes in the medium. In lossless and non-dispersive media the vector $\mathbf{S}$ can be interpreted as the power flux density carried by the electromagnetic wave, effectively defining the direction of the power flow across the boundary.

However, this interpretation is valid only in the case of non-dispersive materials. When dispersion and losses are present the interpretation of the stored energy in the material looses its foundation [2]. In general, the power flow interpretation of the Poynting theorem has to be carefully examined for each particular situation [2].

\subsubsection{Wave equation}

The wave equation is a second-order differential equation, obtained from the original system of coupled equations.). Taking the curl of the (1.5) and using (1.6), we obtain the second wave order equation for the electric and magnetic fields

$$
\begin{aligned}
& \mu \nabla \times \mu^{-1} \nabla \times \mathbf{E}+\epsilon \mu \frac{\partial^{2} \mathbf{E}}{\partial t^{2}}=0, \\
& \epsilon \nabla \times \epsilon^{-1} \nabla \times \mathbf{H}+\epsilon \mu \frac{\partial^{2} \mathbf{H}}{\partial t^{2}}=0 .
\end{aligned}
$$

In the frequency domain when time harmonic fields with frequency $\omega$ are considered wave equations become

$$
\begin{aligned}
& \mu \nabla \times \mu^{-1} \nabla \times \mathbf{E}-\omega^{2} \epsilon \mu \mathbf{E}=0, \\
& \epsilon \nabla \times \epsilon^{-1} \nabla \times \mathbf{H}-\omega^{2} \epsilon \mu \mathbf{H}=0 .
\end{aligned}
$$

For homogeneous media, where the electric field satisfies $\nabla \mathbf{E}=0$ equations (1.31) and (1.32) become

$$
\nabla^{2} \mathbf{E}-\epsilon \mu \frac{\partial^{2} \mathbf{E}}{\partial t^{2}}=0, \quad \nabla^{2} \mathbf{H}-\epsilon \mu \frac{\partial^{2} \mathbf{H}}{\partial t^{2}}=0
$$

In the frequency domain these become Helmholtz equations

$$
\nabla^{2} \mathbf{E}+\omega^{2} \epsilon \mu \mathbf{E}=0, \quad \nabla^{2} \mathbf{H}+\omega^{2} \epsilon \mu \mathbf{H}=0 .
$$


Frequently it is convenient to write the physical quantity $\epsilon \mu$ that appears in the wave equation in the alternative form

$$
\epsilon \mu=\frac{n^{2}}{c^{2}}
$$

where $c=1 / \sqrt{\epsilon_{0} \mu_{0}}$ is velocity of light in vacuum and $n^{2}=\epsilon_{r} \mu_{r}$ is dimensionless quantity known as the index of refraction or refractive index. Note that the refractive index represents a derived, formal construct that does not appear directly in the Maxwell equations.

Particular care is required with the definition of the (sign of) the refractive index, in cases where the permittivity and permeability are complex quantities, with not necessarily positive real parts. The sign of the square root in the expression for refractive index is determined according to the following rule:

$$
\begin{array}{ll}
\operatorname{Re}(n)<0 & \text { if } \operatorname{Re}(\epsilon)<0 \text { and } \operatorname{Re}(\mu)<0, \\
\operatorname{Re}(n) \geq 0 & \text { otherwise. }
\end{array}
$$

The term "negative index metamaterials" refers to situations where the first alternative of the rule (1.38) applies. The standard model for the absorption in the material is obtained by taking the complex form of the permittivity and permeability in corresponding materials $[1,12]$. Then, for the complex material response functions, permeability $\epsilon(\omega)=\epsilon_{r e}(\omega)+i \epsilon_{i m}(\omega)$ and permittivity $\mu(\omega)=\mu_{r e}(\omega)+i \mu_{i m}(\omega)$, the complex refractive index $n(\omega)=n_{r e}(\omega)+i n_{i m}(\omega)$ is given by [13]

$$
n=\sqrt{|\epsilon||\mu|} \exp \left(\frac{i}{2}\left[\operatorname{arccot}\left(\frac{\epsilon_{r e}}{\epsilon_{i m}}\right)+\operatorname{arccot}\left(\frac{\mu_{r e}}{\mu_{i m}}\right)\right]\right)
$$

Where, in the presence of dispersion all of quantities are understood to be frequency dependent. For more details see chapter (5) and references therein.

\subsection{Wave propagation in one-dimensional optical systems}

Central subject of this thesis are electromagnetic multilayer structures in which permittivity and permeability are spatially varying in one direction. We consider specific models and methods for solving wave propagation problems that involve general multilayer structures under external excitation by incoming waves $[4,5,8]$.

\subsubsection{Plane waves in a linear, homogeneous isotropic media}

We are interested in the general behavior of EM waves in the frequency domain, so we seek simple solutions to the homogeneous Helmholtz equation

$$
\left(\nabla^{2}+k^{2}\right) \mathbf{E}(\mathbf{r}, \omega)=0,
$$

that governs the EM fields in source-free regions of space. Here

$$
k^{2}(\omega)=\omega^{2} \epsilon(\omega) \mu(\omega)
$$


is the propagation constant. In a rectangular Cartesian coordinate system equation (1.40) reduces to three scalar equations for each component $E_{x}, E_{y}, E_{z}$ of the electric field. These equations may be solved by separation of variables; solutions of (1.40) can be presented as

$$
\mathbf{E}(\mathbf{r}, \omega)=\mathbf{E}(\omega) \mathrm{e}^{i \mathbf{k} \cdot r}
$$

for wave vector $\mathbf{k}=\left[k_{x}, k_{y}, k_{z}\right]$ and vector amplitude spectrum $\mathbf{E}(\omega)$. The wave number is the magnitude of the wave vector $|\mathbf{k}|^{2}=k^{2}=k_{x}^{2}+k_{y}^{2}+k_{z}^{2}$. Solution (1.42) represent propagating plane waves, with planes as the spatial surfaces over which the phase of the field is constant.

When lossy materials with complex permittivity and permeability are considered, the wave vector becomes complex

$$
\mathbf{k}=\mathbf{k}_{r e}+i \mathbf{k}_{i m}
$$

If the real and imaginary parts of the wave vector are collinear, (1.42) describes a uniform plane wave, otherwise the waves are nonuniform. Certain issues related to the proper determination of the complex wave vector arise when materials are dispersive and lossy. This is the case for the wave propagation in negative index metamaterials. Some specifics are discussed in chapter 5.

\subsubsection{Scattering problems and transfer matrix method}

For the propagation of the electromagnetic waves through planar layered structures made of the piecewise constant, homogeneous, and isotropic media without sources, the vectorial wave equation reduces to two uncoupled scalar equations [12]. One distinguishes two types of optical fields: For Transverse Electric (TE) waves the electric field is perpendicular to the plane spanned by the direction of propagation of the incident wave and its projection on the layer interfaces, while for Transverse Magnetic (TM) waves the magnetic field is perpendicular to that plane of incidence. Cartesian coordinates are introduced as in Figure 1.2 to describe the propagation of plane waves through the multilayer stacks, where the $x$-axis is parallel to the layer interfaces, while the $z$-axis is perpendicular to the stack surface, such that the coordinates $x$ and $z$ span the plane of incidence.

For TE waves the electric field $\mathbf{E}=\left(0, E_{y}, 0\right)$ is linearly polarized in the $y$ direction. Time-harmonic fields $\mathbf{E}(\mathbf{r}, t)=\mathbf{E}(\mathbf{r}) \mathrm{e}^{-i \omega t}$ with real angular frequency $\omega$ are considered. Then under external time harmonic, TE polarized excitation the field in the medium is described by the scalar function $E_{y}(x, z)$ (where we drop the subscript ${ }_{y}$ to simplify the notation). With this choice of polarization the Maxwell equations reduce, after stratification $\partial_{y}=0$, to the Helmholtz equation for TE waves

$$
\left(\frac{\partial^{2}}{\partial x^{2}}+\mu(z) \frac{\partial}{\partial z} \frac{1}{\mu(z)} \frac{\partial}{\partial z}+\frac{\omega^{2}}{c^{2}} \epsilon \mu\right) E(x, z)=0 .
$$




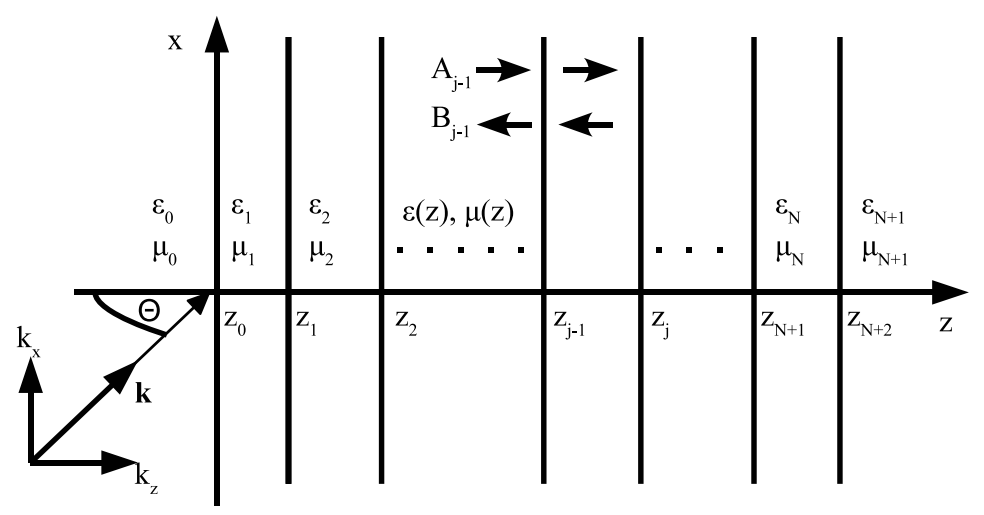

Figure 1.2: An inhomogeneous multilayer structure (i.e. a stratified medium) with piecewise constant, $z$-dependent permittivity $\epsilon$ and permeability $\mu$. The structure is invariant along the $x$ - and $y$-directions. Oblique incidence of plane electromagnetic waves is considered, with incidence angle $\theta$.

Analogously, the principal magnetic component $H$ of time harmonic TM waves with $y$-polarized magnetic field $\mathbf{H}(x, z, t)=(0, H, 0)(x, z) \mathrm{e}^{-i \omega t}$ satisfies the Helmholtz equation

$$
\left(\frac{\partial^{2}}{\partial x^{2}}+\epsilon(z) \frac{\partial}{\partial z} \frac{1}{\epsilon(z)} \frac{\partial}{\partial z}+\frac{\omega^{2}}{c^{2}} \epsilon \mu\right) H(x, z)=0
$$

Fourier transform along the layer interfaces separates the $x$ - and $z$-dependent parts of the principal fields, such that these can be represented in the form

$$
E(x, z)=E(z) \mathrm{e}^{ \pm i k_{x} x} \text {, and } H(x, z)=H(z) \mathrm{e}^{ \pm i k_{x} x} .
$$

where the $x$-component $k_{x}$ of the wave vector now plays the role of a parameter that is defined by the angle of incidence (cf. Figure 1.2). Due to the invariance in the $x$-direction equations (1.44) and (1.45) become ordinary differential equations

$$
\left(\mu(z) \frac{\partial}{\partial z} \frac{1}{\mu(z)} \frac{\partial}{\partial z}+\frac{\omega^{2}}{c^{2}} \epsilon \mu-k_{x}^{2}\right) E(z)=0,
$$

and

$$
\left(\epsilon(z) \frac{\partial}{\partial z} \frac{1}{\epsilon(z)} \frac{\partial}{\partial z}+\frac{\omega^{2}}{c^{2}} \epsilon \mu-k_{x}^{2}\right) H(z)=0 .
$$

Note that these equations are identical for positions $z$ inside the layers with constant material properties. Differences between the polarizations manifest only through different interface conditions at the boundaries between the layers. For TE waves, the quantities

$$
E, \quad \frac{1}{\mu} \frac{\partial E}{\partial z}
$$


should be continuous across the interfaces, while continuity of

$$
H, \quad \frac{1}{\epsilon} \frac{\partial H}{\partial z}
$$

is required for TM polarized waves. Note that the following steps are valid also for complex permittivity and permeability.

Analytic solutions of the Helmholtz equation for the multilayer structure of Figure 1.2 in the $j$-th layer can be written

$$
F_{j}(z)=A_{j} \mathrm{e}^{i k_{j z}\left(z-z_{j-1}\right)}+B_{j} \mathrm{e}^{-i k_{j z}\left(z-z_{j-1}\right)}
$$

where $F$ replaces the $E$-field in the case of TE polarization and the $H$-field for TM polarization. $k_{j z}$ is the $z$-component of the local wave vector in layer $j$, defined by

$$
k_{j z}^{2}=\frac{\omega^{2}}{c^{2}} \epsilon_{j} \mu_{j}-k_{x}^{2},
$$

for vacuum speed of light $c$.

We consider a situation when a plane wave $F_{0}(x, z)=A_{0} \mathrm{e}^{i k_{x} x+i k_{0 z} z}$ with given amplitude $A_{0}$ is incident onto the multilayer structure, coming from a semiinfinite, homogeneous (conventional, transparent dielectric) medium, with wave vector $\mathbf{k}_{0 j}=\left(k_{x}, 0, k_{0 z}\right)$. Its $x$ - and $z$-components $k_{x}=\left(n_{0} \omega / c\right) \sin \theta$ and $k_{0 z}=$ $\left(n_{0} \omega / c\right) \cos \theta$ define / are defined by the incidence angle $\theta$, where $n_{0}=\sqrt{\epsilon_{0} \mu_{0}}$ is the local refractive index of the input medium.

The local wave vector in $j$-th layer can be expressed as

$$
k_{j z}=\frac{\omega}{c} n_{j} \sqrt{1-\frac{n_{0}^{2} \sin ^{2} \theta}{n_{j}^{2}}}
$$

inside the layer $z \in\left[z_{j-1}, z_{j}\right]$ with local permittivity $\epsilon_{j}$ and permeability $\mu_{j}$, and the refractive index defined by $n_{j}=\sqrt{\epsilon_{j} \mu_{j}}$.

With the abbreviation $\eta_{j}=\mu_{j}$ for TE polarization and $\eta_{j}=\epsilon_{j}$ for TM waves, the continuity conditions $(1.49,1.50)$ for the interface between layers $j$ and $j+1$ can be written as

$$
F_{j}\left(z_{j}\right)=F_{j+1}\left(z_{j}\right), \text { and } \frac{1}{\eta_{j}} \frac{\partial F_{j}}{\partial z}\left(z_{j}\right)=\frac{1}{\eta_{l+1}} \frac{\partial F_{j+1}}{\partial z}\left(z_{j}\right) .
$$

These conditions lead to a system of equations that relates amplitudes in neighboring layers through the step matrix

$$
\left(\begin{array}{c}
A_{j} \\
B_{j}
\end{array}\right)=\frac{1}{2}\left(\begin{array}{cc}
\left(1+\frac{s_{j+1}}{s_{j}}\right) \mathrm{e}^{-i k_{j z} d_{j}} & \left(1-\frac{s_{j+1}}{s_{j}}\right) \mathrm{e}^{-i k_{j z} d_{j}} \\
\left.1-\frac{s_{j+1}}{s_{j}}\right) \mathrm{e}^{+i k_{j z} d_{j}} & \left(1+\frac{s_{j+1}}{s_{j}}\right) \mathrm{e}^{+i k_{j z} d_{j}}
\end{array}\right)\left(\begin{array}{c}
A_{j+1} \\
B_{j+1}
\end{array}\right)
$$

with the abbreviation $s_{j}=k_{j z} / \eta_{j}$ and where the separate propagation of the directional waves throughout the layers of thickness $d_{j}=z_{j}-z_{j-1}$ according to 
equation (1.51) has already been incorporated. Ordered multiplication of these matrices connects amplitudes in each layer of the structure. If the amplitude transfer is carried out over the full layer stack, one arrives at a system matrix of the form

$$
\left(\begin{array}{c}
A_{0} \\
r A_{0}
\end{array}\right)=\left(\begin{array}{ll}
m_{11} & m_{12} \\
m_{21} & m_{22}
\end{array}\right)\left(\begin{array}{c}
t A_{0} \\
0
\end{array}\right) .
$$

Here $r$ and $t$ are the reflection and transmission amplitude coefficients. Assuming that the input and the output regions consists of the conventional dielectric materials without absorption, we define the transmittance as the ratio of the optical output and input power [1] (intensity ratio for observation planes parallel to the layer surface)

$$
T(\omega, \theta)=\frac{n_{N+1} \cos \theta_{N+1}}{n_{0} \cos \theta_{0}}\left|\frac{1}{m_{11}}\right|^{2}
$$

and the reflectance as the ratio between the reflected and the incident power

$$
R(\omega, \theta)=\left|\frac{m_{21}}{m_{11}}\right|^{2}
$$

Here the incident angle $\theta$ is related to the angle $\theta_{N+1}$ in the output medium through the Snell's law

$$
n_{j} \sin \theta_{j}=n_{j+1} \sin \theta_{j+1},
$$

where $n_{j}$ and $\theta_{j}$ are refractive indices and (formal) angles in corresponding layers. This formal expression is valid for any type of material and even for NIMs, see chapter 5 and references therein.

According to the energy conservation law, when material are lossy, a quantity called absorptance can be defined as

$$
A=1-R(\omega, \theta)-T(\omega, \theta) .
$$

It represents the portion of the incident optical power that is absorbed by the structure and transformed, for example, to the thermal energy in the material.

\subsubsection{Periodic multilayers}

Consider an multilayer arrangement of two different materials with $\left\{\epsilon_{A}, \mu_{A}\right\}$ and $\left\{\epsilon_{B}, \mu_{B}\right\}$, denoted as $A$ and $B$ respectively as depicted in Figure 1.3, with periodic permittivity and permeability

$$
\epsilon(z+\Lambda)=\epsilon(z), \text { and } \mu(z+\Lambda)=\mu(z) .
$$

with period $\Lambda=a+b$. This is a traditional model for periodic optical structures that we will use as a basic model in this thesis [1,4]. The structure possesses discrete translational symmetry in contrast to the continuous translational symmetry of homogeneous media [8]. 


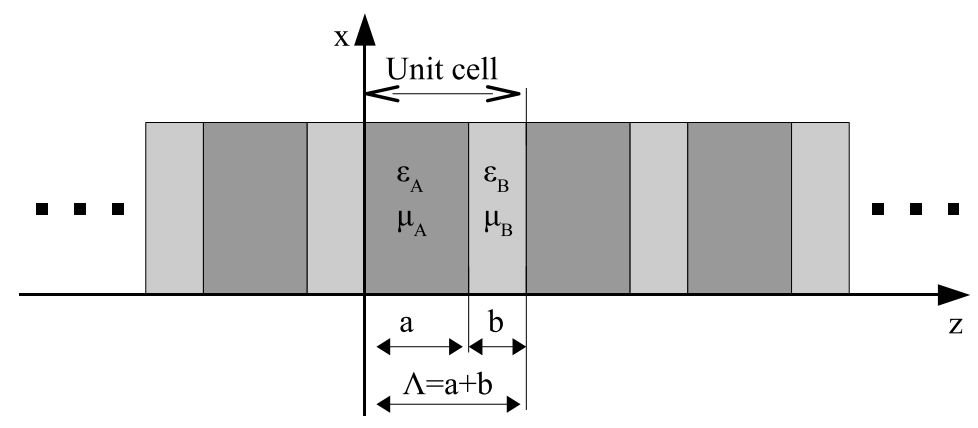

Figure 1.3: An periodic (binary) multilayer structure with piecewise constant and periodic, $z$-dependent permittivity $\epsilon$ and permeability $\mu$. The structure is invariant along the $x$ - and $y$-directions. The thicknesses of layers $A$ and $B$ are $a$ and $b$, respectively. The unit cell thickness $\Lambda$, represents period of the structure.

The wave propagation is described by equations (1.47) and (1.48) for both polarizations; the solutions are periodic according to the Bloch-Floquet theorem $[8,9,4]$. Thus, a field in the periodic multilayer can be represented in the form

$$
F(z+\Lambda)=e^{i K_{B} \Lambda} F(z) .
$$

where $K_{B}$ is the Bloch's wave number. The transfer matrix (1.55) connects amplitudes in the adjacent layers

$$
\left(\begin{array}{c}
A_{j} \\
B_{j}
\end{array}\right)=\left(\begin{array}{ll}
T_{11}^{(j)} & T_{12}^{(j)} \\
T_{21}^{(j)} & T_{22}^{(j)}
\end{array}\right)\left(\begin{array}{c}
A_{j+1} \\
B_{j+1}
\end{array}\right)
$$

while local amplitudes separated by one period are related as

$$
\left(\begin{array}{c}
A_{j} \\
B_{j}
\end{array}\right)=\left(\begin{array}{ll}
T_{11}^{(j)} & T_{12}^{(j)} \\
T_{21}^{(j)} & T_{22}^{(j)}
\end{array}\right)\left(\begin{array}{ll}
T_{11}^{(j+1)} & T_{12}^{(j+1)} \\
T_{21}^{(j+1)} & T_{22}^{(j+1)}
\end{array}\right)\left(\begin{array}{c}
A_{j+2} \\
B_{j+2}
\end{array}\right),
$$

owing to the Bloch-Floquet theorem and equation (1.62), as

$$
\left(\begin{array}{c}
A_{j} \\
B_{j}
\end{array}\right)=e^{-i K_{B} \Lambda}\left(\begin{array}{c}
A_{j+2} \\
B_{j+2}
\end{array}\right) \text {. }
$$

Due to the periodicity the amplitudes in the $(j)$-th and the $(j+2)$-nd layer are the same and equation (1.65) can be written as the homogeneous system

$$
\left(\begin{array}{cc}
T_{11}-e^{i K_{B} \Lambda} & T_{12} \\
T_{21} & T_{22}-e^{i K_{B} \Lambda}
\end{array}\right)\left(\begin{array}{c}
A_{j+2} \\
B_{j+2}
\end{array}\right)=\left(\begin{array}{l}
0 \\
0
\end{array}\right) .
$$

Nontrivial solution exits only if determinant of the system matrix $\mathbf{T}=\mathbf{T}^{j} \mathbf{T}^{j=1}$ is identical to zero:

$$
T_{11} T_{22}-T_{12} T_{21}-e^{i K_{B} \Lambda}\left(T_{11}+T_{22}\right)+e^{i 2 K_{B} \Lambda}=0 .
$$


The determinant of the unit cell transfer matrix is $\operatorname{det}(\mathbf{T})=1$ which can be seen by examining the relation

$$
\operatorname{det} \mathbf{T}=T_{11} T_{22}-T_{12} T_{21}=\operatorname{det} \mathbf{T}^{(j)} \operatorname{det} \mathbf{T}^{(j+1)} .
$$

Using the form (1.55) of the transfer matrix it follows that

$$
\operatorname{det} \mathbf{T}^{(j)}=\frac{s_{j+1}}{s_{j}}, \text { and } \operatorname{det} \mathbf{T}^{(j+1)}=\frac{s_{j+2}}{s_{j+1}} .
$$

which leads to

$$
\operatorname{det} \mathbf{T}=\frac{s_{j+1}}{s_{j}} \frac{s_{j+2}}{s_{j}}=1,
$$

where the condition $s_{j+2}=s_{j}$ holds due to the periodicity. Finally, equation (1.67) simplifies to

$$
\cos \left(K_{B} \Lambda\right)=\frac{1}{2}\left(T_{11}+T_{22}\right)=\frac{1}{2} \operatorname{tr} \mathbf{T} .
$$

Equation (1.71) connects values of the Bloch wave vector and frequency of the field through so called dispersion relation

$$
\omega=\omega\left(K_{B}, k_{x}\right)
$$

If all material properties, permeability and permittivity are real, then $K_{B} \in \mathbb{R}$, for given frequency $\omega \in \mathbb{R}$ if and only if $\left|\cos \left(K_{B} \Lambda\right)\right|<1$. Then waves can propagate in the medium without attenuation. A range of frequencies where this is satisfied is called the pass-band or the transparency band. On the other hand there may be range of frequencies for given structure where $\left|\cos \left(K_{B} \Lambda\right)\right|>1$, depending on the right-hand side of (1.71). Then solution of the (1.71) for $\omega \in R$ are characterized by complex valued Bloch wave vector $K_{B} \in \mathbb{C}$. These ranges of frequencies where propagating waves are forbidden are called the bandgaps or the stop-bands.

In fact, the suppression of wave propagation for some range of frequencies is an intrinsic property of all periodic media. Electromagnetic waves in periodic media with a frequency in to the bandgap are of the evanescent type, i.e waves exponentially attenuate in amplitude while propagating through medium. In contrast to these evanescent (bandgap) waves, propagating waves sometimes are named extended, due to fact that the energy of the waves is distributed over whole structure. An analogy with the electronic band structure in solid state physics arises and the name Photonic Crystals follows form it [8].

Equation (1.71) can be applied to the analysis of more complex unit cell's (e.g. with more then two layers in the unit cell and in arbitrary arrangement) by considering the trace of the corresponding transfer matrix [8]. Periodic repetition of the complex unit cell gives rise to the bandgap structure. Sometimes this method, called supercell method, is used for the analysis of finite (non-periodic) structures where the assumption is made that the artificial periodization does not change the optical response substantially [7,9]. 
Another approach to show the physical origin of the bandgap phenomena is of the multiple scattering description of the wave propagation [1],[12],[8]. It amounts to identifying conditions under which waves interfere constructively or destructively in such a way to support or reject wave propagation for certain frequencies. Although, this point of view is physically and intuitively very appealing, it is not easily tractable in general [12],[8].

\subsubsection{Periodic multilayer with defects}

Looking at periodic media from a symmetry point of view, the bandgap may be seen to arise from the discrete translational symmetry of the periodic media [8]. As it turns out, for the frequencies inside the bandgap wave propagation is suppressed and all waves are of the evanescent type. However, breaking the symmetry of the periodic media may give rise to specific types of propagating waves with the frequencies belonging to the bandgap range. A common way of breaking the translational symmetry is to locally change the thickness or the material properties in specific layer [8]. The emerging periodic parts of the Photonic Crystal enclosing the defect site act as frequency selective mirrors for Fabry-Perot type resonator formed by the defect layer. With a suitable adjustment of the defect parameters, a so called defect modes may be supported by the structure. These are localized states with concentration of the energy in the vicinity of the defect in contrast to the extended states of the pass bands in the periodic structure. They possess real Bloch wave vector in the frequency range of the bandgap of the underlying periodic structure $[4,8,14]$.

In this thesis, we are interested in the characterization and utilization of these defect resonances arising in finite structures. They are revealed as transmission resonances, i.e. high values of transmittance with the frequencies of the maximum of the transmittance belonging to the bandgap.

\subsubsection{Deterministic non-periodic multilayers}

The studies of the wave propagation in multilayers in general regard two different extremes: perfectly periodic media (such as photonic crystals) and absolutely random multilayers. However, there are structures that behave much like disordered ones, but are constructed according to a deterministic procedure. These are called non-periodic deterministic (NPD) media. They possess the properties of both periodic and random structures and also have some distinct features not found in periodic structures $[15,16,17]$.

Several classes distinguish themselves, depending on the algorithm used for the stack construction. The first class, called substitutional lattices is generated via a repeated substitution rule. The second large class represents NPD multilayers that are fractal by themselves. They are called multilayer fractal structures because they are constructed according to a known fractal generation algorithm [18]. This algorithm has to be stopped at some point in order to get a finite structure. 
Therefore, any structure obtained in this way is not a genuine fractal, but rather a one-dimensional pre-fractal.

The spectral transmission and reflection properties of quasi-periodic and fractal structures were widely studied in conjunction with the topics such as quasicrystals, electronic superlatices and optical multilayers $[15,16]$. Some of these specially designed multilayers have statistically self-similar optical transmission spectra and the frequencies of the resonance peaks form a fractal set $[15,16]$. Optical multilayers are specifically interesting for studying classical wave propagation phenomena in NPD media due to easy fabrication. Many applications of optical NPD structures have been proposed as well [19].

\subsection{Open structures and quasi-normal modes}

An open (leaky) optical structure (or more specifically an open resonator) can be seen as an inhomogeneity in a finite domain separated from the exterior by a partly open (transparent) boundary surface. Such an open structure loses energy to the exterior via radiation.

In multilayer structures resonances are manifested as a large transmission response of the system to the external excitation. More importantly, for specific finite multilayer structures, bandgaps can occur in the transmission response (here these are frequency ranges with very low transmission in contrast to the bandgaps of infinite periodic structure), and of the many resonances only those in the bandgaps (the defect modes) have high $\mathrm{Q}$ factors to be of practical interest. Then such a transmission resonance is associated with a purely real frequency. However, the notion of the resonance introduced in this way is somewhat obscure and hard to make precise in all cases of practical interest, see chapter 3 and 4.

As an alternative model for examining properties of multilayer structures an appropriate eigenvalue problem for the characteristic resonant frequencies (eigenvalues) and associated field profiles (eigenfunctions or modes) of open structures can be considered [20,21]. This approach is used in other branches of physics associated with wave scattering on finite structures [22, 23].

The simplest model of interest in optics, is a multilayer structure with $z$-dependent permittivity (refractive index) $\epsilon(z)=n^{2}(z)$ and constant permeability $\mu(z)=1$. This model describes an all-dielectric multilayer. Assuming a harmonic time dependence $E(z, t)=Q(z) e^{-j \omega t}$, the electric field for the TE-mode in the interior $x \in(L, R)$ is governed by the Helmholtz equation:

$$
\partial_{z}^{2} Q+\frac{\omega^{2}}{c^{2}} n^{2}(z) Q=0
$$

Viewing the finite multilayer as a passive, open optical structures with transparent boundaries which permit the leakage of energy to the exterior, outgoing wave boundary conditions

$$
\left(\partial_{z} Q+i \frac{\omega}{c} n_{i n} Q\right)_{z=L}=0 \text {, and }\left(\partial_{z} Q-i \frac{\omega}{c} n_{\text {out }} Q\right)_{z=R}=0 .
$$


are used. This constitutes an eigenvalue problem, further in the thesis refereed to as QNM problem, where the frequency $\omega$ is the complex eigenvalue and the field profile $Q(z)$ is the eigenfunction (Quasi-Normal Mode) [20, 24]. Note that this eigenvalue problem is nonlinear because the eigenvalue appears in the boundary conditions explicitly $[25,26]$.

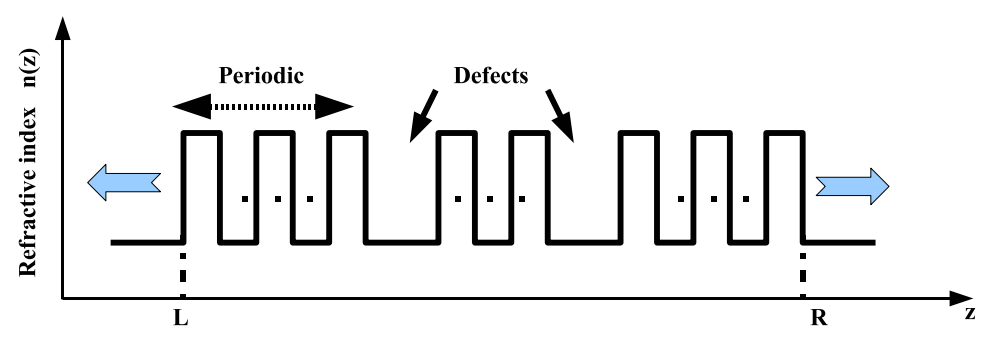

Figure 1.4: Open (leaky) optical multilayer structure with energy outflow to the exterior.

Commonly complex eigenfrequencies and QNMs for this type of structures can be found by solving appropriate transcendental equation for complex zeros. This transcendental equation can be obtained from the corresponding transfer matrix upon continuation in the complex plane [23]. Methods for solving this type of problem are numerous and there is a substantial literature devoted to this, a brief review follows in chapter 2 .

A more general method for solving the QNM eigen problem can be based on a suitable variational formulation. With a suitable discretization of the relevant functional, for instance by the Finite Element method, an algebraic nonlinear eigenvalue problem is obtained, see $[25,26]$ and references therein.

In finite structures, without dissipative losses due to absorption in the material, the main difference between open and closed optical resonators is that the resonant frequencies of closed resonators are real, while those of open resonators are complex [22, 20, 21]. In formal mathematical language, this difference arises because instead of Dirichlet or Neumann boundary conditions for the closed resonator, a radiation condition, allowing only outgoing waves, has to be imposed. Eigenfrequencies appear as discrete infinitely countable set of complex numbers $[22,20,21]$. However, QNMs (eigenfunctions) are unbounded for $x \rightarrow \pm \infty$, so they can not be normalized in the usual fashion over the whole spatial domain.

Open system do not satisfy energy conservation and the corresponding operators are no longer Hermitian. In general, eigenfunctions of non-Hermitian operators do not necessarily belong to a complete orthogonal basis, but rather form a set of non-orthogonal functions which may or may not be complete [27, 28, 29]. Decomposing a field on this set, even in the case of some form of completeness is not straightforward, and the usual tools involving field decomposition cannot be used [12].

Subject of our investigation are resonance phenomena in one- dimensional op- 
tical microcavities that are realized as defects in periodic dielectric multilayers,i.e structures with piecewise constant refractive index distribution. Suitable boundary conditions on finite domain can be applied in such a way that the properties of the open system are preserved [26].

When the time dependent problem of the energy leakage from such an open structure is considered, QNMs specify the field patterns in which the leaky optical structure would oscillate naturally after an initial excitation is withdrawn, thus representing damped oscillatory solutions of the wave equation. Then, QNMs and associated complex eigenvalues can be viewed as a proper means for solving the problem of energy leaking out of open structures, see [24] and references therein. Some results concerning this problem are reviewed in chapter 2.

The main aim of the approach described in chapters 2, 3 and 4 is to show that, by knowing a set of complex eigenfrequencies and associated QNMs for a given structure, we can reconstruct the frequency response of the structure to arbitrary excitation and/ or arbitrary perturbations of some parameters of the structure. Particularly the field representation is of major interest. The open, leaky nature of the optical system is directly incorporated.

\subsection{Negative index metamaterials}

Negative (refractive) index metamaterials are artificial composites, characterized by subwavelength features and a negative real part of the refractive index $[30,31]$. The negative real part of the refractive index arises in a frequency range where the real parts of both permittivity and permeability are negative [32, 30, 31]. Metamaterials are usually made of ordered or random arrangement of elementary "particles" that furnish designed effective electromagnetic response functions [33]. An important feature is that these elementary electric and magnetic "particles" are of subwavelength dimensions with respect to the target wavelength range. Then an incident wave does not resolve these subwavelength features of the metamaterial but rather "sees" the effective medium properties arising from the collective interaction of building blocks $[34,35]$. In this way, The Maxwell equations are complemented with the appropriate macroscopic constitutive relations incorporating the homogenized "effective" response functions for both electric and magnetic properties [36]. A striking consequence of the negative index metamaterials is that many of the basic laws of electromagnetism are reversed from those in ordinary media: reversal of the phase velocity, negative refraction, reversed Dopler effect, etc [32, 30, 31].

Negative index metamaterials seen as spatially homogeneous samples dictate that the phase velocity of an optical wave is in the opposite direction to the direction of the energy flow, i.e. Poynting vector, giving rise to the name backward-wave media or backward-phase velocity media. Also electric, magnetic field and propagation wave vector form the left-handed system which consequently leads to the name left-handed media. Although the terminology is not standard, the name that encompasses the fundamental property and is mostly used in the latest literature 
is Negative Refractive Index Metamaterials or Negative Index Metamaterial; we choose one of these terms further in the thesis.

The physics, the basic operating principles, and many applications of NIM are already proven or made available in the microwave range, see $[37,33,38]$ and references therein. Following remarkable results in the microwave range extended effort has been directed toward the realization of negative index metamaterials operating in the optical frequency range $[39,40,41]$. As initial results are very promising, it can be expected that technological advances might eventually enable efficient low loss NIMs for application in optics.

The possibilities offered by periodic all-dielectric or metal-dielectric photonic bandgap media might be greatly expanded by the introduction of electromagnetic metamaterials with negative index. Apart from many proposed applications and phenomena associated with subdiffraction imaging, see [31] and references therein, multilayers consisting of alternating dielectric (positive index material or PIM) and NIM layers offer new possibilities for the Photonic Bandgap Engineering not attainable by structures incorporating ordinary materials. Some of these new properties are a widening of the bandgap and flattening of the spectral transmission and reflection from finite structures [42, 43, 44], while at the same time the angular dependence of the transmission spectra in NIM-containing multilayers seem to be much weaker. Also, extended photonic bandgap engineering with NIM might give rise to omnidirectional bandgaps [44] and the so-called zero-n bandgap which appears when alternating PIM- NIM layers are stacked in such a way that the averaged refractive index is equal to zero [45, 46]. Some results suggest that these properties exist in periodic [46], quasi-periodic [47] and aperiodic structures [48].

Our interest in resonances of the multilayer structures is partially directed toward understanding the spectral transmission properties in multilayer structures containing NIM. In this respect, we address in chapter 5 and 6 the transmission spectra of periodic and non-periodic multilayers composed from positive and negative index metamaterials.

\subsection{Thermal radiation and multilayer structures}

The electromagnetic radiation emitted from the material bodies and originating from heating processes inside the material is called thermal radiation $[49,50]$. It represents the physical process associated with the microscopic processes of electromagnetic radiation emission induced by electron transitions in atoms, phonon transitions associated with molecular rotational and vibration modes and crystal lattice oscillations. In terms of wavelengths, it covers the ultraviolet spectrum, the visible light spectrum and the infrared spectrum [51,11].

The physical nature of processes associated with the thermal radiation can be described only by complementary pictures taking into account both quantum and classical physics $[3,51,11]$. However, in our considerations quantum processes associated with interaction of radiation and matter are handled implicitly. Because 
we are interested in phenomena associated with electromagnetic waves representing thermal radiation, we treat them classically: with macroscopic Maxwell equations and macroscopic material response functions permittivity, permeability and refractive index.

One of the topics of interest in optics is tailoring emittance/absorptance by changing the distribution of electromagnetic modes [13]. The theoretical foundation of the modification of thermal radiation by the photonic bandgap materials has been outlined in [52]. Thermal radiation is suppressed at frequencies inside the PBG, and enhanced at the frequencies of transmission resonances. In this way a spectral redistribution of thermal power is achieved. This can be interpreted in terms of a modification of the photonic density of modes within the photonic bandgap material and thus altering the thermal radiation spectrum.

On the practical side, the design of thermal sources with their emittance enhanced in a narrow solid angle has been of interest in the last few years [53, 54, $55,56]$. Selectivity in both frequency and directionality of these systems might be seen as effective antenna like behavior; a design goal dictated by expected applications in thermo-photovoltaic systems, infrared imaging systems, etc. Usually these systems are implemented with all-dielectric or metal-dielectric multilayer coatings on top of an absorbing substrate to enhance or suppress thermal emission from the substrate. This configuration enables thermal radiation control via the multilayer coatings applied as spectral and angular filters. This is readily implemented by the available thin-film technologies and it has been proved practically feasible to obtain antenna-like behavior for thermal sources in the IR range.

The computational approach used in this thesis relies on the Kirchhoff law for thermal radiation and the transfer matrix method. Kirchhoff law establishes an equality between absorptance and emittance for all frequencies, polarizations and propagation directions for an absorbing material object in thermal equilibrium $[49,50,52]$. This task is less complex than the direct computation of emission processes but still gives correct result in most of the cases of interest.

Advances in the technology of nanostructured materials may lead to the fabrication of materials with optical properties not readily found in nature, e.g. of NIMs for the optical range, see [13] and references therein. This offers new possibilities for the device design required for thermal radiation control. Further in this thesis, we investigate passive NIM-containing multilayers applied to tailor the spectral and angular emittance/absorptance distributions of an emitting substrate, see chapters 7 and 8 .

\subsection{Outline of the thesis}

In this thesis we are interested in resonance phenomena in optical multilayer structures. First, we direct our attention to the development of means for modeling multilayer structures as open systems. We adopt a quasi-normal mode description for both field profiles and transmission/ reflection responses. Specifically, we 
are interested in the field representation and in perturbation techniques for defect resonances of defect based one-dimensional photonic crystal.

In chapter 2, we introduce the fundamental notion of a resonance in a simple Fabry-Perot resonator, seen as closed system with hard boundaries, and also as an open system under external excitation and as a QNM problem. Then, the method for solving QNM problems for general multilayer structures is addressed. A recently developed method of a QNM expansion for solution of the transmission problem is briefly reviewed and applied to model examples of the optical defect microcavities in periodic multilayers. This method has its foundation in the specific pseudo-inner product introduced for projecting fields onto the QNM basis and in the specific completeness property for QNMs. Finally, time-independent QNM perturbation theory is considered. The existing theory from literature is briefly addressed along with a novel variational QNM perturbation theory.

In chapter 3, we specialize to resonances inside the bandgap of periodic multilayer mirrors that enclose the defect cavities. We investigate field approximations and characterization of the spectral transmission using variational principle and field template with only the most relevant QNMs accompanied by a specific mirror field. The method is applied to symmetric and non-symmetric structures with single and multiple defects.

Following the successful application of the variational principle for the field representation of defect resonances, chapter 4 deals with coupled optical defect cavities realized in finite one-dimensional Photonic Crystals. Here, single defect structures (photonic crystal atoms) can be viewed as elementary building blocks for multiple-defect structures (photonic crystal molecules) with more complex functionality. The QNM description links the resonant behavior of individual PC atoms to the properties of the PC molecules via eigenfrequency splitting. A variational principle for QNMs permits to predict the QNMs and the complex eigenfrequencies in PC molecules starting with a field template incorporating the relevant QNMs of the PC atoms. Further, both the field representation and the resonant spectral transmission close to these resonances are obtained from a variational formulation of the transmission problem using a template with the most relevant QNMs. The method is applied to both symmetric and nonsymmetric single and multiple cavity structures with weak or strong coupling between the defects.

A second class of problems that we address concerns multilayer structures incorporating negative index metamaterials. The Transfer Matrix Method, as outlined in chapter 1 , is technique applied for this purpose.

Chapter 5 starts with a brief review of some basic properties of the negative index metamaterials. Then, we address some novel properties of the bandgap structure and transmission spectra obtained by the introduction of NIM in the construction of the infinite and the finite multilayers. A second part of chapter 5 reviews some basics concerning thermal radiation. Specifically Planck's and Kirchhoff's law are addressed. Finally, we introduce the basic concept of thermal radiation antenna, i.e. a system that enables both spectral and directional selectivity of the thermal power spectrum emitted by some material object. 
Chapter 6 deals with the optical transmission spectra of aperiodic Thue-Morse multilayers composed from alternating layers of media with positive and negative refractive index. We investigate transmission resonances and the field distributions associated with them for finite structures. The angular dependence of the transmission spectra and the robustness of the transmission resonances with respect to the phase shift modulation are investigated. Non-dispersive and lossless, as well as realistic dispersive and lossy materials are considered.

The design of multilayer coatings applied to enable spectral and directional control over thermal radiation from emitting substrates has been of interest in the last years. In chapter 7 we investigate modification of the thermal radiation power spectrum in periodically ordered multilayers containing negative index metamaterials. Both on-axis and off-axis radiation are analyzed.

An additional degree of freedom in the design of thermal radiation antennas may be expected when more general multilayer designs are used. In chapter 8 we investigate wave propagation through one-dimensional stacks of alternating positive and negative refractive index layers arranged as truncated (pre-fractal) Cantor multilayers. Dispersion and absorptive losses for both on-axis and off-axis radiation are taken into account.

Brief remarks on possible directions for future research concerning the topics discussed in chapters 2-8, conclude this thesis. 


\title{
Chapter 2
}

\section{Resonances and quasi-normal modes}

\begin{abstract}
Subject of our investigation are resonance phenomena in optical cavities realized as defects in one-dimensional structures. Upon viewing the cavity as a passive open system with intrinsically leaky behavior due to the open boundaries where waves are permitted to leave the structure, the cavity can be characterized in terms of complex eigenfrequencies and quasi-normal modes (eigenfunctions). Our aim is to predict the response of the structure to the external excitation and/or internal perturbations, solely based on the knowledge of eigenfrequencies of the QNMS supported by the structure. A specific two-component formalism and a related QNM expansion method is briefly reviewed and applied to model examples of the optical defect microcavities in periodic multilayers. Also, a time-independent QNM perturbation theory is considered.
\end{abstract}


Specific subject of our investigation are resonance phenomena in optical cavities realized as defects in multilayer structures. Resonance phenomena are usually associated with a large response of an system to some external excitation. The response is determined to a large extent by intrinsic properties of the system regardless of the excitation. One of the features of all realistic optical structures is that they are open, non-conservative systems. Apart from possible material absorption losses, radiation may escape from the system carrying energy to the exterior through open boundaries.

For simplicity consider an optical structure with a piecewise constant refractive index distribution $n(x)$ within the finite domain $x \in(0, L)$ and an exterior with constant refractive index $n_{0}$. The nature of the boundaries is such that they permit leakage of the energy to the outside, i.e. the structure is said to be open (leaky) [20].

A first model of interest is an optical structure without external excitation with only the outgoing waves in the exterior. The wave propagation is described by the scalar wave equation for the electric field

$$
\frac{\partial^{2} E(z, t)}{\partial x^{2}}-\frac{n^{2}(x)}{c^{2}} \frac{\partial^{2} E(x, t)}{\partial t^{2}}=0
$$

with associated outgoing wave boundary conditions

$$
\left(\frac{\partial E}{\partial x}-\frac{n_{0}}{c} \frac{\partial E}{\partial t}\right)_{x=0}=0, \quad\left(\frac{\partial E}{\partial x}+\frac{n_{0}}{c} \frac{\partial E}{\partial t}\right)_{x=L}=0
$$

where $c$ is speed of light in vacuum and exterior refractive index $\left.n\right|_{x=0}=\left.n\right|_{x=L}=$ $n_{0}$. These boundary conditions can be simply checked by splitting the general solution of the wave equation in the homogeneous medium in forward and backward traveling waves with respect to the orientation of coordinate axis [1]. Such a simple form of the boundary conditions (2.2) requires that the exterior is homogeneous. If a harmonic time dependence for the electric field $E(x, t)=Q(x) e^{-i \omega t}$ is assumed, then (2.1) becomes the Helmholtz equation

$$
\frac{\partial^{2} Q(x)}{\partial x^{2}}+\frac{n^{2}(x)}{c^{2}} \omega^{2} Q(x)=0
$$

with outgoing wave boundary conditions

$$
\left(\frac{\partial Q}{\partial x}+i \omega \frac{n_{0}}{c} Q\right)_{x=0}=0, \quad\left(\frac{\partial Q}{\partial x}-i \omega \frac{n_{0}}{c} Q\right)_{x=L}=0 .
$$

Equation (2.3) together with (2.4) represents an eigenvalue problem for the complex frequency as eigenvalue and associated Quasi-Normal Mode as eigenfunction. The eigenvalue problem is nonlinear because the eigenfrequency appears in the boundary conditions explicitly. We are interested in nontrivial solutions with negative imaginary part $\operatorname{Im}\left(\omega_{k}\right)<0$ of the eigenfrequency. When considered in the time domain these fields are damped oscillating solutions, where the damping 
is controlled by $\operatorname{Im}\left(\omega_{k}\right)<0$. The imaginary part of the frequency is related to the energy decay and closely related to the Q-factor of the cavity, see $[22,26]$ and references therein. The eigenfunctions are unbounded on the real line blowingup at spatial infinity. Solutions appear always in pairs $\left(\omega_{k}, Q_{k}\right)$ and $\left(-\omega_{k}^{*}, Q_{k}^{*}\right)$ $[20,57]$. The QNMs are in fact the natural modes of the optical structure that represent damped oscillations of the field after an initial excitation is withdrawn, see $[22,58]$ and references therein.

Note that the present $1 \mathrm{D}$ eigenvalue problem involves local boundary conditions. However, in higher dimensions this is not possible and the radiation condition permitting only outgoing waves can be approximated only in the form of nonlocal boundary conditions using Dirichlet-to-Neumann maps [26, 59]. Common computational approaches then involve local approximations, e.g. by means of perfectly matched layers [22,60].

As a second model we consider the structure under an external excitation by an incoming wave. The wave equation (2.1) is accompanied by a transparent influx boundary condition at the side $x=0$ of the structure where a given incidennce wave impinges:

$$
\left(\frac{\partial E}{\partial x}-\frac{n_{0}}{c} \frac{\partial E}{\partial t}\right)_{x=0}=b(t), \quad\left(\frac{\partial E}{\partial x}+\frac{n_{0}}{c} \frac{\partial E}{\partial t}\right)_{x=L}=0
$$

where

$$
b(t)=2\left(\frac{\partial E_{i n}}{\partial x}\right)_{x=0}=-2 \frac{n_{0}}{c}\left(\frac{\partial E_{i n}}{\partial t}\right)_{x=0} .
$$

represents the incoming wave. This boundary condition is obtained by noting that the field at the boundary $x=0$ can be decomposed as the sum $E=E_{s}+E_{i n}$, where $E_{s}$ is the scattered wave component satisfying outgoing wave b.c.'s and $E_{i n}$ is the given incoming wave. Then taking the derivative with respect to the spatial variable and the time variable at the position of the boundary $x=0$ and eliminating $E_{s}$ the inhomogeneous boundary condition follows.

For harmonic time dependence the same Helmholtz equation (2.3) is obtained, now with inhomogeneous boundary conditions

$$
\left(\frac{\partial E}{\partial x}+i \omega \frac{n_{0}}{c} E\right)_{x=0}=b(\omega), \quad\left(\frac{\partial E}{\partial x}-i \omega \frac{n_{0}}{c} E\right)_{x=L}=0 .
$$

For a harmonic incident wave of the form $E_{i n}=A_{i n c} \exp \left(\frac{\omega n_{0}}{c} x-\omega t\right)$ the inhomogeneity is $b(\omega)=2 i \omega \frac{n_{0}}{c} A_{\text {inc }}$ with given real frequency $\omega \in \mathbb{R}$ and given input amplitude $A_{\text {inc }}$ given. This is the transmission problem as introduces in chapter 1 .

Our aim is to predict the response of the structure to external excitation and/or parameter perturbations, solely based on the knowledge of eigenfields and eigenfrequencies of the QNMs supported by the cavity. 


\subsection{Quasi-normal modes and multilayers}

The QNM problem for a multilayer structure with a homogeneous exterior can be solved by means of adaptation of the transfer matrix method outlined in section (1.2.2). If only outgoing waves are allowed in the exterior, the incoming wave amplitude $A_{0}$ is set to zero. Then the equation for the overall transfer matrix (1.56) becomes

$$
\left(\begin{array}{c}
0 \\
A_{L}
\end{array}\right)=\left(\begin{array}{ll}
m_{11} & m_{12} \\
m_{21} & m_{22}
\end{array}\right)\left(\begin{array}{c}
A_{R} \\
0
\end{array}\right)
$$

where $A_{R}$ and $A_{L}$ are the amplitudes of the left and right travelling outgoing waves. Equation (2.8) can be satisfied with nontrivial $A_{L}, A_{R}$ if

$$
m_{11}(\omega)=0, \text { for } \omega \in \mathbb{C},
$$

i.e. solutions are found by analytic continuation of (1.56) into the complex plane [23]. In principle one would have to expect infinitely many discrete solutions with different algebraic multiplicity, but in the case with homogeneous exterior these solutions are in fact simple zeros [22]. Note, that this description has an equivalent form that connects the incoming to the outgoing waves via so called scattering matrices [10]. Then complex eigenfrequencies may be interpreted as complex poles of the scattering matrix [6]. Alternatively, they are poles of the reflection and the transmission transfer functions obtained by the multiple scattering method [12]. In fact, this is a standard interpretation of the complex eigenfrequencies [6]. To actually find complex solutions of (2.9) we use a standard Newton type method [61].

\subsubsection{Resonances and QNMs of single dielectric slab}

If a closed resonator model is considered, fields are identical to zero at the boundaries [2], and if there are no internal losses due to material absorption, such system allows storing of electromagnetic energy forever. Mathematical model of this system is an eigenvalue problem of the Sturm-Liouville type [62, 63, 64]. Eigenfrequencies are real and the eigenfunctions form a complete orthonormal set $[62,2,12]$. Then, an arbitrary field distributions inside the cavity can be decomposed into these eigenfunctions (normal modes), while resonances are identified with the corresponding real eigenfrequencies and the normal modes of the system are standing waves with nodal points at the boundaries $[62,2,12]$.

When the optical resonator is open, i.e. the boundaries of the cavity allow energy leakage into the exterior situation becomes more complicated. As an example we look at a simple 1D Fabry-Perot type resonator structure with two semitransparent mirrors [4]; in our setting this can be a slab of dielectric material (refractive index $n_{S}$ and thickness $L_{S}$ ) separated from the vacuum environment (refractive index $n_{0}$ ).

We consider the externally driven system, when waves are incident onto the structure and can be either reflected or transmitted. This is a transmission problem, 
such that we seek for solutions both in the exterior and interior with specified input amplitude and real frequency of the incoming wave. Here a resonance is usually understood as a frequency where the transmission coefficient attains maximum a value. According to a transfer matrix solution (1.2.2), it is easy to show after some algebra that the transmittance can be written as

$$
T(\omega)=\frac{\left(1-r^{2}\right)^{2}}{1+r^{4}-2 r^{2} \cos \left(2 \frac{\omega}{c} n_{s} L_{s}\right)}
$$

where $r=\frac{n_{s}-n_{0}}{n_{s}+n_{0}}$ is the interface amplitude reflection coefficient. The transmission resonances $T\left(\omega_{t r}\right)=1$ occur when the frequency attains values with

$$
\cos \left(2 \frac{\omega}{c} n_{s} L_{s}\right)=1, \text { or } \omega_{t r}=p \frac{\pi c}{n_{s} L_{s}}, \text { for } p= \pm 1, \pm 2, \pm 3, \ldots
$$

In fact this can be interpreted as a condition for constructive interference, i.e. round-trip of the wave in the resonator is an integer multiple of the wavelength $[1,4]$.

If we require only outgoing waves in the exterior, then due to the same simple transfer matrix representation the system of equations can be satisfied only for complex frequencies

$$
\omega_{q}=p \frac{\pi c}{n_{s} L_{s}}-i \frac{c}{n_{s} L_{s}} \ln (1 / r) \text { for } p= \pm 1, \pm 2, \pm 3, \ldots .
$$

Note that the same result can be obtained if one find complex poles of (2.10).

When the thickness of the slab is set to be quarter-wavelength $L_{s}=\frac{\lambda_{0}}{2 n_{s}}$ for target wavelength $\lambda_{0}=2 \pi c / \omega_{0}$, the transmission resonance frequencies and eigenfrequencies reads

$$
\omega_{t r}=p\left(2 \omega_{0}\right) \text { and } \omega_{q}=p\left(2 \omega_{0}\right)-i \frac{2 \omega_{0}}{\pi} \ln (1 / r) .
$$

Note that the transmission resonance frequencies and real parts of the complex eigenfrequencies are identical. Therefore, incident wave is perfectly transmitted if the frequency of the incoming wave is identical to the real part of a complex eigenfrequency. However, if a multilayer is considered, the real parts of the eigenfrequencies and the transmission resonance frequencies are not equal in general, although they may be very close [65]. We may expect in more complicated structures that a resonant transmission occurs when the frequency of the incident wave is close to the real part of a complex eigenfrequency.

\subsubsection{QNMs and defect resonances in multilayers}

As an example we compare a periodic and a defect structure coded as $(H L)^{8} H$ and $(H L)^{4} 2 H(L H)^{4}$ respectively. Here $H$ and $L$ denote high and low index layers with refractive indices $n_{H}=3.42$ and $n_{L}=1.45$. The thicknesses are chosen as quarter-wavelength with respect to the target wavelength $\lambda_{0}=2 \pi c / \omega_{0}=$ 

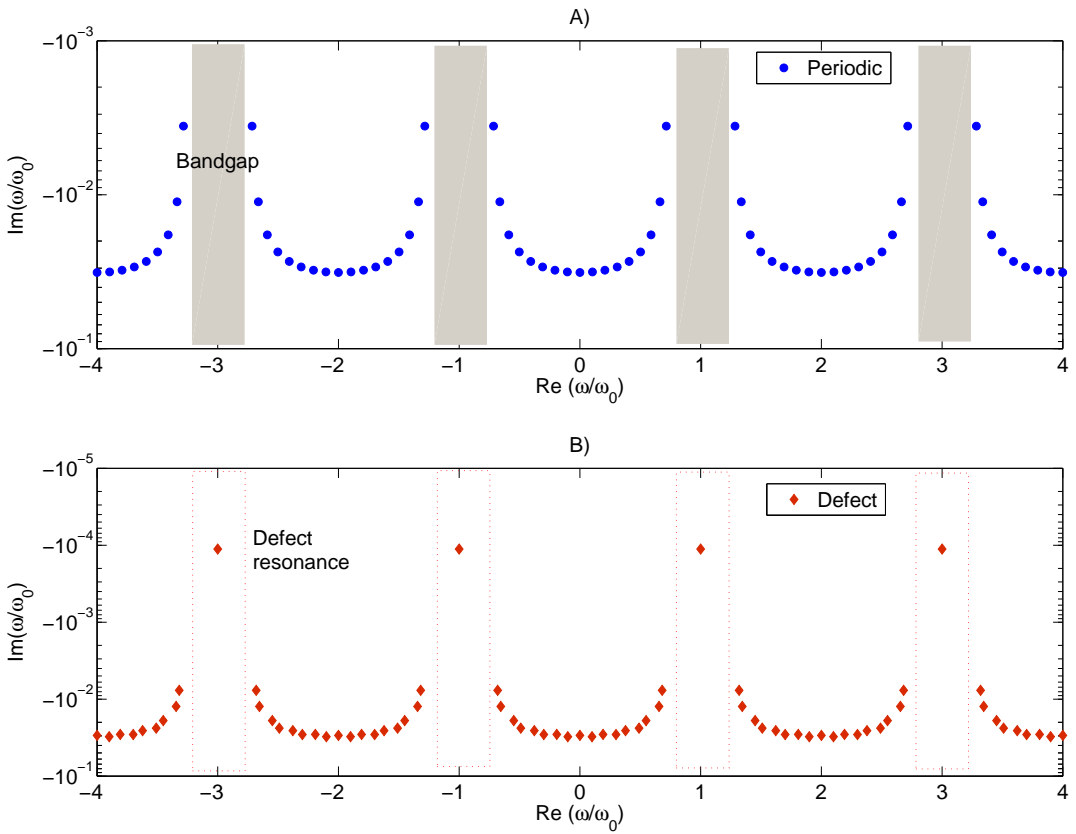

Figure 2.1: Complex eigenfrequencies for periodic multilayer A) and defect multilayer $B)$.

$1.55 \mu \mathrm{m}$. Defect structure has a central layer of half-wavelength thickness. All materials are assumed to be nonmagnetic.

Solutions of equation (2.9) are the complex eigenfrequencies depicted in Figure 2.1. For the periodic multilayer they appear to be arranged throughout complex plane in a specific pattern with distinctive strips being without eigenfrequencies as shown in Figure 2.1 A). These regions (gray patches) seem to be a reminiscence of the bandgap structure associated with the infinite periodic structure. Indeed, the edge frequencies of the band-stop frequency range for the finite structure under external excitation (not shown here) are close to the real parts of edge eigenfrequencies, i.e those closest to the strips in Figure $2.1 \mathrm{~A}$ ). The infinite countable set of e eigenfrequencies may be partitioned into the sets of eigenfrequencies having the same imaginary, with their real parts being integer multiple of $2 \omega_{0}$. Similar observations also have been made in [65]. For the defect structure eigenfrequencies are present inside the "bandgap" region as can be seen in Figure 2.1 B). This is expected from the known property that in the spectral transmission of this structure a transmission resonance appears inside the bandgaps $[4,8,66]$.

Owing to the similar structure and the arrangement of the complex eigenfrequencies in the complex plane for the periodic and defect structures, same relation between these two situations might be expected. Let us start with the structure $(H L)^{4} \chi H(L H)^{4}$, where $\chi \in(1,2)$, meaning that for $\chi=1$ structure is peri- 

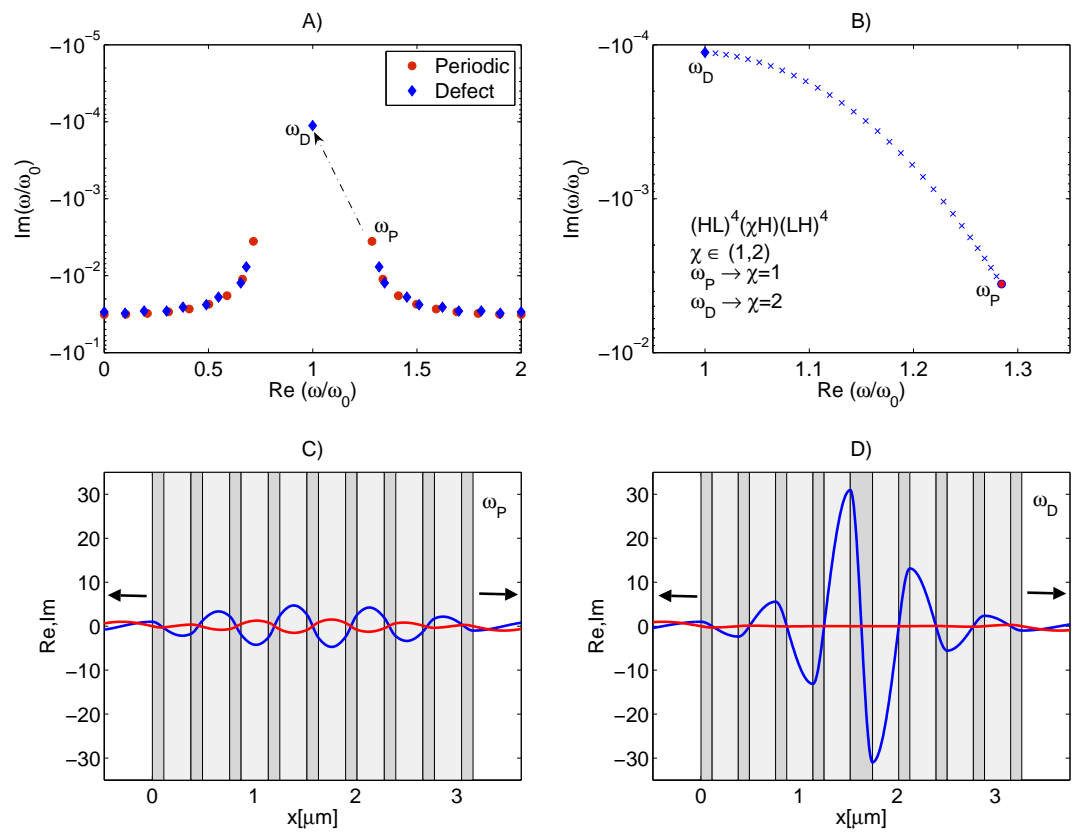

Figure 2.2: A) Eigenfrequencies for the periodic and defect multilayer B) Shift of the band edge eigenfrequency with a quasi-continuous increase of the width of the central layer C) QNMs for band edge eigenfrequency (periodic multilayer) and D) defect eigenfrequency (defect multilayer) .

odic and for $\chi=2$ it becomes a defect structure. Corresponding eigenfrequencies inside the first band are depicted in Figure 2.2 A). By changing the parameter $\chi$ in a given range and by computing eigenfrequencies in each step we are able to track the eigenfrequencies in the complex plane. We observe that the bandedge eigenfrequency $\omega_{P}$ shifts inside the bandgap and in fact turns into the defect eigenfrequency $\omega_{D}$ with very small imaginary part and with it's real part in the middle of the bandgap, as shown in Figure 2.2 B).

Corresponding QNMs for these two extremal cases are shown in Figure 2.2 C) and D) for eigenfrequencies $\omega_{P}$ and $\omega_{D}$ respectively. We observe a drastic change of the QNMs profile. Whereas the QNM for $\omega_{P}$ appears to be almost equally distributed throughout the whole structure, the one associated with $\omega_{D}$ is localized in the vicinity of the defect layer, with noticeable amplitude enhancement. These properties are similar to those of field profiles associated with transmission and defect resonances [4] as well as those of pass-band states and defect states, i.e. extended and localized states in the infinite structure [8].

The eigenvalue problem for an infinite structure with periodic boundary conditions leads to a spectrum with in general both discrete and continuous parts, characterizing structure in terms of bandgaps [8]. The pattern observed above in 
the QNM spectrum of the present finite structure, suggests an an analogues. Some results concerning the aymptotics of the QNMs spectrum if a structure is infinitely extended were reported for 1D problems dealing with scattering resonances and bandgaps of finite and infinite periodic potentials in quantum mechanics, see [67] and references therein.

\subsection{Two-component formalism and QNM expansion}

The full set of complex eigenfrequencies and QNMs supported by an arbitrary multilayer structure on the one hand, and the spectral transmission and the field profiles for the structure under external excitation on the other hand may be seen as two ways to describe the properties of the optical system. Consequently, using QNMs and the corresponding eigenfrequencies as a means for the solution of the transmission problem seems to be a reasonable way to connect these two descriptions. For this task a certain QNM expansion method that has been proposed recently [24] may be used. The method is based on the theory outlined in [68] and the two-component formalism described in [69], [70]. This method has been adapted recently for $1 \mathrm{D}$ photonic crystal structures, see $[65,71,72]$ and references therein.

We review some results concerning specifically approximations of the spectral transmission and the associated fields in the transmission problem. Examples for a single defect 1D photonic bandgap structure with comments on some silent features of the outlined method are given below.

Time domain The problem described by the second order wave equation (2.1) can be expressed in terms of two first order in time equations, provided that two components are introduced: the field variable $E(x, t)$ and a component often called conjugate variable, defined as $\widehat{E}(x, t)=\frac{n^{2}(x)}{c^{2}} \partial_{t} E(x, t)$. The full description is then given via the vector $\mathbf{E}=[E \widehat{E}]^{T}$. If we introduce an operator $\mathcal{H}$ defined as

$$
\mathcal{H}=\left(\begin{array}{cc}
0 & \frac{c^{2}}{n^{2}(x)} \\
\partial_{x}^{2} & 0
\end{array}\right),
$$

then the time evolution equation reads

$$
\partial_{t} \mathbf{E}=\mathcal{H} \mathbf{E}
$$

and is accompanied by outgoing wave boundary conditions

$$
\left(\frac{\partial E}{\partial x}-\frac{c}{n_{0}} \widehat{E}\right)_{x=0}=0, \quad\left(\frac{\partial E}{\partial x}+\frac{c}{n_{0}} \widehat{E}\right)_{x=L}=0 .
$$

The time evolution equation in the two-component form (2.15) with (2.16) is equivalent to the second order wave equation [73]. 
Frequency domain For harmonic time dependence in both components $\mathbf{E}(x, t)=$ $\mathbf{Q}(x) e^{-i \omega t}$, the QNM problem reads

$$
\mathcal{H} \mathbf{Q}(x)=(-i \omega) \mathbf{Q}(x)
$$

with boundary conditions

$$
\left(\frac{\partial Q}{\partial x}-\frac{c}{n_{0}} \widehat{Q}\right)_{x=0}=0, \quad\left(\frac{\partial Q}{\partial x}+\frac{c}{n_{0}} \widehat{Q}\right)_{x=L}=0 .
$$

Note that this eigenvalue problem is linear because the eigenvalue is not explicitly present in the boundary conditions. Two component eigenfunction is given as $\mathbf{Q}(x)=\left[\left(Q(x)-i \omega \frac{n^{2}(x)}{c^{2}} Q(x)\right]^{T}\right.$ where $Q$ is the same as the solution of the problem (2.3), (2.7).

\subsubsection{Pseudo-inner product, orthogonality and completeness}

Let us introduce bilinear map for the two-component functions with the following form

$\left\langle\mathbf{E}^{(1)}, \mathbf{E}^{(2)}\right\rangle=\int_{0}^{L}\left(E^{(1)} \widehat{E}^{(2)}+E^{(2)} \widehat{E}^{(1)}\right) d x+\frac{n_{0}}{c}\left(\left.E^{(1)} E^{(2)}\right|_{x=0}+\left.E^{(1)} E^{(2)}\right|_{x=L}\right)$.

The time-evolution operator $\mathcal{H}$ is symmetric under this bilinear map within the space of functions satisfying outgoing wave boundary conditions. The symmetry subsequently leads to the orthogonality of the QNMs and establishes a projection technique for QNM expansions suitable for open optical structures, see [24] and references therein.

Note that this bilinear map involves two components, i.e. both field and the time-derivative of the field. ${ }^{1}$ Also, the bilinear map explicitly incorporate boundary data. Therefore in the frequency domain bilinear map may be difficult to apply for arbitrary fields. This is one of the reasons to use the formalism first in the time-domain and then apply a Fourier transform to obtain the frequency domain description. Note that (2.19) is not positive definite.

Although this seems to be a complication if (2.19) is used with arbitrary functions, benefit is that due to the symmetry of the time evolution operator and the orthogonality of the QNMs the bilinear map may be used for an effective projection onto QNM basis. Therefore it may be seen as pseudo-inner product [27, 28]. Some relations of this bilinear map with the usual inner product have been considered in $[74,75]$.

Symmetry With the bilinear map (2.19) the operator (2.14) is symmetric in the space of functions satisfying the outgoing wave boundary conditions

$$
\left\langle\mathbf{E}^{(1)}, \mathcal{H} \mathbf{E}^{(2)}\right\rangle=\left\langle\mathcal{H} \mathbf{E}^{(1)}, \mathbf{E}^{(2)}\right\rangle .
$$

\footnotetext{
${ }^{1}$ When considered in the frequency domain, for QNMs that satisfy (2.17), in a single component form it also involves the frequency explicitly in (2.19).
} 
To show this property, let us start from the left-hand side of (2.20)

$$
\left\langle\mathbf{E}^{(1)}, \mathcal{H} \mathbf{E}^{(2)}\right\rangle=\int_{0}^{L} d x\left(\left(E^{(1)} \partial_{x}^{2} E^{(2)}+\widehat{E}^{(1)} \frac{c^{2}}{n^{2}(x)} \widehat{E}^{(2)}\right)+S_{t}\right.
$$

where

$$
S_{t}=\frac{n_{0}}{c}\left(\left(E^{(1)} \frac{c^{2}}{n_{0}^{2}} \widehat{E}^{(2)}\right)_{x=0}+\left(E^{(1)} \frac{c^{2}}{n_{0}^{2}} \widehat{E}^{(2)}\right)_{x=L}\right) .
$$

Next, let us apply partial integration twice in the first integral (2.21) and use boundary conditions (2.16) to obtain

$$
\left\langle\mathbf{E}^{(1)}, \mathcal{H} \mathbf{E}^{(2)}\right\rangle=\int_{0}^{L}\left(E^{(2)} \partial_{x}^{2} E^{(1)}+\widehat{E}^{(2)} \frac{c^{2}}{n^{2}(x)} \widehat{E}^{(1)}\right) d x+S_{1}+S_{2}+S_{t} .
$$

where

$$
S_{1}=\left.\left(E^{(1)} \partial_{x} E^{(2)}\right)\right|_{0} ^{L}=-S_{t}
$$

and

$$
S_{2}=-\left.\left(E^{(2)} \partial_{x} E^{(1)}\right)\right|_{0} ^{L}=\frac{n_{0}}{c}\left(\left(E^{(2)} \frac{c^{2}}{n_{0}^{2}} \widehat{E}^{(1)}\right)_{x=0}+\left(E^{(2)} \frac{c^{2}}{n_{0}^{2}} \widehat{E}^{(1)}\right)_{x=L}\right)
$$

Finally, we get

$$
\left\langle\mathbf{E}^{(1)}, \mathcal{H} \mathbf{E}^{(2)}\right\rangle=\int_{0}^{L}\left(E^{(2)} \partial_{x}^{2} E^{(1)}+\widehat{E}^{(2)} \frac{c^{2}}{n^{2}(x)} \widehat{E}^{(1)}\right) d x+S_{2}
$$

which is equal to the right-hand side of (2.20) and proves the symmetry of $\mathcal{H}$ under action of the bilinear map.

QNM orthogonality From the symmetry of the operator $\mathcal{H}$ defined in (2.20) follows the orthogonality of the eigenfunctions (2.17). First, let us assume that there are two distinct solutions, $\left(-i \omega_{k}, \mathbf{Q}_{k}\right)$ satisfying the eigenvalue equation

$$
\mathcal{H} \mathbf{Q}_{k}=\left(-i \omega_{k}\right) \mathbf{Q}_{k}
$$

Let us project (2.27) onto QNMs $\mathbf{Q}_{m}$

$$
\left\langle\mathbf{Q}_{m}, \mathcal{H} \mathbf{Q}_{k}\right\rangle=\left\langle\mathbf{Q}_{m},\left(-i \omega_{k}\right) \mathbf{Q}_{k}\right\rangle .
$$

If we use the symmetry of $\mathcal{H}$ and the linearity of the bilinear map, we obtain

$$
\left\langle\mathcal{H} \mathbf{Q}_{m}, \mathbf{Q}_{k}\right\rangle=\left(-i \omega_{k}\right)\left\langle\mathbf{Q}_{m}, \mathbf{Q}_{k}\right\rangle,
$$

and

$$
\left(-i \omega_{m}\right)\left\langle\mathbf{Q}_{m}, \mathbf{Q}_{k}\right\rangle=\left(-i \omega_{k}\right)\left\langle\mathbf{Q}_{m}, \mathbf{Q}_{k}\right\rangle
$$

which gives

$$
\left(\omega_{m}-\omega_{k}\right)\left\langle\mathbf{Q}_{m}, \mathbf{Q}_{k}\right\rangle=0 .
$$


If we assume that $\omega_{k} \neq \omega_{m}$ for $k \neq m$ the relation above requires

$$
\left\langle\mathbf{Q}_{m}, \mathbf{Q}_{k}\right\rangle=N_{k} \delta_{m k},
$$

where $\delta_{m k}=1$ if $m=k$, and $\delta_{m k}=0$ if $m \neq k$; the normalization constant $N_{k}$ is a complex number.

For valid QNMs, solution of (2.17) $\mathbf{Q}_{k}=\left[Q_{k}-i \omega_{k} \frac{n^{2}}{c^{2}} Q_{k}\right]^{T}$ we have

$$
\left\langle\mathbf{Q}_{k}, \mathbf{Q}_{k}\right\rangle=2\left(-i \omega_{k}\right) \int_{0}^{L}\left(\frac{n^{2}(x)}{c^{2}} Q_{k}^{2} d x\right)+\frac{n_{0}}{c}\left(\left(Q_{k}^{2}\right)_{x=0}+\left(Q_{k}^{2}\right)_{x=L}\right) .
$$

Thus, the bilinear map (2.19) provides orthogonality relations of eigenfunctions in the two-component formalism. We will exploit this property in the following sections. This relation can be obtained directly from the Helmholtz equation by simply multiplying (2.3) with appropriate QNMs, subsequent partial integration, and using the outgoing wave boundary conditions.

Note, that the norm associated with the QNMs via bilinear map is in general the complex number and thus can not be interpreted as usual positive definite norm [24].

Completeness of QNMs Let us introduce a simultaneous expansion of both components of an open cavity field $E(x, t)$ and $\widehat{E}(x, t)$ in terms of the components of the eigenfunctions $Q(x)$ and $\widehat{Q}(x)$ :

$$
\mathbf{E}(x, t)=\sum_{k=-\infty}^{\infty} a_{k}(t) \mathbf{Q}_{k}(x) .
$$

The same time dependent coefficient $a_{k}(t)$ is used for both components.

Given the orthogonality of the QNMs, the two component formalism suggests appears to provide effective projection technique for a QNM expansion based on the introduced bilinear map. However, the approximations may be better justified if some completeness property for the full set of QNMs can be established.

In $[69,68]$ the completeness of QNMs has been approached through a representation of the Green function satisfying causal initial conditions and asymptotic outgoing wave boundary conditions. The following relations are obtained:

$$
\sum_{k=-\infty}^{\infty} \frac{Q_{k}(x) Q_{k}(y)}{\left\langle\mathbf{Q}_{k}, \mathbf{Q}_{k}\right\rangle}=0, \text { for } x, y \in[0, L]
$$

and

$$
\sum_{k=-\infty}^{\infty} \frac{n^{2}(x)}{c^{2}} \frac{\omega_{k} Q_{k}(x) Q_{k}(y)}{\left\langle\mathbf{Q}_{k}, \mathbf{Q}_{k}\right\rangle}=\delta(x-y), \text { for } x, y \in[0, L] .
$$

These relations are interpreted as completeness relations under the specific conditions that exterior of the structure is homogeneous and (2.34) is valid only inside 
the domain of the structure [69]. The reasoning on completeness properties for QNMs in $[57,76,77]$ led to the observations that the set of the QNMs is in fact overcomplete. Then, uniqueness of the QNM expansion is not guaranteed any more.

\subsubsection{QNM expansion and exponentially decaying fields}

Let us represent an arbitrary field inside the cavity, that satisfies outgoing wave boundary conditions (2.16), in terms of quasi-normal modes (2.34), and project with (2.19) this distribution onto a QNM eigenfunction $\mathbf{Q}_{m}$ :

$$
\left\langle\mathbf{Q}_{m}, \mathbf{E}\right\rangle=\left\langle\mathbf{Q}_{m}, \sum_{k=-\infty}^{\infty} a_{k}(t) \mathbf{Q}_{k}\right\rangle .
$$

Next, orthogonality of the QNMs leads to

$$
\left\langle\mathbf{Q}_{k}, \mathbf{E}\right\rangle=a_{k}(t)\left\langle\mathbf{Q}_{k}, \mathbf{Q}_{k}\right\rangle,
$$

which gives a projection formula for the expansion coefficients

$$
a_{k}(t)=\frac{\left\langle\mathbf{Q}_{k}, \mathbf{E}\right\rangle}{\left\langle\mathbf{Q}_{k}, \mathbf{Q}_{k}\right\rangle} .
$$

To solve the problem of the time-evolution of an initial optical cavity field, subjected to outgoing wave boundary conditions, let us differentiate with respect to time the projection formula (2.39):

$$
\partial_{t} a_{k}(t)=\frac{\left\langle\mathbf{Q}_{k}, \partial_{t} \mathbf{E}\right\rangle}{\left\langle\mathbf{Q}_{k}, \mathbf{Q}_{k}\right\rangle} .
$$

Then from the time evolution equation (2.15) it follows that

$$
\partial_{t} a_{k}(t)=\frac{\left\langle\mathbf{Q}_{k}, \mathcal{H} \mathbf{E}\right\rangle}{\left\langle\mathbf{Q}_{k}, \mathbf{Q}_{k}\right\rangle} .
$$

The symmetry (2.20) of $\mathcal{H}$ and the eigenvalue equation (2.17) lead to

$$
\partial_{t} a_{k}(t)=\frac{\left(-i \omega_{k}\right)\left\langle\mathbf{Q}_{k}, \mathbf{E}\right\rangle}{\left\langle\mathbf{Q}_{k}, \mathbf{Q}_{k}\right\rangle}
$$

According to (2.38) the expansion coefficients satisfy the equation

$$
\partial_{t} a_{k}(t)+i \omega_{k} a_{k}(t)=0 .
$$

This equation describes the time evolution of an arbitrary field inside the cavity satisfying outgoing wave boundary conditions in terms of the complex frequencies corresponding phase factors in the expansion

$$
\mathbf{E}(x, t)=\sum_{k=-\infty}^{\infty} a_{k}(0) \mathbf{Q}_{k}(x) e^{-i \omega_{k} t}, \text { for } t>0,
$$


where

$$
a_{k}(0)=\frac{\left\langle\mathbf{Q}_{k}, \mathbf{E}(x, 0)\right\rangle}{\left\langle\mathbf{Q}_{k}, \mathbf{Q}_{k}\right\rangle} .
$$

The initial conditions necessary to be specified in this case are both field components at the time $t=0$, that is $E(x, 0)$ and $\widehat{E}(x, 0)=\frac{n^{2}(x)}{c^{2}} \partial_{t} E(x, 0)$.

The representation of time-exponentially decaying states via similar QNM superposition has been considered in [78]. In particular, it has been shown that decaying state can be represented by a QNM superposition for some smooth initial conditions in a localized spatial region, see [78] and references therein.

\subsubsection{QNM expansion and the transmission problems}

In the transmission problem, multilayer structures interact with the environment through external excitation by an incoming wave. We are seeking a description of the transmission problem via a QNM expansion method [73], [65],[71],[72].

Time domain The total field of the optical structure subjected to the influx from one side is described, within the two-component formalism, by means of the time evolution equation (2.15) and the transparent influx boundary conditions

$$
\left(\frac{\partial E}{\partial x}-\frac{c}{n_{0}} \widehat{E}\right)_{x=0}=b(t), \quad\left(\frac{\partial E}{\partial x}+\frac{c}{n_{0}} \widehat{E}\right)_{x=L}=0,
$$

where

$$
b(t)=-2 \frac{c}{n_{0}}\left(\widehat{E}_{i n}\right)_{x=0} .
$$

First, let us expand the total field in terms of QNMs

$$
\mathbf{E}(x, t)=\sum_{m=-\infty}^{\infty} a_{m}(t) \mathbf{Q}_{m}(x)
$$

Next, we project the total field onto the QNM basis

$$
\left\langle\mathbf{Q}_{k}, \mathbf{E}\right\rangle=\left\langle\mathbf{Q}_{k}, \sum_{m=-\infty}^{\infty} a_{m}(t) \mathbf{Q}_{m}\right\rangle
$$

Orthogonality of QNMs, invoked in the right-hand side gives

$$
\left\langle\mathbf{Q}_{k}, \mathbf{E}\right\rangle=a_{k}(t)\left\langle\mathbf{Q}_{k}, \mathbf{Q}_{k}\right\rangle .
$$

To obtain the time dynamics equation for the coefficients in the expansion, let us differentiate with respect to time

$$
\partial_{t} a_{k}(t)=\frac{\left\langle\mathbf{Q}_{k}, \partial_{t} \mathbf{E}\right\rangle}{\left\langle\mathbf{Q}_{k}, \mathbf{Q}_{k}\right\rangle}
$$


Using the time-evolution equation (2.15) this can be written

$$
\partial_{t} a_{k}(t)=\frac{\left\langle\mathbf{Q}_{k}, \mathcal{H} \mathbf{E}\right\rangle}{\left\langle\mathbf{Q}_{k}, \mathbf{Q}_{k}\right\rangle}
$$

According to the definition of $\mathcal{H}$, we have

$$
\mathcal{H} \mathbf{E}=\left(\frac{c^{2}}{n^{2}(x)} \widehat{E}, \partial_{x}^{2} E\right)^{T},
$$

and consequently in (2.51)

$$
\left\langle\mathbf{Q}_{k}, \mathcal{H} \mathbf{E}\right\rangle=\int_{0}^{L} d x\left(Q_{k} \partial_{x}^{2} E+\widehat{Q}_{k} \frac{c^{2}}{n^{2}(x)} \widehat{E}\right)+S_{t},
$$

where

$$
S_{t}=\frac{n_{0}}{c}\left(\left(Q_{k} \frac{c^{2}}{n_{0}^{2}} \widehat{E}\right)_{x=0}+\left(Q_{k} \frac{c^{2}}{n_{0}^{2}} \widehat{E}\right)_{x=L}\right) .
$$

Further, let us partially integrate twice in the first integral (2.54) and apply outgoing wave boundary conditions for the eigenfunctions and the transparent influx boundary conditions for the transmission field to obtain

$$
\left\langle\mathbf{Q}_{k}, \mathcal{H} \mathbf{E}\right\rangle=\int_{0}^{L} d x\left(E \partial_{x}^{2} Q_{k}+\widehat{Q}_{k} \frac{c^{2}}{n^{2}(x)} \widehat{E}\right)+S_{1}+S_{2}+S_{t}
$$

where

$$
S_{1}=\left.\left(Q_{k} \partial_{x} E\right)\right|_{0} ^{L}=-Q_{k}(0) b(t)-S_{t}
$$

and

$$
S_{2}=-\left.\left(E \partial_{x} Q_{k}\right)\right|_{0} ^{L}=\frac{n_{0}}{c}\left(\left(E \frac{c^{2}}{n_{0}^{2}} \widehat{Q}_{k}\right)_{x=0}+\left(E \frac{c^{2}}{n_{0}^{2}} \widehat{Q}_{k}\right)_{x=L}\right) .
$$

Upon identifying that

$$
\left\langle\mathcal{H} \mathbf{Q}_{k}, \mathbf{E}\right\rangle=\int_{0}^{L} d x\left(E \partial_{x}^{2} Q_{k}+\widehat{Q}_{k} \frac{c^{2}}{n^{2}(x)} \widehat{E}\right)+S_{2}
$$

follows the relation

$$
\left\langle\mathbf{Q}_{k}, \mathcal{H} \mathbf{E}\right\rangle=\left\langle\mathcal{H} \mathbf{Q}_{k}, \mathbf{E}\right\rangle-Q_{k}(0) b(t)
$$

After using the eigenvalue equation and orthogonality of QNMs from (2.52) the time- dynamics equation for the expansion coefficients reads

$$
\partial_{t} a_{k}(t)+i \omega_{k} a_{k}(t)=-\frac{Q_{k}(0) b(t)}{\left\langle\mathbf{Q}_{k}, \mathbf{Q}_{k}\right\rangle} .
$$


Therefore, in the presence of an incoming wave the coefficients in the expansion of the total field are determined as solutions of the inhomogeneous equation (2.61). If there are no incoming waves, the equation becomes homogeneous, as expected.

Note that in (2.61) the incoming wave excites all QNMs supported by the structure. However, a certain time interval after the excitation is withdrawn the time domain fields may be well approximated by one or few QNMs. This especially holds when defect QNMs with small imaginary parts are considered, see [26] and references therein.

Frequency domain To obtain a description of the system in the frequency domain let us perform a Fourier transform with $f(x, \omega)=\int_{-\infty}^{+\infty} f(x, t) e^{i \omega t} d t$, where $\omega \in \mathbb{R}$, on all quantities of interest. First, we take only the first component in the expansion formula (2.34) for the field inside the cavity:

$$
E(x, \omega)=\sum_{k=-\infty}^{\infty} a_{k}(\omega) Q_{k}(x) .
$$

Further, we Fourier transform the equation for the expansion coefficients (2.61) that include the contribution from the incoming wave (2.6) :

$$
a_{k}(\omega)=\frac{Q_{k}(0) b(\omega)}{i\left\langle\mathbf{Q}_{k}, \mathbf{Q}_{k}\right\rangle\left(\omega-\omega_{k}\right)}
$$

where

$$
b(\omega)=-2 \frac{c}{n_{0}} \widehat{E}_{i n}(0, \omega)=2 i \omega \frac{n_{0}}{c} E_{i n}(0, \omega) .
$$

From the continuity conditions for the field on the structure boundaries it follows that

$$
E(0, \omega)=E_{\text {in }}(0, \omega)+E_{s}(0, \omega)
$$

and

$$
E(L, \omega)=E_{t r}(L, \omega)
$$

Here $E_{s}$ represents the reflected, left traveling wave in the region $x<0, E_{t r}$ is the transmitted right traveling wave present for $x>L$. This enables us to relate the incident wave to the expanded field $E$ in the frequency domain. The amplitude transmission coefficient is defined as

$$
t(\omega)=\frac{E_{t r}(L, \omega)}{E_{i n}(0, \omega)}
$$

so we can write the expression for the transmission coefficient

$$
t(\omega)=2 \frac{n_{0}}{c} \sum_{k=-\infty}^{\infty} \frac{\omega Q_{k}(0) Q_{k}(L)}{\left\langle\mathbf{Q}_{k}, \mathbf{Q}_{k}\right\rangle\left(\omega-\omega_{k}\right)}
$$


If we apply the sum rule (2.35) the expression for the transmission coefficient obtains the form

$$
t(\omega)=2 \frac{n_{0}}{c} \sum_{k=-\infty}^{\infty} \frac{\omega_{k} Q_{k}(0) Q_{k}(L)}{\left\langle\mathbf{Q}_{k}, \mathbf{Q}_{k}\right\rangle\left(\omega-\omega_{k}\right)}
$$

which turns out to be the one with (much) faster convergence. A similar expression can be obtained for the reflection coefficient

$$
r(\omega)=\frac{E_{s}(0, \omega)}{E_{i n}(0, \omega)}=2 \frac{n_{0}}{c} \sum_{k=-\infty}^{\infty} \frac{\omega Q_{k}^{2}(0)}{\left\langle\mathbf{Q}_{k}, \mathbf{Q}_{k}\right\rangle\left(\omega-\omega_{k}\right)}-1
$$

The transmittance $T$ and reflectance $R$ (relative transmitted and reflected optical power) are given by

$$
T(\omega)=|t(\omega)|^{2}, \text { and } R(\omega)=|r(\omega)|^{2} .
$$
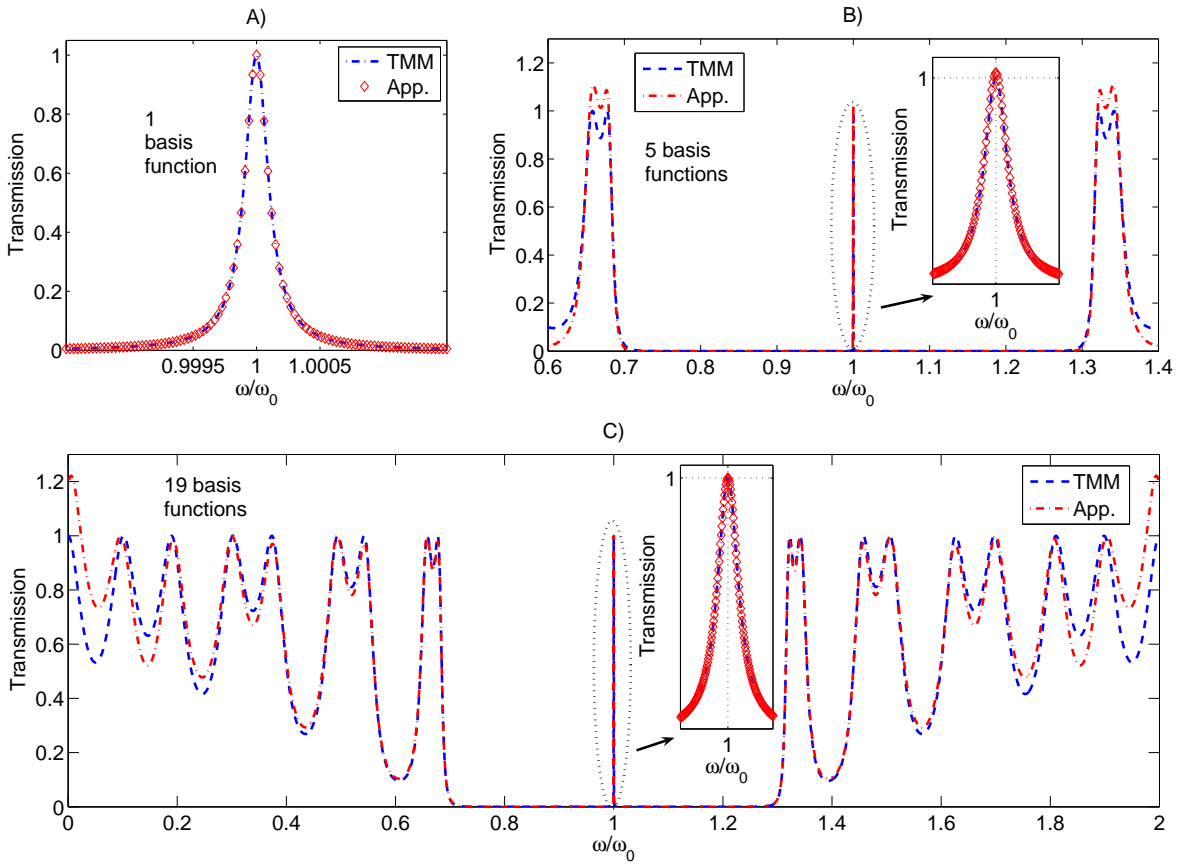

Figure 2.3: Spectral transmittance determined by (2.69) using different numbers of basis functions for periodic defect structure.

Consider the defect structure of section 2.1.2. Figure 2.3 compares the spectral transmittance (2.71) computed by TMM with the approximation (2.69) for different numbers of basis fields in expansion. In Figure $2.3 \mathrm{~A}$ ) the resonant transmission around the defect resonance is depicted. Good approximation of the spectral transmission is obtained with only one term, i.e. the contribution of the QNM with the 
real part of the eigenfrequency closest to the frequency of the defect resonance, see Figure 2.2 A). Naturally, it is expected that approximation improves when the number of basis functions, here the number of terms in (2.69), is increased to include contribution from other QNMs. Figure 2.3 B) depicts result including five QNMs, those associated with the defect and four of the bandedge eigenfrequencies. As can be seen the spectral transmission around the bandedge region is not adequate which should not be a surprising result as the contributions of other QNMs may not be negligible in these regions. However, as depicted in the inset of Figure 2.3 B), the approximation of the spectral transmission around the defect resonance gets worse. Further increasing the number of basis functions improves approximation of the spectral transmission in the whole frequency range and around the defect resonance. A result with 19 basis functions belonging to the first band (see Figure $2.2 \mathrm{~A}$ )) is shown in Figure $2.3 \mathrm{C}$ ). With larger number of basis functions the spectral transmission may be approximated up to the desired error. Similar examples of an approximation of the spectral transmission for periodic multilayers have been considered in $[65,72,79]$.
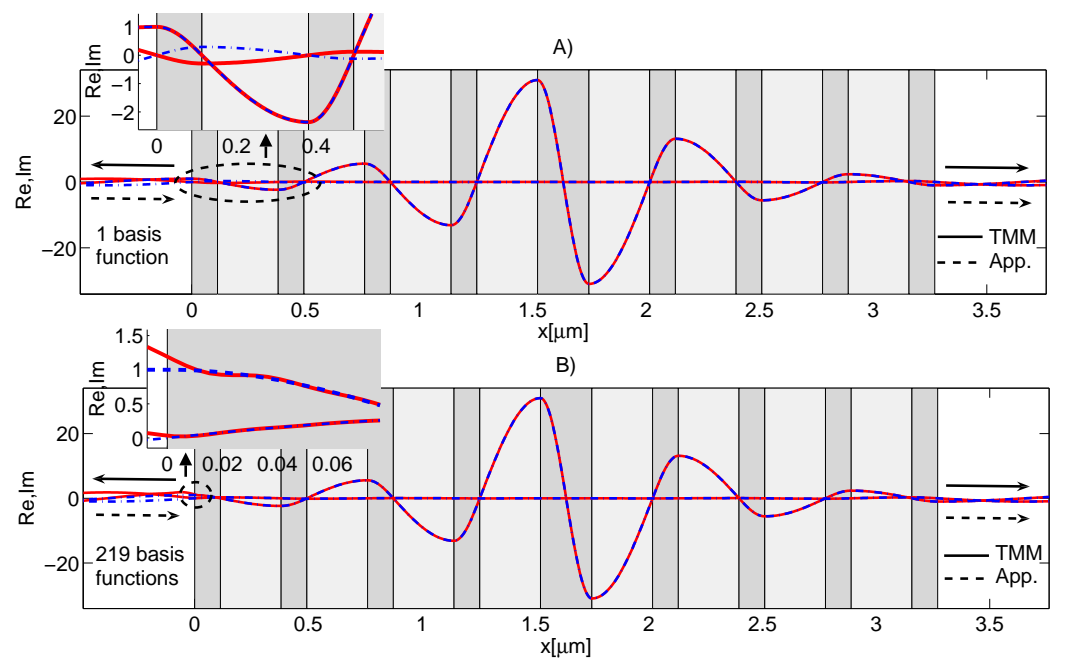

Figure 2.4: Field profile for transmission resonance; exact TMM solution and QNM expansion approximation.

The somewhat odd behavior of the QNM approximation may be understood better if the fields are considered directly. In Figure 2.4 we show comparisons between the QNM approximation and the TMM solution of the field profile associated with the defect resonance resonance $\omega=\omega_{0}$. Note that the transmission resonance in this configuration is a fully transmitted field because the structure possesses a spatially symmetric refractive index distribution. This is a somewhat extreme situation, because reflected waves (outgoing components of the total field) at the left side of the structure are not present. Therefore the nature and properties of QNMs are totally opposite to the fields that have to approximated. 
In Figure 2.4 A) both the TMM result and the QNM approximation with only one basis function is shown. It is clear that field is globally very well approximated when considered on the extension of the whole structure. However, as depicted in the inset fields are indeed different in a localized region. Around the input boundary this observation seems not to be entirely appreciated in the literature because usually comparisons of the modulus of the fields are considered. If the number of basis functions is increased the field approximation becomes better, and the localized region where the fields are different shrinks as shown in Figure $2.4 \mathrm{~A}$ ). Nevertheless, a characteristic oscillation of the approximated field around the exact solution (ringing artifact) demonstrates a form of the well known Gibbs phenomena [28]. This observation suggests that the QNM approximation may approach the exact solution only when an infinite number of the basis functions is included. Moreover, the approximation is valid only inside the structure up to the boundaries, see $[69,70,73]$ and references therein.

Note that the nature of QNMs and the related expansion method are somewhat special as the QNMs are solutions of a non-Hermitian eigenvalue problem. Then the completeness of the QNM basis, a uniqueness of the expansion, and convergence of truncated sums using QNMs can not be guaranteed by standard methods, see [24] and references therein. Indeed, [57, 77] point to the over-completeness of the QNM basis set. Therefore, coefficients in the QNM expansion may be determined in different ways. Moreover, as we already encountered during the derivation of the spectral transmission, e.g. the manipulations involving the summation rules (2.35),(2.36), special techniques have to be used to speed up the convergence of sums incorporating QNMs. Usually, for this purpose one explicitly uses similar re-summations of QNM expansion series according to the completeness relations [70].

The formalism using the pseudo-inner product as described previously may be appealing as if transcribes the derivation and form of the usual eigenfunction expansion technique. However, an alternative approach may be carried out without any reference to the completeness property or a pseudo-inner product if a variational form of the transmission problem and a field template with a truncated sum of QNMs from the whole set is used, see chapters 3 and 4. Then stationarity of the functional determines the relevant coefficients in the QNMs expansion.

\subsection{Time-independent perturbation theory for QNMs}

The previous results suggest that the QNMs supported by an optical structure contain all necessary information about intrinsic structural properties that control the response of the structure to some external influence. However, complexity of the QNMs computation for an arbitrary optical structure are a somewhat limiting factor for rapid and accurate analysis. Therefore, an perturbation theory for QNMs is of interest to provide an approach to computation of complex eigenfrequencies when internal perturbations of the structure are applied. 
After the results regarding the completeness property, the pseudo-inner product, and the two-component formalism, a time-independent perturbation theory for QNMs has been outlined in [70]. The formalism provides all derivations in close analogy to usual time-independent perturbation theory $[28,29]$. We briefly review this procedure in the following subsection.

\subsubsection{QNMs perturbation theory and two-component formalism}

A time-independent perturbation theory for QNMs can be derived from the twocomponent formalism [70]. Let us assume that some change of refractive index is represented as:

$$
\frac{1}{n_{\mathrm{pert}}^{2}}(x)=\frac{1}{n_{\mathrm{org}}^{2}}(x)(1+\mu V(x))
$$

where $\mu$ is the (small) parameter related to the change of refractive index in the structure. The perturbation $V(x)$ is nonzero only inside the computational domain $x \in[0, L]$. The eigenvalue problem for the perturbed structure is

$$
\left(\mathcal{H}^{(0)}+\mu \Lambda\right) \mathbf{Q}_{k}^{(p)}=\left(-i \omega_{k}^{(p)}\right) \mathbf{Q}_{k}^{(p)},
$$

with the operator of the original (unperturbed system)

$$
\mathcal{H}^{(0)}=\left(\begin{array}{cc}
0 & \frac{c^{2}}{n_{\mathrm{org}}^{2}(x)} \\
\partial_{x}^{2} & 0
\end{array}\right),
$$

and the perturbation operator

$$
\Lambda=\left(\begin{array}{cc}
0 & \frac{c^{2}}{n_{\mathrm{org}}^{2}(x)} V(x) \\
0 & 0
\end{array}\right) .
$$

Let us assume that the unperturbed eigenvalue problem is solved and eigenvalues and eigenfunctions are known

$$
\left(\mathcal{H}^{(0)}\right) \mathbf{Q}_{k}^{(0)}=\left(-i \omega_{k}^{(0)}\right) \mathbf{Q}_{k}^{(0)}
$$

Then, perturbation theory seeks for solutions of the eigenvalue problem with eigenvalues and eigenfunctions expressed as a power series in the small parameter:

$$
\omega_{k}^{p}=\sum_{j=0}^{\infty}\left(\mu^{j}\right) \omega_{k}^{(j)}
$$

and

$$
\mathbf{Q}_{k}^{(p)}=\sum_{j=0}^{\infty}\left(\mu^{j}\right) \mathbf{Q}_{k}^{(j)}
$$


Next, we substitute (2.77) and (2.78) into (2.73) and group terms with the same order in $\mu$ to get:

$$
\begin{gathered}
\mathcal{H}^{(0)} \mathbf{Q}_{k}^{(0)}=\left(-i \omega_{k}^{(0)}\right) \mathbf{Q}_{k}^{(0)} \\
\left(\mathcal{H}^{(0)}-\left(-i \omega_{k}^{(0)}\right)\right) \mathbf{Q}_{k}^{(1)}+\left(\Lambda-\left(-i \omega_{k}^{(1)}\right)\right) \mathbf{Q}_{k}^{(0)}=0
\end{gathered}
$$

which are the terms up to the first order in $\mu$. Let us project equation (2.80) onto the eigenfunction of the unperturbed system $\mathbf{Q}_{m}^{(0)}$ :

$$
\left\langle\mathbf{Q}_{m}^{(0)},\left(\mathcal{H}^{(0)}-\left(-i \omega_{k}^{(0)}\right)\right) \mathbf{Q}_{k}^{(1)}\right\rangle+\left\langle\mathbf{Q}_{m}^{(0)},\left(\Lambda-\left(-i \omega_{k}^{(1)}\right)\right) \mathbf{Q}_{k}^{(0)}\right\rangle=0
$$

Due to symmetry of the $\mathcal{H}^{(0)}$ and orthogonality of the eigenfunctions the first term vanishes and from the second term it follows that

$$
\left\langle\mathbf{Q}_{k}^{(0)}, \Lambda \mathbf{Q}_{k}^{(0)}\right\rangle=\left(-i \omega_{k}^{(1)}\right)\left\langle\mathbf{Q}_{k}^{(0)}, \mathbf{Q}_{k}^{(0)}\right\rangle .
$$

Therefore, the first order correction to the complex frequency is given in the form

$$
\omega_{k}^{(1)}=i \frac{\left\langle\mathbf{Q}_{k}^{(0)}, \Lambda \mathbf{Q}_{k}^{(0)}\right\rangle}{\left\langle\mathbf{Q}_{k}^{(0)}, \mathbf{Q}_{k}^{(0)}\right\rangle},
$$

which gives first order corrections of the real and complex parts of the frequency. The first order eigenvalue correction requires only the respective eigenvalue and eigenfunction of the unperturbed system, while higher order corrections include summations over all QNMs [70].

Although this approach is similar in form to the usual perturbation theory [28], it has been scarcely applied for the concrete analysis of general structures. This is partly because it relies on the completeness property and partly due to fact that higher order approximations obtained are slowly convergent and require special acceleration methods to obtain faster converging series [70].

\subsubsection{Variational QNM perturbation theory}

A path toward a perturbation theory for QNMs that does not rely on the completeness of the QNMs basis set or the pseudo-inner product is to use the variational formulation of QNMs (eigenvalue) problem, see chapter 4. One can start with the functional

$$
\mathcal{L}_{\omega}(Q)=\frac{1}{2} \int_{0}^{L}\left(\left(\partial_{x} Q(x)\right)^{2}-\frac{\omega^{2} n^{2}(x)}{c^{2}} Q^{2}(x)\right) d x-\frac{i \omega n_{0}}{2 c}\left(\left.Q^{2}\right|_{x=0}+\left.Q^{2}\right|_{x=L}\right) .
$$

If $\mathcal{L}$ becomes stationary, i.e. if the first variation of $\mathcal{L}$ vanishes for arbitrary variations of $Q$, then $Q$ satisfies the Euler-Lagrange equation (2.3), and (2.4) as natural boundary conditions. 
Consider a perturbation of the structure that can be expressed as a local perturbations of the permittivity $n^{2}(x)=n_{\text {org }}^{2}(x)+n_{p t}^{2}(x)$ for $x \in[0, L]$. Let us assume that $\left(\omega_{0}, Q_{0}\right)$ is the known solution of the QNM problem for the unperturbed structure with permittivity $n_{\text {org }}^{2}$. The value of the functional for the eigenfunctioneigenvalue pair is zero $\mathcal{L}_{\omega_{0}}\left(Q_{0}\right)=0$. This property that can be shown by integrating the first term in the (2.84) by parts.

Small in effect perturbations of the original structure should not change greatly neither the position of the complex eigenfrequency in the complex plane nor the shape of the corresponding QNM. Let the exact solution for the perturbed structure be $(\omega, Q)$ with eigenfrequency $\omega=\omega_{0}+\delta \omega$. It can be easily shown that the restriction to the $\mathrm{QNM}$ solution of the unperturbed problem $Q \simeq Q_{0}$ and the stationarity of the functional leads to

$$
\left(\omega_{0}+\delta \omega\right)^{2}\left(\mathcal{A}_{0}\left(Q_{0}\right)+\mathcal{A}_{p t}\left(Q_{0}\right)\right)+\left(\omega_{0}+\delta \omega\right) \mathcal{B}_{0}\left(Q_{0}\right)+\mathcal{C}_{0}\left(Q_{0}\right) \simeq 0
$$

where

$$
\begin{gathered}
\mathcal{A}_{0}\left(Q_{0}\right)=-\frac{1}{2 c^{2}} \int_{0}^{L} n^{2}(x) Q_{0}^{2}(x) d x, \\
\mathcal{A}_{p t}\left(Q_{0}\right)=-\frac{1}{2 c^{2}} \int_{0}^{L} n_{p t}^{2}(x) Q_{0}^{2}(x) d x, \\
\mathcal{B}_{0}(Q)=-\frac{i}{2 c}\left(\left.n_{\text {in }} Q^{2}\right|_{x=0}+\left.n_{\text {out }} Q^{2}\right|_{x=L}\right), \\
\mathcal{C}_{0}(Q)=\int_{0}^{L}\left(\partial_{x} Q(x)\right)^{2} d x .
\end{gathered}
$$

After evaluating (2.85), the correction to the eigenvalue up to first order reads

$$
\delta \omega=-\frac{\frac{\omega_{0}^{2}}{c^{2}} \int_{0}^{L} n_{p t}^{2}(x) Q_{0}^{2}(x) d x}{\left.\frac{2 \omega_{0}}{c^{2}} \int_{0}^{L} n_{\text {org }}^{2}(x) Q_{0}^{2} d x+\frac{i n_{0}}{c}\left(\left.Q_{0}^{2}\right|_{x=0}+\left.Q_{0}^{2}\right|_{x=L}\right)\right)} .
$$

Consider the example of single cavity structure coded as $(H L)^{4} D(L H)^{4}$, with $n_{H}=3.42 n_{L}=1.45$, enclosed within two semiinfinite media of the same refractive index $n_{0}=1.0$. All layers are quarter-wavelength, except the central defect layer that is half-wavelength. Perturbation of this structure is a localized change of the defect layer refractive index $n^{2}=n_{D}^{2}(1 \pm \chi)$ with $\chi \in(-0.1,0.1)$. The thicknesses and refractive indices of other layers are not affected.

Figure $2.5 \mathrm{~A}$ ) compares positions of the complex eigenfrequencies for the perturbed structure computed according to the first order perturbation theory formula (2.90) with the direct computation. Note that the perturbation theory correction is tangent to the eigenfrequency path in the complex plane. In Figure $2.5 \mathrm{~B}$ ) and C) the dependence of the real and imaginary parts of the complex eigenfrequency on the defect layer refractive index is shown.

The variational perturbation theory may be extended to higher order corrections. Then variational accuracy guarantees that certain order of the perturbed 

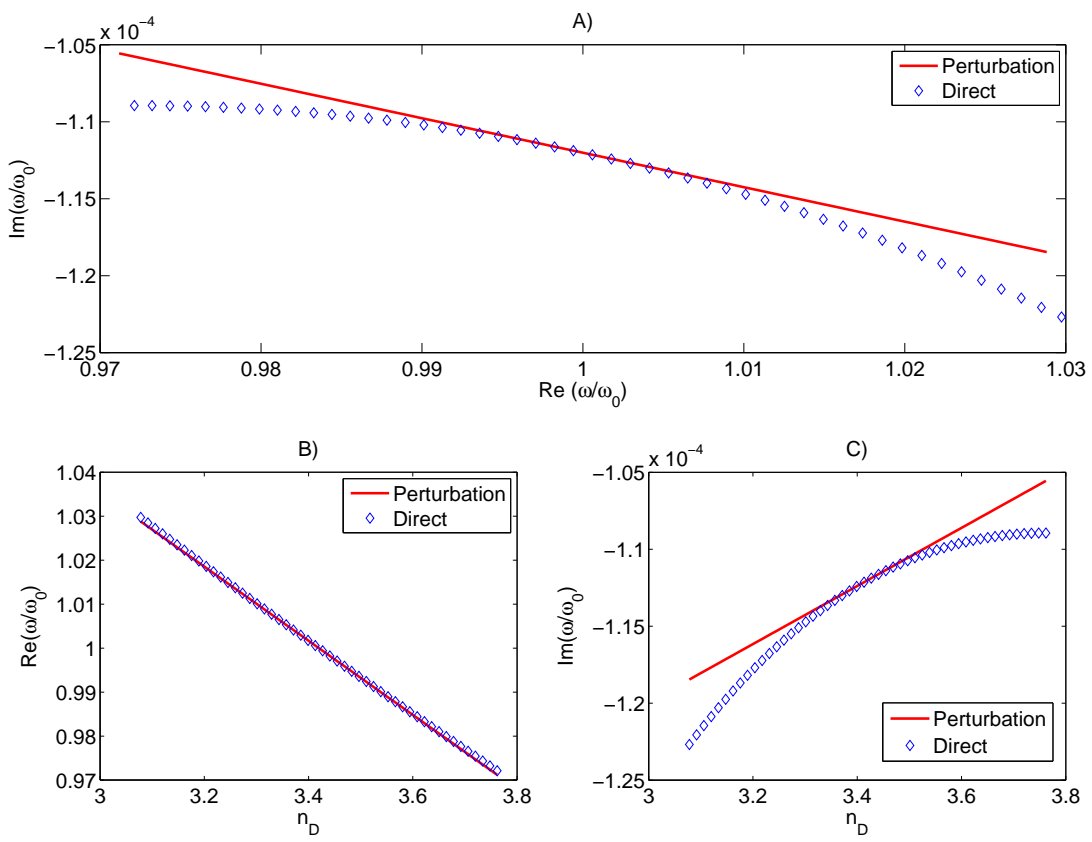

Figure 2.5: Complex eigenfrequencies for the single-defect structure obtained by direct computation of eigenvalues for the perturbed structure, and by first order perturbation theory .

eigenfrequency is determined by lower order eigenfunctions. Using subsequently suitable restrictions and the stationarity of the functional, an iterative procedure for determining higher orders correction to the eigenfrequencies and QNMs may be obtained. 


\title{
Chapter 3
}

\section{Field representation for optical defect resonances in multilayer microcavities using quasi-normal modes}

\begin{abstract}
${ }^{1}$
Quasi-Normal Modes are used to characterize transmission resonances in $1 D$ optical defect cavities and the related field approximations. We specialize to resonances inside the bandgap of the periodic multilayer mirrors that enclose the defect cavities. Using a template with the most relevant QNMs a variational principle permits to represent the field and the spectral transmission close to resonances.
\end{abstract}

\footnotetext{
${ }^{1}$ This chapter is adapted from: M.Maksimovic, M. Hammer, E.van Groesen, Field representation for optical defect resonances in multilayer microcavities using quasi-normal modes, Optics Communications, 281, 1401-1401, 2008
} 


\subsection{Introduction}

When subjected to external excitation, periodic multilayer structures show a resonant response in the time or frequency domain, which can be tailored by inclusion of defects $[8,7,9,4]$. Finite structures can be viewed as open systems which permit the leakage of energy to the exterior, described by the Helmholtz equation with outgoing wave boundary conditions. This constitutes an eigenvalue problem for complex frequencies and the associated field profiles, or quasi-normal modes (QNMs) [20].The quasi-normal modes specify the field patterns in which the leaky optical structure would oscillate naturally after an initial excitation is withdrawn, representing damped oscillatory solutions of the wave equation [24, 58]. QNMs and associated complex eigenvalues can be viewed as a proper model for solving the problem of energy leaking out of open structures, see [7, 20, 24, 26] and references therein.

Our aim is to use the characterization of the optical microcavity structures in terms of quasi-normal modes to describe approximately the resonant response to external excitation in the frequency domain and the related field profiles.

For specific configurations the complex QNM eigenvalues appear to correspond to the position and quality of resonances in the spectral transmission. Although this holds when spectral resonances are sufficiently far apart from each other, this is not a general property and real frequencies of transmission resonances can be quite different form the real parts of the complex QNM eigenfrequencies in the given frequency range [79].

Field representations using QNMs have been investigated in $[79,65,71,72$, 80], on the basis of quasi-normal mode theory as established in [24], founded on certain completeness properties and a linear-space structure for QNMs [68, 69, 70]. Orthogonality of QNMs is expressed with a specific bilinear form that includes boundary terms and in contrast to the usual inner product does not define a real, positive definite norm $[74,75]$. An eigenfunction expansion based on this bilinear form [24, 73], used as a means for projecting functions onto the QNM basis, can furnish a field representation only over a finite spatial domain (due to exponentially growing envelopes of QNM basis functions) and under certain conditions necessary for completeness, as detailed in [68]. The completeness properties of QNMs have been addressed also in [76, 77].

When applying a QNM expansion method to transmission problems, several points are important. First, individual QNMs do not satisfy the proper boundary conditions for the transmittance problem directly. The incoming wave contribution in the transmission problem is introduced via time dynamic equations for the expansion coefficients. Frequency domain equations are obtained by Fourier transform [72], [73]. Second, as detailed in reference [73], an expansion based solely on QNMs can represent the internal cavity field up to the boundary of the enclosed region, with exception of a set of measure zero (the boundary points). This means that QNM expansions permit convergence in the mean but not pointwise. This situation arises specifically when the relevant field does not satisfy the same outgoing 
wave boundary conditions as the QNMs. Hence, despite the completeness property, in these cases finite, truncated QNM expansions lead to poorly converging field representations. Only after applying certain summation rules following from the completeness relations [24, 73] a better convergence might be achieved. Still, adequate approximations of the fields usually require summations over many basis modes (although this hardly ever seems to have been observed explicitly, perhaps due to comparisons of intensity shapes in place of field profiles). In addition some caution is necessary when taking into account contributions of a single resonance in the field representation based on QNM expansion, as emphasized in the reference [73]. This holds even in a spectral region of isolated, defect-induced transmission resonances in the bandgap, where one would expect that only those QNMs with the real parts of their eigenfrequencies in this frequency region are sufficient.

Alternative approaches in describing leaky optical structures are reported in literature and field representations in open 1D cavity structures are considered, see [58] and references therein. These methods primarily consider quantum theory of open systems and do not establish a direct connection between transmittance (scattering) problems and QNMs for general structure.

As far as we are aware no adequate generalizations to $2 \mathrm{D}$ and $3 \mathrm{D}$ structures are reported in literature of the above mentioned approaches. This is also true for PBG (photonic bandgap) structures that we are primarily interested in. Some attempts to use a QNM-like (eigenmode) description for 2D and 3D PBG structures are those related to a scattering-matrix approach as reported in [81, 82] and [23].

It is the purpose of this paper to establish a quantitative relation between the description of the structure under external excitation with given fixed frequency (the transmittance problem) and the eigenvalue problem for QNMs, emphasizing the nature of realistic open structures. Our method specializes to optical defect structures where high-Q resonances are present inside the photonic bandgap. As detailed in this paper, it turns out that the variational form of the transmission problem offers a resourceful alternative to existing methods when applied to description of the fields and transmission responses of the localized defect modes formed inside the photonic bandgap. Our method does not rely on any completeness properties of QNMs nor on a bilinear form for projecting fields onto the QNM basis.

The approach proposed in section 2 uses a combination of the bandgap field of the structure (without defect) and only one/few relevant QNM(s) as a template. By restricting a specific functional one obtains approximations for the spectral power transfer and the optical field related to the transmission through the defect structure. In section 3 we analyze single and multiple defect cavities in finite 1D periodic structures for both symmetric and non-symmetric layer arrangements. Real world structures are bound to be finite and this feature is explicitly incorporated by the present approach, in contrast to techniques that rely on artificial periodization in Bloch-type analysis and supercell methods that can introduce nonphysical and spurious solutions, although usefulness and applicability of these methods is proven and well established in practice [7, 9]. 


\subsection{Theory}

We consider problems in 1D for structures with arbitrary piecewise constant refractive index distribution $n(x)$ within a finite domain $x \in(0, L)$ and assume that the structure is enclosed by two semi infinite domains with constant refractive indices $n_{\text {in }}, n_{\text {out }}$ as depicted in Fig. 1 .

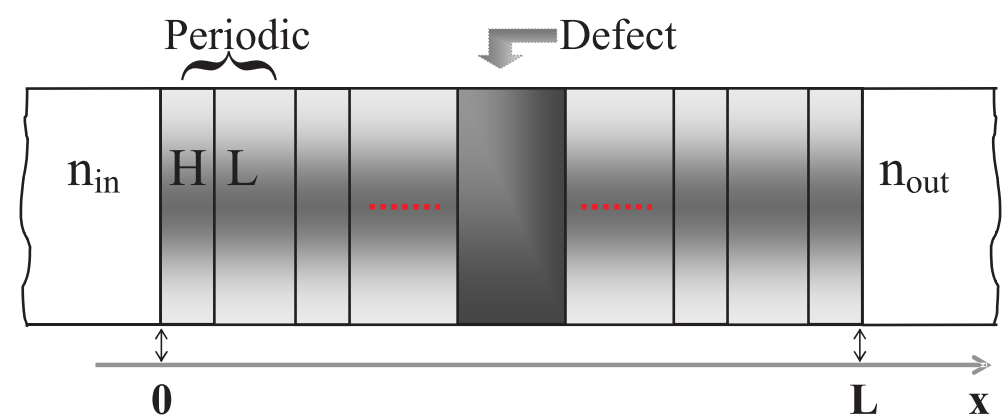

Figure 3.1: The (defect) grating is a finite periodic structure consisting of two materials with high index $n_{H}$ and low index $n_{L}$. The layer thicknesses $L_{H}, L_{L}$ are quarter-wavelength for the target wavelength. Optical defects are introduced as changes of layer thicknesses. The grating is surrounded by two semi-infinite media of indices $n_{\text {in }}$ and $n_{\text {out }}$.

We choose a harmonic time dependence for the electric field

$$
E(x, t)=E(x) e^{-i \omega t}
$$

Therefore, the response of the structure under external excitation is described by the Helmholtz equation

$$
\partial_{x}^{2} E(x)+k^{2}(x) E(x)=0
$$

with a transparent influx boundary condition at the side of the structure where the incident wave $\left(E_{i n c}=A_{i n c} e^{i k_{i n} x}\right)$ impinges, and a transparent boundary condition at the other side

$$
\begin{gathered}
\left.\left(\partial_{x}+i k_{i n}\right) E\right|_{x=0}=2 i k_{i n} A_{i n c}, \\
\left.\left(\partial_{x}-i k_{\text {out }}\right) E\right|_{x=L}=0
\end{gathered}
$$

where $k(x)=\omega^{2} n^{2}(x) / c^{2}, k_{i n}=n_{i n} \omega / c$ and $k_{\text {out }}=n_{\text {out }} \omega / c$, for given amplitude of the incident wave $A_{i n c}$ and frequency $\omega$. This is the transmittance problem, where the field distribution inside and outside the structure for given real frequency $\omega$ of the incident wave is to be determined. For a solution of (3.2),(3.3),(3.4) the transmittance is the ratio between the time-averaged Poynting vectors in the respective media of incident and output regions. This is the ratio between incident 
and transmitted power for time-harmonic electromagnetic fields

$$
T=\frac{P_{\text {out }}}{P_{\text {in }}}=\frac{\frac{1}{2} n_{\text {out }}|E(L)|^{2}}{\frac{1}{2} n_{\text {in }}\left|E_{\text {inc }}(0)\right|^{2}} .
$$

In this context, a transmission resonance can be defined as a local maximum of the transmittance in a selected frequency region of otherwise low transmittance.

Alternatively, a finite structure can be viewed as an open system with transparent boundaries which permit leakage of energy to the exterior. Just as before, the behavior of the electric field in the interior $x \in(0, L)$ is governed by the Helmholtz equation

$$
\partial_{x}^{2} Q(x)+k^{2}(x) Q(x)=0
$$

now with outgoing wave boundary conditions

$$
\begin{gathered}
\left(\partial_{x} Q(x)+i k_{i n} Q(x)\right)_{x=0}=0 \\
\left(\partial_{x} Q(x)-i k_{\text {out }} Q(x)\right)_{x=L}=0 .
\end{gathered}
$$

This constitutes an eigenvalue problem for the frequency $\omega$ as the complex eigenvalue and the electric field $Q(x)$ as eigenfunction, called Quasi-Normal Mode. QNMs appear as discrete infinitely countable set of solutions of this eigenvalue problem [24]. They are unbounded functions that blow-up for $x \rightarrow \pm \infty$, so they are essentially different from resonant field solutions of the transmittance problem.

A finite, but internally periodic structure, i.e. a finite multilayer grating possesses a QNM spectrum that appears to be related to the bandgap structure and resonance properties of the transmission / reflection spectra. Representations in terms of QNMs for finite, periodic structures have been investigated in $[65,71,72]$. The positions of complex eigenfrequencies in the complex plane are arranged in such way that suggest the presence of bandgap regions in the transmittance response. Occurrence of the bandgap is to be expected for slices in the complex frequency plane where eigenvalues are not present. The edge of the bandgap in these terms can be estimated by taking real parts of the eigenfrequencies at the ends of separated arranged sets of eigenvalues $[65,71,72]$. The introduction of a defect in an otherwise periodic multilayer results in isolated QNMs with the real parts of their complex eigenfrequencies inside the bandgap region, as shown in section 3.

In $[65,72]$ it has been noticed that the squared modulus of a QNM with complex frequency $\omega_{p}$ is similar to the field intensity inside the structure for a real frequency $\omega \approx \operatorname{Re}\left(\omega_{p}\right)$. This is a good approximation in particular for high-Q transmission resonances and for QNMs with eigenfrequencies with small imaginary parts. Still, a proper approximation of the field (not intensity) in the transmission problem requires many terms in an expansion based solely on QNMs.

\subsubsection{Solutions by transfer-matrix method}

We consider multilayer structures with piecewise constant refractive index distribution inside the finite spatial domain. Method for solving the transmittance (and 
eigenvalue) problems is the well known transfer matrix method (TMM) [4]. Solutions of the Helmholtz equation are given as combinations of left- and righttravelling waves in the $j$-th layer

$$
E_{j}(x)=A_{j} e^{i k_{j}\left(x-l_{j-1}\right)}+B_{j} e^{-i k_{j}\left(x-l_{j-1}\right)}
$$

for $x \in\left[l_{j-1}, l_{j}\right]$ in a region of constant index $n_{j}$ where $k_{j}=n_{j} \omega / c$ is the wave number in this layer. To connect the fields inside all layers we impose continuity conditions at the interfaces between consecutive layers,

$$
E_{j}\left(l_{j}\right)=E_{j+1}\left(l_{j}\right),
$$

and

$$
\partial_{x} E_{j}\left(l_{j}\right)=\partial_{x} E_{j+1}\left(l_{j}\right) .
$$

These conditions lead to a system of equations that relate amplitudes of leftand right- traveling waves in different layers. They can be represented in matrix form. Ordered multiplication of the relevant matrices connects amplitudes in each layer of the structure, as well as the amplitudes in the incidence and output regions:

$$
\left(\begin{array}{c}
A_{\text {in }} \\
B_{\text {in }}
\end{array}\right)=\left(\begin{array}{ll}
m_{11}(\omega) & m_{12}(\omega) \\
m_{21}(\omega) & m_{22}(\omega)
\end{array}\right)\left(\begin{array}{c}
A_{\text {out }} \\
B_{\text {out }}
\end{array}\right)
$$

The transmittance problem with incoming wave from the left is solved with $B_{\text {out }}=$ 0 for specified $A_{i n}$ (amplitude of the incoming wave) with given real frequency $\omega \in R$. The amplitude transmission and reflection coefficients are expressed as

$$
\begin{aligned}
& t(\omega)=\frac{A_{\text {out }}}{A_{\text {in }}}, \\
& r(\omega)=\frac{B_{\text {in }}}{A_{\text {in }}} .
\end{aligned}
$$

If we choose conditions $A_{\text {in }}=B_{\text {out }}=0$, i.e. restrict the exterior solutions to purely outgoing waves, the eigenvalue problem with outgoing wave boundary conditions is addressed. With these conditions the system of equations can be nontrivially satisfied if

$$
m_{11}(\omega)=0 .
$$

Analytic continuation of transfer matrix into the complex plane enables us to find solutions of (3.15) as complex eigenvalues $\omega$ [23]. By substituting the eigenvalue into the field representation (3.9) we obtain the corresponding eigenfunction, up to a complex constant. To solve (3.15) we apply a numerical iteration procedure of Newton type. In cases when that method fails to converge due to closely spaced eigenvalues, we use a more powerful technique for determining complex solutions, based on the argument principle method from complex analysis [81]. 


\subsubsection{Field template and variational formulation for transmittance problem}

We specialize to finite periodic structures that possess transmission properties with a bandgap, i.e. with a region of frequencies of very low transmission. Introduction of suitable defects leads to a resonant transmission response inside the bandgap of the underlying periodic structure. We choose a field template for the transmittance problem as

$$
E(x, \omega) \simeq E_{m f}(x, \omega)+\sum_{p=1}^{N} a_{p}(\omega) Q_{p}(x),
$$

where $p$ is an index counting $N$ relevant QNMs . We take the relevant QNMs as those with the real part of their complex frequency in the given frequency range. We show, in terms of the successful application of the template (3.16), that the transmission resonance associated with the defect, appearing inside the bandgap, is triggered by the "mirror" field $E_{m f}$ of the periodic structure without defect, which for frequencies inside the bandgap is an almost completely reflected wave with only a weak tail that extends into the interior of the structure.

Note that $E_{m f}$ satisfies correct boundary conditions of the form (3.3) for the transmittance problem, while a superposition of QNMs can not cover the contribution of the incoming wave directly. The incoming field has to be included by other means when expansion into the complete set of QNMs is considered [72]. The inclusion of $E_{m f}$ (or some similar object) is essential in our approach as a means to represent the incoming wave. The mirror field does not extend far into the region of the defect where the contributions of the relevant QNMs are expected to be dominant.

Hence, according to template (3.16) the forced resonance response of the structure appears because the incident wave possesses a real frequency close to the real part of the complex eigenfrequency of a suitable QNM supported by the defect structure.

Obviously (3.16) constitutes only an approximate model for the transmittance problem in specific frequency regions, since neither $E_{m f}$ nor $Q$ satisfy all of equations (3.2)-(3.4). The residuals can be viewed as contributions from other QNMs in the complete set supported [68] by the defect structure, that are not included in (3.16). We shall see, however, that the template (3.16) leads to excellent approximations for the configurations of section 3 .

To determine the decomposition coefficients $a_{p}$ in our field template we employ a variational form of the transmittance problem. The transmittance problem corresponds to the equation and natural boundary conditions, arising from the condition of stationarity of the functional [25]:

$$
\begin{aligned}
L(E) & =\int_{0}^{L} \frac{1}{2}\left(\left(\partial_{x} E(x)\right)^{2}-k^{2}(x) E^{2}(x)\right) d x \\
& -\frac{1}{2} i k_{i n} E^{2}(0)-\frac{1}{2} i k_{\text {out }} E^{2}(L)+2 i k_{\text {in }} A_{\text {inc }} E(0) .
\end{aligned}
$$


If $L$ becomes stationary, i.e. if the first variation of $L(E)$ vanishes for arbitrary variations of $E$, then $E$ satisfies (3.2), (3.3), and (3.4) as natural boundary conditions ${ }^{1}$.

Restricting the functional (3.17) to our field template (3.16), $L$ becomes a function of the coefficients $a_{p}$, for given $E_{m f}$ and $Q_{p}$. The stationarity conditions then read:

$$
\frac{\partial L\left(a_{1}, a_{2}, \ldots, a_{N}\right)}{\partial a_{q}}=0, q=1, \ldots, N .
$$

The optimal coefficients can be obtained as solutions of a system of linear equations

$$
\mathbf{A} \cdot \mathbf{a}=-\mathbf{b},
$$

where $\mathbf{a}=\left[a_{1}, a_{2}, \ldots, a_{N}\right]^{T}$ is the vector of coefficients to be determined. The components of the matrix $\mathbf{A}=\left[A_{q p}\right]_{N x N}$ and vector $\mathbf{b}=\left[b_{1}, . ., b_{N}\right]^{T}$ are

$$
\begin{aligned}
A_{q p} & =\int_{0}^{L}\left(\partial_{x} Q_{q} \partial_{x} Q_{p}-\frac{n^{2}(x) \omega^{2}}{c^{2}} Q_{q} Q_{p}\right) d x \\
& +i \frac{\omega}{c}\left(n_{\text {in }} Q_{q}(0) Q_{p}(0)+n_{\text {out }} Q_{q}(L) Q_{p}(L)\right)
\end{aligned}
$$

and

$$
\begin{aligned}
b_{q} & =\int_{0}^{L}\left(\partial_{x} E_{m f} \partial_{x} Q_{q}-\frac{n^{2}(x) \omega^{2}}{c^{2}} E_{m f} Q_{q}\right) d x \\
& +i \frac{\left(\omega_{q}-\omega\right)}{c}\left(n_{\text {in }} E_{m f}(0) Q_{q}(0)+n_{\text {out }} E_{m f}(L) Q_{q}(L)\right)+2 i n_{\text {out }} \frac{\omega}{c} A_{\text {inc }} Q_{q}(0) .
\end{aligned}
$$

By solving the system of equations (3.19) for each value of the real frequency $\omega$ the decomposition coefficients in the field representation for the transmittance problem are obtained. This enables approximation of the spectral transmittance and reflectance and the related field profile. The transmittance reads:

$$
T(\omega)=\frac{n_{\text {out }}}{n_{\text {in }}} t t^{*}=\frac{n_{\text {out }}}{n_{\text {in }}}\left|\frac{E_{m f}(L)+\sum_{p=1}^{N} a_{p}(\omega) Q_{p}(L)}{A_{\text {inc }}}\right|^{2} .
$$

The field in the region of incidence can be seen as a superposition of incident and reflected waves

$$
E(x)=A_{i n c} e^{i k_{i n} x}+r A_{i n c} e^{-i k_{i n} x},
$$

where $r$ is the amplitude reflection coefficient, related to the reflectance (power reflection defined as the ratio between the Poynting vectors of reflected and incident waves)

$$
R=r r^{*}=\left|\frac{E_{m f}(0)+\sum_{p=1}^{M} a_{p}(\omega) Q_{p}(0)}{A_{\text {inc }}}-1\right|^{2} .
$$

\footnotetext{
${ }^{1}$ The functional obtained from (3.17) by setting $A_{i n c}=0$, is formally related to the bilinear form (inner product), in which QNMs are orthogonal [24]. However, the frequency plays a different role in both cases: while in the functional (3.17) it is a given parameter, the bilinear form of [24] operates on objects that include the eigenfrequencies as arguments.
} 


\subsection{Results and Discussion}

We specialize to structures with piecewise constant refractive index distribution of high $n_{H}$ and low refractive index $n_{L}$ layers, with quarter-wavelength optical thicknesses $L_{H}, L_{L}$ at a target frequency $\omega_{0}$. High index layers are denoted by $H$, low-index layers by $L$ and defect layers by $D$. Thus a finite symmetric periodic structure is represented by $(H L)^{N} H$, where $N$ is the number of the layer pairs. The defects are introduced as changes of the thickness of specific layers, but the method can handle defects introduced as changes of refractive indices of certain layers as well. In examples below we use only 1, 2 and 3 most relevant QNMs corresponding to single-, double- and triple-cavity structures.

When single high- $\mathrm{Q}$ resonances inside the bandgap are considered, decomposition coefficients depend weakly on the frequency, apart from the Lorentzian approximation (eqns. $(3.28,3.29)$ ). Then the transmittance profile can be obtained analytically for the major part of the bandgap region around resonance position. If the full frequency dependence is included in $E_{m f}$ as described so far, then computational cost is comparable to direct TMM computations for the full structure, but captures adequately the deviation from the Lorentzian approximation. Further, we earn a certain degree of interpretability by being able to observe the interplay between the QNM basis modes.

\subsubsection{Symmetric single cavity structure}

We consider a layer arrangement $(H L)^{4} D(L H)^{4}$, as an example of a single cavity, with $n_{H}=3.42 n_{L}=1.45$, enclosed within two semiinfinite media of the same refractive index $n_{\text {in }}=n_{\text {out }}=1.0$. The defect is introduced as a change of the thickness $L_{H}$ in the central layer with high refractive index $n_{H}$ with $L_{D}=2 L_{H}$.

The QNM spectrum for the original periodic structure and the structure with the defect is depicted in Fig. 2A). The QNM frequencies clearly show an arrangement in the complex plane, that reflects the presence of the bandgap in the transmittance response presented in Fig. 2B). For the defect structure a complex frequency in the QNM spectrum appears in the position of the transmission resonance in the bandgap (see Fig. 2A). The field profile of the transmission resonance and the profile of the QNM corresponding to the defect structure have similar pattern as depicted in Figs. 2 C) and D). The difference between the QNM and transmission resonance field is clearly visible in Fig. 2E), arising from different boundary conditions (around $x=0$, the transmittance field represents inwards traveling wave and QNM outwards traveling wave), consequently leading to entirely different behavior in the region where the incident wave is present. We take the mirror field in the template as the solution of the transmittance problem of the structure without defect at each frequency in the considered bandgap region.

Approximating both the mode structure and the correct boundary conditions for the field representation of the TRM (transmission resonance mode Fig. 2C), using the QNMs and the mirror field in Fig. 2F) leads to an excellent agreement 

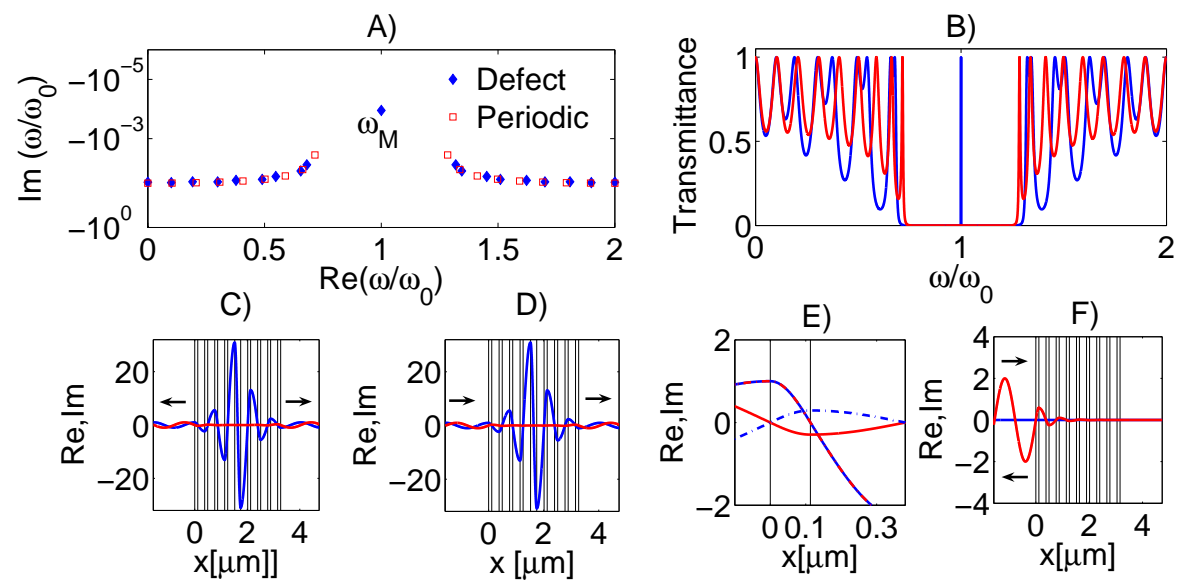

G)

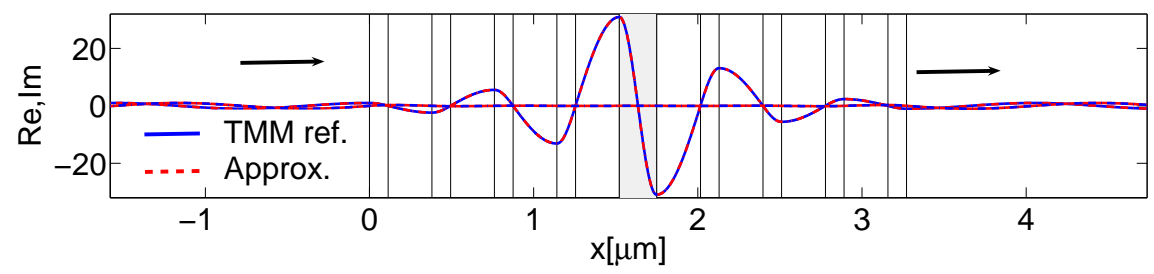

Figure 3.2: A) Complex frequencies (eigenvalues) for periodic and single cavity structure B) Transmittance for periodic (dashed) and single cavity structure (continuous) C) Field pattern for a (defect) frequency at the center of the bandgap, real and imaginary parts D) Quasi normal mode corresponding to the complex eigenfrequency $\omega_{M}$ E) Comparison of the QNM for $\omega_{M}$ (solid line) and the transmission (defect) field (dotted line) in the region around $x=0$ where the incoming field is present. F) Mirror field for the (periodic) structure without defect for $\omega=\operatorname{Re}\left(\omega_{M}\right)$ $G)$ Field associated with the transmission resonance in the defect structure obtained via variational approximation based on mirror field and relevant QNM and compared with TMM reference.

between the approximation obtained form the field template and the exact TMM solution, as can be seen in Fig. 2G).

An acceptable agreement between the approximated field profile and the TMM reference (exact solution) is valid in the whole bandgap frequency range. The field template including an exciting field, together with the variational procedure, provides a constructive quantitative way to relate QNMs and TRMs.

The transmittance (3.22) is compared with the TMM reference calculation, as shown in Fig. 3A). We observe an excellent agreement between the approximation and the TMM calculations. Fig. 3B) shows the frequency dependence of the decomposition coefficient $a_{M}$ for this structure. The resonant response is clearly reflected in this dependence, showing that the transmission resonance is connected with the excitation of the internal dynamics represented by the relevant QNM. 


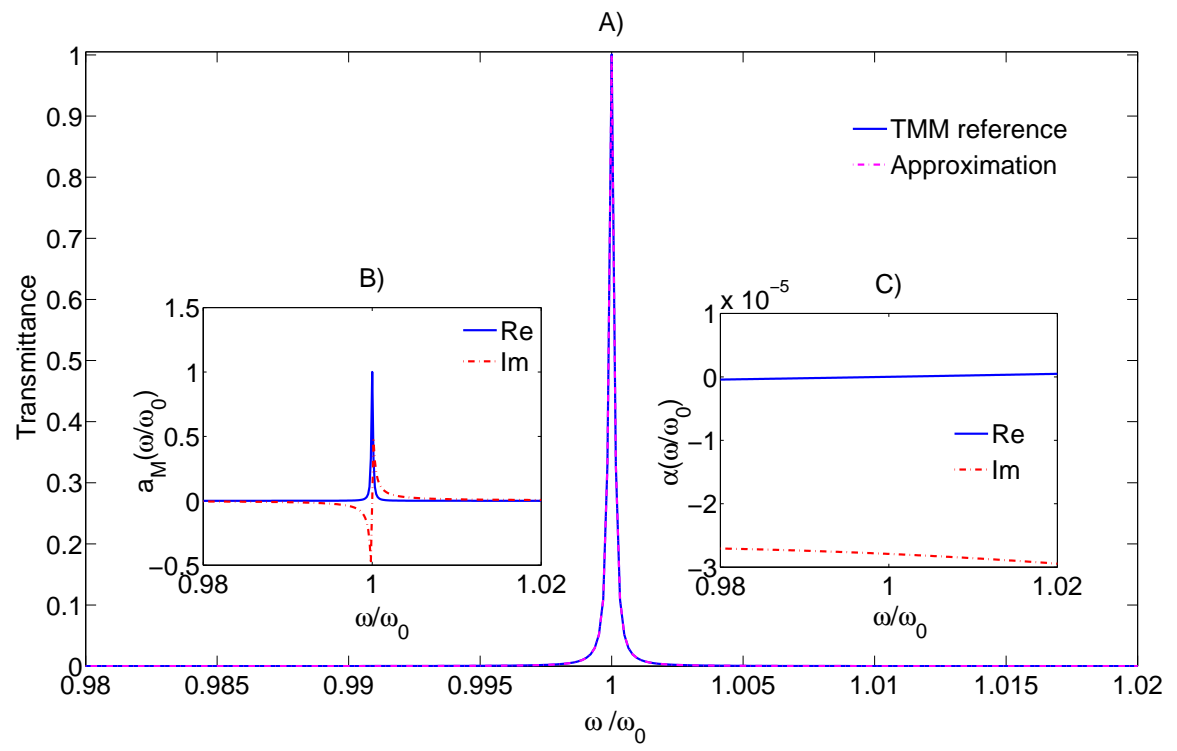

Figure 3.3: A) Transmittance obtained from field representation using QNMs and TMM reference $B$ ) Decomposition coefficients $C$ ) Non-resonant part of the decomposition coefficient.

A common assumption made in the literature is that the spectral transmission for the single resonance situation, as described, is of a Lorentzian lineshape. Our method can analytically justify this assumption. We consider the contribution of a single QNM in the field template (3.16). The equation for the decomposition coefficient then reads

$$
A a(\omega)+b=0 .
$$

After partial integration (3.21) can be given the form

$$
b=\omega^{2} \int_{0}^{L} \frac{n_{0}^{2}(x)-n^{2}(x)}{c^{2}} E_{m f} Q_{q} d x
$$

with $n_{0}$ being the refractive index distribution for the finite periodic (unperturbed) structure. After partial integration, (3.20) reads

$$
A=\left(\omega_{q}^{2}-\omega^{2}\right) \int_{0}^{L} \frac{n^{2}(x)}{c^{2}} Q_{q}^{2} d x+i \frac{\left(\omega_{q}-\omega\right)}{c}\left(n_{\text {in }} Q_{q}^{2}(0)+n_{\text {out }} Q_{q}^{2}(L)\right) .
$$

The amplitude transmission coefficient can be approximated as

$$
t(\omega)=\frac{E_{m f}(L)+a(\omega) Q_{q}(L)}{A_{\text {inc }}} \simeq \frac{a(\omega) Q_{q}(L)}{A_{\text {inc }}}
$$


assuming that the periodic structure represents a "good mirror" with the property $E_{m f}(L) \simeq 0$. The frequency dependence of the transmission amplitude then comes from the decomposition coefficient

$$
a(\omega)=\frac{\alpha(\omega)}{\omega-\omega_{q}} \simeq \frac{\alpha}{\omega-\omega_{q}},
$$

where

$$
\alpha(\omega)=\frac{\omega^{2} \int_{0}^{L} \frac{n_{0}^{2}(x)-n^{2}(x)}{c^{2}} E_{m f} Q_{q} d x}{\left(\omega+\omega_{q}\right) \int_{0}^{L} \frac{n^{2}(x)}{c^{2}} Q_{q}^{2} d x+\frac{i}{c}\left(n_{\text {in }} Q_{q}^{2}(0)+n_{\text {out }} Q_{q}^{2}(L)\right)} .
$$

Term (3.29) is non-resonant in character; in the case of a very narrow resonance $\omega \simeq \operatorname{Re}\left(\omega_{q}\right)$ and $\operatorname{Im}\left(\omega_{q}\right) \ll \operatorname{Re}(\omega)$ can be shown to depend weakly on the frequency, see Fig. 3C). Therefore, in the framework of our approximate model equation (3.28) represents the Lorentzian like shape as shown in Fig 3B). This result agrees with those obtained previously in literature $[65,72,73,26]$ Here it follows from a completely different approach and further supports the conclusion that our method adequately captures the resonance character of the transmission.

\subsubsection{Asymmetric single cavity}

Now the internal structure of the previous example is enclosed within two semiinfinite media of different refractive indices $n_{\text {in }}=1$ and $n_{\text {out }}=5$ (as a somewhat artificial example to emphasize asymmetric nature of the structure). The QNM spectrum and transmittance shown in Fig. 4A) and 4B) and the QNM profile in Fig. 4C) suggest that the difference between the symmetric and asymmetric structures is reflected in the shift of frequency positions in the complex plane.

The same qualitative behavior can be seen as in Fig. 2A) and 2B), i.e. a single resonance appears in the bandgap region when the defect is introduced, now with the lower transmittance level (corresponding to the reflection at an interface between media with indices $n_{\text {in }}$ and $n_{\text {out }}$ [4]). A similar field template as for the symmetric structure is used. This choice is further confirmed by the excellent agreement between the approximation of the transmittance with the TMM reference calculation shown in Fig. 4D).

\subsubsection{Double cavity structure}

For this example, we consider a layer arrangement $(H L)^{4} D(L H)^{2} L D(L H)^{4}$, where two defects are introduced as changes of thicknesses of layers $L_{D}=2 L_{H}$, where $n_{D}=n_{H}$. The refractive index outside the structure is the same on both sides. The values of the refractive indices are the same as in section 3.1. These defects are forming two FP (Fabry-Perot like) resonant cavities enclosed by two identical mirrors and one separating mirror. 

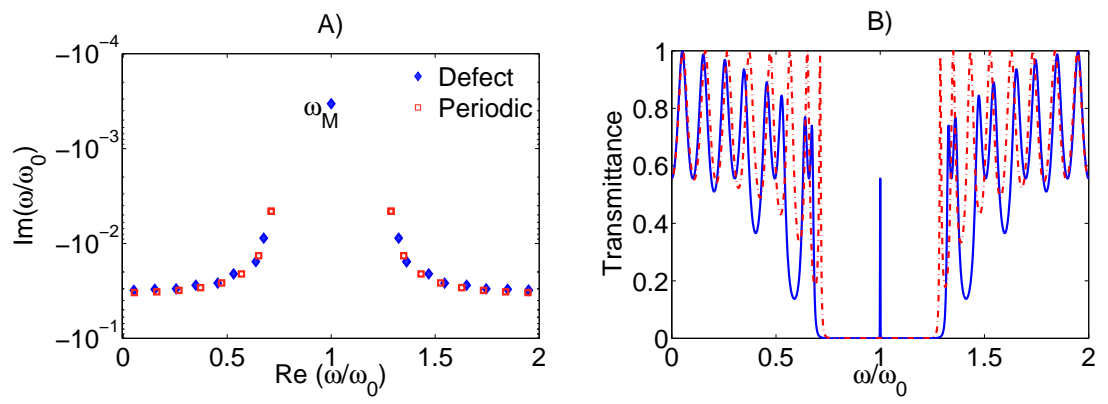

C)
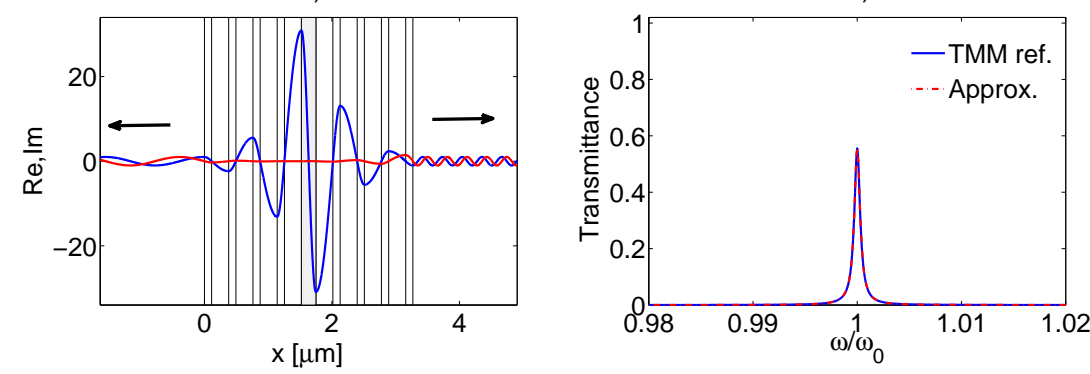

Figure 3.4: A) QNM spectrum and B) Transmittance for the asymmetric periodic (dashed) and defect (continuous) structure C) QNM for the defect structure D) Transmittance obtained from the field representation using QNMs and the TMM reference.

The resonant response of the double-cavity structure is represented by two complex frequencies in the bandgap region as shown in Fig. 5A). The corresponding transmittance plot shows two distinct transmission resonances in the bandgap region Fig. 5B). The QNMs for these two defect-induced eigenfrequencies are shown in Fig 6C) and 6D). Symmetric and skew-symmetric behavior of the eigenfields is present, arising form the overall symmetry of the structure.

Fig. 6A) and 6B) shows the decomposition coefficients and the approximated transmittance response that is in excellent agreement with TMM reference. The field template based on the mirror field of the structure without defects and linear combination of the two relevant QNMs enables an excellent field representation of the transmission resonance modes as can be seen from Figs. 6C) and 6D).

This example can be considered as a case of strongly coupled FP cavities where the interaction is sufficient to introduce a significant separation of the resonance frequencies. This is reflected in the positions of the defect QNM eigenfrequencies of the defect structure. Our approximation method enables both an accurate field representation and predicts the proper resonant transmission. 
A)

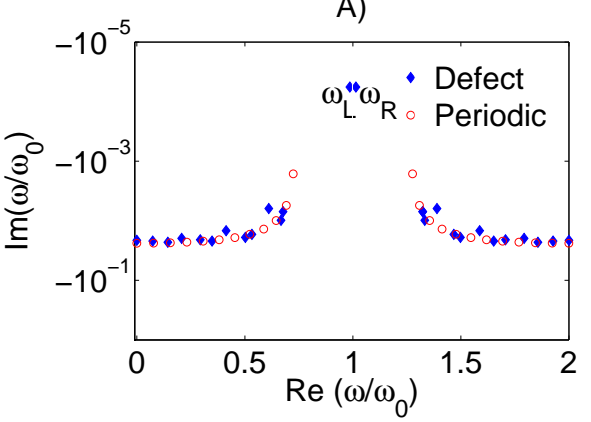

C)

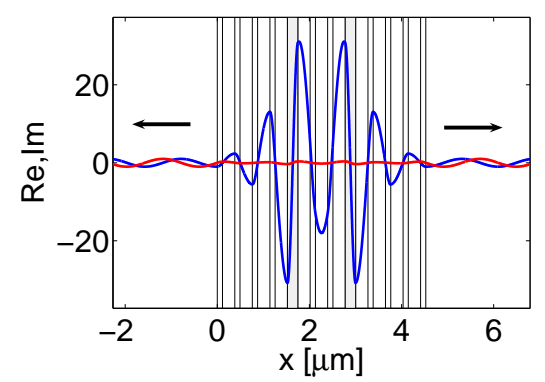

B)

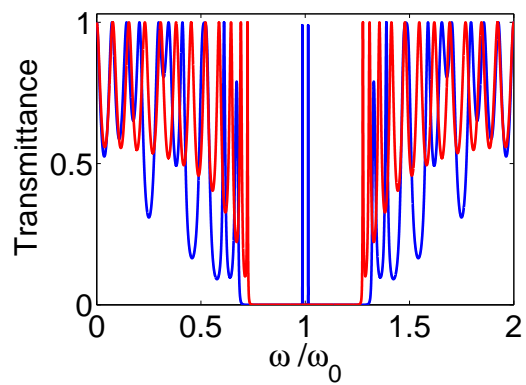

D)

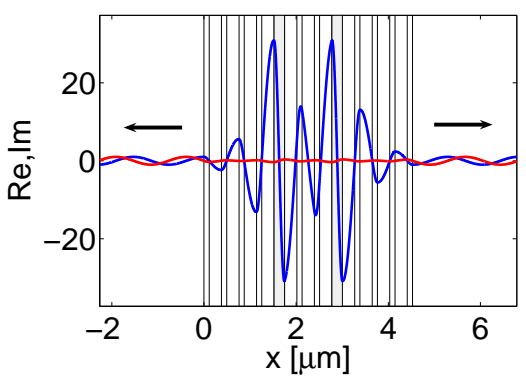

Figure 3.5: A) QNM spectrum B) Transmittance for periodic and double cavity structure; QNMs corresponding to complex frequencies in the bandgap region $C$ ) QNM for $\left.\omega_{L} D\right) Q N M$ for $\omega_{R}$.

\subsubsection{Multiple cavity structure with flat-top narrow-band transmis- sion}

As last example we choose an asymmetric triple cavity structure with layer arrangement coded as $(H L)^{4} L(H L)^{9} L(H L)^{9} L(H L)^{4}, n_{H}=2.1, n_{L}=1.45$, $n_{\text {in }}=n_{\text {out }}=1.52, L_{H}, L_{L}$-quarter-wavelength [6]. This structure introduces three complex eigenfrequencies in the bandgap region, as shown in Fig. 7A). The important feature is that it provides a narrow-band flat-top transmission inside the bandgap region as can be seen in Fig 7B). The closely spaced eigenfrequencies and the corresponding QNMs are shown in Fig. 8. The proximity of the complex frequencies reflects weak coupling between the three individual FP cavities formed by the defects.

Fig. 9 shows the decomposition coefficients and the approximated transmittance (compared with the TMM reference). The close proximity of the eigenfrequencies is reflected in the substantial overlapping of the frequency regions where all three decomposition coefficients contribute. The field pattern in this region is clearly produced by the combination of three relevant QNMs. Obviously all three QNMs play a significant role over the whole transmission band. The approximated field profiles for the transmission pass-band and the immediate bandgap region 
A)

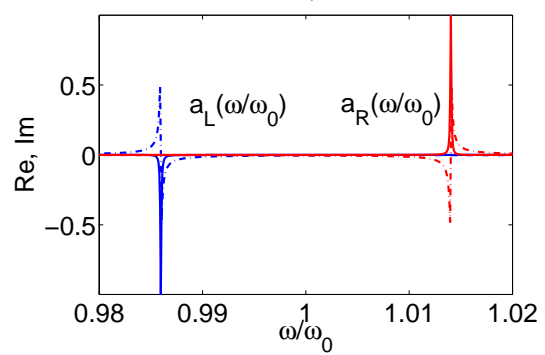

C)

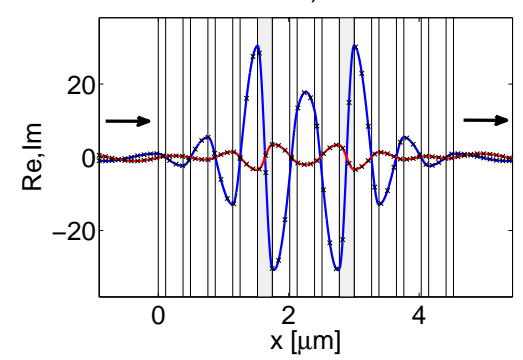

B)

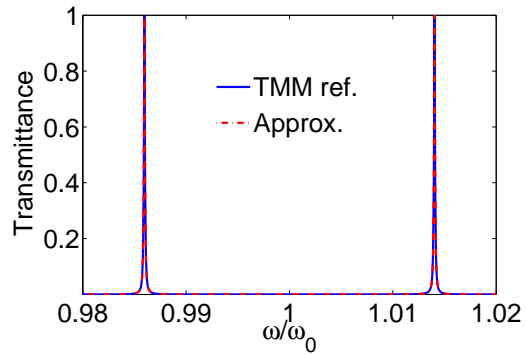

D)

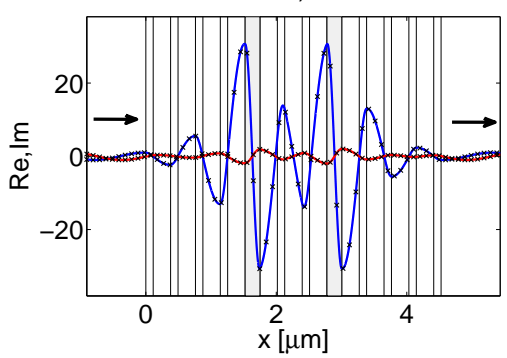

Figure 3.6: A) Decomposition coefficients. B) Transmittance obtained from the field representation using QNMs (dashed) and TMM reference (continuous). C) and D): approximated field obtained from the field representation using QNMs (marker) and TMM reference for the frequency of transmission resonance (solid line) for $\omega=\operatorname{Re}\left(\omega_{L}\right)$ and) $\omega=\operatorname{Re}\left(\omega_{R}\right)$.

agree well with the TMM reference, as shown in Fig. 10.

We wish to point out that direct TMM calculations are not suitable for estimating the resonance origin of the transmission band.Also, some of the methods used in literature, that estimate complex eigenfrequencies by matching the transmittance spectrum to the Lorentzian lineshape functions and estimate eigenfields through association of the TRMs with the QNMs, see [83] and references therein, are not efficient in this case. Neither can estimates of the complex eigenfrequencies and QNMs based on FDTD (finite difference time domain) simulations deal easily with this type of structures with flat-top transmission, see [7] and references therein.

In contrast our model permits to observe directly the relevance of the individual QNMs at different frequencies (i.e. the magnitude of the expansion coefficients $\left.a_{p}\right)$.

\subsection{Conclusions}

We proposed a constructive way of connecting a quasi-normal mode (eigenmode) description with transmission resonance properties for optical defect microcavities 

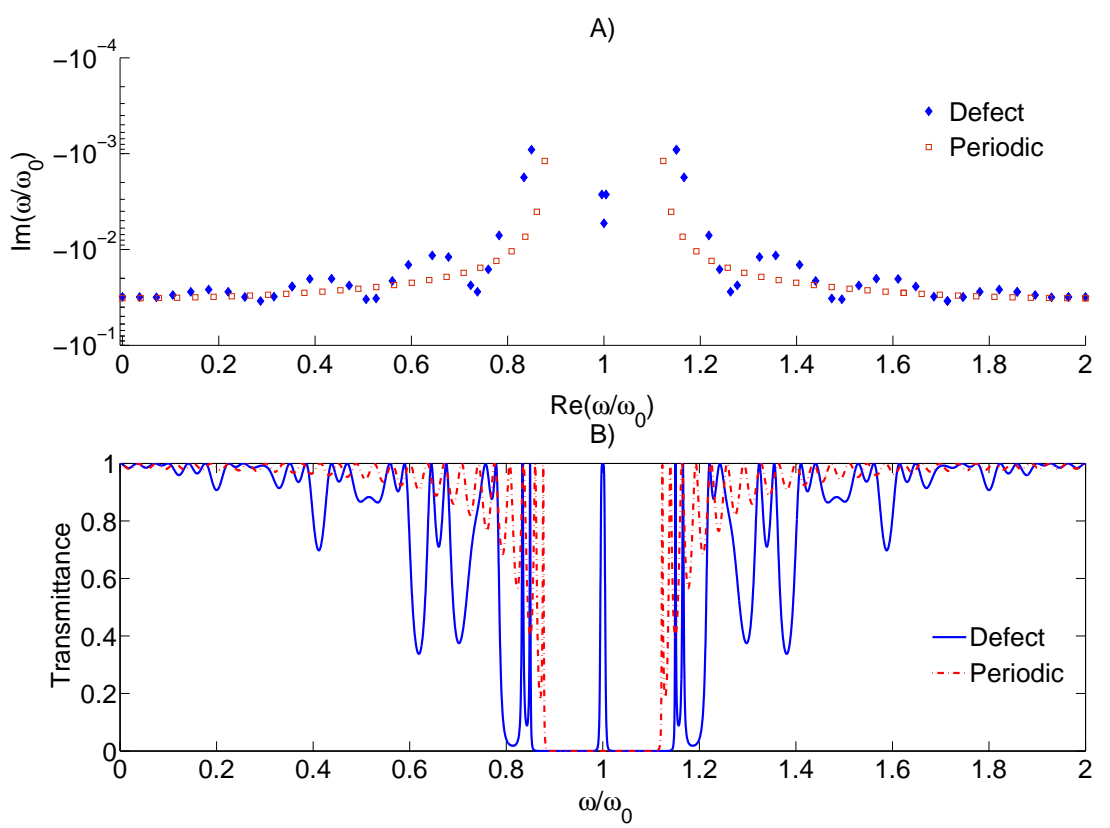

Figure 3.7: A) QNM eigenfrequencies for the triple-defect structure and periodic (unperturbed) structure, and B) transmittance.

in 1D multilayer structures. The approach is meant specifically for approximations of the defect induced transmission modes existing in the bandgap of otherwise periodical structures.

The field representation using a mirror field and the most relevant QNMs enables very accurate field representations for field profiles in the transmittance problem. The approximated spectral transmittance agrees excellently with the TMM reference. We emphasize the open and finite nature of the structures by directly characterizing resonance properties via an investigation of the quasi-normal mode spectrum.

Numerical examples suggest that the method is valid for single and multiple cavity structures in both symmetric and nonsymmetric layer arrangements and both weak and strong couplings between defects. Moreover, our method allows to examine directly the resonance nature of the transmission response in cases where it is very hard to establish this from exact solutions of the transmission problem, such as provided by the TMM method.

The approach quantifies directly the physical viewpoint, where the defect cavities are regarded as externally forced oscillators. The field representations obtained using QNMs have a better foundation in the physics and nature of the realistic, finite structures, when compared with methods that assumes periodic boundary conditions for the structure.

We believe that our approach can be generalized to 2D and 3D structures as an 


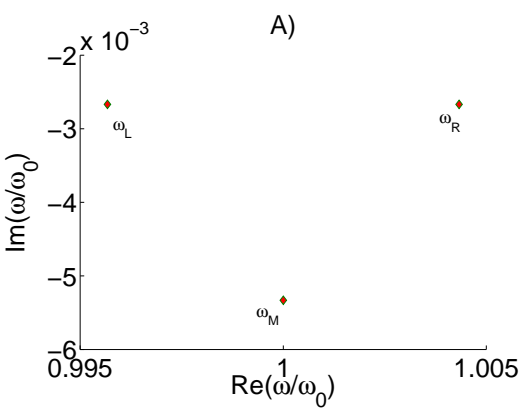

C)

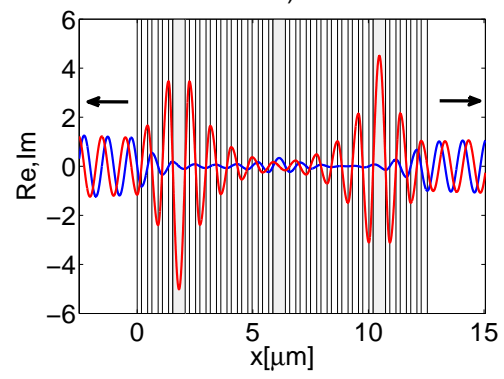

B)

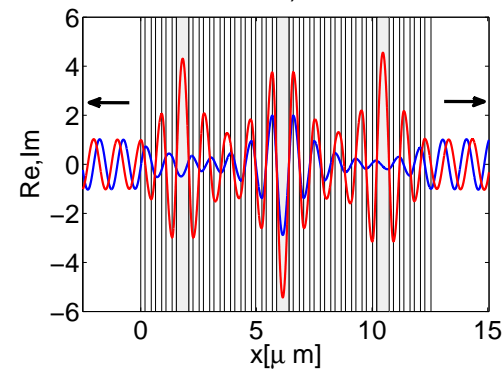

D)

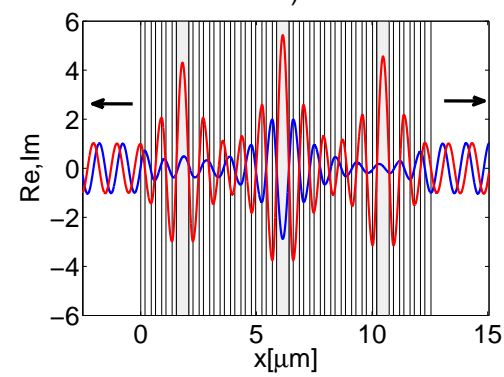

Figure 3.8: A) Complex eigenfrequencies of defects induced QNMs in the triplecavity structure, QNMs for $\left.\omega_{L} B\right) \omega_{M} C$ ) and $\left.\omega_{R} D\right)$.
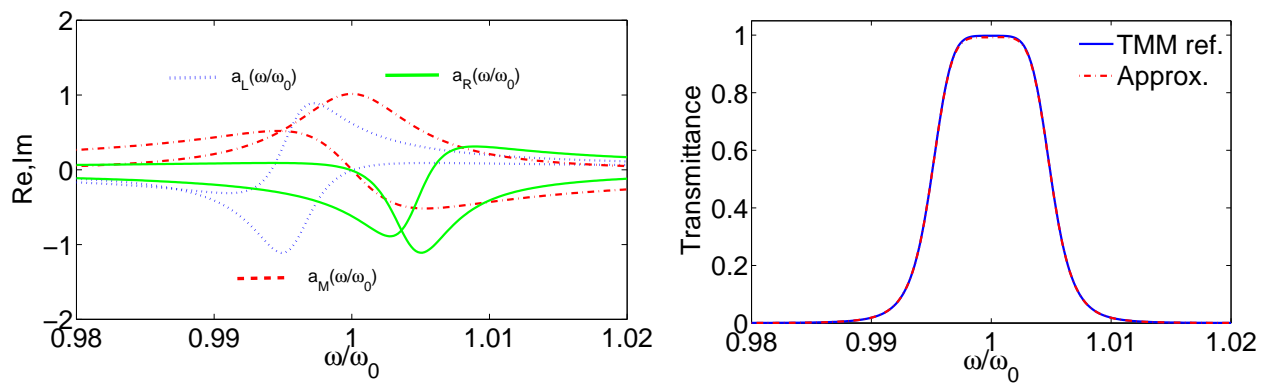

Figure 3.9: Decomposition coefficients for the field representation, corresponding to the QNMs associated with $\omega_{L}, \omega_{M}, \omega_{R}$ (left) and the transmittance obtained from the field representation using QNMs and TMM reference (right).

acceptable characterization for both fields and response functions. Provided that suitable QNM basis fields can be made available by analytical or numerical means, generalizations could be based on the functional representations of the frequency domain Maxwell equations for higher dimensions [84],[85]. 
A)

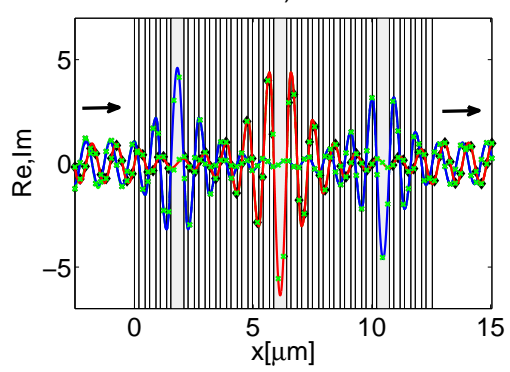

C)

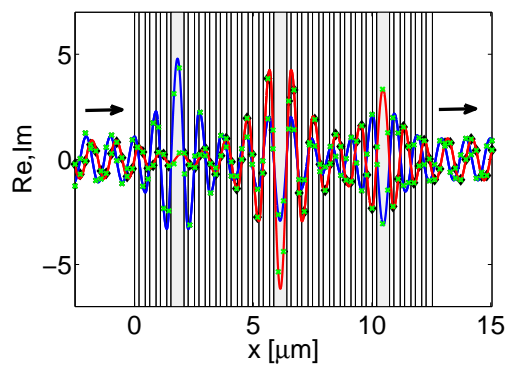

B)

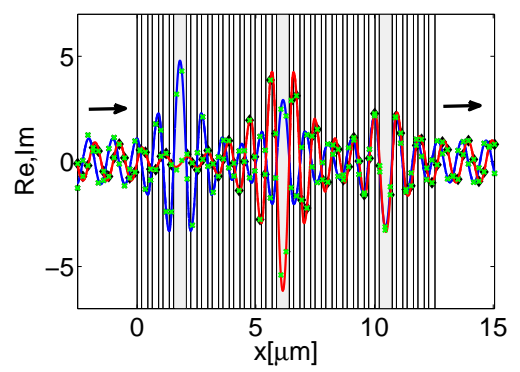

D)

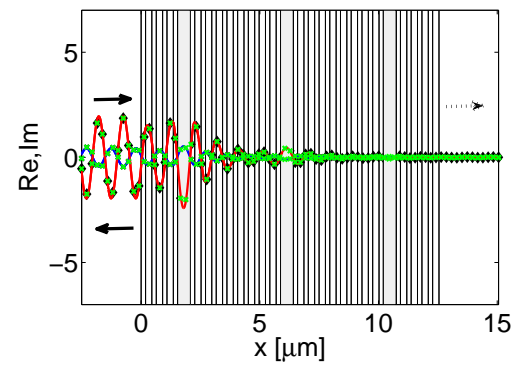

Figure 3.10: Field profiles for transmission in and out of the pass-band obtained from the field representation using QNMs (marker) and compared to the TMM reference (solid line) A) $\omega / \omega_{0}=1 B$ ) $\omega / \omega_{0}=1.002$ C) $\omega / \omega_{0}=0.998 \mathrm{D}$ ) $\omega / \omega_{0}=0.98$. 


\title{
Chapter 4
}

\section{Coupled optical defect microcavities in 1D photonic crystals and quasi-normal modes}

\begin{abstract}
$^{1}$
We analyze coupled optical defect cavities realized in finite one-dimensional Photonic Crystals. Viewing these as open systems where waves are permitted to leave the structures, one obtains eigenvalue problems for complex frequencies (eigenvalues) and Quasi-Normal-Modes (eigenfunctions). Single defect structures (photonic crystal atoms) can be viewed as elementary building blocks for multipledefect structures (photonic crystal molecules) with more complex functionality. The QNM description links the resonant behavior of individual PC atoms to the properties of the PC molecules via eigenfrequency splitting. A variational principle for QNMs permits to predict the eigenfield and the complex eigenvalues in $P C$ molecules starting with a field template incorporating the relevant QNMs of the PC atoms. Both the field representation and the resonant spectral transmission close to these resonances are obtained from a variational formulation of the transmittance problem using a template with the most relevant QNMs. The method applies to both symmetric and nonsymmetric single and multiple cavity structures with weak or strong coupling between the defects.
\end{abstract}

\footnotetext{
${ }^{1}$ This chapter is adapted from: M. Maksimovic, M. Hammer, E. van Groesen, Coupled optical defect microcavities in $1 D$ photonic crystals and quasi-normal modes, Photonics West 2008 / OPTO 2008, Integrated Optics: Devices, Materials, and Technologies XII, Proceedings of SPIE,Vol. 6896, 2008
} 


\subsection{Introduction}

Photonic Crystal (PC) based devices attracted much interest in the past two decades concerning both fundamental and applied aspects. Plenty of modeling and computational techniques are applied and well established [7, 9, 6]. We consider 1-D PC structures that can provide qualitative insight and means for interpreting the physics of higher dimensional structures. More specifically, we consider planar layered inhomogeneous media with piecewise constant refractive index as the traditional model of 1-D PCs. Although they belong to the field of multilayer optics [4], an old and well explored field, a novel way of modeling these devices has certain theoretical and practical interest for itself.

The open and finite nature of realistic structures is accessible by directly characterizing resonance properties via an investigation of the quasi-normal modes and associated complex frequencies. Quasi-normal modes (QNMs) are eigenfunctions associated with the complex eigenfrequencies arising from the eigenvalue problem for outgoing waves [24]. The real parts of the complex eigenfrequencies are connected with the transmission resonance frequencies (local maxima of the transmission) and the imaginary parts with the Q-factors (or linewidth) of the resonant transmission profile. Properties of the QNMs and related PC structures have been addressed for 1-D PC structures in [65, 72, 77], while for 2-D PC structures the theory is by far less often addressed and developed, with only partial results [81].

We specialize to finite PC structures with suitable defects in otherwise periodic arrangements. These defects are forming Fabry-Perot cavities enclosed by and separated by leaky mirrors that allow the exchange of energy between cavities. These Coupled Optical Microcavites (CMC) already attracted research interest as they provide means for the implementation of optical filters, resonators, delay lines and other devices in both passive and active structures [6, 86, 87, 88, 89]. Reference method for analyzing one-dimensional structures is a Transfer Matrix Method (TMM) [4]. A description in the framework of different coupled mode theory approaches has been a traditional way of analysis [84, 90, 91, 92], as far as interacting optical waveguides (i.e., mostly systems with well confined optical states) are concerned. However, an analysis of open, leaky structures directly based on QNMs seems to be missing. This paper considers some possibilities for the direct characterization of open cavities in 1-D PC structures using only the most relevant QNMs.

Composite CMC structures can be viewed as being formed from simpler single cavity structures or some other elementary building blocks. This decomposition is usually quite arbitrary and can be done in many different ways for a given structure. However, when the individual modes are well localized in the vicinity of their respective cavities, a field template for the composite structure can be based on the superposition of the individual cavity modes. In literature the basic structures are sometimes called "photonic crystal atoms" which are the elementary building blocks for more complex "photonic crystal molecules". The key idea is that by combining PC atoms with known properties more complex PC molecules can be 
obtained with engineered properties. Based on QNMs and a variational principle, our procedure enables the derivation of the properties of the composite structures in a constructive way using the known properties of the building blocks and certain design rules for the composite structure.

In the context of CMCs, we address the splitting of eigenfrequencies by using a variational principle together with the related QNMs of the individual cavities. QNMs of the composite structure (super-modes) can be approximated by this approach. Further, we use the characterization of the CMCs in terms of quasi-normal modes to describe approximately the resonant response to an external excitation in the frequency domain and the related field profiles. The approximate frequency domain description follows from a suitable variational formulation [25] for the transmission problem, using the most relevant QNMs in establishing appropriate field templates [93].

\subsection{Theory}

We consider 1-D optical structures in the frequency domain under external excitation. The optical field $E(x)$ excited by the external influx $E_{i n c}=A_{i n c} e^{i\left(n_{i n} \omega / c\right) x}$, with $\omega \in \mathbb{R}$ and $A_{\text {inc }}$ given, for vacuum speed of light $c$, satisfies the Helmholtz equation

$$
\partial_{x}^{2} E+\frac{\omega^{2}}{c^{2}} n^{2}(x) E=0,
$$

on an interval $x \in[L, R]$, and transparent influx boundary conditions

$$
\left(\partial_{x} E+i \frac{\omega}{c} n_{\text {in }} E\right)_{x=L}=2 i \frac{\omega}{c} n_{\text {in }} A_{\text {inc }}, \quad\left(\partial_{x} E-i \frac{\omega}{c} n_{\text {out }} E\right)_{x=R}=0
$$

at the boundaries $x=L, R$. The exterior regions $x<L$ and $x>R$ are assumed to be homogeneous with refractive indices $n_{\text {in }}$ and $n_{\text {out }}$, respectively. For structures with piecewise constant refractive index an exact solution can be obtained via a standard and well known transfer matrix method [4]; a brief explanation is given in appendix 4.5. This serves as reference for the approximate models discussed below.

Properties of passive, open optical structures with energy exchange between the constitutive elements and the environment are captured adequately by a formulation of an eigenvalue problem for complex frequencies. A finite structure can be viewed as an open system with transparent boundaries which permit the leakage of energy to the exterior, see Figure 4.1 A). The electric field in the interior $x \in(L, R)$ satisfies the Helmholtz equation:

$$
\partial_{x}^{2} Q+\frac{\omega^{2}}{c^{2}} n^{2}(x) Q=0
$$

with outgoing wave boundary conditions

$$
\left(\partial_{x} Q+i \frac{\omega}{c} n_{i n} Q\right)_{x=L}=0, \text { and }\left(\partial_{x} Q-i \frac{\omega}{c} n_{\text {out }} Q\right)_{x=R}=0 .
$$


This constitutes an eigenvalue problem for the frequency $\omega$ as the complex eigenvalue and the field profile $Q(x)$ as eigenfunction (Quasi-Normal Mode) [24, 26, $65,72]$. The eigenvalue problem is nonlinear because the eigenvalue appears in the boundary conditions explicitly [26]. QNMs can be used to solve the initialvalue problem of energy leakage out of a given open structure. The applicability of QNMs for solutions of the transmission problems with given influx relies on specific pseudo-orthogonality and completeness properties of QNMs when used as a basis set for an eigenfunction expansion $[65,72]$.

A variational formulation of the QNM eigenvalue problem can be based on the functional [25]

$$
\begin{aligned}
\mathcal{L}_{\omega}(Q) & =\frac{1}{2} \int_{L}^{R}\left(\left(\partial_{x} Q\right)^{2}-\frac{\omega^{2}}{c^{2}} n^{2}(x) Q^{2}\right) d x \\
& -\frac{i \omega}{2 c}\left(\left.n_{\text {in }} Q^{2}\right|_{x=L}+\left.n_{\text {out }} Q^{2}\right|_{x=R}\right) .
\end{aligned}
$$

If $\mathcal{L}_{\omega}$ becomes stationary, i.e. if the first variation of $\mathcal{L}_{\omega}(Q)$ vanishes for arbitrary variations of $Q$, then $Q$ satisfies equation (4.3) with equations (4.4) as natural boundary conditions. The value of the functional (4.5) with the proper eigenfunction/ eigenvalue pair $(\omega, Q)$ inserted is zero, i.e.

$$
\mathcal{L}_{\omega}(Q)=0
$$

This property can be shown analytically by computing the partial derivative of the first term in the (4.5).

We specialize to the analysis of optical defect modes existing in the bandgap of the underlying periodic structure. To avoid using the full set of QNMs and the completeness properties of QNMs to determine approximations of the optical transmission and of the related field profiles, we apply a variational principle and a specific field template that consists of QNMs associated only with the optical defects. Details of this procedure can be found in [93] and in appendix 4.6.

\subsubsection{Coupled cavities}

We start with the QNMs $\left(\omega_{1}, Q_{1}\right), \ldots,\left(\omega_{N}, Q_{N}\right)$ for refractive index distributions $n_{1}(x), \ldots, n_{N}(x)$ of simpler (not necessarily single cavity) structures. Solutions of the eigenvalue problem for the composite structure are assumed to be well approximated by linear combinations of the QNMs belonging to the simpler structures. Therefore, we choose the field template

$$
Q=\sum_{p=1}^{N} a_{p} Q_{p}
$$

which represents a restriction $\mathcal{L}_{\omega}(Q) \rightarrow L_{\omega}\left(a_{1}, \ldots, a_{N}\right)$ of the solutions of the original problem. Stationarity of the functional (4.5) transforms on the restricted 


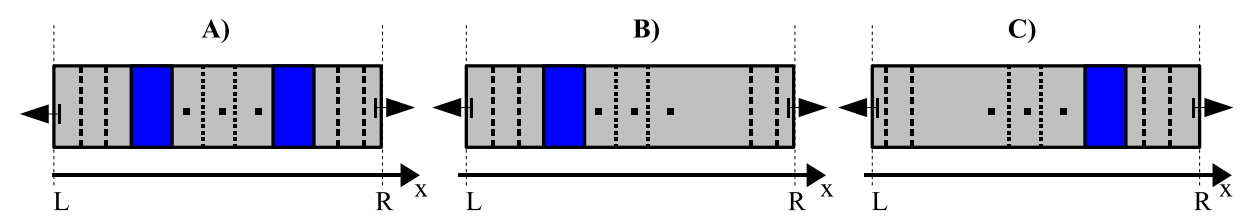

Figure 4.1: The coupled optical defect structures considered in this paper are finite periodic multilayer structures consisting of two materials with high index $n_{H}$ and low index $n_{L}$. The layer thicknesses $d_{H}, d_{L}$ are chosen to be quarter-wavelength for the target wavelength (related to a reference frequency $\omega_{0}$ ). Optical defects are introduced as changes of the layer thicknesses or refractive indices in the otherwise periodic sequence. The whole structure is enclosed by two semi-infinite media of indices $n_{\text {in }}$ and $n_{\text {out }}$. A composite multiple defect structure A) can be decomposed into usually simpler single defect structures B) and C).

set to the conditions

$$
\frac{\partial L_{\omega}}{\partial a_{p}}\left(a_{1}, \ldots, a_{p}, \ldots, a_{N}\right)=0, \text { for } p=1, \ldots, N,
$$

that can be written as an algebraic quadratic eigenvalue problem [94]

$$
\left(\omega^{2} \mathbf{M}+\omega \mathbf{N}+\mathbf{P}\right) \mathbf{a}=0
$$

for the complex eigenfrequencies $\omega$ of the composite system. The eigenvectors $\mathbf{a}=\left[a_{1}, \ldots, a_{N}\right]^{T}$ are the unknown coefficients in the linear superposition (4.7) of the single cavity QNMs. The elements of the matrices $\mathbf{M}=\left[M_{l k}\right]_{N \times N}, \mathbf{N}=$ $\left[N_{l k}\right]_{N \times N}, \mathbf{P}=\left[P_{l k}\right]_{N \times N}$ are

$$
\begin{aligned}
M_{l k} & =-\frac{1}{c^{2}} \int_{L}^{R} n^{2}(x) Q_{l} Q_{k} d x, \\
N_{l k} & =-\frac{i}{c}\left(\left.n_{\text {in }} Q_{l} Q_{k}\right|_{x=L}+\left.n_{\text {out }} Q_{l} Q_{k}\right|_{x=R}\right), \\
P_{l k} & =\int_{L}^{R} \partial_{x} Q_{l} \partial_{x} Q_{k} d x .
\end{aligned}
$$

Equation (4.9) enables the approximate solution of the eigenvalue problem for the composite structure. It directly links the resonance behavior of the individual constitutive elements ( $\mathrm{PC}$ atoms) to the resonance properties of more complex structures (PC molecules), i.e. describes the eigenfrequency splitting. Both resonant frequencies and the related Q-factors can be estimated. Influences of the external and internal confinement (type, length and strength of the "mirrors" in the structure) or perturbations of various parameters can be directly analyzed.

Usually the decompositions of the composite structure, i.e. the precise choice of the elements $Q_{p}$ in (4.7) is to some degree arbitrary. Supporting arguments can 
be based on results from direct computations, on physical intuition, but also on the following observation. For fields $Q_{l}$ with associated frequency $\omega_{l}$ and refractive index $n_{l}$ that satisfy (4.3), (4.4), equation (4.9) can be written as

$$
\mathbf{S} \mathbf{a}=0,
$$

where

$$
\begin{aligned}
S_{l k} & =\int_{L}^{R} \frac{\omega^{2} n^{2}(x)-\omega_{l}^{2} n_{l}^{2}(x)}{c^{2}} Q_{l} Q_{k} d x \\
& +\frac{i\left(\omega-\omega_{l}\right)}{c}\left(\left.n_{\text {in }} Q_{l} Q_{k}\right|_{x=L}+\left.n_{\text {out }} Q_{l} Q_{k}\right|_{x=R}\right) .
\end{aligned}
$$

If the trial field includes the exact solution for the composite structure with the property $\omega=\omega_{l}$ then (4.11) is satisfied. Expression (4.12) suggests that the refractive index distributions $n_{l}$ of the simpler structures in the decomposition should be chosen as close as possible to the exact structure (refractive index $n$ ).

\subsubsection{First order perturbation correction for complex eigenfrequen- cies}

We look for corrections of the complex eigenfrequencies for a given structure when small, localized perturbations of the permittivity are present. A first order perturbation correction for the complex eigenvalue can be obtained by using (4.5) and a known QNM eigenpair $\left(\omega_{0}, Q_{0}\right)$ of the unperturbed problem with refractive index distribution $n_{0}(x)$. It is reasonable to assume that a small perturbation of the original structure does neither change substantiality the position of the complex eigenfrequencies in the complex plane nor the shape of the corresponding QNMs. We consider a permittivity perturbation in the form

$$
n^{2}(x)=n_{0}^{2}(x)+n_{p}^{2}(x) .
$$

For small (in effect) perturbations $n_{p}^{2}$ we look for a first order correction $\omega_{1}$ to the eigenfrequency $\omega=\omega_{0}+\omega_{1}$. Variational accuracy guarantees that the eigenfrequency is determined up to first order if the eigenfunction is known up to zeroth order (solution of the unperturbed structure). Upon restricting (4.5) to the zeroth order field approximation $\mathcal{L}_{\omega}\left(a Q_{0}\right) \rightarrow L(a)$, the stationarity condition on the restricted set

$$
\frac{\partial L}{\partial a}(a)=0
$$

gives an equation for the eigenfrequency correction. Keeping only the first order terms in $\omega_{1}$ and using the property (4.6) satisfied by the eigenpair $\left(\omega_{0}, Q_{0}\right)$ of the unperturbed problem, the correction to the complex eigenfrequency reads

$$
\omega_{1}=-\frac{\omega_{0}^{2}}{c^{2}} \frac{\int_{L}^{R} n_{p}^{2}(x) Q_{0}^{2} d x}{2 \frac{\omega_{0}}{c^{2}} \int_{L}^{R} n_{0}^{2}(x) Q_{0}^{2} d x+\frac{i}{c}\left(\left.n_{\text {in }} Q_{0}^{2}\right|_{x=L}+\left.n_{\text {out }} Q_{0}^{2}\right|_{x=R}\right)} .
$$


Obviously this procedure is closely related to the theory of (4.2.1); it may be viewed as a "coupled mode theory" with only one mode in the template (4.7). It is possible to extend this method and to derive both corrections to the eigenvalue and to the eigenfunction up to arbitrary order using a variational principle. An iterative procedure for higher order corrections will be reported elsewhere.

\subsection{Results and Discussion}

A series of examples of CMCs serves to validate the described methods. First, we apply the variational principle of Section 4.2.1 for approximating supermodes in a double-cavity structure using known QNMs of the individual single cavities. Second, the variational form of first order perturbation theory for QNMs (Section 4.2.2) is used to analyze shifts of cavity resonances subjected to local perturbations of the refractive index. Third, the method of appendix 4.6 is applied to estimate the transmission on the basis of a few, most relevant QNMs. Finally, we consider multiple-defect structures designed to operate in weak and in strong coupling regimes. Also here our variational approximation method links the resonant transmission to the underlying QNMs.

\subsubsection{Double cavity structure}

Consider a layer arrangement coded as $(H L)^{M_{1}} D(L H)^{M_{1}}$, where $M_{1}=4$ is the number of layer pairs in two mirrors that enclose a single cavity, with $n_{H}=3.42$, $n_{L}=1.0$, between two semi-infinite media of the same refractive index $n_{\text {in }}=$ $n_{\text {out }}=1.0$. The defect is introduced as a central layer of thickness $d_{D}=2 d_{H}$ with high refractive index $n_{D}=n_{H}$. A complex QNM eigenfrequency associated with the defect is present in the bandgap region of the related periodic structure. This eigenfrequency has an imaginary part that is several orders of magnitude smaller (absolute value) than all other eigenfrequencies in the QNM spectrum [93]. Usually this is a sign of a strong localization of the field, i.e. for efficient energy trapping in the vicinity of the defect.

The combination $(H L)^{M_{1}} D(L H)^{M_{2}} L D(L H)^{M_{1}}$ of two of these single cavity structures constitutes a multilayer arrangement with two defects and three mirrors (two enclosing mirrors of "length" $M_{1}$, one separating mirror of length $M_{2}$ ). The defects form two Fabry-Perot-like resonant cavities with two corresponding QNMs and eigenfrequencies, see Figure 4.2 A). These eigenfrequencies correspond to two transmission resonances (Figure 4.2 B) ). The resonant response of the doublecavity structure (the PC molecule) can be viewed as being generated through eigenfrequency splitting from the resonance of the single cavities (the PC atoms). By changing the number $M_{2}$ of pairs in the separating mirror one can control the interaction strength between the two cavities, where the relative distance of the complex frequencies reflects weak or strong coupling. If the separation is small, the over-

lap of the individual QNMs is substantial, which results in a strong separation of 
eigenfrequencies. Increasing the separation leads to close eigenfrequencies and results in the formation of a transmission pass-band. With a field template (4.7) that consists of a linear superposition of the two QNMs associated with the individual left and right cavities, the procedure of Section 4.2.1 permits the estimation of both eigenfrequencies and QNMs of the PC molecule. According to Figure 4.2 this is an excellent approximation even for quite moderate cavity separations $M_{2}$ with rather strong interaction.

In contrast to the composite structure, the permittivity profiles that constitute the PC atoms do not show a particular symmetry (cf. Figure 4.1). Hence the QNMs associated with the individual cavities do not exhibit a special symmetry. When the decomposition is performed properly, however, their symmetric and skewsymmetric linear combinations approximate the symmetric and skew-symmetric supermodes of the composite structure, see Figure 4.2 C) and D).
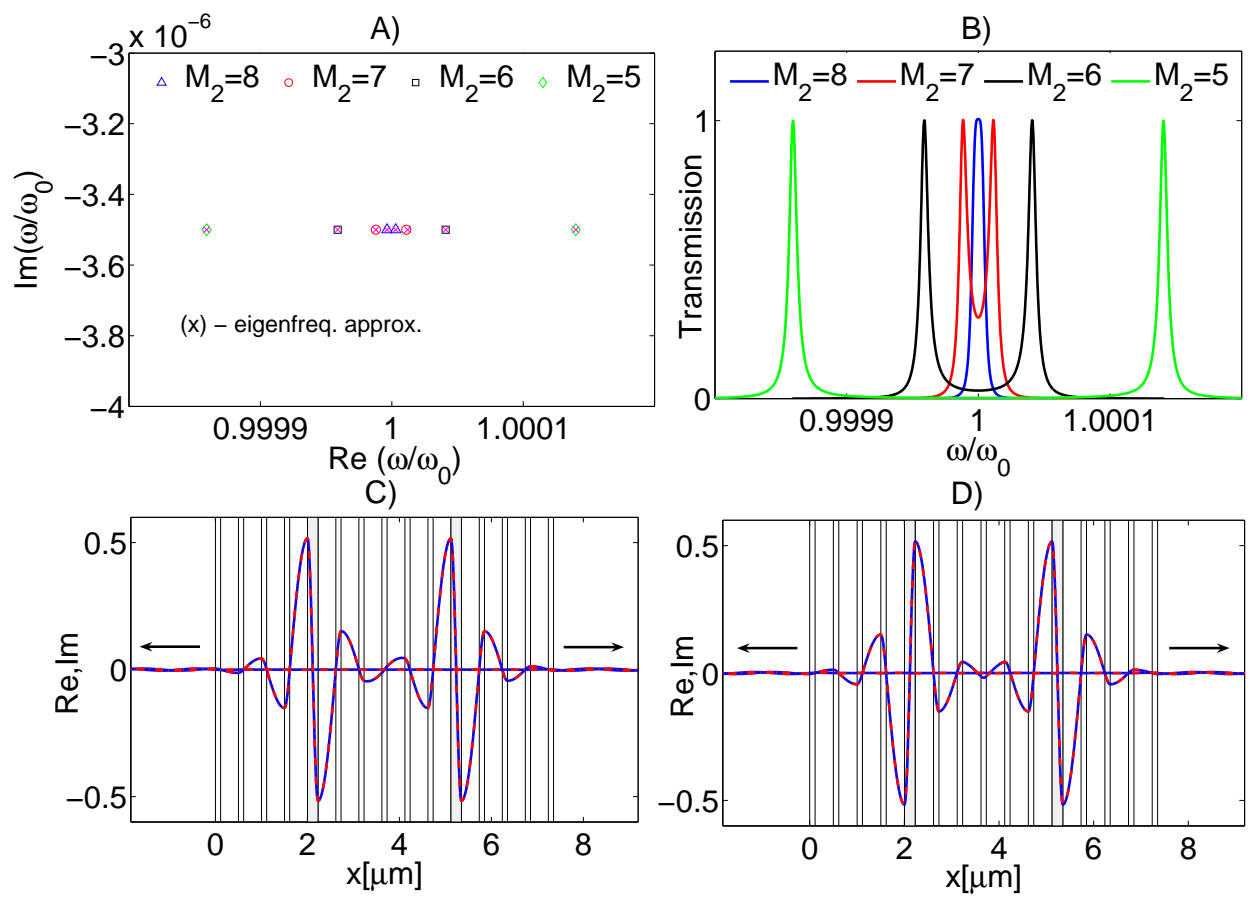

Figure 4.2: A): complex eigenfrequencies $\omega$ for the double cavity structure, direct computations and CMT approximations for different lengths $M_{2}$ of the separation region; B): transmittance, direct TMM calculation; C), D): QNMs (supermodes) for the double cavity structure with $M_{2}=5$, direct computation (continuous) and CMT approximation (dashed).

Further, the variational method of appendix 4.6 allows to characterize the contributions of individual QNMs to the spectral transmission. Figure 4.3 compares two different settings: The template (4.21) for the transmission field can be based either on the two (exact) supermodes of the PC molecule, or on the QNMs sup- 

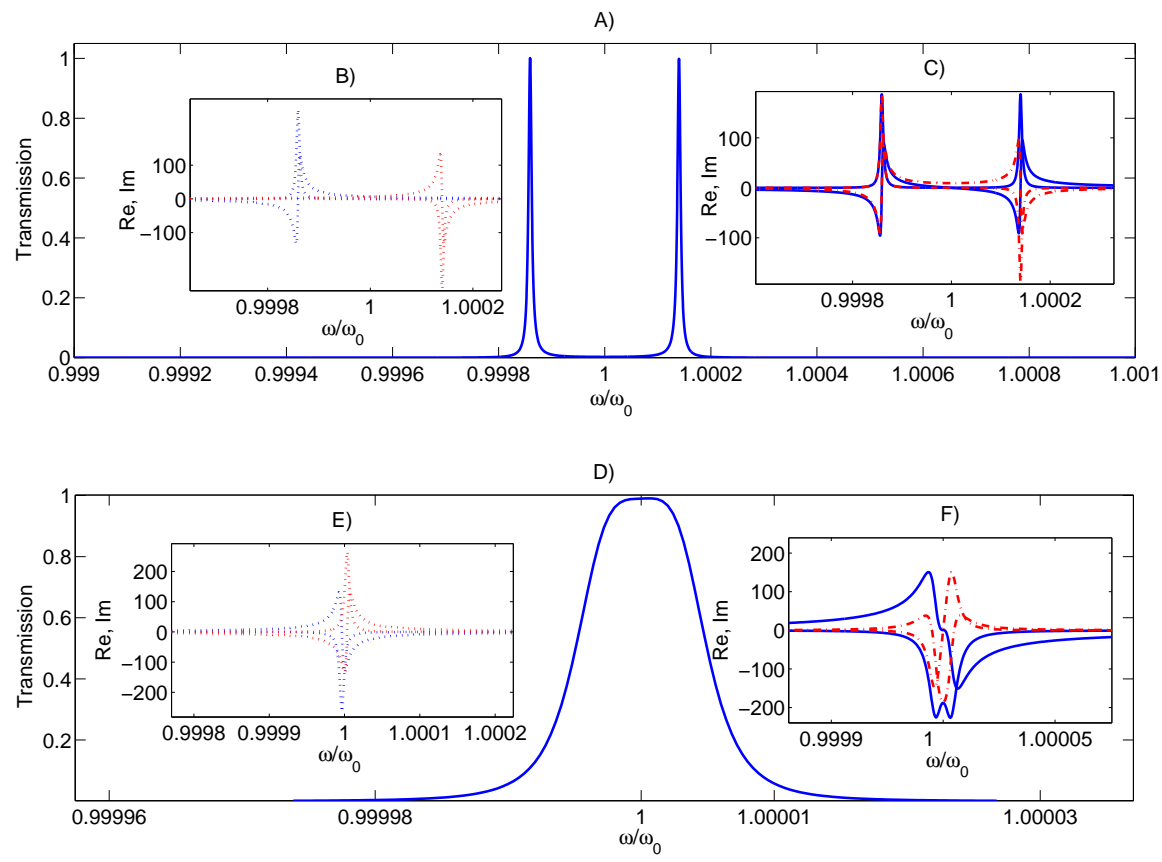

Figure 4.3: Transmittance (large axes, A), D); direct TMM computations and CMT model, superimposed curves) and coefficients $a_{p}$ for approximations (appendix 4.6) to the transmission problem, if the template (4.21) includes either the two exact supermodes of the composite structure (left insets B), E)) or alternatively the QNMs associated with the individual left and right cavities (right insets $C$ ), F)). The upper plots $A$ ), B), C) correspond to a moderate cavity separation $M_{2}=5$, the lower plots $D), E), F$ ) to a setting with $M_{2}=8$, i.e. with weaker interaction.

ported by the PC atoms. In both cases the resulting approximations for the transmission are indistinguishable (on the scale of the figure) from the TMM reference. Especially interesting is the weak coupling regime, where the direct computation based on the TMM method can not easily explain the resonant character of the transmission band. However, examination of the relevant complex eigenvalues, of the QNMs, and of the expansion coefficients describes completely the resonant character of the transmission band.

\section{Perturbation of the double cavity structure}

The perturbation theory from Section 4.2.2 is applied to analyze eigenfrequency shifts due to small local perturbations of the cavity refractive index. Below we look at both symmetric and asymmetric perturbations of the symmetric original structure. The perturbative correction for QNMs estimates reasonably, in first order, both real and imaginary parts of the complex eigenfrequencies. This can be traced 
further to changes of the transmission, i.e. to the position of resonance frequencies and the related Q-factors. Figure 4.4 introduces the specific configuration.

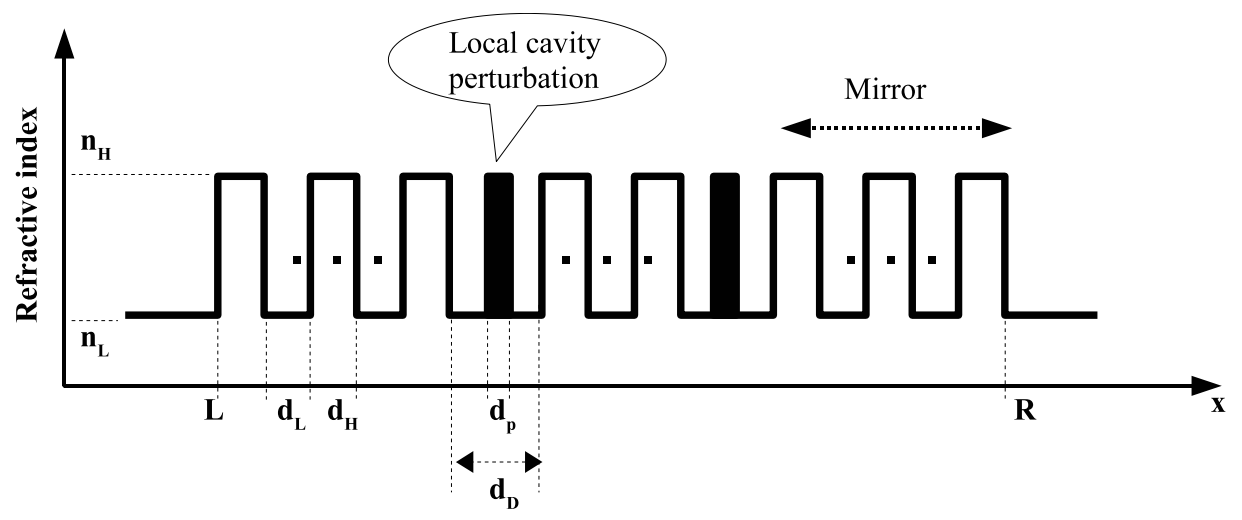

Figure 4.4: Perturbation of a CMC by localized refractive index changes. The double cavity structure is encoded as $(H L)^{4} H(2 L)(H L)^{8} H(2 L)(H L)^{4} H$. The individual layers with alternately high $(H)$ and low refractive index $(L)$ are quarterwavelength with $n_{H}=1.5, n_{L}=1, n_{\text {in }}=n_{\text {out }}=1$. Two low index layers with larger half-wavelength thickness $d_{D}$ form the two defects. Perturbations are introduced as local changes of the permittivity $n^{2}=n_{L}^{2}(1+p)$ in the middle of the defect layer with a thickness of $d_{p}=d_{D} / 5$ and $p \in(0,0.05)$.

First we consider an asymmetric perturbation, where the refractive index of only one of the defects is raised locally. According to Figure 4.5, this leads not only to shifts in the positions of the eigenfrequencies (A)), but also to dramatic changes of the transmission response (B)). The perturbation corrections (4.15) are obtained here with the QNMs (supermodes) of the original composite structure. Figure $4.5 \mathrm{~A}$ ) shows the paths of the eigenfrequencies in the complex plane for varying strength $p$ of the perturbation, where the influence of the refractive index change has been evaluated by expression (4.15) on the one hand, and, for comparison, by direct TMM calculations on the other hand. As expected, the straight lines given by the first order perturbational expression are tangential to the reference paths. In this case the range of a reasonable approximation level is rather limited, because the perturbation destroys the overall symmetry of the structure.

If, in contrast, both cavities are perturbed in a symmetrical way, the results of the perturbational procedure are accurate over a much larger range of perturbation strengths, as seen in Figure 4.5 C). Now the eigenfunctions of the perturbed structured retain their symmetry, i.e. the assumption that the QNM of the original structure forms an acceptable approximation to the perturbed configuration is apparently better justified. For both the symmetric and the asymmetric perturbation, the variational procedure of appendix 4.6, in Figure 4.5 B) and D) applied with the supermodes of the perturbed composite structure in the template, gives accurate results for the spectral transmission through the double cavity structure. 

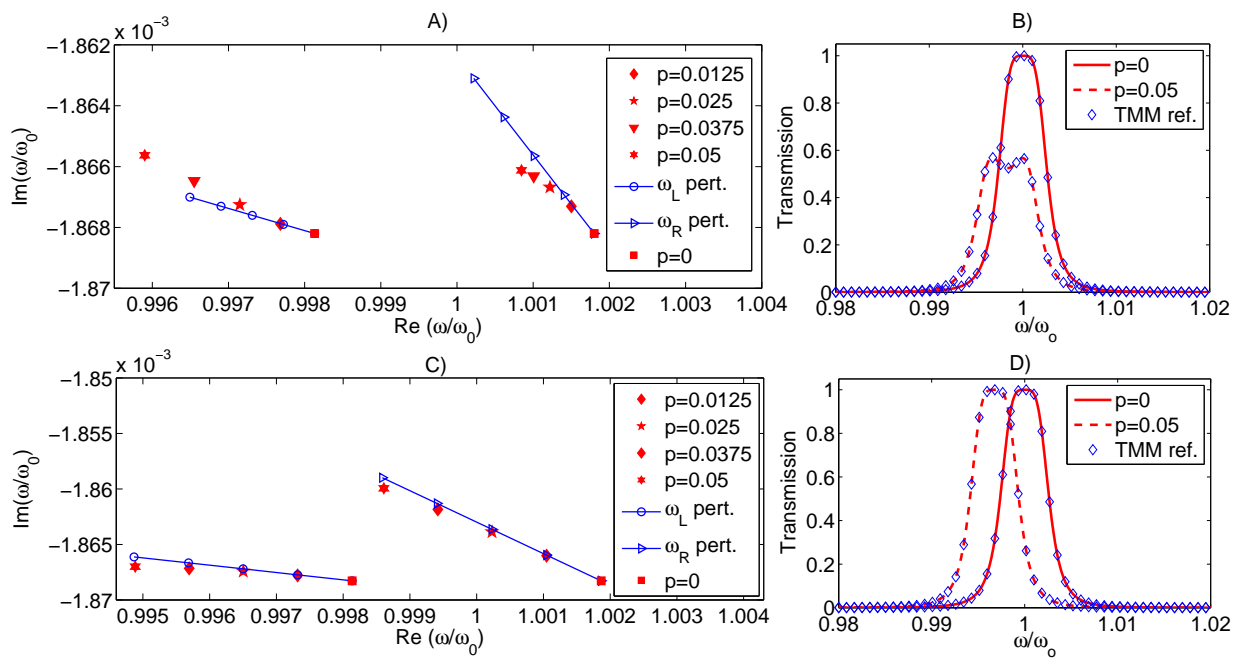

Figure 4.5: A), C): complex eigenfrequencies for the double cavity structure of Figure 4.4, direct computations and first order perturbation theory approximations; $B), D)$ : spectral transmittance, QNM approximation (appendix 4.6) based on exact QNM supermodes, and TMM reference; asymmetric (A), B)) and symmetric perturbations $(C), D)$ ).

For the asymmetrically changed double cavity configuration of Figure 4.5 A) we observed that the perturbational expression (4.15) grossly over- or underestimated the QNM eigenvalue correction. This was attributed to the fact that the underlying field template could not respond to the broken symmetry of the perturbed structure. It is thus intriguing to try a modified template that combines separate QNMs of the two individual cavities, i.e. to apply the theory of Section 4.2.1. Necessarily with this procedure one encounters a certain error already for the approximation of the QNM supermode eigenfrequencies of the unperturbed, symmetric structure (observe that this concerns a configuration with relatively low refractive index contrast and strong interaction). Still, according to Figure 4.6 A), the eigenfrequency shifts predicted by the CMT formalism cover the whole range of perturbation strengths considered here with reasonable accuracy, at least as far as real parts are concerned. Plots B) and C) of Figure 4.6 show that the eigenfunctions of the perturbed structure are indeed not even approximately symmetric.

\subsubsection{Multiple cavity structures}

First, we look at the multiple cavity structure (the PC molecule) that is formed by repeating the former single cavity structure (the $\mathrm{PC}$ atom) according to the following design rule. Repetition of the unit cell $P C A_{1}=(H L)^{M_{1}}(2 H)(L H)^{M_{1}}$, here with $M_{1}=4$, generates the molecule $\left[P C A_{1}, L\right]_{J}$, where $J$ is the number of PC atoms. The refractive indices are the same as given in Figure 4.4 for the previous 

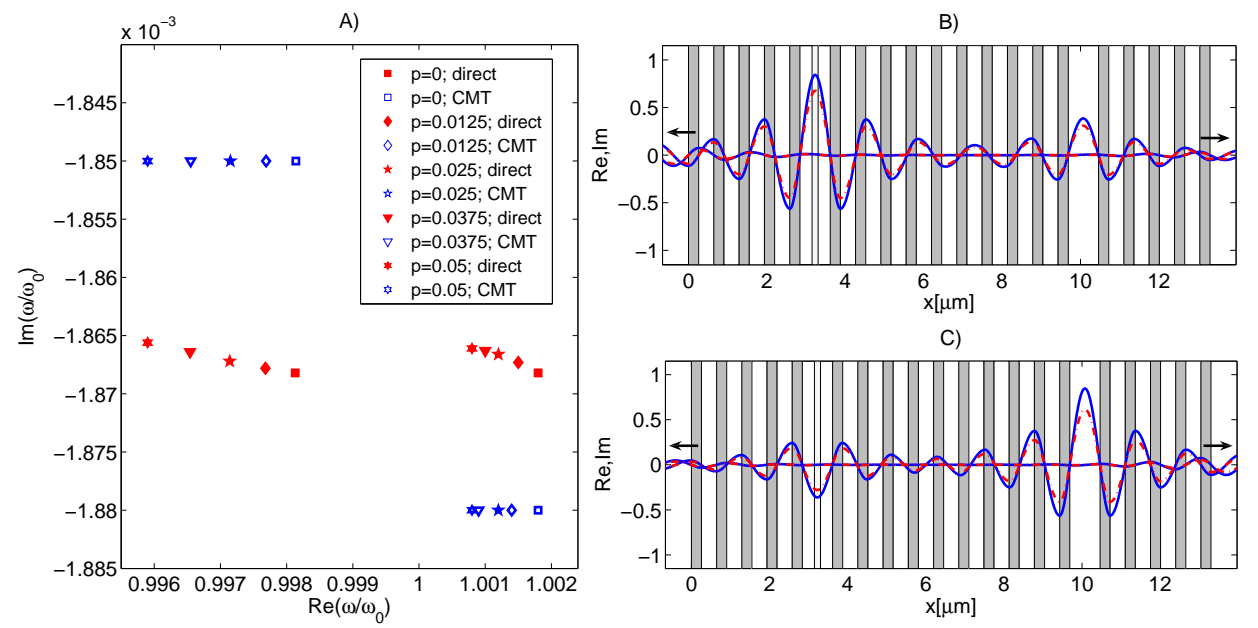

Figure 4.6: A): complex eigenfrequencies for the double cavity structure of Figure 4.4, direct TMM computations and CMT approximations, in the case of asymmetric perturbations. B), C), for or a perturbation strength $p=0.05$ : QNM profiles obtained with CMT (dashed lines) and direct computation (continuous).

example. The plots A) and B) in Figure 4.7 show the complex frequencies and the resonant transmission for PC molecules with $J=2$ and $J=3$, respectively. Obviously these $\mathrm{PC}$ molecules operate in the weak coupling regime, as is reflected in the proximity of the eigenfrequencies (A) ) and in the characteristic transmission pass-band (B) ). The transmission, estimated according to the recipes of appendix 4.6 with directly computed QNM supermodes of the molecule, is in the excellent agreement with the TMM reference. The number of relevant QNMs in the composite structure is equal to the number of PC atoms; modifications of this number permit a constructive tailoring of the transmission pass-band. For additional tuning of the transmission that might be of interest, such as ripple suppression (to optimize for a flat-top response), one could adjust the strength (number of layer pairs) of the mirrors, or add a certain degree of asymmetry to the final design $[93,6]$.

Second, we consider the molecule formed by repeating the unit cell $P C A_{2}=$ $(H L)^{M_{1}}(2 H)(L H)^{M_{2}} L(2 H)(L H)^{M_{1}}$, with $M_{1}=4$ and $M_{2}=2$ (a strongly coupled double cavity structure), coded as $\left[P C A_{2}, L\right]_{J}$. In Figure 4.7 the complex eigenfrequencies (C) ) and the spectral transmission (D)) are shown. This procedure represents the design of a multiple channeled filter with narrow bandpass transmission. By proper adjustment of the inter cavity separation (i.e. of the coupling strength), the relative position of the transmission channels can be controlled. Additional unit cells contribute to the eigenfrequency splitting in such a way that the split eigenfrequencies are close. Therefore, no additional transmission bands appear but the width of the transmission pass-bands is narrowed.

Finally, a combination of the PC atoms $P C A_{1}$ and $P C A_{2}$ leads to an even 

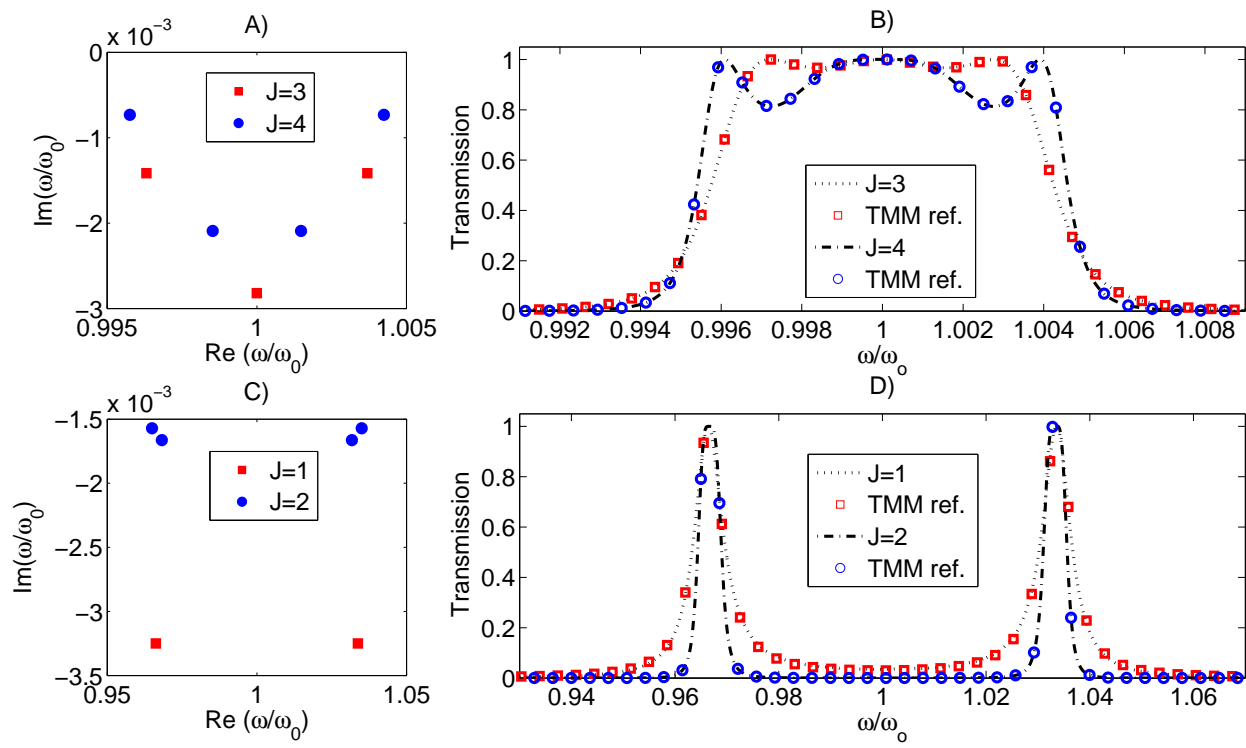

Figure 4.7: A), B): complex eigenfrequencies and transmission for weakly coupled multiple cavity structures $\left.\left.\left[P C A_{1}, L\right]_{J} ; C\right), D\right)$ : frequencies and transmission for $P C$ molecules $\left[P C A_{2}, L\right]_{J}$ formed by repeating a double cavity unit cell in the strong coupling regime.

more complex composite structure. The PC molecule is given by the sequence $P C M=\left[P C A_{1}, L, P C A_{2}, L, P C A_{1}\right]$. Figure 4.8 shows eigenfrequencies (A)) and the corresponding QNMs (B)-E)). The individual contributions of each atom to the supermode profiles of the molecule are clearly visible. The eigenfrequencies $\omega_{2}$ and $\omega_{3}$ are the product of a weak coupling between the atoms $P C A_{1}$ (the single cavity structures), according to the shape of the corresponding QNMs (D), E) ). The eigenfrequencies $\omega_{1}$ and $\omega_{4}$ originate from $P C A_{2}$ and are affected by $P C A_{1}$ only in the form of an increased confinement (i.e. a lower absolute value of the imaginary parts of the eigenfrequencies). The transmission for the composite structure exhibits a characteristic combination of both constitutive atoms. The high transmittance peaks are caused by the resonances associated with $P C A_{2}$, while the transmission resonances of $P C A_{1}$ are modulated (here they are suppressed) by the presence of $P C A_{2}$. In this case, light can not establish an efficient propagation path from the left $P C A_{1}$ to the right one, because the frequencies supported by $P C A_{1}$ are inside the attenuation region of $P C A_{2}$, see Figure 4.7.

We like to emphasize here that the QNM analysis can be very useful for an interpretation of results and for an accurate prediction of the outcome of transmission experiments, as shown in the previous paragraphs. Here, the approach establishes a sound foundation of the concept of photonic crystal molecules, that cannot be provided easily by direct TMM solutions. 

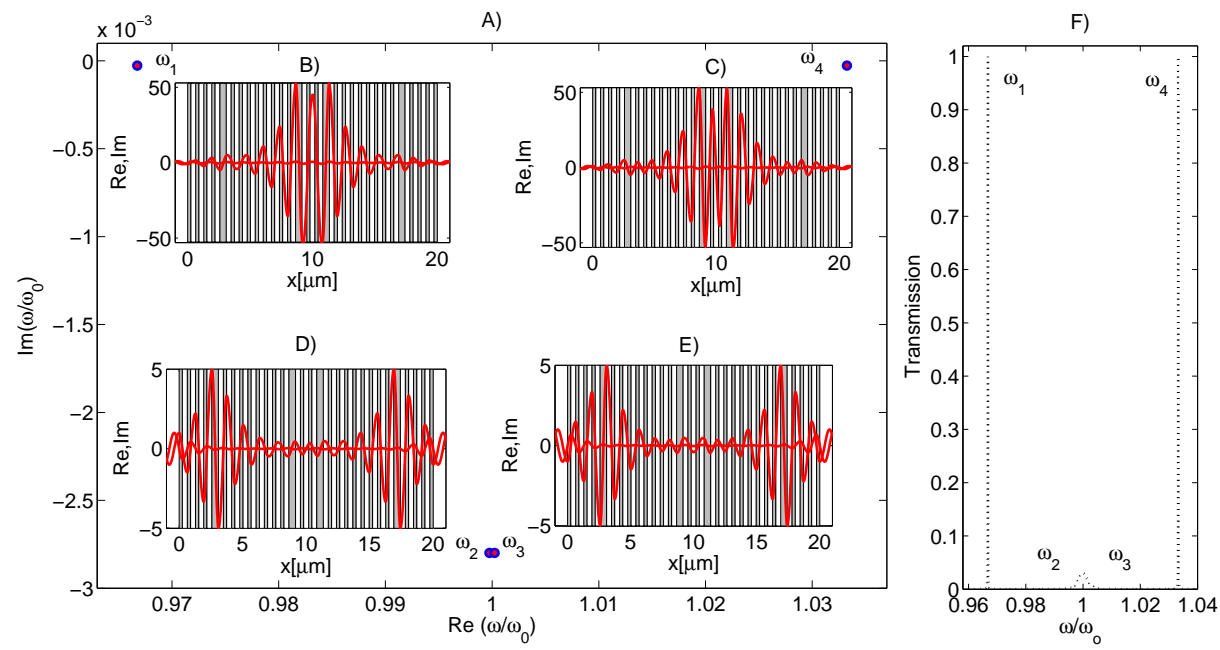

Figure 4.8: A): complex eigenfrequencies $\omega_{1}-\omega_{4}$ of the PC molecule $\left[P C A_{1}, L, P C A_{2}, L, P C A_{1}\right]$ formed by combination of the single and double cavity atoms of Figure 4.7. The insets show the corresponding QNMs, where $\omega_{1}, \omega_{2}$, $\omega_{3}$, and $\omega_{4}$ are related to profiles $\left.\left.B\right), D\right), E$ ), and $C$ ). F): spectral transmission for the composite structure.

\subsection{Conclusions}

In this paper we consider the open and finite nature of a specific class of PC structures by directly characterizing their resonance properties via an investigation of the quasi-normal mode spectrum. A variational principle for QNMs allows to approximate the eigenfrequencies and QNMs of composite multiple cavity structures by eigenfrequencies and QNMs of simpler structures. Further, a constructive, recently developed way [93] of relating a quasi-normal mode description to transmission properties of optical defect microcavities in 1D PCs is applied. Detailed remarks about alternative existing methods can be found in [93].

We specialize to defect structures that support transmission modes in the bandgap of otherwise periodical structures. Numerical examples show that the method is applicable for both symmetric and nonsymmetric layer arrangements and both weak and strong coupling between defects.

A form of coupled mode theory for finite, open 1-D PC structures is proposed, that uses directly the most relevant QNMs. Closely related, an expression for a first order perturbation correction of the complex eigenfrequencies is derived by means of variational restriction. In contrast to other methods that use different types of basis fields and rely either on a tight-binding approximation [86, 89] and/or on supercell methods $[87,88]$, with our approach the finite nature of the individual building blocks in the composite structure is fully respected.

Further, we analyzed a series of characteristic examples of multiple cavity 
structures and were able to point out characteristic features in the composite structures as originating from simpler structures. The results suggest that the notion of the photonic crystal molecules can be founded on the QNM analysis as considered here. Together with our variational approximation method, the QNM analysis offers a resourceful method for the interpretation of complex phenomena associated with the resonance properties in 1-D PC structures.

Provided that suitable QNM basis fields can be made available by analytical or numerical means, possible generalizations to 2D and 3D structures could be based on suitable functional representations of the frequency domain Maxwell equations for higher dimensions [85].

\subsection{Appendix A: Transfer matrix method}

For structures with piecewise constant refractive index distribution inside a finite spatial domain a method for solving both the transmittance and eigenvalue problems is the well known transfer matrix method (TMM) [4]. Solutions of the Helmholtz equation are given as combinations of left- and right-traveling waves in the $j$-th layer

$$
E_{j}(x)=A_{j} e^{i k_{j}\left(x-l_{j-1}\right)}+B_{j} e^{-i k_{j}\left(x-l_{j-1}\right)}
$$

for $x \in\left[l_{j-1}, l_{j}\right]$ in a region of constant index $n_{j}$ where $k_{j}=n_{j} \omega / c$ is the wave number in this layer. To connect the fields inside all layers we impose continuity conditions at the interfaces:

$$
E_{j}\left(l_{j}\right)=E_{j+1}\left(l_{j}\right) \text {, and } \partial_{x} E_{j}\left(l_{j}\right)=\partial_{x} E_{j+1}\left(l_{j}\right) .
$$

These conditions lead to a system of equations that can be represented in matrix form. Ordered multiplication of the relevant matrices connects amplitudes in each layer of the structure, as well as the amplitudes in the incidence and output regions:

$$
\left(\begin{array}{c}
A_{\text {in }} \\
B_{\text {in }}
\end{array}\right)=\left(\begin{array}{ll}
m_{11}(\omega) & m_{12}(\omega) \\
m_{21}(\omega) & m_{22}(\omega)
\end{array}\right)\left(\begin{array}{c}
A_{\text {out }} \\
B_{\text {out }}
\end{array}\right)
$$

The transmittance problem with incoming wave from the left is solved with $B_{\text {out }}=$ 0 for specified $A_{i n}$ (amplitude of the incoming wave) with given real frequency $\omega \in R$. The amplitude transmission and reflection coefficients are expressed as

$$
t(\omega)=\frac{A_{\text {out }}}{A_{\text {in }}}, \text { and } r(\omega)=\frac{B_{\text {in }}}{A_{\text {in }}} .
$$

If we choose conditions $A_{\text {in }}=B_{\text {out }}=0$, i.e. restrict the exterior solutions to purely outgoing waves, the eigenvalue problem with outgoing wave boundary conditions is addressed. With these conditions the system of equations can be nontrivially satisfied if

$$
m_{11}(\omega)=0
$$


Analytic continuation of the transfer matrix into the complex plane enables us to find solutions of (4.20) as complex eigenvalues $\omega$ [23]. By substituting the eigenvalue into the field representation (4.16) we obtain the corresponding eigenfunction, up to a complex constant. To solve (3.15) we apply a numerical iteration procedure of Newton type [61]. In cases when that method fails to converge due to closely spaced eigenvalues, we use a more powerful technique for determining complex solutions, based on the argument principle method from complex analysis [82].

\subsection{Appendix B: Variational QNM model of the transmis- sion problem}

We specialize to finite periodic structures that possess transmission properties with a bandgap, i.e. with a region of frequencies of very low transmission. Breaking the periodicity of the structure can give rise to defect resonances inside the bandgap. Approximation of the spectral transmission and of the associated field profiles for these resonances is the aim of our analysis. Therefore, we choose a field template for the transmittance problem as

$$
E(x, \omega) \simeq E_{m f}(x, \omega)+\sum_{p=1}^{N} a_{p}(\omega) Q_{p}(x),
$$

where $p$ is an index counting $N$ relevant QNMs, i.e. those with the real part of their complex frequency in the given frequency range. We showed in terms of the successful application of the template (4.21), that the transmission resonances associated with the defects are excited by the "mirror" field $E_{m f}$ of the periodic structure without defect, which for frequencies inside the bandgap is an almost completely reflected wave with only a weak tail that extends into the interior of the structure. Therefore, this template (4.21) quantifies the notion of a forced resonance response that appears because the incident wave possesses a real frequency close to the real part of the complex eigenfrequency of a suitable QNM supported by the defect structure.

This is only an approximate model for the transmittance problem in specific frequency regions, since neither $E_{m f}$ nor $Q$ satisfy all of equations (3.2)-(3.3). The residuals can be viewed as contributions from other QNMs in the complete set supported by the defect structure, that are not included in (4.21). To find the decomposition coefficients $a_{p}$, we use a variational form of the transmittance problem [25]. The transmittance problem corresponds to the equation and natural boundary conditions, arising from the condition of stationarity of the functional

$$
\begin{aligned}
\mathcal{L}(E) & =\frac{1}{2} \int_{L}^{R}\left(\left(\partial_{x} E\right)^{2}-\frac{\omega^{2}}{c^{2}} n^{2}(x) E^{2}\right) d x \\
& -\frac{i \omega}{2 c}\left(\left.n_{\text {in }} E^{2}\right|_{x=L}+\left.n_{\text {out }} E^{2}\right|_{x=R}\right)+\left.2 i \frac{\omega}{c} n_{\text {in }} A_{\text {inc }} E\right|_{x=L} .
\end{aligned}
$$


If $\mathcal{L}$ becomes stationary, i.e. if the first variation of $\mathcal{L}(E)$ vanishes for arbitrary variations of $E$, then $E$ satisfies (3.2), and (3.3),(3.4) as natural boundary conditions. Restricting the functional (4.22) to the field template (4.21), $\mathcal{L}$ becomes a function of the coefficients $a_{p}$, for given $E_{m f}$ and $Q_{p}$. The stationarity conditions then read

$$
\frac{\partial L}{\partial a_{q}}\left(a_{1}, a_{2}, \ldots, a_{N}\right)=0, \quad q=1, \ldots, N .
$$

The optimal decomposition coefficients are obtained as solutions of a linear system

$$
\mathbf{A} \mathbf{a}=-\mathbf{b}
$$

where $\mathbf{a}=\left[a_{1}, a_{2}, \ldots, a_{N}\right]^{T}$ is the vector of coefficients to be determined by solving the system of equations (4.24). $\mathbf{A}$ and $\mathbf{b}$ are calculated according to (4.21, 4.22, 4.23); explicit expressions are given in [93]. For given frequency $\omega$ one thus approximates the field profile for a transmission problem with a specific incoming wave. Spectral information (transmittance, reflectance) can be obtained by repeating this procedure for a series of frequencies. The transmittance reads

$$
T(\omega)=\frac{1}{\left|A_{\text {inc }}\right|^{2}} \frac{n_{\text {out }}}{n_{\text {in }}}\left|E_{m f}(R, \omega)+\sum_{p=1}^{N} a_{p}(\omega) Q_{p}(R)\right|^{2} .
$$

We showed in [93] that the mirror field is necessary for approximating the incoming part of the transmission field on the whole spatial region occupied by the structure. However, an additional approximation that is analytical in form can be obtained without the mirror field when only the spectral transmittance profile is considered. In cases where the underlying periodic sequence forms a good mirror, i.e. provides a high reflectance over the bandgap region, the mirror field could be omitted from the field template. This is possible because the mirror field contribution in the relevant terms of (4.24) becomes negligible for the field at the end of the structure where only outgoing waves are present. Then this approach can be seen as an alternative projection technique for a QNM expansion. 
Chapter 4. Coupled optical defect... $\quad$ 4.6. Appendix B: Variational QNM... 


\title{
Chapter 5
}

\section{Negative index metamaterials and thermal radiation}

\begin{abstract}
In this chapter we briefly review some of basic properties of the negative index metamaterials. Also, we address some novel properties of the bandgap structure and transmission spectra obtained by the introduction of NIM in the construction of the infinite and the finite multilayers. Finally we briefly review some basics concerning thermal radiation and multilayer structures.
\end{abstract}




\subsection{Negative index metamaterials}

Negative index metamaterials are artificial composites, characterized by subwavelength features and negative real part of the refractive index of the homogenized structure. Usually they are made of an ordered or random arrangement of unit cells, i.e. elementary "particles", that furnish effective electromagnetic response functions permittivity and permeability. A mathematical procedure in which the complicated microscopic electromagnetic fields existing in the inhomogeneous medium are replaced by macroscopic fields that are smoothly varying is called homogenization. Then information on the microscopic properties of the medium is discarded and the wave propagation becomes sensitive only to macroscopic, averaged properties of the effectively homogeneous media $[95,36,96]$. The validity of such a description is an issue in general, and the extraction of the effective, averaged material properties is a difficult task with non unique solution. Heuristically, the main prerequisite for the homogenization is the subwavelength nature of the elementary electric and magnetic "particles". This means that the characteristic length scales of the inhomogeneity are much smaller than the wavelength of interest [31, 97, 34].

This is in contrast to other types of artificial structures and media introduced recently in optics such as Photonic Band-Gap materials, where the features are in the order of the wavelength, thus representing mesoscopic entities. Homogenized effective electromagnetic response functions have been used for these structures, despite of loose physical foundation, as a means of approximation for numerical and qualitative investigations [7].

Negative index metamaterials were theoretically predicted in 1967 by Veselago [32]. The concept relies on the analysis of the wave propagation in media with simultaneously negative real parts of both permeability and permittivity, for which the refractive index has to be chosen with a negative real part as well. Practically, it seems that this type of material does not appear in nature, and therefore it has to be artificially made.

The main obstacle in exploiting these ideas earlier were technological limitations that did not allow the fabrication of the proposed materials and structures. However, in the year 2000 Smith and Schurig performed an experiment in the microwave range demonstrating the feasibility of the metamaterial concept with simultaneously negative permittivity and permeability in a certain frequency range and negative refraction [98]. They used a composite medium consisting of thinwires that furnish negative permittivity and split-ring resonators that furnish negative permeability.

The true explosion of the field came with seminal work of Sir John Pendry and his proposal of a device named "perfect lens" [99]. This is a device based on a slab of the negative index media that resonantly amplifies the evanescent field of an object and reconstructs both the far-field (propagating waves) and near-field (evanescent waves) components and thus enables the perfect reconstruction of an image. The perfect lens operates in a regime that exceeds the diffraction limit 
for an ordinary imaging device. This initial concept started much effort in the research of metamaterials in the past several years. Although a perfect lens has been shown to be of limited applicability in real-world situations, many theoretical and experimental results have been obtained in the direction of imaging beyond diffraction limit and improving and modifying other electromagnetic devices and processes [100, 101, 102].

The major breakthrough is yet to come from possible realizations of negative index metamaterials for the optical frequencies. The key obstacle is in the fact that for the optical range there are no efficient materials with magnetic (permeability) response in the linear regime. Negative electric (permittivity) response is achievable with metallic materials and metallo-dielectric structures [103]. The advent of nanotechnologies could lead to a breakthrough in the structuring of the materials for electromagnetic and optical applications with the properties and functionalities beyond those occurring in natural materials. Negative refractive indices have been experimentally reported recently by several research groups, see $[40,40,41,104,105]$ and references therein. However, all designs used for optical wavelengths so far suffer from the very strong dispersion and high absorption losses.

\subsubsection{Permeability, permittivity and refractive index}

Consider harmonic wave propagation in a linear, isotropic, and homogeneous material. The Maxwell equations in the frequency domain then read

$$
\begin{aligned}
\mathbf{k} \times \mathbf{E} & =\mathrm{i} \omega \mu_{0} \mu \mathbf{H}, \\
\mathbf{k} \times \mathbf{H} & =-\mathrm{i} \omega \epsilon_{0} \epsilon \mathbf{E} .
\end{aligned}
$$

While the Helmholtz equation is

$$
\nabla^{2} \mathbf{E}(\mathbf{r}, \omega)+k^{2} \mathbf{E}(\mathbf{r}, \omega)=0
$$

with the plane wave solution

$$
\mathbf{E}(\mathbf{r}, \omega)=\mathbf{E}(\omega) \mathrm{e}^{i \mathbf{k} \cdot r},
$$

where

$$
|\mathbf{k}|^{2}=k^{2}=\frac{\omega^{2}}{c^{2}} \epsilon(\omega) \mu r(\omega) .
$$

Assume for the moment that permittivity and permeability are real. It is evident from (5.3) that if $\epsilon$ and $\mu$ are of the same sign the medium supports propagating waves, while for opposite signs of the response functions the waves are evanescent with purely imaginary wave vector. This is depicted in Figure 5.1 and can be used for a classification of different materials, depending on the type of waves that medium supports. 


\begin{tabular}{|c|c|}
\hline$\varepsilon<0, \mu>0 \quad \mu$ & $\varepsilon>0, \mu>0$ \\
\hline Metals & Dielectrics \\
\hline Evanescent waves & Propagating waves \\
\hline$\varepsilon<0, \mu<0$ & $\varepsilon>0, \mu<0$ \\
\hline $\begin{array}{l}\text { Negative Index } \\
\text { Metamaterials }\end{array}$ & Magnetic materials \\
\hline Propagating waves & Evanescent waves \\
\hline
\end{tabular}

Figure 5.1: Permittivitypermeability $(\epsilon-\mu)$ diagram (real parts)for classification of materials and types of supported waves.

For plane wave solutions it becomes important to choose appropriately the sign in the expression for the wave number

$$
k=\frac{\omega}{c} n=\frac{\omega}{c} \sqrt{\epsilon \mu}
$$

where $n=\sqrt{\epsilon \mu}=n_{r e}+i n_{i m}$ is the complex refractive index. Allowing now for complex $\epsilon$ and $\mu$ in the relation

$$
\left(n_{r e}+i n_{i m}\right)^{2}=\left(\epsilon_{r e}+i \epsilon_{i m}\right)\left(\mu_{r e}+i \mu_{i m}\right)
$$

we have to consider conditions for the appropriate choice of the complex square root. For simplicity, let us consider linearly polarized waves with $z$-dependent electric and magnetic fields

$$
E(z)=E_{0} \exp \left(i \frac{\omega}{c} n z\right), H(z)=\frac{n}{\mu_{r} Z_{0}} E_{0} \exp \left(i \frac{\omega}{c} n z\right)
$$

where $Z_{0}=\sqrt{\mu_{0} / \epsilon_{0}}$ is the intrinsic impendance of vacuum. Hence, the timeaveraged Poynting vector reads

$$
S_{z}=\frac{1}{2} \operatorname{Re}\left[\mathbf{E} \times \mathbf{H}^{*}\right]=\operatorname{Re}\left[\frac{n}{\mu_{r}}\right] \frac{\left|E_{0}\right|^{2}}{2 Z_{0}} \exp \left(-2 \frac{\omega}{c} n_{i m} z\right)
$$

In case of a passive possibly attenuating material one has to require $n_{i m} \geq 0$ $[2,30]$. From , this condition leads to

$$
n_{r e}=\frac{\epsilon_{r e} \mu_{i m}+\mu_{r e} \epsilon_{i m}}{2 n_{i m}}
$$

hence $n_{r e}<0$ if an only if $\epsilon_{r e} \mu_{i m}+\mu_{r e} \epsilon_{i m}<0$. For causal and passive media both $\epsilon_{i m}>0$ and $\mu_{i m}>0$ have to satisfied $[2,30,106]$. Then we are left with the rule (1.38)

$$
\begin{array}{ll}
\operatorname{Re}(n)<0 & \text { if } \operatorname{Re}(\epsilon)<0 \text { and } \operatorname{Re}(\mu)<0 \\
\operatorname{Re}(n) \geq 0 & \text { otherwise. }
\end{array}
$$


If we express complex numbers $z=z_{r e}+i z_{i m}$ as $z=|z| \exp (i \varphi)$, where $\varphi=$ $\operatorname{arccot}\left(z_{r e} / z_{i m}\right)$, then

$$
n=\sqrt{|\epsilon||\mu|} \exp \left(\frac{i}{2}\left[\operatorname{arccot}\left(\frac{\epsilon_{r e}}{\epsilon_{i m}}\right)+\operatorname{arccot}\left(\frac{\mu_{r e}}{\mu_{i m}}\right)\right]\right) .
$$

According to the Maxwell equations (5.1) and (5.2) when NIMs are considered, the electric and magnetic field and wave vector form left-oriented set, in contrast to the situation with positive index $\mathbf{E}, \mathbf{H}, \mathbf{k}$, Figure 5.2. This is the reason for name left-handed materials used in part of the literature

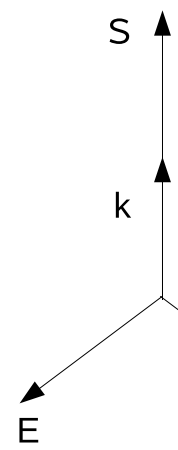

a)

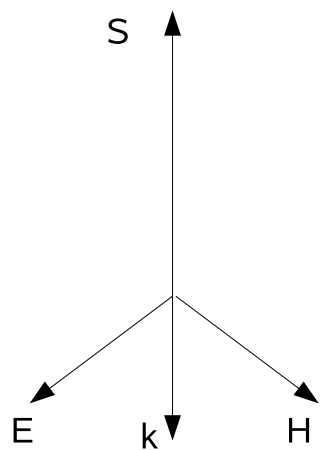

b)
Figure 5.2: $\quad$ Electric field-magnetic field-wave vector triad $[\boldsymbol{E}, \boldsymbol{H}, \boldsymbol{k}]$ and Poynting vector $\boldsymbol{S}$ for propagating electromagnetic waves, a) Positive index medium $(\epsilon>0$, $\mu>0)$ b) Negative index medium $(\epsilon<0, \mu<0)$

All causal materials are bound to be dispersive, because the electric and magnetic polarizations depend on the history of the applied fields, thus responding noninstantaneously to their influence [30]. Permeability and permittivity are in general complex functions of the frequency where the imaginary parts are related to dissipative processes in the material. Real and imaginary parts of the permeability and permittivity are connected via Kramers-Kronig relations [1,2].

Several common microscopic descriptions of materials lead to models of damped oscillators [2]. The response of the elementary oscillators to the external excitation, i.e. the time dynamic equations of motion of the polarization and magnetization, in the presence of an oscillating electric and magnetic field, can be written in the form

$$
\begin{gathered}
\partial_{t}^{2} \mathbf{P}+\Gamma_{e} \partial_{t} \mathbf{P}+\omega_{r e}^{2} \mathbf{P}=\epsilon_{0} \omega_{p e}^{2} \mathbf{E}, \\
\partial_{t}^{2} \mathbf{M}+\Gamma_{m} \partial_{t} \mathbf{M}+\omega_{r m}^{2} \mathbf{M}=\omega_{p m}^{2} \mathbf{H}
\end{gathered}
$$

where $\omega_{r e(r m)}$ is the resonance frequency of the electric(magnetic) dipole oscillators, $\Gamma_{e(m)}$ is the damping frequency, $\omega_{p e}$ and $\omega_{p m}$ may be interpreted as measures of the strength of the interaction between the corresponding oscillators and the electric and magnetic fields $[31,106]$. Hence, the frequency domain material response functions are of the form of the Lorenz oscillator

$$
\epsilon(\omega)=1-\frac{\omega_{p e}^{2}}{\omega^{2}-\omega_{r e}^{2}+i \Gamma_{e} \omega} \text { and } \mu(\omega)=1-\frac{\omega_{p m}^{2}}{\omega^{2}-\omega_{r m}^{2}+i \Gamma_{m} \omega} .
$$


Another model widely applicable for NIMs leads to a Drude-type dispersion (characteristic for metals with free electron plasma)

$$
\epsilon(\omega)=1-\frac{\omega_{p e}^{2}}{\omega\left(\omega+i \Gamma_{e}\right)}, \text { and } \mu(\omega)=1-\frac{\omega_{p m}^{2}}{\omega\left(\omega+i \Gamma_{m}\right)},
$$

where $\omega_{p e(m)}$ are the effective electric and magnetic plasma frequencies and $\Gamma_{e(m)}$ are electric (magnetic) damping factors (usually expressed as a fraction of the plasma frequency). This represents a model of an artificial electric and magnetic plasma [13].

\subsubsection{Energy density, phase and group velocity in NIM}

The expression for the wnergy density in a linear, isotropic, lossless and nondispersive medium gives a negative value for energy density if both $\epsilon$ and $\mu$ are negative. In fact (1.28) can not be used in this situation because NIMs have to be dispersive; this observation supports that requirement further.

If we consider a narrow radiation band and use a Taylor series expansion about the carrier frequency while retaining only the linear term, it is possible to derive an expression for the energy density $[2,30]$

$$
W=\frac{1}{2}\left[\frac{\partial(\omega \epsilon)}{\partial \omega} \mathbf{E}^{2}+\frac{\partial(\omega \mu)}{\partial \omega} \mathbf{H}^{2}\right] .
$$

Equation (5.17) is applicable in the transparency regions, for frequencies sufficiently far away from a resonance and with negligible absorption. Then, sufficient conditions for the energy density to be positive definite under these approximations are

$$
\frac{\partial(\omega \epsilon)}{\partial \omega}>0, \text { and } \frac{\partial(\omega \mu)}{\partial \omega}>0
$$

These are satisfied in the regions of normal dispersion far from resonances and for all dispersion relations used to model artificial electromagnetic structures such as metamaterials as well as ordinary materials [30].

Note, that (5.17) is derived under rather special conditions that may not be satisfied in general. In other situations of interest, such as a frequency regions with high absorption and anomalous dispersion, an expression for the energy density stored in the dispersive medium can not be given in a closed form [30, 31].

In fact, interactions between waves and structured media associated with NIMs are dynamical in their nature. Therefore, the steady state response of the NIMs appears only after a certain time interval necessary for all transient processes to finish. Further, dispersion has to be present to ensure positive-definiteness of the energy density and necessarily is accompanied by losses due to the Kramers-Kronig relations $[30,13]$. Therefore, according to the current understanding of NIMs, the best performance that any possible design may achieve are at best only enlarged frequency ranges of moderate or small dispersion and losses. 
The phase velocity, i.e. the velocity of propagation of a plane of constant phase reads

$$
\mathbf{v}_{p}=\frac{\omega}{k} \frac{\mathbf{k}}{|\mathbf{k}|} .
$$

It is clear that the sign of the phase velocity changes in NIMs. Therefore, sometimes term backward-wave media or backward-phase velocity media is used for materials with this property [96].

Another important quantity is the group velocity, i.e. the velocity of propagation of the envelope of a wave packet [2]

$$
\mathbf{v}_{g}=\nabla_{\mathbf{k}} \omega(\mathbf{k}) .
$$

Further, in media with negligible absorption, it can be shown that group velocity is equal to the energy flow velocity associated with the direction of the Poynting vector, which does not depend on material properties $[1,2,30]$. Thus, negative refractive index implies that the phase velocity is in the direction opposite to the direction of the group velocity. Then, it is clear that phase and energy velocity are antiparallel. Note, that this discussion is applicable only for configurations with negligible losses.

Still, all realizable NIMs are fundamentally bound to be dispersive and lossy. Hence, the simple notions regarding phase, group and energy velocity need to be modified in general. Some caution in the analysis of the wave propagation in regimes of anomalous dispersion and similar phenomena is necessary, see [30] and references therein.

\subsubsection{Photon momentum in NIM}

The mechanical momentum density vector $\mathbf{D} \times \mathbf{B}$ associated with the electromagnetic field is parallel to the local Poynting vector $\mathbf{E} \times \mathbf{H}$ both inside ordinary media and inside NIMs [30]. This suggests that the linear momentum of a photon $\mathbf{p}$ and the wave vector $\mathbf{k}$ are no longer parallel in NIMs. The linear photon momentum and the wave vector are linked via the relation

$$
\mathbf{p}= \pm \hbar \mathbf{k}
$$

where the plus sign corresponds to situations where ordinary material are considered, and the minus sign is the appropriate choice for NIMs [30]. This simple change may lead to interesting consequences such as reversal of light pressure $[32,30]$. Although, the proper definition of photon momentum for a general dispersive and lossy materials is still a bit of a problem, both in classical and quantum optics, it is applied when NIMs are considered, see [30] and references therein. We use (5.21) in chapter 6 we review briefly the derivation of the Planck's law in linear, isotropic, dispersive NIMs with negligible absorption. 


\subsubsection{Snell's law and negative refraction}

Let us consider a plane monochromatic wave

$$
\mathbf{E}_{i}=\mathbf{E}_{0 i} \mathrm{e}^{i\left(\mathbf{k}_{i} \cdot \mathbf{r}-\omega t\right)}
$$

incident on a planar interface of two media characterizied by permittivities $\epsilon_{1(2)}$ and permeabilities $\mu_{1(2)}$, see Figure 5.3. Then, the reflected wave and the transmitted wave are

$$
\begin{aligned}
\mathbf{E}_{r} & =\mathbf{E}_{0 r} \mathrm{e}^{i\left(\mathbf{k}_{r} \cdot \mathbf{r}-\omega t\right)}, \\
\mathbf{E}_{t} & =\mathbf{E}_{0 t} \mathrm{e}^{i\left(\mathbf{k}_{t} \cdot \mathbf{r}-\omega t\right)} .
\end{aligned}
$$

Magnetic field components may be obtained from (5.1) and (5.2). The wave numbers are defined via (5.5). The interface conditions accompanying Maxwell's equations have to be satisfied for the fields at all points at the interface and at all times $[1,2]$, and therefore a phase matching condition arises

$$
\left(\mathbf{k}_{r} \cdot \mathbf{r}\right)_{z=0}=\left(\mathbf{k}_{r} \cdot \mathbf{r}\right)_{z=0}=\left(\mathbf{k}_{t} \cdot \mathbf{r}\right)_{z=0} .
$$

The incident wave vector can be decomposed into its components $k_{i x}=\frac{\omega}{c} n_{i} \cos \left(\theta_{i}\right)$ and $k_{i z}=\frac{\omega}{c} n_{i} \sin \left(\theta_{i}\right)$, as well as the other wave vectors in their components, see Figure 5.3.

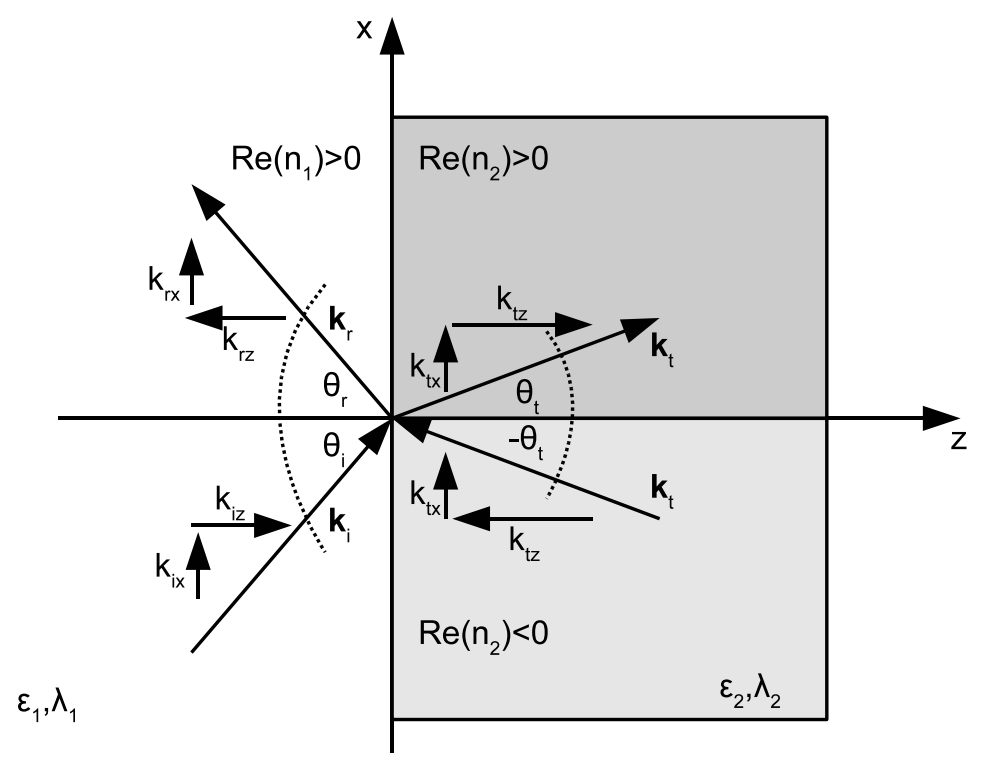

Figure 5.3: Phase-matching conditions and the positive or negative refraction at the boundary between two media.

The phase-matching (5.25) conditions on the interface between the two media leads to the laws of refraction Snell'law. The law of reflection is

$$
\theta_{i}=\theta_{r}
$$


unchanged from the situation with usual materials [30]. The Snell's Law of refraction reads

$$
n_{i} \sin \left(\theta_{i}\right)=n_{t} \sin \left(\theta_{t}\right),
$$

and it also holds for all materials, including NIMs. However, if $n_{t}<0$ then $\theta_{t}<0$ and the transmitted wave is negatively refracted. Here, negative refraction is a simple consequence of the interface conditions for the electromagnetic fields. Note, that this type of negative refraction is fundamentally different from negative refraction encountered with the Photonic Crystal structures [7]. There, the negative refraction effect arises in the diffraction process on the mesoscopic scale, because structural properties vary on the scale of the wavelength and the propagation direction of the refracted wave on the interface between homogeneous media and Photonic Crystal structure depends on the dispersion relation in a complicated manner $[9,7]$.

Expressions for amplitude reflection and transmission coefficients, called Fresnell's formulas for both TE and TM polarization, may be derived from the interface conditions. They read for TE polarization

$$
\begin{aligned}
& r_{T E}=\frac{E_{r 0}}{E_{i 0}}=\frac{\mu_{2} k_{i z}-\mu_{1} k_{t z}}{\mu_{2} k_{i z}+\mu_{1} k_{t z}}, \\
& t_{T E}=\frac{E_{t 0}}{E_{i 0}}=\frac{2 \mu_{2} k_{i z}}{\mu_{2} k_{i z}+\mu_{1} k_{t z}} .
\end{aligned}
$$

and for TM polarization

$$
\begin{aligned}
& r_{T M}=\frac{H_{r 0}}{H_{i 0}}=\frac{\epsilon_{2} k_{i z}-\epsilon_{1} k_{t z}}{\epsilon_{2} k_{i z}+\epsilon_{1} k_{t z}}, \\
& t_{T M}=\frac{H_{t 0}}{H_{i 0}}=\frac{2 \epsilon_{2} k_{i z}}{\epsilon_{1} k_{i z}+\epsilon_{1} k_{t z}} .
\end{aligned}
$$

These relations are valid for general media that may be lossy as well. It follows that the reflection coefficient may become zero for either polarization for a certain incidence angle (Brewster angle) and properly chosen parameters [30, 37, 13].

\subsection{Multilayers containing negative index metamaterials}

\subsubsection{Phase compensation effect}

The important feature of multilayers and other structures with NIMs is the phase compensation or the process of the partial or the full removal of the wave phase shift after propagating through consecutive PIM and NIM layers. It is a consequence of the sign reversal in the phase factors $\delta_{j}=k_{j z} d_{j}$ in (1.55) for the fields in the layers with NIM. For a general multilayer containing NIM, the phase compensation influences greatly specific interference pattern and modifies the spectral transmission and reflection properties [43, 107]. In fact, the majority of unusual properties associated with NIM-based structures arise from this effect [31]. 
For simplicity let us consider TE-polarization and plane wave incident on binary grating consisting of two materials $\mathrm{A}$ and $\mathrm{B}$ with material properties $\epsilon_{A(B)}, \mu_{A(B)}$ and thicknesses $d_{A(B)}$. The transfer matrix (1.55) connects amplitudes in the adjacent layers. Hence, the phase-shifts are $\delta_{A(B)}= \pm \frac{\omega}{c}\left|n_{A(B)}\right| d_{A(B)} \cos \theta_{A(B)}$ where the plus sign corresponds to the usual positive index material and minus sign to the NIMs, and $s_{A(B)}=k_{j A(B)} / \mu_{A(B)}=\sqrt{\mu_{A(B)} / \epsilon_{A(B)}} \cos \theta_{A(B)}$. The corresponding formal angles $\theta_{A(B)}$ are connected by Snell's law (5.26) to the incident angle.

Let us consider a special case of a normally incident wave on a multilayer in which slabs with $\epsilon_{A}=\mu_{A}$ are in the air $\epsilon_{B}=\mu_{B}=1$. Thus, we have $s_{A(B)}=1$ and the transfer matrix becomes purely a phase-shift matrix

$$
\left(\begin{array}{c}
A_{j} \\
B_{j}
\end{array}\right)=\left(\begin{array}{cc}
\mathrm{e}^{-i k_{j z} d_{j}} & 0 \\
0 & \mathrm{e}^{+i k_{j z} d_{j}}
\end{array}\right)\left(\begin{array}{c}
A_{j+1} \\
B_{j+1}
\end{array}\right)
$$

Therefore, the propagating wave does not experience any reflection at the interfaces between the different layers. This situation is known as perfect matching, i.e. when intrinsic impedance of the material slab is the same as the intrinsic impedance of air (free space). For a finite multilayer with $N$ layers as in Figure 1.2, the overall transfer matrix reads

$$
\left(\begin{array}{c}
A_{0} \\
r A_{0}
\end{array}\right)=\left(\begin{array}{cc}
\mathrm{e}^{-i \sum_{j=1}^{N} \phi_{j}} & 0 \\
0 & \mathrm{e}^{+i \sum_{j=1}^{N} \phi_{j}}
\end{array}\right)\left(\begin{array}{c}
t A_{0} \\
0
\end{array}\right) .
$$

where the phase shifts are $\phi_{j}=k_{j z} d_{j}=\omega / c n_{j} d_{j}$. Then, the amplitude transmission coefficient is

$$
t=\mathrm{e}^{i \sum_{j=1}^{N} \phi_{j}} .
$$

The transfer function (5.34) is an all pass filter function [6]. For a NIM-containing multilayer the overall phase shift could become zero $\sum_{j=1}^{N} \phi_{j}=0$ for $N$ even, which corresponds to full phase compensation.

Although the considered situation is idealized and can not be achieved fully due to intrinsic dispersion and losses in NIMs it represents the best example of phase compensation. In fact, a fully phase-compensated and perfectly matched multilayer represents a stack of Pendry's perfect lenses [99]. This structure would perfectly reconstruct both propagating (far field) and evanescent (near field) components from object to image, see [31] and references therein.

In other, non-ideal cases when reflections exist at interfaces between layers due to impedance mismatch, the presence of NIMs in the layer stack influences the overall phase shift.

\subsubsection{Periodic structures with NIMs and non-Bragg bandgaps}

Consider an infinite periodic structure with alternating PIMs and NIMs layers. The mathematical model outlined in chapter 1 is fully applicable for the bangap analysis of these structures. Hence, the dispersion relation (1.72) determining bandgaps 
and pass bands can be obtained by solving (1.71). Phase compensation and possibilities for adjusting the reflection properties of interfaces up to the ideal perfect matching case, can influence the bandgap structure greatly. The enlarged number of parameters with respect to just one for ordinary all-dielectric and metaldielectric structures enables extended photonic bandgap engineering. Several new phenomena exist that cannot be observed for structures with ordinary media.

It can be easily shown for a binary stack as in previous subsection that (1.71) reads

$$
\cos \left(K_{B} \Lambda\right)=\cos \left(\left|k_{A}\right| d_{A}\right) \cos \left(\left|k_{B}\right| d_{B}\right) \pm \frac{Z_{A}^{2}+Z_{B}^{2}}{2 Z_{A} Z_{B}} \sin \left(\left|k_{A}\right| d_{A}\right) \sin \left(\left|k_{B}\right| d_{B}\right) .
$$

where $Z_{A(B)}=\sqrt{\mu_{A(B)} / \epsilon_{A(B)}}$ is the characteristic impedance of layers $\mathrm{A}$ and $\mathrm{B}$. The minus sign corresponding to all-PIMs structure and the plus sign to PIMsNIMs structure [42].

Some specific conditions may be drawn directly from (5.35). If the optical thicknesses are equal $\left|n_{A}\right| d_{A}=\left|n_{B}\right| d_{B}$ then the right side of the (5.35) is always greater then 1 . Thus, the spectral bandgap exists at all frequencies except for points $\left|k_{A}\right| d_{A}=\left|k_{B}\right| d_{B}=m \pi$, where $m=0,1,2 \ldots$ is an integer. If in addition structure is perfectly matched, $Z_{A}=Z_{B}$, then (5.35) reduces to $\cos \left(K_{B} \Lambda\right)=1$ and for all frequencies propagating waves are supported, i.e. bandgaps are not present. Additionally a structure may be designed to be omnidirectional, i.e. exhibit bandgap for both polarizations [108].

An especially interesting situation arises with the so-called zero-n bandgap, existing only for stack of alternating PIMs and NIMs layers [46]. It is a new type of photonic band gap, different from the Bragg gap, that occurs when the averaged effective refractive index of the whole structure equals zero

$$
\langle n\rangle=\frac{n_{A} d_{A}+n_{B} d_{B}}{\Lambda}=0 .
$$

If dispersion is considered then (5.36) defines a characteristic frequency where this bandgap appears. The zero-n gap is less sensitive to a change of the lengthscale (i.e. scaling the period) than the usual Bragg bandgaps, and also exhibit reduced sensitivity to $t$ small scale randomness in the material properties and the thicknesses [46, 45]. It appears in periodic [46], quasi-periodic [47] and aperiodic structures [48]. Structures operating in the zero-n regime may be effective mirrors with nearly omnidirectional and unit reflectance while thicknesses may be well below the operating wavelength [45]. In addition, with properly adjusted parameters these structures may possess complete omnidirectional bandgaps [44]. However, some results suggest, that the condition $\langle n\rangle=0$ may be only necessary but not sufficient condition for bandgap opening; this holds especially when truncated, finite multilayers are considered [109].

Defects in an otherwise periodic structure may give rise to defect states in the bandgaps. These defect states may appear in the ordinary Bragg bandgaps or spe- 
cial zero-n bandgaps, where they appear to inherit some properties such as lower sensitivity to the incidence angle $[45,110]$.

\subsubsection{Transmission spectra of finite multilayers with NIM}

Any finite structure can be characterized by it's spectral reflection and transmission. As we already pointed out phase compensation can lead quite special spectral characteristics of PIM-NIM multilayers. Most basic properties outlined for infinite periodic structures are still present in the finite case.

Consider spectral transmission under normal incidence for a series of finite multilayers with material properties $\epsilon_{A}= \pm 2.25, \mu_{A}= \pm 1, n_{A}= \pm 1.5$ and $\epsilon_{B}=\mu_{B}=n_{B}=1$, while the external medium is assumed to be vacuum, the same as material B. Plus and minus signs correspond to the choice of PIMs or NIMs media. The thicknesses of the layers are chosen to be quarter-wavelength $\left|n_{A}\right| d_{A}=\left|n_{B}\right| d_{B}=\lambda_{0} / 4$ with respect to a target wavelength $\lambda_{0}=(2 \pi c) / \omega_{0}$. Figure 5.4 depicts transmission coefficients and field profiles computed using the transfer matrix method described in section 1.2.2.

First, in Figure 5.4 A) the spectral transmittance for periodic multilayers, i.e. Bragg mirrors, coded as $(A B)^{8} A$, is depicted. Note, the difference between $P-$ $N$ and $P-P$ multilayers arising from the phase compensation in the $P-N$ structure. Here, the bandgap extends over all frequencies with the exception of the discrete points (for the present finite structure regions around even multiples of $\left.\omega / \omega_{0}\right)$ as explained in the previous subsection. Characteristic interference pattern in the transmittance of the ordinary $P-P$ Bragg mirror is removed. The unit transmittance for a fully compensated and perfectly impedance matched multilayer (here the stack with $\epsilon_{A}=\mu_{A}$ ) is also depicted. In Figure 5.4 B) the distribution of the field profile modulus $|E(z)|$ in $P-N$ multilayer for frequency $\omega / \omega_{0}=2$ is shown. It has the characteristic of an extended transmission resonance field distribution, similar to the ordinary $P-P$ structure. It shows that the origin of the transmission resonance is the same as in the multilayer with ordinary media. Note that at the frequencies of the transmission resonance the layer thicknesses satisfy the condition $\left|n_{A}\right| d_{A}=\left|n_{B}\right| d_{B}=m \lambda_{0} / 2$ with $m=0,1,2 \ldots$, i.e. the condition for absentee layers in the periodic multilayer [4].

Second, in Figure 5.4 C) the spectral transmittance for symmetric defect $P-N$ multilayer, coded as $(A B)^{4} 2 A(B A)^{4}$ is shown. Introduction of the defect in the otherwise periodic structure gives rise to a transmission resonance in the middle of the bandgap. This defect resonance is of Fabry-Perot type, i.e. the periodic parts on both sides of the multilayer play the role of mirrors enclosing the effective cavity represented by the defect. This interpretation is further supported by inspecting the distribution of the field profile modulus shown in Figure 5.4 D), where the characteristic field localization and the enhancement in the vicinity of the defect can be observed. 

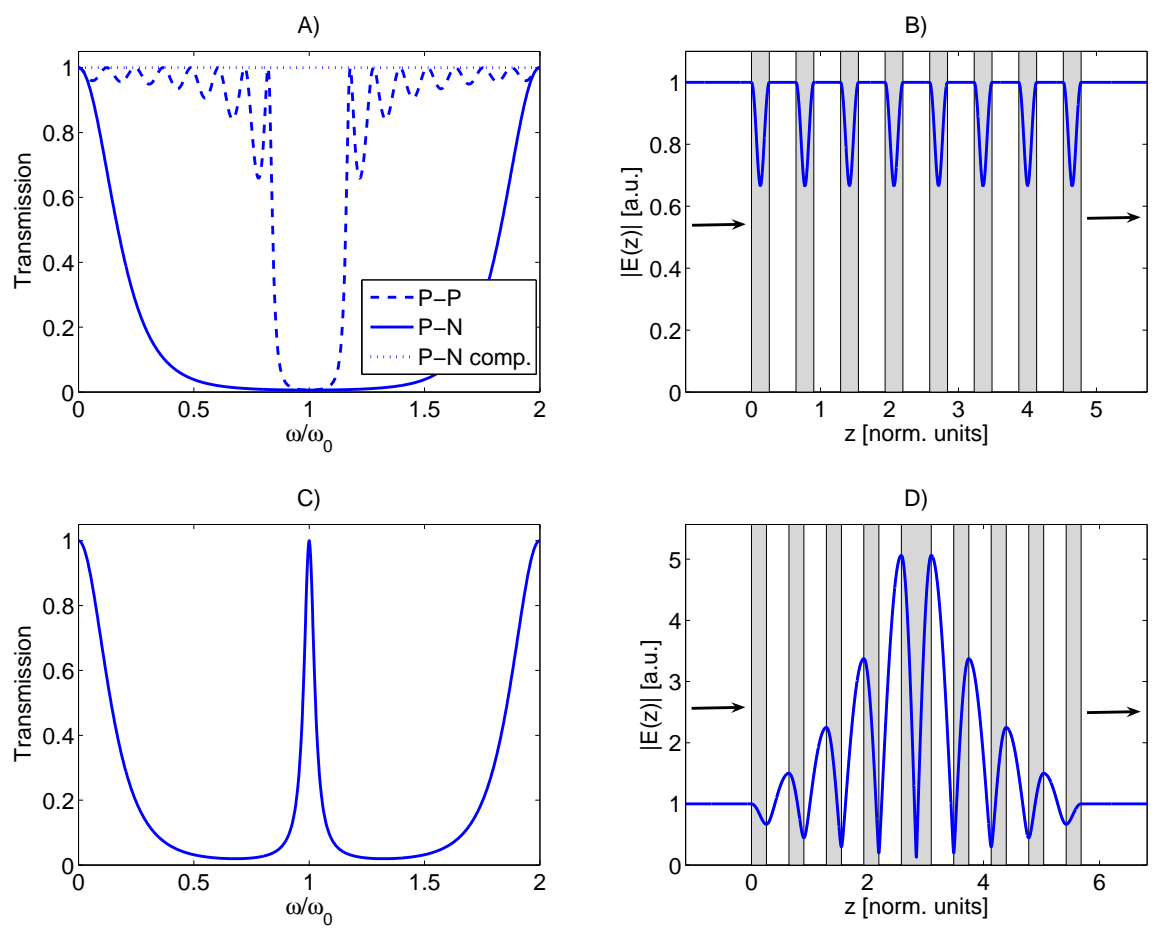

Figure 5.4: A) Spectral transmission for the PIM-PIM, PIM-NIM and fully compensated and perfectly matched $\left(\epsilon_{A}=\mu_{A}\right)$, PIM-NIM multilayer, B) Local field profile modulus for PIM-NIM multilayer at transmission resonance $\omega=2 \omega_{0} C$ ) Defect PIM-NIM structure spectral transmission, D) Local field profile modulus for defect resonance $\omega=\omega_{0}$.

\subsection{Thermal radiation and NIM materials}

Thermal radiation is electromagnetic radiation associated with thermal phenomena and heat transfer in material objects. It represents the fundamental physical process of radiative energy transfer associated with microscopic events of electromagnetic radiation emission induced by electron transitions in atoms, through phonon transitions associated with molecular rotational and vibration modes and crystal lattice oscillations [49, 50, 111]. In terms of wavelengths, it covers the whole electromagnetic spectrum, including the ultraviolet, visible and infrared range.

The investigation of physical phenomena associated with thermal radiation in nature an engineering applications is an important part of both theoretical and applied science.

From the application point of view regarding NIM, one of the interesting questions is the spectral and angular distribution of thermal radiation in systems with multilayer structures containing NIMs, for purposes of thermal radiation control 
and tailoring through the design of such multilayers.

The material properties and physical phenomena associated with thermal radiation and heat transfer can be described only by taking into account both the classical and quantum nature of electromagnetic waves and their interaction with the material objects $[49,50,3,51,111]$. However, in the case of NIM-containing multilayers the situation is well modelled by classical electrodynamics, i.e. the propagation of electromagnetic waves through optical structures. The relevant quantum physical processes are taken implicitly, while our model deals with the interaction of electromagnetic waves with matter using phenomenological and macroscopic effective parameters.

\subsubsection{Blackbody radiation and Planck's law}

An important concept when dealing with the thermal radiation is the blackbody or the perfect absorber (emitter). This denotes an object that absorbs or emits all incident electromagnetic radiation at all frequencies, with any polarization and from all directions. The blackbody as a perfect absorber is a standard with which real absorbers are compared. The blackbody is also a perfect emitter at every frequency and in all directions, while the total radiated power is a function of temperature only. These properties follow from the thermodynamic principle of the detailed energy balance in thermal equilibrium $[49,50]$.

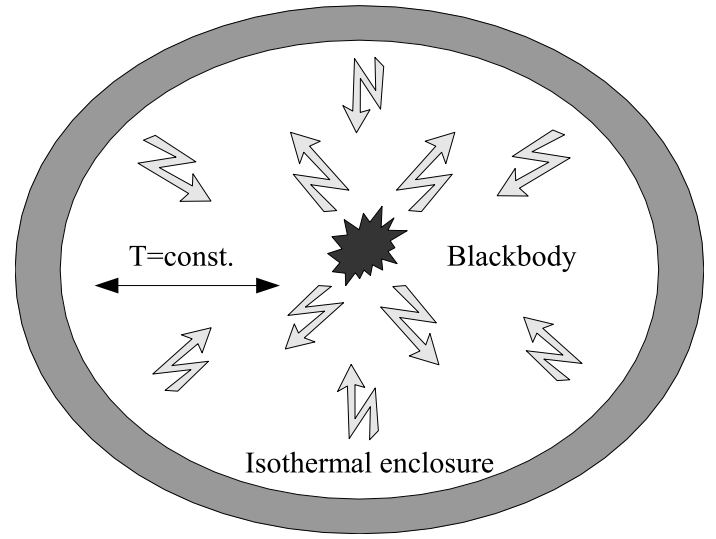

Figure 5.5: Blackbody: material object in thermal equilibrium with the isothermal enclosure at a constant temperature $T$.

The blackbody is an idealized object and can be only approximated by real bodies. As illustrated in Figure 5.5 the usual approach to such an approximation in experimental situations is to consider an absorber in an enclosure (cavity) with perfectly absorbing boundaries. For such an object in thermal equilibrium the absorption and emission rates will be equal for the radiating walls of the enclosure and for the absorber itself. Thus an isothermal situation is achieved. In this case a constant temperature may be assigned both to the enclosing surface and to the object inside the cavity. If a small pinhole is made in the cavity wall, the electromagnetic radiation emerging from it will be very close to the radiation in the cavity, i.e. it will be the nearly perfect blackbody radiation $[49,50]$. 
The blackbody power spectral distribution for EM radiation in thermal equilibrium at a macroscopic temperature $T$ and with frequency $\omega$ within homogeneous, isotropic space is described by the Planck law [51, 50]

$$
\rho^{B B}(\omega, \beta)=\frac{\omega^{2}}{2 c^{2}} \frac{\hbar \omega}{\mathrm{e}^{\hbar \omega \beta}-1}
$$

where $\beta=1 /\left(k_{B} T\right)$ and $k_{B}$ is the Boltzmann constant and $\hbar$ the reduced Planck constant.

\subsubsection{Kirchhoff's law for thermal radiation}

A phenomenological description of the macroscopic interaction between thermal radiation and matter includes the processes of reflection, transmission and absorption. If radiation is regarded as an electromagnetic wave, it may be only reflected from the surface, transmitted through the material object or absorbed within it, see Figure 5.6. As already seen in Chapter 1, energy conservation may be quantified through three phenomenological dimensionless quantities: the reflectance $R$, the transmittance $T$ and the absorptance $A$; all these quantities are defined as ratios of the corresponding spectral power densities, usually described by the respective Poynting vectors $[49,50,51]$. Energy conservation then requires that $R+T+A=1$.

Further, all material objects emit radiation within their volume and the radiation may escape from the surface into the surrounding space. To quantify the emissive properties, we define an additional phenomenological quantity $E$ called emittance. It represents the ratio between the energy emitted from the surface to the energy emitted by a blackbody at the same temperature. The blackbody emittance equals unity.

The relation between the emissive and the absorptive properties of a material object in thermal equilibrium is expressed by the Kirchhoff law. It states that the spectral emittance and the spectral absorptance of a material object in thermal equilibrium with its surroundings are equal for a given temperature and for every frequency, direction and polarization: $E(\omega, T)=A(\omega, T)$. This law can be obtained from the thermodynamical principle of detailed energy balance in the equilibrium that imposes an equality between the amount of energy being absorbed and emitted per frequency at a given temperature $[49,50,51]$. It is strictly correct only for thermal equilibrium in an isothermal enclosure. However, experimental observations show that it approximates well the majority of practical situations where a local thermodynamic equilibrium is maintained.

The thermal equilibrium of the whole system depicted in Figure 5.6 dictates energy conservation and through the Kirchhoff law imposes a relation between the following macroscopic quantities:

$$
E(\omega)=A(\omega)=1-R(\omega)-T(\omega)
$$




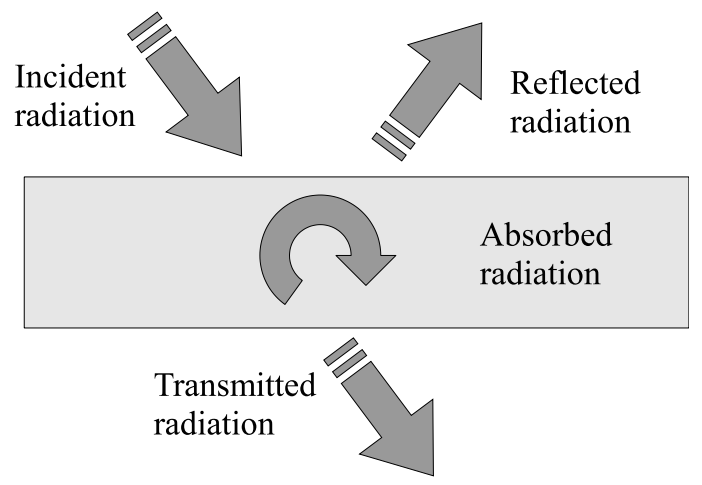

Figure 5.6: Absorption, reflection and transmission of radiation incident on a material.

Then, the spectral power distribution can be obtained by multiplying the blackbody power spectrum from Planck's law (5.37) by the calculated emittance:

$$
\rho(\omega, \beta)=E(\omega) \rho^{B B}(\omega, \beta) .
$$

Equation (5.38) is fundamental for the analysis of the emissive properties of material objects. It establishes the basis of the indirect method for computing the thermal emission properties of arbitrary material objects through determining their respective absorption. In [112] the emission properties of highly dispersive and absorptive photonic crystals are calculated directly and then compared with the predictions of the indirect method. The results suggests that indeed, in thermal equilibrium, the indirect method gives an excellent agreement with the simulations based on the direct method.

Thermal radiation seen as electromagnetic waves may have two different polarizations through the emittance $E_{T E}$ and $E_{T M}$ for TE and TM polarizations respectively. Unpolarized thermal radiation (blackbody radiation) is assumed to have equal portions of both polarizations $E=1 / 2\left(E_{T E}+E_{T M}\right)$. We consider both polarized and unpolarized emittance.

\subsubsection{Thermal radiation antennas with multilayer structures}

A thermal radiation antenna is a device that emits (absorbs) thermal radiation in a certain frequency range and into (from) a certain direction. Thus, such a structure possesses the properties of both selectivity and directionality, similar to the basic traits of electromagnetic radiation antennas.

The theoretical foundations for photonic bandgap (PBG)-based thermal radiation control have been outlined in [52]. Periodic structures with an intrinsic photonic bandgap alter the thermal radiation spectrum by modifying the photonic density of modes. Thermal radiation is suppressed at frequencies inside the photonic bandgap, and enhanced at the frequencies of transmission resonances. In this way a spectral redistribution of thermal power is achieved. This enables a control over thermal emission processes. In the case of 1D structures such control is readily implemented by the available thin-film technologies. 
A simple and straightforward approach to the computation of the modified spectral emittance is to apply the mentioned indirect method [52]. It may be applied to determine the dependence of absorption both on the frequency and on the incident radiation angle. The indirect method has been widely applied in literature to investigate the possibilities of thermal radiation control by $1 \mathrm{D}, 2 \mathrm{D}$ and $3 \mathrm{D}$ PBG structures, see $[113,114,115,55,56]$ and references therein.

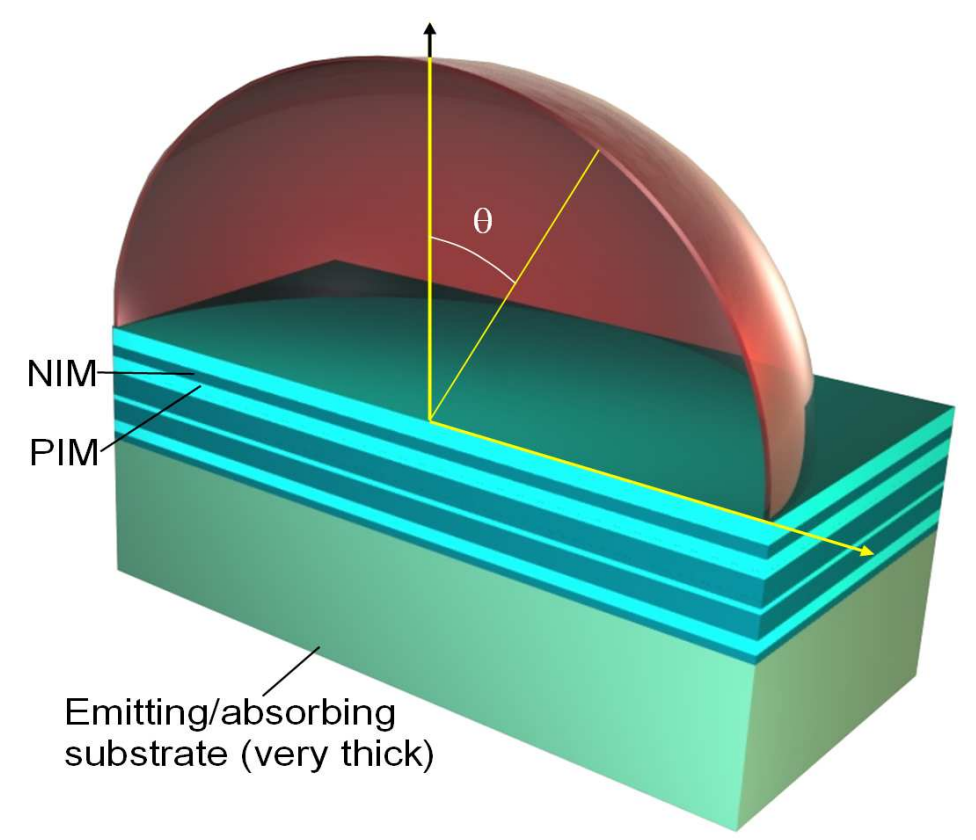

Figure 5.7: A general passive multilayer filtering system for thermal radiation control. The multilayer positioned on the top of a thick absorbing substrate enables spectral and angular distribution shaping. The actual ordering of materials shown in the multilayer will depend on the concrete design.

The design of thermal sources with the emittance enhanced in a narrow solid angle through the application of a multilayer filter has been of interest in the recent period $[53,54]$. The indirect method enables the design of a thermal radiation control system by tailoring the properties of the multilayers applied as spectral and angular filters. All structures used so far were either all-dielectric or metaldielectric multilayer coatings that enhanced or suppressed thermal emission of absorptive/emissive substrates [54]. These designs proved the practical feasibility of spectral and angular control of radiation from thermal sources in the IR range. Thin film technology has been a sound foundation for this task. The possibilities offered by periodic all-dielectric or metal-dielectric PBG media [13] may be expanded by the introduction of negative index metamaterials (NIM) [116, 117, 118]. The advances in the technology of nanostructured materials heralded NIMs for the optical 
range, see [13] and references therein. This offered new degrees of freedom in the so far theoretical design of structures for thermal radiation control.

Indeed, the investigation of multilayers consisting of alternating dielectric (positive index material, PIM) and NIM layers showed novel properties convenient for emittance/absorptance tailoring [116, 117, 118]. For instance, widening and flattening of the spectral emittance was demonstrated, while at the same time the angular dependence was much weaker [116]. Further possibilities for emittance/absorptance tailoring are introduced by utilizing NIMs-containing pre-fractal [117] and quasi-periodic multilayers [119, 118]. Excellent selectivity and directionality properties were observed in these structures, when considered as thermal radiation antennas $[117,118,120]$.

Figure 2 shows a general passive NIM-containing multilayer filter for thermal radiation control. It consists of a combination of positive and negative refractive index strata. This multilayer may be periodic, quasi-periodic or aperiodic and may contain one or more defect layers. This configuration may be seen as a thermal radiation antenna.

In chapters 7 and 8 we investigate the NIM-containing multilayers deposited on a thick absorbing substrate. These structures simultaneously perform spectral filtering and angular redistribution of thermal radiation. 


\title{
Chapter 6
}

\section{Transmission spectra of Thue-Morse multilayers containing negative index metamaterials}

\begin{abstract}
$^{1}$
We study the optical transmission spectra in one-dimensional aperiodic ThueMorse multilayers composed from alternating layers of media with positive and negative refractive index. We examine the influence of phase compensation on the spectral transmission for both on-axis and off-axis wave propagation. The origin of the transmission resonances and their relation with the field localization are analyzed. Nondispersive and lossless, as well as realistic dispersive and weakly lossy materials are considered.
\end{abstract}

\footnotetext{
${ }^{1}$ This chapter is adapted from: M. Maksimovic, Z. Jaksic, Transmission spectra of Thue-Morse multilayers containing negative index metamaterials, Acta Physica Polonica A,Vol. 112, No. 5, pp. 1055-1060, 2007
} 


\subsection{Introduction}

Negative refractive index metamaterials (NIM) are artificially structured media with sub-wavelength features and with simultaneously negative dielectric permittivity and magnetic permeability $[31,38,13]$. The direction of the Poynting vector $\mathbf{S}$ in NIM is opposite to that of the wavevector $\mathbf{k}$, i.e. electric, magnetic field and wave vectors $[\mathbf{E}, \mathbf{H}, \mathbf{k}]$ form a left-oriented triplet. This is the reason why such media were dubbed "left-handed materials", contrary to the conventional "righthanded materials" (positive refractive index, PIM). Large efforts have been dedicated to the NIM research in the recent years $([31,38,13]$ and references therein). The operating wavelengths have been progressively decreasing and negative refractive behavior has been observed in the optical range [105].

Multilayers with NIM part and with periodic [42, 46], quasi-periodic (Fibonnaccitype) [121], or pre-fractal (Cantor-type) [122, 117] layer arrangements, attracted attention due to their many peculiar properties with both theoretical and practical interest.

We investigate non-periodic one-dimensional stacks composed of alternating layers of media with positive and negative refractive index. The stacks follow a design rule based on the model aperiodic Thue-Morse (T-M) substitution sequence [16, 17, 123, 124, 125]. All-PIM T-M type multilayers possess interesting and useful spectral properties, such as self-similarity, spectral scalability and welllocalized multiple resonances in their optical transmission spectra $[16,17,123$, 124, 125]. We employ the Transfer Matrix Method as outlined in chapter 1, section (1.2.2) for numerical computations and analysis [15]. Some results on T-M multilayers with NIM are obtained in [47, 48] with the prediction of a new type of a non-Bragg, zero-n bandgap (where the averaged value of the refractive index in the structure is zero). We dedicate our attention to the resonances in the transmission spectra and the field distributions associated with them for finite structures with both on-axis and of-axis wave propagation. The angular dependence of the transmission spectra and of the resonances robustness with respect to the phase shift modulation are investigated. Transmission spectra of T-M multilayer with dispersive and lossy NIM parts are considered.

\subsection{Theory and results}

A Thue-Morse substitution sequence can be defined via a binary alphabet $\{A, B\}$ and a set of simple mapping rules $A \rightarrow A B, B \rightarrow B A$, with the symbol $A$ as an initiator. The first several T-M generations are $A, A B, A B B A, A B B A B A A B$, $A B B A B A A B B A A B A B B A$, etc.

A schematic presentation of T-M multilayers and of a single PIM-NIM interface is shown in Figure 6.1. Even-order T-M multilayers possess a mirror symmetry in refractive index distribution. The number of elements in the sequence increases in the $n$-th generation as $2^{n}$, while the number of different elements $A$ 
and $B$ remains equal. We take two refractive indices $\left(n_{A}, n_{B}\right)$ and physical lengths $\left(d_{A}, d_{B}\right)$ that correspond to $A$ and $B$ in the T-M sequence. The refractive index in the incident and in the output medium is assumed to be equal $n_{0}=1$.

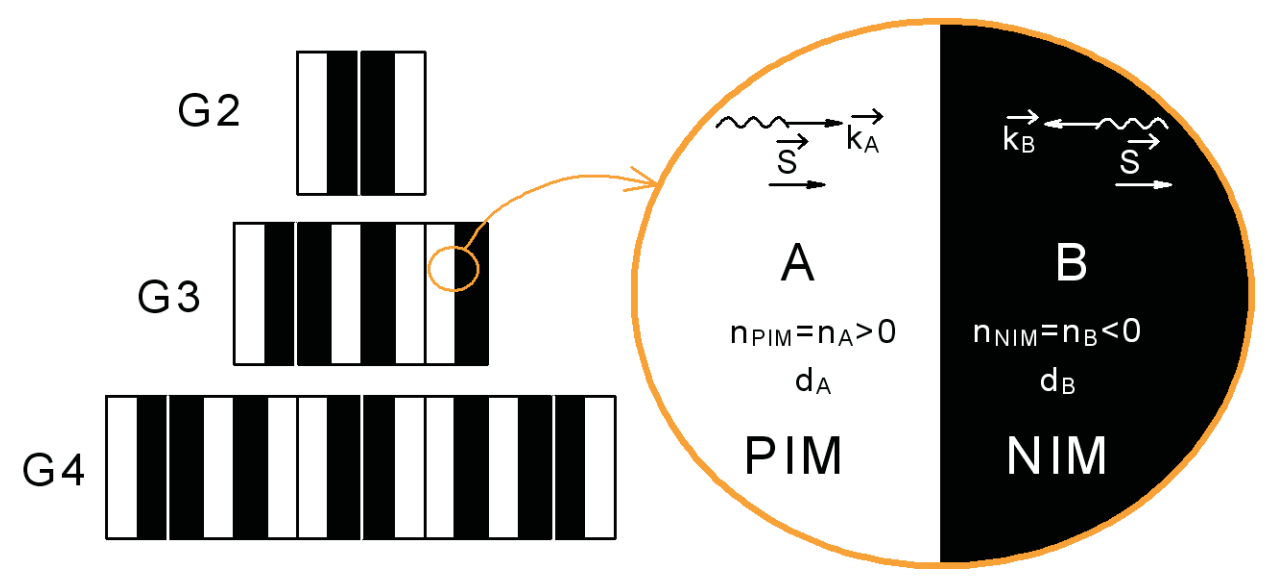

Figure 6.1: Left: aperiodic structures composed of NIM and PIM strata arranged as Thue- Morse sets for second, third and fourth generation; right: schematic presentation of a negative index-positive index material interface.

First, we chose frequency independent values of refractive indices $n_{A}=1.41$ $\left(\epsilon_{A}=2, \mu_{A}=1\right), n_{B}=-2\left(\epsilon_{B}=-4, \mu_{B}=-1\right)$, for the purpose of comparison with the prior results in literature without a loss of generality. We introduce the reduced optical thickness $n_{A} d_{A}=\alpha \lambda_{0}, n_{B} d_{B}=\beta \lambda_{0}$, with the phase shifts in the corresponding layers given as $\delta_{A}=2 \pi \alpha \Omega \cos \left(\Theta_{A}\right)$ and $\delta_{b}=2 \pi \beta \Omega \cos \left(\Theta_{B}\right)$ , where $\Omega=\lambda_{0} / \lambda_{0}=\omega / \omega_{0}$ is the normalized frequency/wavelength with $\omega_{0}$ and $\lambda_{0}$ being reference frequency and wavelength.

In the case of oblique incidence the angles of propagation $\Theta_{A}, \Theta_{B}$ are obtained via Snell's law $n_{0} \sin \left(\Theta_{0}\right)=n_{A} \sin \left(\Theta_{A}\right)=n_{B} \sin \left(\Theta_{B}\right)$, where $\Theta_{0}$ is the incidence angle in the input medium. When $\alpha=\beta=0.25$ we are dealing with a quarter- -wavelength optical thickness, which is the usual choice in literature.

The interplay between the order and the phase compensation (partial or full removal of the phase shift after propagating through a PIM-NIM structure) greatly affects the spectral resonances in the stacks based on different ordering schemes. Periodic multilayers with NIM exhibit wide band gaps and flattened transmission [42] [43], as shown in Figure 6.2 a). On the other hand T-M multilayers exhibit a similar behavior with the distinctive feature of the existence of resonances in the middle of the Bragg band gap, as seen in Figure $6.2 \mathrm{~b}$ ). Multiple resonances in the spectra of all-PIM periodic or T-M multilayers are not present in the PIM-NIM structures due to phase compensation, see Figure $6.2 \mathrm{c}$ ) and d). While the quasiperiodic and pre-fractal Cantor NIM-containing multilayers possess self-similar and scalable spectra for higher generations [122],[117] in T-M multilayers these spectral properties are absent, even in the dispersionless and lossless case. 

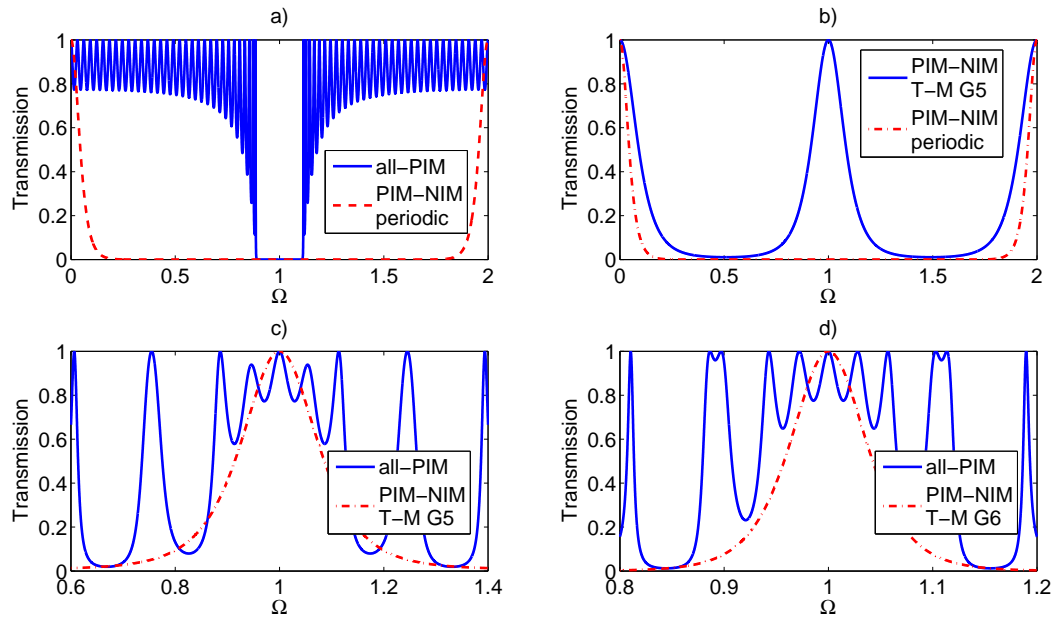

Figure 6.2: Direct comparison of transmission spectra for all-PIM (solid) and PIMNIM (dashed) periodic multilayer with 32 layers in stack a) and 5th generation T-M multilayers $b$ ) and equal quarter-wavelength optical thickness. Transmission spectra for 5th generation c) and 6th generation d) T-M multilayer.

a)
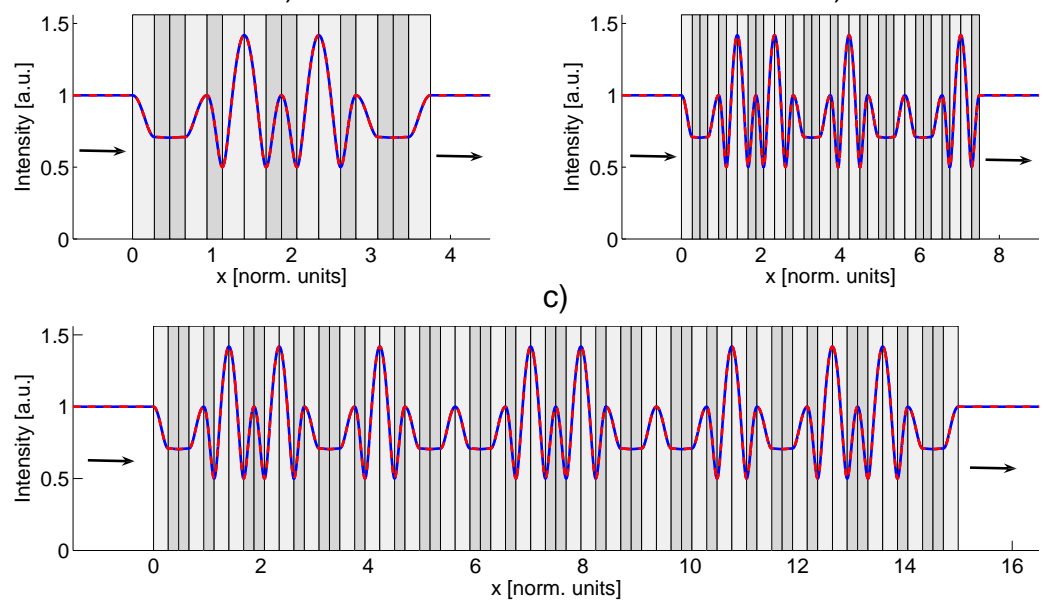

b)

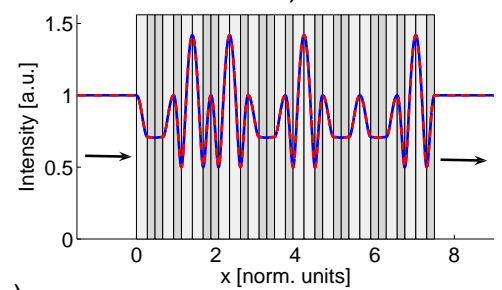

c)

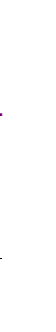

Figure 6.3: Intensity profile for normal incidence in all-PIM and PIM-NIM T-M multilayer for 4th a), 5th b) and 6th generation c) and $\Omega=1$.

In PIM-NIM T-M multilayers only a midgap resonance at $\Omega=1$ is present for normal incidence, due to a special spatial correlation in the T-M multilayer. The field modulus distribution is identical for both all-PIM and PIM-NIM structures and follows the structure of the T-M sequence, as shown in Figure 6.3. Alternating symmetric and asymmetric arrangements of layers in successive generations of 
the T-M multilayers determines the nature of the spectral resonances, i.e. perfect transmission exists in mirror symmetric structures even for oblique incidence. In asymmetric multilayers a perfect transmission resonance at $\Omega=1$ exists only for normal incidence and the quarter-wavelength optical thickness (equal phase shifts).

The resonance shift and the pronounced angular dependence, leading to the appearance of multiple resonances for oblique incidence, as shown in Figure 6.4 A) and B) is in contrast with some predictions that the transmission spectra of PIM-NIM structures are generally less sensitive to the incidence angle [42, 46, 43]. The number and the positions of transmission resonances are very sensitive to the modulation of the optical thickness $[124,125]$ which is clearly seen in Figure 6.4 c) for a 5th generation T-M multilayer (higher generations show similar behavior).

All NIM realized until now are dispersive and lossy, thus finally we choose more realistic and very strong Drude-type dispersion relations

$$
\epsilon(\omega)=1-\frac{\omega_{p e}^{2}}{\omega\left(\omega+j \Gamma_{e}\right)}, \quad \mu(\omega)=1-\frac{\omega_{p m}^{2}}{\omega\left(\omega+j \Gamma_{m}\right)}
$$

where $\omega_{p e(m)}$ are the electric (magnetic) artificial plasma frequencies and $\Gamma_{p e(m)}$ are electric (magnetic) damping constants (which can be expressed as a fraction of the plasma frequency [31]. We chose $\omega_{p e}=\omega_{p m}=\omega_{p}$ and $\Gamma_{p e}=\Gamma_{p m}=\Gamma_{p}=$ $\omega_{p} 10^{-6}$, thus the refractive index of the NIM part reads

$$
n(\omega)=\sqrt{\epsilon(\omega) \mu(\omega)} \approx 1-\frac{\omega_{p}^{2}}{\omega^{2}}+j 10^{-6} \text { and } n(\Omega) \approx 1-\frac{\Omega_{p}^{2}}{\Omega^{2}}+j 10^{-6}
$$

We assumed very small but still present losses, thus avoiding an electromagnetic nihility (material parameters locally equal to zero). Parameters for the NIM part are chosen in a such way that the physical thickness is given according to the condition $\left|n_{B}\right| d_{B}=0.25 \lambda_{0}$ for $n_{B}(\Omega=1)=-2$ (and $\Omega_{p}^{2}=3$ ), while the parameters in the PIM part are the same as before. In this way both modulations of the scattering strength (refractive index contrast) and the phase shift are present [124, 125]. Transmission spectra with many resonances appear Figure 6.4 d), but without obvious special spectral properties arising from the order or the material properties.

\subsection{Concluding remarks}

We have presented transmission spectra for the Thue-Morse multilayers com- posed from alternating layers with positive and negative refractive indices. In contrast to other non-periodic NIM-containing multilayers where self-similar and scalable spectra occurred for higher generations, for T-M multilayers these spectral properties do not appear, even in the dispersionless and lossless case. While the periodic structures with NIM exhibit wide band gaps, T-M multilayers exhibit transmission resonances located at the midgap frequency of the periodic multilayer. The field distribution at the resonance frequency suggests a common origin in both all-PIM 
a)
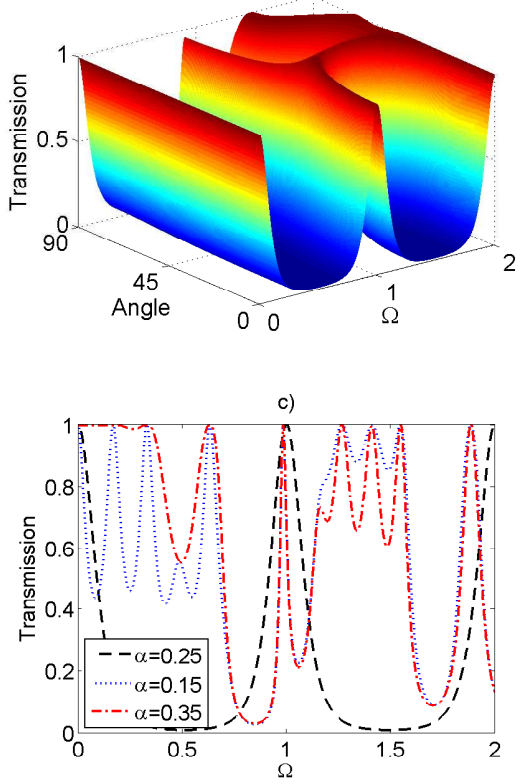

b)
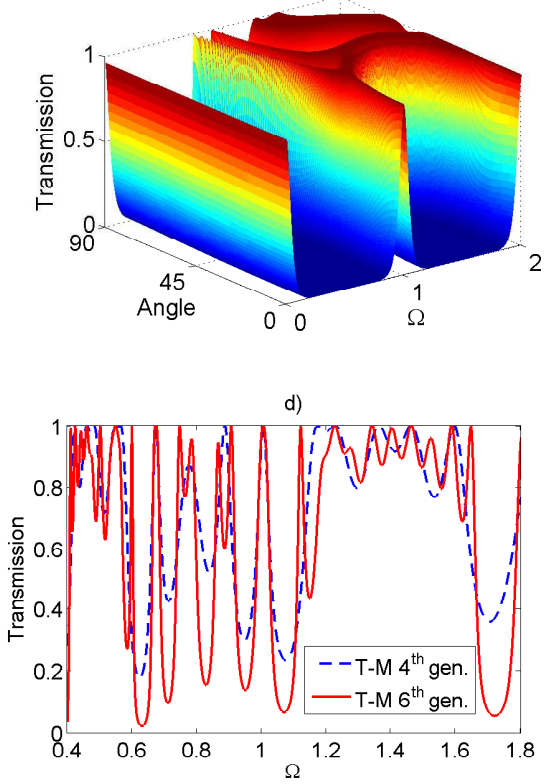

Figure 6.4: Transmission spectra for oblique incidence: the 5th generation a) and the 6th generation T-M multilayer $b$ ). Transmission spectra for varying layer thickness parameter $\alpha$ (with $\alpha+\beta=0.5$ ) at normal incidence and 5th T-M multilayer c). Transmission spectra for normal incidence and with (strong) Drude-type frequency dispersion in the NIM part and small loss d)

and NIM-containing multilayers connected with the existence of special spatial correlation irrespective of internal mirror symmetry. The position of this resonance is very sensitive to the incidence angle and the phase-shift modulation, suggesting that the phase compensation is most effective in equal-phase shift structures. The introduction of realistic material dispersion in the NIM material introduces both phase shift and scattering strength modulation in the structure. This increases the number of transmission resonances even under normal incidence conditions and effectively diminishes the influence of the aperiodic order and the phase compensation. The prospect of a weak material dispersion in the NIM leads to a potential for the application of the optical phenomena associated with aperiodic order. 


\title{
Chapter 7
}

\section{Thermal radiation and 1D periodic structures containing negative index metamaterials}

\begin{abstract}
${ }^{1}$
We investigate modification of the thermal radiation power spectrum in $1 D$ periodic structures containing negative index materials. We utilized an approach based on the Kirchoffs second law and applied the transfer matrix method to calculate emittance and to obtain the power spectrum of the periodic structure on top of a thick absorbing substrate. We analyzed both on-axis and off-axis radiation.
\end{abstract}

\footnotetext{
${ }^{1}$ This chapter is adapted from: M. Maksimovic, Z. Jaksic, Modification of thermal radiation by periodical structures containing negative refractive index metamaterials, Physics Letters A, Vol. 342, No. 5-6, pp. 497-503, 2005
} 


\subsection{Introduction}

Negative index metamaterials (NIM) are artificial composite subwavelength structures with effective electromagnetic response functions (permittivity and permeability) artificially tuned to achieve negative values of their real part [31]. These materials were theoretically predicted by Veselago [32], experimentally confirmed by Smith [126] and both experimentally and theoretically investigated by many different teams in past years.

The underlying principle in constructing NIM relies on the appearance of effective permittivity and permeability both lower than zero in the same well defined frequency band. The analyticity of refractive index regarded as a complex function and the causality principle require that the real part of the refractive index also be negative [106]. A consequence is that the product of the electric and magnetic field vectors is antiparallel with the wave vector, i.e. we deal with backward waves whose phase velocity is antiparallel with Poynting vector, while electric, magnetic field and wave vector form a left-oriented set. This is the reason why such structures are sometimes called "left-handed materials".

Many interesting phenomena not appearing in natural media were predicted and observed in double negative materials. These include negative refraction (the reversal of the Snell's law), perfect lensing [99], the appearance of subwavelength resonant cavities [33], reversal of Cherenkov radiation [30] and many other application in applied electromagnetics [97],[37].

An interesting topic of investigation is the distribution of electromagnetic modes in layered structures incorporating negative index materials. The use of conventional photonic crystal structures to modify thermal radiation was investigated by Cornelius and Dowling [52]. Subsequent theoretical and experimental results on the same topic include $[113,114,115,55,56,53,54,127]$. In the case of wave propagation through a structure consisting of a both positive refractive index material (PIM) and NIM layers a very important phenomenon of phase compensation occurs, which may be described as a partial or complete removal of phase shift of an electromagnetic wave propagating through a PIM-NIM structure [12].

In $[30,128]$ a modification of Planck law in NIM was derived relying on a simple quantized field description. There are few other papers dealing with the quantum field description of NIM-related phenomena and some interesting phenomena that arise from it, like the modification of spontaneous emission and super-radiance effect [129, 130, 131, 132].

In this chapter we investigate the modification of thermal radiation power spectrum by one-dimensional structures incorporating both NIM and conventional materials, emphasizing influence of phase compensation. We analyze periodic 1D structures and include both normal and oblique wave incidence. We use an indirect method based on the second Kirchhoff's law for thermal radiation to investigate the emittance of blackbody when a multilayer structure incorporating NIM is used as a filter on a thick blackbody. In our calculations we use the well known transfer matrix technique $[1,2,4]$ 


\subsection{The Planck law in negative index metamaterials}

Since metamaterials are structured on a subwavelength scale, it is assumed that their magnetic and electric response can be described by effective permeability and permittivity . A practice often met in literature when investigating NIM is to analyze the cases with frequency independent $\epsilon$ and $\mu$ as a first approximation of the real materials [43],[46],[45, 45]. However, realizable metamaterial must be dispersive and lossy in order to preserve causality principle [30, 31].

For the sake of simplicity we assume that both effective permittivity and permeability are of the same form

$$
\epsilon(\omega)=1-\frac{\omega_{p e}^{2}}{\omega\left(\omega+i \Gamma_{e}\right)}, \text { and } \mu(\omega)=1-\frac{\omega_{p m}^{2}}{\omega\left(\omega+i \Gamma_{m}\right)},
$$

and the effective electric and magnetic plasma frequencies $\omega_{p e(m)}$ and electric (magnetic) damping factors $\Gamma_{e(m)}$ are equal $\omega_{p e}=\omega_{p m}=\omega_{p}$ and $\Gamma_{p e}=\Gamma_{p m}=$ $\Gamma_{p}$. This choice simplifies the form of $\epsilon$ and $\mu$. The refractive index of NIM $n=\sqrt{\epsilon \mu}$ is thus

$$
n(\omega)=1-\frac{\omega_{p}^{2}}{\omega(\omega+i \Gamma)} .
$$

In our calculations, we assume that damping is negligible, which can be acceptable approximation in certain frequency range. When lossy metamaterial is considered, a common assumption in literature is that the damping factor is given as a fraction of plasma frequency [31].

An expression for the Planck radiation law in NRM media was obtained in [128] by following a simple quantized-field description for radiation in negativeindex material, which was assumed to be isotropic, dispersive and absorptionless at frequencies of interest. The approach was based on modified Einstein coefficients of spontaneous emission and absorption in the light of a simple electric dipole transition picture [51, 11] Similar results were obtained in [129, 130, 131, 132].

The same result can be obtained by a simple textbook approach $[51,111]$, which is valid for any medium described by dispersive refractive index and thus does not make a distinction between NIM and ordinary dispersive media. We apply it in the following manner. The density of photon states per volume of a photon set occupying a range of impulses $(\mathbf{p}, \mathbf{p}+d \mathbf{p})$ is $d G(p)=2\left(4 \pi p^{2} d p / h^{3}\right)$, where $\mathbf{p}= \pm \hbar \mathbf{k}$ and $\mathbf{k}$ is the photon wave vector; the multiplier 2 is due to the number of polarizations and $h^{3}$ stems from Heisenberg relations. We assume standard boundary conditions at the boundaries of the volume [51]. The mean number of photons is governed by Bose-Einstein distribution [111],thus the density of photons is

$$
d N(p)=\frac{2}{h^{3}} \frac{4 \pi p^{2} d p}{\exp \left(c p / k_{B} T\right)-1} .
$$

where $k_{B}$ is Boltzmann constant and $T$ is temperature. Further, $p=\hbar \omega n(\omega) / c$ and $d p=\hbar \omega \gamma(\omega) n(\omega) / c$, where $\gamma(\omega)=n(\omega)+\frac{d(\omega n(\omega))}{d \omega}$. These relations 
together with (7.3) lead to expression for density of photons per unit frequency $d N(\omega) /(d \omega)$. Then, the spectral energy density defined is

$$
\rho(\omega)=\hbar \omega \frac{d N(\omega)}{d \omega}=\frac{\hbar \omega^{3}}{\pi^{2} c^{3}} \frac{n^{2}(\omega) \gamma(\omega)}{\exp \left(\hbar \omega / k_{B} T\right)-1} .
$$

Expression (7.4) is the Planck's law in homogeneous, isotropic, dispersive NIM media with negligible absorption. It can be seen that (7.4) differs form the Planck law for vacuum only in factor depending on dispersive properties of the medium:

$$
\rho^{N I M}(\omega) / \rho^{\text {vacuum }}=n^{2}(\omega) \gamma(\omega) .
$$

For the dispersion described by (7.2) modification factor for spectral power density of equilibrium radiation for the space filled by NIM reads

$$
n^{2}(\omega) \gamma(\omega)=\left(1-\left(\frac{\omega_{p}}{\omega}\right)^{2}\right)^{2}\left(1+\left(\frac{\omega_{p}}{\omega}\right)^{2}\right)
$$

\subsection{Thermal radiation and multilayers containing nega- tive index metamaterials}

We consider a system in thermal equilibrium at a given temperature, its radiation having a Planck's blackbody (BB) spectrum. From the point of view of prospective practical applications, the blackbody radiation may be modified using a photonic crystal filter and thus altering the spectral emissivity of the BB radiator and/or changing the angular distribution of the radiation. This was done in [52] for the case of purely positive-media structures.

In a most general case the photonic crystal filter may have a full $2 \mathrm{D}$ or $3 \mathrm{D}$ periodicity (or even be quasi-periodic) [7]. According to conditions outlined in [52], qualitative predictions in that case can be from 1D model.

To calculate the modification of thermal radiation, it is necessary to determine the thermal emittance $\mathrm{E}$ of the photonic crystal. This is done by an "indirect" method based on the Kirchhoff's law of detailed balance. According to it a material's emittance in thermal equilibrium is proportional to its absorbtance, and for a blackbody they are equal, see chapter 5 and references therein. The absorptance is defined by the reflection and transmission coefficient $E=A=1-R-T$.

Once the emittance is obtained, its multiplication by the Planck spectral power density gives the power spectrum of the PBG emitter $\rho^{P B G}(\omega)$ in terms of its emittance $E(\omega)$ and the blackbody spectrum $\rho^{B B}(\omega)$ given by 7.4

$$
\rho^{P B G}(\omega)=E(\omega) \rho^{B B}(\omega)
$$

Fig. 1 represents a photonic band gap (PBG) structure selected to modify the mode density of radiation of the emitting substrate $\mathrm{S}$. In a general case it contains both positive index materials and NRM. The structure inhibits thermal emittance 


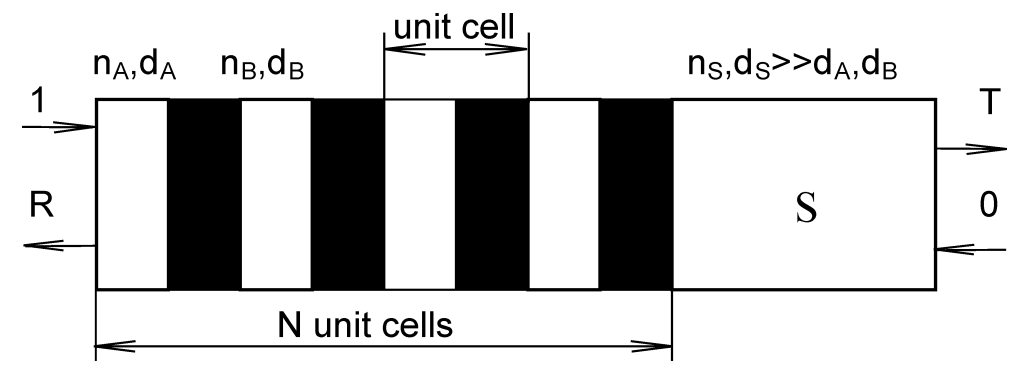

Figure 7.1: Basic 1D multilayer structure for emissivity control.

from the substrate at frequencies within the PBG, but enhances it at the band-edge. This result is confirmed for the case of all-dielectric and metal-dielectric positive index PBG materials both theoretically [52] and experimentally [54, 127].

The structure is composed from two media with refractive indices $\left(n_{A}, n_{B}\right)$ and a geometrical thickness $\left(d_{A}, d_{B}\right)$, A and $\mathrm{B}$ denoting the conventional and the NIM slabs, respectively. The structure is deposited on a thick substrate $(d>>\lambda)$ with an index $n_{S}$. We chose layers with a quarter-wavelength optical thickness $n_{A} d_{A}=n_{B} d_{B}=\lambda_{0} / 4$. Hence, the phase shifts in the corresponding layers are $\delta_{A}=(\pi / 2) \Omega$ and $\delta_{B}=(\pi / 2) \Omega$ where $\Omega=\lambda_{0} / \lambda=\omega / \omega_{0}$ is normalized frequency. The entire structure is surrounded by a medium $n_{0}$ (air or vacuum).

We chose a quarter-wavelength optical thickness for our layers to establish a connection with prior experimental and theoretical work and to directly compare our results to those previously published. More specifically, one encounters the same choice of individual layers thickness in literature on absorptance and emittance tailoring by PBG structures [52] but also in papers on NRM-caused phase compensation and its application for antireflection coatings, high-reflective coatings, transmission filters, and many other functionalities [43],[46, 45]. While there is no fundamental reason not to use a different optical thickness, quarter wavelength appears to be the most frequently used approach for different optical applications and one gets a clear physical picture without a loss of generality. Only a similar spectral behavior could be obtained by different choice of optical thickness [4], [43], but it remains a question of practical implementation for a concrete design.

The transfer matrix technique which includes material dispersion and absorptive losses $[1,4]$ can be used to compute transmission and reflection coefficients and the power spectrum. We apply it using interface matrices $\mathbf{M}_{A B}$ ( at the interface between media $\mathrm{A}$ and $\mathrm{B}$ ) and propagation matrices $\mathbf{M}_{p}$ (where $p \in\{A, B, S\}$ ).

$$
\begin{aligned}
& \mathbf{M}_{A B}=1 / 2\left[1+n_{B} / n_{A} 1-n_{B} / n_{A} ; 1-n_{B} / n_{A} 1+n_{B} / n_{A}\right] \\
& \mathbf{M}_{p}=\left[\exp \left(-i \delta_{p}\right) \quad 0 ; 0 \exp \left(+i \delta_{p}\right)\right], \quad \delta_{p}=2 \pi n_{p} L_{p} / \lambda .
\end{aligned}
$$

In the case of oblique incidence the above matrices retain the same form, but one has to substitute $n_{A(B)} \rightarrow n_{A} \cos \left(\theta_{A(B)}\right)$ for corresponding refractive in- 
dices in the propagation matrices for both TE- and TM-polarization, and $n_{A(B)} \rightarrow$ $n_{A(B)} \cos \left(\theta_{A(B)}\right)$ for TE-polarization and $n_{A(B)} \rightarrow n_{A(B)} / \cos \left(\theta_{A(B)}\right)$ for TMpolarization in the interface matrices. The overall transfer matrix for the chosen structure is given as a product of the interface and the propagation matrices $[1,4]$. The transmission and the reflection coefficient of the given structure follow from the overall transfer matrix which has the form $\mathbf{M}=\left[\begin{array}{llll}m_{11} & m_{12} ; & m_{21} & m_{22}\end{array}\right]$ and are expressed through the formulas

$$
T=\frac{1}{\left|m_{11}\right|^{2}} \text { and } R=\left|\frac{m_{21}}{m_{11}}\right|^{2} .
$$

In our investigation we consider isotropic media for both non-dispersive and dispersive cases.

One should note here that no plasmon modes interacting with the incident plane wave and changing emittance spectra were taken into account in this paper. The reason is that a propagating plane wave incident on a flat surface cannot excite surface plasmons regardless of its incidence angle, since the plasmon modes must have a larger momentum at the same frequency for all energies considered [31]. Thus no influence of the plasmon mode to the emittance characteristics can be expected. To change this situation, one would have to provide an additional momentum to disturb the plasmon mode, e.g. to use surface roughness, a grating structure or similar. This is valid both in the case of positive and negative index materials [31].

\subsection{Results and discussion}

Figure 7.2 shows the calculated emissivity of 1D PBG structures versus frequency and angle of incidence for different polarizations. Figure 7.2 a) shows the emissivity of an all-dielectric PBG material for unpolarized case. We obtained similar dependencies for the all-dielectric structure for s- and p-polarizations (not shown here). Figure 7.2 b)-d) show the calculated emissivity of NIM-containing 1D PBG structures for different polarizations.

The dependence in Figure 7.2 a) illustrates a problem pertinent to all positivematerial PBG filters: such structures can either have an optimum performance in a very narrow wavelength range for all incident angles, or for a larger wavelength range, but for a very limited spatial angle.

This is not the case with the NIM-containing filters. Figure $7.2 \mathrm{~b}$ )-d) shows that the angular dependence in emittance spectrum is much less prominent than in ordinary PBG structures. This points out to the possibility of designing efficient NIM filters almost insensitive on the radiation propagation angle. Structures containing NIM influence differently the thermal radiation spectrum in comparison to ordinary media PBG structure. The suppressed region of thermal radiation is wider, and the spectral characteristics more flat, i.e. without sharp oscillation typical for positive index materials. The influence of the angle of incidence is less noticeable than for the corresponding ordinary structures. The emittance shows no ripples and 

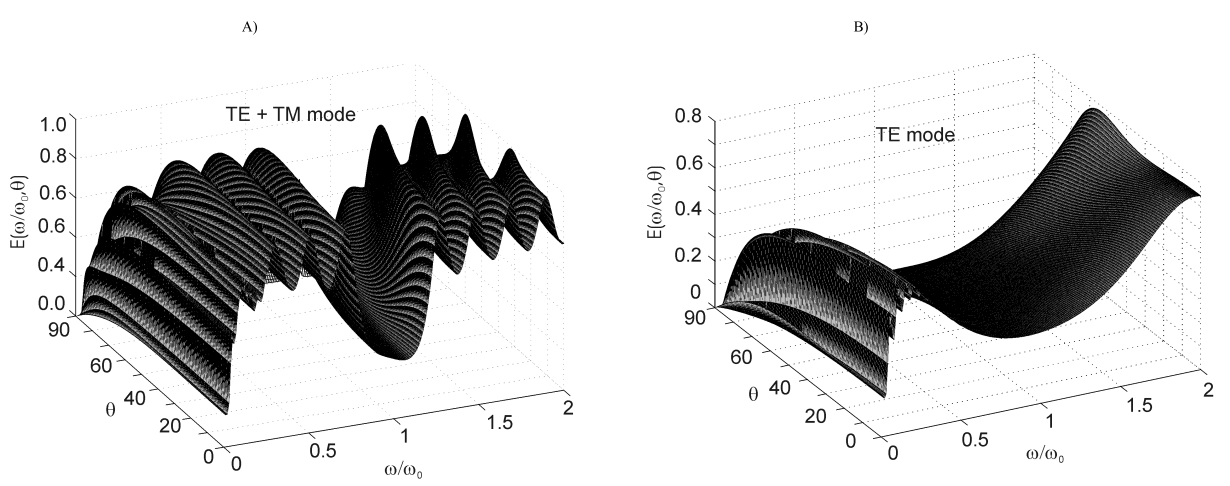

C)
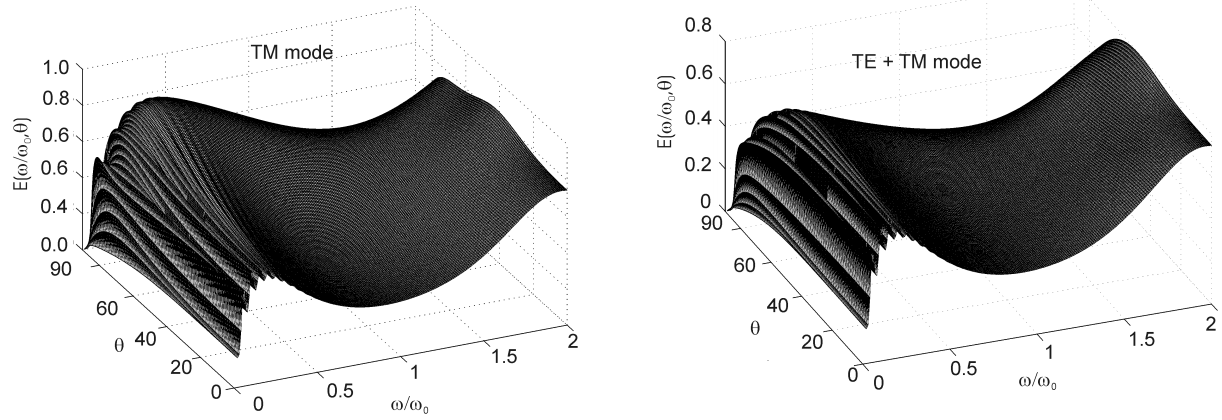

Figure 7.2: Emittance as a function of incident angle $\theta$ and normalized frequency $\omega / \omega_{0}$ for NIM-containing $1 D$ PBG structure, 5 periods, $n_{A}=1.41, n_{B}=-2$; a) unpolarized case, positive index material, $n_{A}=1.41, n_{B}=2$. b) TE-mode polarized emittance $E_{T E}$; c) TM-mode polarized emittance $E_{T M}$; d) unpolarized $E=1 / 2\left(E_{T E}+E_{T M}\right) ;$ In all cases $n_{S}=3+i 0.3$ for the substrate.

no sharp frequency shifts between polarizations. Such behavior is a result of phase compensation, see Chapter 5 and references therein.

Further we considered a more realistic case with dispersion taken into account. We used the form (7.2), assuming that $\Gamma=0$. Figure 7.3 depicts the emittance versus incident angle $\theta$ and normalized frequency $\omega / \omega_{0}$ modified by dispersive NIM for unpolarized case. We used the Drude model for the description of metamaterials [31]. The dispersion we used was $n_{B}\left(\omega / \omega_{0}\right)=1-3\left(\omega / \omega_{0}\right)^{2}$ where $\omega_{0}$ is the quarter-wavelength frequency. We chose $\omega_{p}=\omega_{0} / \sqrt{3}$ in (7.2), thus obtaining $n_{B}=-2$ at $\omega / \omega_{0}=1$, the same absolute refractive index value as that used to calculate the dispersionless case in Figure 7.2. We further used $n_{A}=2$, $L_{A}=0.25 \lambda_{0} / n_{A}, L_{B}=0.25 \lambda_{0} / n_{B}$ and $L_{S}=10 \lambda_{0} / \operatorname{Re}\left(n_{S}\right)$. The calculated emittance in Figure 7.3 clearly shows the existence of full phase compensation in the emittance spectrum (the peak values at the frequency $\omega / \omega_{0}=1$.

Similar to zero-n photonic band gap [46, 110], also obtained by stacking alternating layers of positive index materials and NIM but furnishing transmission 


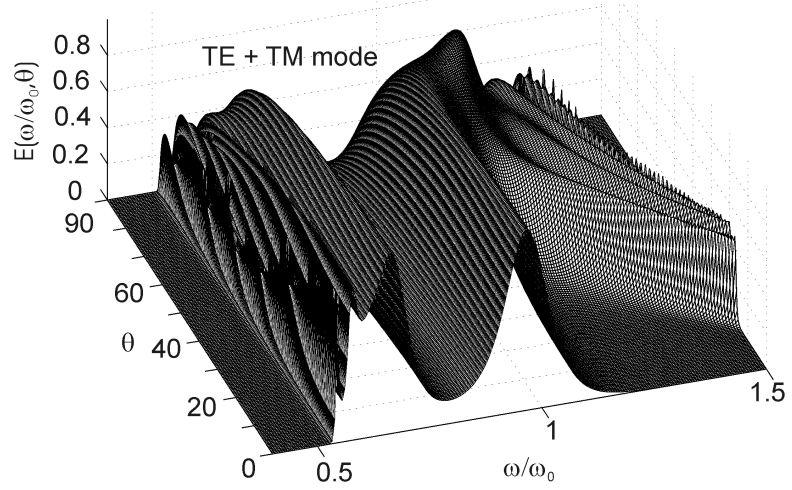

Figure 7.3: Emittance $E\left(\omega / \omega_{0}, \theta\right)$ as a function of incident angle $\theta$ and normalized frequency $\omega \omega_{0}$ for unpolarized case with dispersion taken into account, $n B\left(\omega / \omega_{0}\right)=1-3\left(\omega_{0} / \omega\right)^{2}$ and $\omega_{0}=2 \pi c / \lambda_{0}$-quarter-wavelength frequency (at this frequency $n_{B}=-2$ ) $n_{A}=2, L_{A}=0.25 \lambda_{0} / n_{A}, L_{B}=0.25 \lambda_{0} / n_{B}$, $L_{S}=10 \lambda_{0} / \operatorname{Re}\left(n_{S}\right)$

minimum, this phase compensated situation arises when the averaged effective refractive index of the structure equals zero. The resulting narrow transmission peak is almost invariant with respect to a length scale change and almost insensitive to angular dependence [45]. There is a shift toward higher frequencies in spectral emittance for phase compensated situation in for larger angles. The same feature can be observed both in periodic and in quasi-periodic spectra [118].

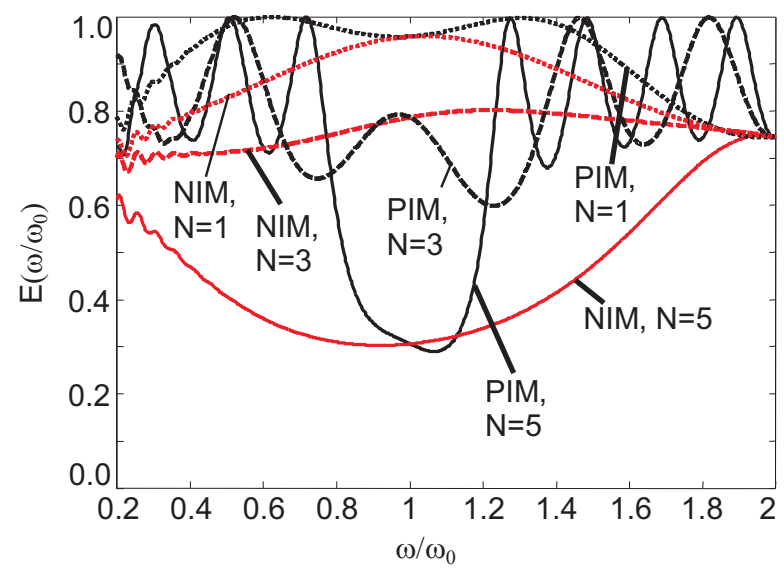

Figure 7.4: Comparison of emittance for conventional multilayers and NIM for different numbers of layer pairs $N, n_{A}=1.41, n_{S}=3+i 0.3 . n_{B}=-2$ for NIM case and $n_{B}=2$ for conventional multilayer case.

Figure 7.4 show the changes of the spectral emittance with an increase of layer 
pairs number for dispersionless NIM-containing multilayers with absorptive substrate for the case of normal incidence. A comparison to the case of positive index multilayer material is given. While the spectrum of the positive material becomes progressively more complex with a layer number increase, the NIM-containing multilayers dependence remains more flat, while the suppressed range is wider. The observed spectral behavior of the NIM-containing structures is a consequence of marked phase compensation. It suppresses the influence of multiple reflections between individual layers and even completely removes it at a single frequency corresponding to the fully compensated structure (zero phase shift). This results in much less pronounced side ripples in spectral characteristics.

An increase of the number of layers spreads the band of suppressed emittance instead of magnifying and multiplying the ripples like in positive index material case. Generally, the interference spectra which are characteristic for the all-PIM structures are flattened and spread due to phase compensation effects. This behavior is observed in NIM-containing multilayers regardless of the fact if dispersion is taken into account or not, since phase compensation occurs in both cases.

Another situation of interest for thermal radiation modification are non-periodic filter geometries [119, 117], [118]. Their band structure are more complex compared to periodic ones, which results in appearance of sharp resonance peaks [16]. Similar to periodic structures, non-periodic NIM multilayers also exhibit a strong influence of phase compensation to transmission [122],[47, 48]. Spectral selfsimilarity and narrow resonance spectral peaks occurs, which has an applicative potential itself. Also, defect based periodic multilayers with NIM may be used for emittannce tailoring [120].

\subsection{Concluding remarks}

We analyzed modification of Planck's blackbody spectra by periodic structures incorporating NIM. Similar to positive-index photonic crystals to which such structures are related, they can be used to enhance, suppress or attenuate spontaneous emission in all or certain directions by changing the density of modes. The paper handles the case of finite structures. Our results show that structures containing NIM show larger influence to the thermal radiation spectrum than all-dielectric PBGs. The suppressed region of thermal radiation is wider, and the spectral characteristics more flat, i.e. without sharp oscillation typical for the all-dielectric case. It can be also seen that spectral properties of the NIM-containing 1D structures are less dependent on the angle of incident radiation. The procedure presented here is of interest in designing other types of non-periodic NIM multilayers for emittance tailoring. The described approach, in principle, can be generalized to $2 \mathrm{D}$ and 3D PBG materials incorporating NIM media. 
Chapter 7. Thermal radiation and...

7.5. Concluding remarks 


\title{
Chapter 8
}

\section{Emittance tailoring by Cantor multilayers containing negative index metamaterial}

\begin{abstract}
$^{1}$
We investigate electromagnetic wave propagation through one-dimensional stacks of alternating positive and negative refractive index layers arranged as truncated pre-fractal Cantor multilayers. We utilized the transfer matrix technique and applied the Kirchhoffs second law to calculate emittance and absorptance modification of thick substrate by negative index metamaterial Cantor multilayers. We took into account dispersion and absorptive losses and analyzed both on-axis and off-axis radiation. We showed that Cantor multilayers formed by inserting negative refractive index layers as a substitution part in the multilayer lattices enable tailoring of both spectral and angular dependencies of emittancelabsorptance.
\end{abstract}

\footnotetext{
${ }^{1}$ This chapter is adapted from: M. Maksimovic, Z. Jaksic, Emittance and absorptance tailoring by negative refractive index metamaterial-based Cantor multilayers, J. Optics A: Pure and Applied Optics , 8 ,3,pp. 355-362, 2006
} 


\subsection{Introduction}

The artificial composite electromagnetic structures with simultaneously negative permittivity and permeability are variously dubbed negative index metamaterials (NIM), left-handed metamaterials (LHM), negative phase velocity media [31, 30, 97], etc. Starting from the Maxwell equations with the appropriate boundary and initial conditions and utilizing the general principles of energy conservation and causality it can be shown that the refractive index of media with negative permittivity and permeability is also negative $[30,32]$. This results in various peculiar properties of the NIM , e.g. the reversal of the Snell's law or the negative refraction, reversal of Doppler shift and Cerenkov radiation, etc. In contrast to the positive index media (PIM), in NIM the light propagates in the opposite direction to the energy flow and the Poynting vector is anti-parallel to the wave phase velocity vector [30].

A large body of research papers on the NRM has been published in the recent years and many applications have been proposed, one of such being the socalled perfect lenses operating beyond the diffraction limit [99]. In spite of tremendous technological difficulties to fabricate shorter-wavelength NRM, the operating wavelengths have been steadily decreasing in recent years and negative refractive behaviour has been observed in the optical range [39, 40].

One of the topics of interest in optics of NIM is the distribution of electromagnetic modes in NIM-containing multilayers. The role of conventional PIM (positive index material) photonic crystals in modifying thermal radiation was researched first by Cornelius and Dowling [52], then by different teams [127, 53, 54, 55, 133]. The influence of NRM to thermal radiation distribution was investigated in [116, 117, 119],[118],[120]. Important differences in comparison to the all-PIM case occur as a consequence of the phase compensation, a phenomenon arising in NIM and resulting in a partial or full removal of phase shift of an electromagnetic wave propagating through a PIM-NIM structure, see Chapter 5 and references therein. We considered the modification of thermal radiation by periodical structures containing NIM in [116]. We showed that NIM-containing structures can be used to enhance or suppress thermal radiation spectrum considerably more than all-positive PBGs. The suppressed region of thermal radiation is wider, the spectral characteristics more flat, and less dependent on the angle of incident radiation. Another type of multilayers with interesting and potentially usable electromagnetic spectral properties are quasi-periodic structures and fractal multilayers [16, 15, 134, 135]. Due to their structural self-similarity, these show spectral regularities in their transmission/reflection, including spectral scalability (the whole spectrum of a given generation repeats scaled as a part of the next generation spectrum) and sequential splitting (spectral peaks from one generation split into doublets in the next) [136]. The strong resonances in spectral dependences of fractal multilayers can localize light very effectively. Thus they were proposed for different applications, including very narrow pass and band reject filters. The possibility to produce multilayers combining fractality and NIM materials (where both of these could induce 
new and interesting phenomena) naturally attracted the attention of researchers. NIM metamaterial-containing Cantor sets were analyzed in [122] for the lossless and dispersionless case. Zero-n photonic band gap in Fibonacci stacks containing NIM were investigated in [121]. In this paper we analyze the influence of multilayer filters based on Cantor sets to the thermal radiation distribution and analyse its applicability to absorptance-emittance tailoring $[117,118]$. In our calculations we utilize Kirchhoff's law and apply the transfer matrix method, at the same time taking into account absorption losses and refractive index dispersion.

\subsection{Theory}

Several classes of fractal (quasiperiodic or non-periodic deterministic) structures distinguish themselves in dependence on the algorithm used for the stack construction. The first class, called the substitutional lattices, is 'generated' via a repeated substitution rule. Such structures(Cantor and Fibonacci multilayers and others) have self-similar optical transmission spectra, and the frequencies of resonance peaks form a fractal set $[16,15,134,135]$.

The second large class represents multilayers that are fractal by themselves. They are called multilayer fractal structures because they are constructed according to a known fractal generation algorithm. This algorithm has to be stopped at some point in order to get a finite structure. Any structure obtained in this way is not a genuine fractal, but rather a prefractal $[18,137,17]$.

Fractal structures were widely studied in conjunction with electronic properties of quasi-crystals, superlattices and optical multilayers [123, 16, 15, 134, 135]. We proceed in applying such formal schemes to obtain spectral features describing the influence of phase compensation arising from negative refractive index layers.

In this paper we restrict ourselves to the simplest form of the so-called 'triadic' Cantor multilayer [136],[138]. We define two refractive indices $\left(n_{A}, n_{B}\right)$ and geometrical thickness values $\left(d_{A}, d_{B}\right)$ that correspond to two materials (A) and (B). The Cantor 'triadic' sequence is defined by the rule

$$
S_{n}=S_{n-1} B_{n} S_{n-1} \text { for } n \geq 2, \quad S_{0}=A, S_{1}=A B A
$$

where $B_{n}$ for the n-th layer denotes the block B with a thickness of $d_{B_{n}}=3^{n-1} d_{B}$. Thus a triadic Cantor set is formed by splitting an interval in material A (let us denote it as $[0,1]$ ) into 3 pieces. The center piece (from $1 / 3$ to $2 / 3$ ) is replaced by material $\mathrm{B}$. Then each of the remaining intervals of $\mathrm{A}([0,1 / 3]$ and $[2 / 3,1])$ is split into three and the process is repeated from the beginning. Theoretically, for a full fractal set the division should continue infinitely long, but in reality a truncated set is retained (a pre-fractal set). One of the reasons is that a Cantor structure with a very large number of layers would have a strongly decreased transmissivity and thus would become useless from the practical point of view [139].

A non-periodic multilayer can be in-bound (with the total thickness of the structure given at the beginning, while one performs its subdivision to develop 
higher generations) or out-bound (single layer thickness is given, one stacks such strata according to a sequence rule to develop higher generations) [15].

Throughout this paper we used the out-bound form, since it is the most practical for NIM-containing structures (NIM itself has to assume a fine structure which is much larger than the atom scale and thus poses a severe limit to in-bound division). Moreover, such an approach enables a direct comparison of transmission optical spectra for different values of $\mathrm{N}$.

We further define that the constitutive layers have an equal optical thickness, that of a quarter-wavelength slabs $n_{A} d_{A}=n_{B} d_{B}=\lambda_{0} / 4$. Hence, the phase shifts in the corresponding layers are given by

$$
\delta_{A(B)}=\frac{\pi}{2} \Omega \cos \theta_{A(B)}
$$

where $\Lambda=\lambda_{0} / \lambda=\omega_{0} / \omega$ is the normalized frequency and $\theta_{A}, \theta_{B}$ are the angles of propagation for the case of oblique incidence on the multilayer.The structure is deposited on a thick substrate $(d>>\lambda)$ with an index $n_{S}$. We assume that the multilayer is surrounded by a semi-infinite medium $n_{0}$, which in our case for the sake of simplicity is air or vacuum.

A quarter-wavelength optical thickness of the multilayer slabs is chosen to enable easier comparison with prior work, since the same choice is encountered both in literature on absorptance and emittance tailoring by photonic crystals, e.g. in $[134,135]$ and that on NIM $[47,140]$. At the same time, a clear physical picture is obtained without a loss of generality, since other thickness choices would furnish qualitatively similar spectral behavior.

Figure 8.1 shows the construction principle of structures analyzed in this chapter (not in exact scale). Those are a one-dimensional multilayer structure composed of NIM and PIM strata arranged as triadic Cantor sets. Second (G2) and third (G3) Cantor generations for the in-bound case are shown as examples. No plasmon modes in the NRM part interacting with the incident electromagnetic wave were taken into account in this paper. Since the interfaces are assumed to be flat (i.e. no surface roughness, gratings or similar disturb the plasmon modes), no incident propagating plane wave can excite surface plasmons regardless of the incidence angle. The reason is that the plasmon modes must have a larger momentum at the same frequency for all energies considered [31]. Therefore no influence of plasmon modes to the emittance may be expected.

A common practice when studying the spectral properties of optical multilayers is to use the well established numerical technique of the transfer matrix method $[1,4]$, as well as its equivalent counterpart, the recurrence relations for transmission and reflection coefficients [12]. If we assume transfer matrix through a multilayer composed of materials $\mathrm{A}$ and $\mathrm{B}$ in the form of interface matrices $M_{A B}$ (where A,B denote the corresponding $\mathrm{A}$ and B layers) and propagation matrices $M_{p}$ (with $p$ denoting a layer $A(B)$ or $S$ of a given thickness), then we are able to uniquely describe the wave propagation through this multilayer by multiplying these two 


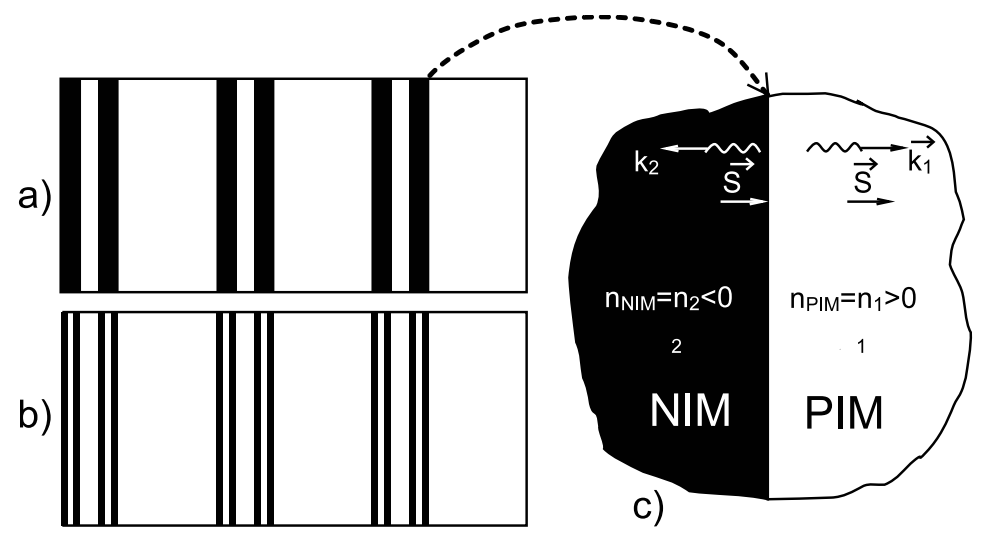

Figure 8.1: A multilyer structure composed of NIM and PIM strata arranged as triadic Cantor sets; a) 2nd generation (G2), b) 3rd generation (G3), c) schematic presentation of a negative index-positive index material interface

matrices.

$$
\begin{gathered}
\mathbf{M}_{A B}=1 / 2\left[1+n_{B} / n_{A} \quad 1-n_{B} / n_{A} ; 1-n_{B} / n_{A} 1+n_{B} / n_{A}\right] \\
\mathbf{M}_{p}=\left[\exp \left(-i \delta_{p}\right) \quad 0 ; 0 \exp \left(+i \delta_{p}\right)\right]
\end{gathered}
$$

In the case of oblique incidence the above matrices retain the same form, but one has to substitute $n_{A(B)} \rightarrow n_{A} \cos \left(\theta_{A(B)}\right)$ for corresponding refractive indices in the propagation matrices for both TE- and TM-polarization, and $n_{A(B)} \rightarrow$ $n_{A(B)} \cos \left(\theta_{A(B)}\right)$ for TE-polarization and $n_{A(B)} \rightarrow n_{A(B)} / \cos \left(\theta_{A(B)}\right)$ for TMpolarization in the interface matrices. The overall transfer matrix for the chosen structure is given as a product of the interface and the propagation matrices [1, 4]. The transmission and the reflection coefficient of the given structure follow from the overall transfer matrix which has the form $\mathbf{M}=\left[\begin{array}{llll}m_{11} & m_{12} ; m_{21} & m_{22}\end{array}\right]$ and are expressed through the formulas

$$
T=\frac{1}{\left|m_{11}\right|^{2}} \text { and } R=\left|\frac{m_{21}}{m_{11}}\right|^{2} .
$$

For our NIM layers we assume a frequency-dependent complex refractive index. In literature on quasi-periodic optical multilayers a common practice is to use frequency independent parameters (e.g. $[134,136])$, but a real NIM metamaterial must be dispersive and lossy in order to preserve causality. We assume that both effective permittivity and permeability posses the same form

$$
\epsilon(\omega)=1-\frac{\omega_{p e}^{2}}{\omega\left(\omega+j \Gamma_{e}\right)}, \quad \mu(\omega)=1-\frac{\omega_{p m}^{2}}{\omega\left(\omega+j \Gamma_{m}\right)}
$$

where $\omega_{p e(m)}$ are the electric (magnetic) artificial plasma frequencies and $\Gamma_{p e(m)}$ are electric (magnetic) damping constants (which can be expressed as a fraction of 
the plasma frequency [31]. We chose $\omega_{p e}=\omega_{p m}=\omega_{p}$ and $\Gamma_{p e}=\Gamma_{p m}=\Gamma_{p}$, thus the refractive index of the NIM part reads

$$
n(\omega)=1-\frac{\omega_{p}^{2}}{\omega\left(\omega+j \Gamma_{p}\right)}
$$

We represented the damping coefficient as a fraction of plasma frequency, which is an often met assumption in literature, acceptable even from the experimental point of view [4],[5].

In our calculations we considered the case when phase compensation [18] occurs. If the wave vector through a generalized PIM-NIM structure is $\mathbf{k}=$ $\mathbf{k}_{0} n_{i}$ (wave number in free space is $k_{0}=2 \pi / \lambda$, and $n_{i}$ is refractive index in material $i(i=A, B)$, then the phase after the end of the second slab is $\phi=$ $k_{A} d_{A}+k_{B} d_{B}=k_{0}\left(n_{A} d_{A}+n_{B} d_{B}\right)$. If the first layer is PIM $\left(n_{A}>0, \mu_{A}=1\right)$ and the second is NIM $\left(n_{B}<0, \mu_{B}=-1\right)$ the total phase difference is $\phi=$ $k_{A} d_{A}+k_{B} d_{B}=k_{0}\left(n_{A} d_{A}-\left|n_{B}\right| d_{B}\right)$. If the appropriate ratio $n_{A} /\left|n_{B}\right|=d_{B} / d_{A}$ is chosen, the total phase difference between the front and the back faces of this two-layer structure becomes exactly zero.

In such a system NIM acts as a phase compensator for both propagating and evanescent waves. It reduces the decay in amplitude of evanescent waves and acts as an amplitude compensator.

If absorption losses are taken into account, the condition for the full phase compensation becomes [107]

$$
\phi=k_{0}\left(n_{A} d_{A}-\left|n_{B}\right| d_{B}\right)+j \kappa_{B} d_{B}
$$

and $\phi=j \kappa_{B} d_{B}\left(\kappa_{B}=k_{0} \operatorname{Im}\left(n_{B}\right)\right)$ for the zero phase difference (i.e. the total phase difference in lossy material is never equal to zero).

Stacking alternating layers of PRM and NRM materials leads to a new type of photonic band gaps with properties very different from those of Bragg gaps, denoted as the zero-n gap. It arises when the averaged effective refractive index of the structure equals zero. It has been demonstrated that the zero-n gap is almost invariant with respect to a (length) scale change, and insensitive to randomness as long as the condition is satisfied [46]. This zero-n gap structure is in fact a fully phase-compensated 1D photonic crystal with impedance mismatch, see chapter 5 .

In the zero-n structures one can observe the existence of discrete modes and photon tunnelling modes in the band structure. The discrete modes can be utilized to make a very narrow filters without side lobes.

All negative-n materials that have been made until now are dispersive, which is in fact a condition imposed by causality [31, 30]. However, by stacking positive$\mathrm{n}$ material and negative-n material in a layered structure, it is always possible to find (within the range of negative refractive index) such frequency that a zero-n condition will be met for some particular frequency [46].

We consider a system in thermal equilibrium at a given temperature, its radiation having a Planck's blackbody spectrum. A non-periodic multilayer filter 
containing NRM material modifies the spectral emissivity of the blackbody radiator and the angular distribution of the radiation. To calculate the modification by the Cantor structures, we determine first the thermal emmittance $\mathrm{E}$ of the multilayer. This is done by an indirect method which makes use of the Kirchhoff's law of detailed balance, (a material's emittance in thermal equilibrium is proportional to its absorptance; for a blackbody they are equal). The absorptance/emittance is defined by the reflection and transmission coefficient of the multilayer as $E=A=1-R-T$, see chapter 5 and references therein.

Once the emittance is obtained, its multiplication by the Planck power spectrum gives the power spectrum of the PBG emitter in terms of its emittance and the blackbody spectrum

$$
\rho^{\text {Cantor }}(\omega)=E^{\text {Cantor }} \rho^{B B}(\omega)
$$

An expression for the Planck radiation law in NRM media was obtained in [128] and in [116].

\subsection{Results and discussion}

We considered PIM-NIM non-periodic Cantor-type structures formed by inserting NIM layers as a substitution part in the multilayer lattices. For comparison, we also analyzed purely positive-material structures (PIM-PIM) with the same geometry. For the most part of this paper we considered lossy and dispersive NIM media.

Figure 8.2 shows as an illustration the results calculated for idealized PIMNIM Cantor structures without losses and dispersion for $n_{A}=1.5, n_{B}=-3$ and $d_{A}=\lambda_{0} / 4 n_{A}, d_{B}=\lambda_{B} / 4 n_{B}$. It can be seen that the transmission spectra exhibit spectral scalability which is the well-known property of conventional positive index (PIM-PIM) structures. Further it is seen that spectral sequential splitting leads to forming of multiple narrow transmission peaks.

One can see that the overall width of the multiplets remains almost unchanged during splitting. This interesting difference of spectral properties between the PIMPIM and the PIM-NIM non-periodic structures is in fact caused by phase compensation that occurs in the second case. Phase compensation in periodic structures causes ripple suppression, transmission spectra flattening and rejection band widening [43]. A similar effect is the cause for distinctive spectral characteristic in NIM-containing fractal multilayer case. It has been suggested that in the case of very large values of $\mathrm{N}$, i.e. an extreme geometrical fractality, the spectral behavior of PIM-NIM structures in general starts to resemble that of the PIM-PIM fractal multilayers [122].

All other calculations in this paper are done for lossy and dispersive NRM where dispersion is taken into account according to (8.7). We chose $n_{B}(\omega)=$ $1-\omega_{p}^{2} / \omega^{2}+j \Gamma \omega_{p}^{2} / \omega^{3}$ with $\omega_{p}=\omega_{0} / \sqrt{3}$ and $\Gamma=0.001 \omega_{p}$ to facilitate calculation and at the same time enable comparison with our previous results [116] without a loss of generality. 

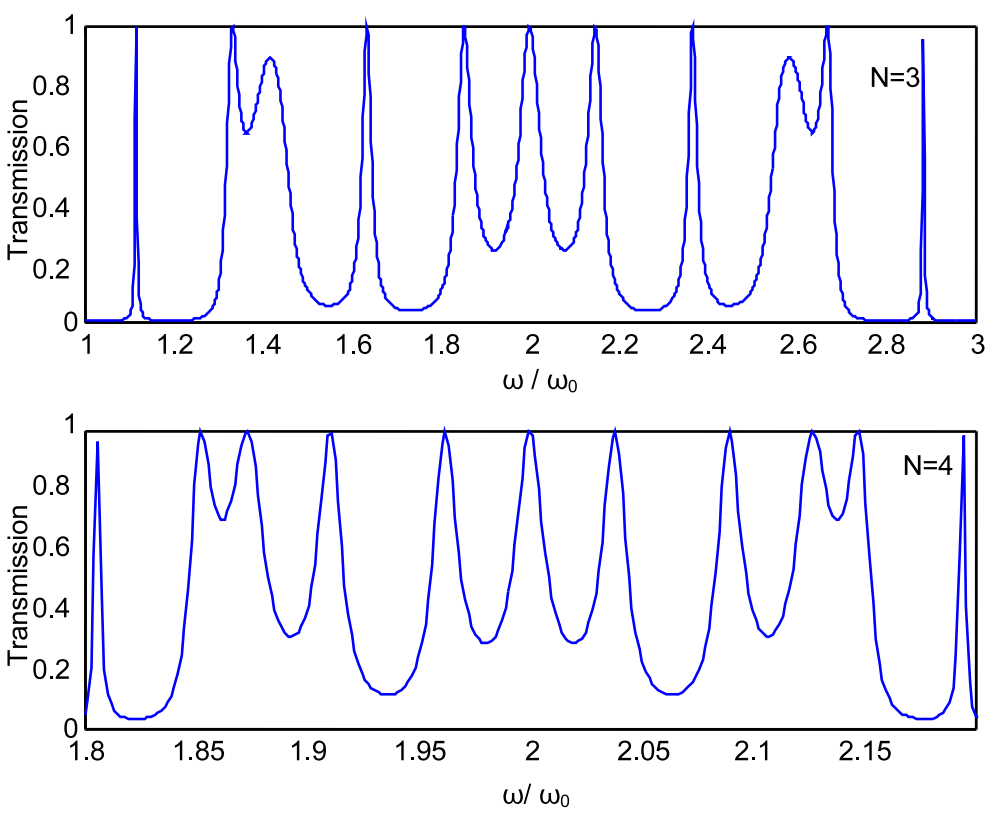

Figure 8.2: Transmission for Cantor $(3,\{1\}, N)$ PRM-NRM multilayer structures: central part of the spectrum for $N=3$ (top) and $N=4$, scaled (bottom) for $n_{A}=1.5, n_{B}=-3$ and $d_{A}=\lambda_{0} / 4 n_{A}, d_{B}=\lambda_{B} / 4 n_{B}$.

Figure 8.3 shows a mean value of the real part of refractive index in the NRMPRM Cantor composite for different generations for the dispersive case. We used the equation [135]

$$
\langle\operatorname{Re}(n)\rangle=\left(N_{j A} n_{A} d_{A}+N_{j B} n_{B} d_{B}\right) /\left(N_{j A} d_{A}+N_{j B} d_{B}\right)
$$

where $j$ is the generation number, $N_{j A}=2^{j}$ and $N_{j B}=3^{j}-2^{j}$ for average refractive index calculation in Cantor multilayer. The dispersion of negative refractive index is shown in the inset of Figure 8.3.

In the case of lossy NIM a true phase compensation does not take place, i.e. an imaginary part of refractive index always exist. However, suppression of the real part of phase shift significantly influences the interference pattern, and if losses are small nearly perfect phase compensation appears. As we regard here lossy and absorptive NRM layers a marked difference can be seen in zero-n band gap condition compared to the lossless case, i.e. no true zero-n band gap appears. This consideration is valid for both periodical and quasi-periodic situations.

Figure 8.4 shows spectral transmission and spectral emittance of a lossy and dispersive structure. The self-similarity of spectrum is mostly removed by dispersion and losses. However, although the dependencies in Figure 8.4 are not strictly self-similar, their patterns reveal a certain degree of fractal-like behavior.

Figure 8.5 shows the spectral absorptance (or emittance) in multilayer structures containing both positive and negative index materials. The suppressed region 


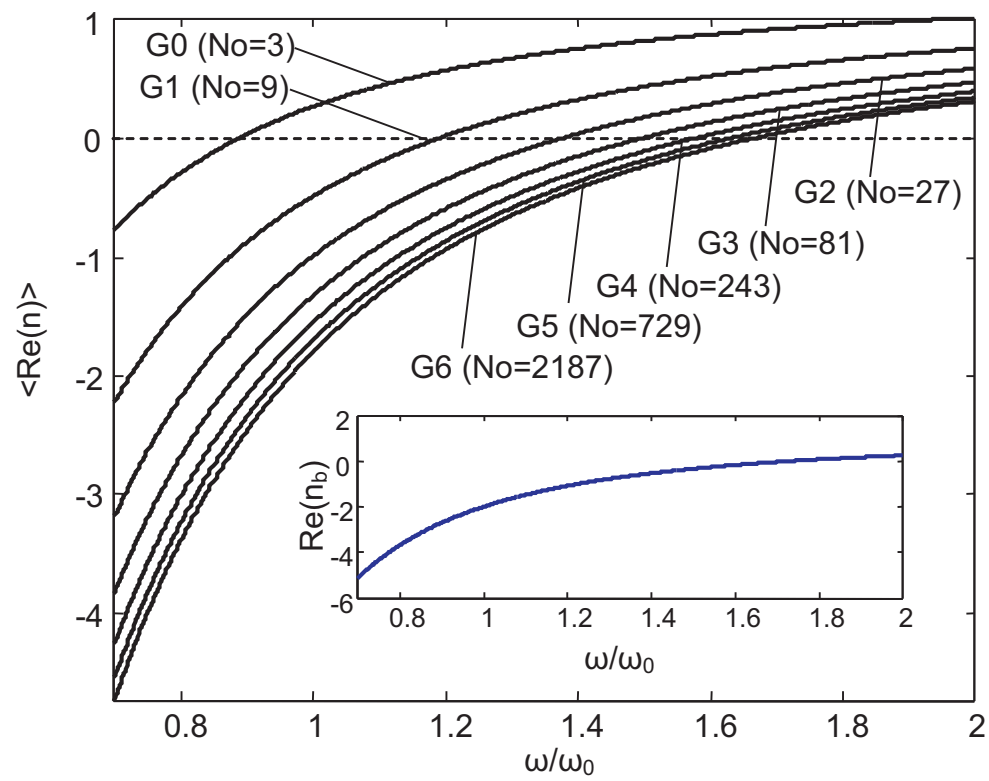

Figure 8.3: Mean value of the real part of refractive index for a Cantor-type composite containing negative index part for different generations (from G0 to G6). The NIM dispersion is shown in the inset.
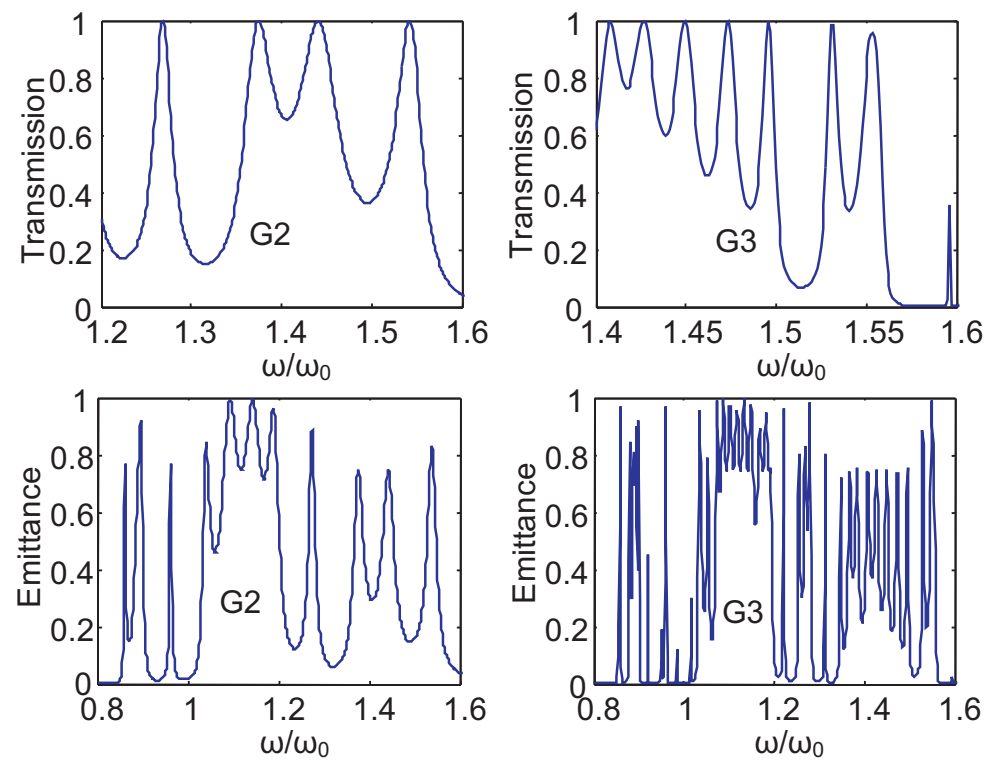

Figure 8.4: Transmission and emittance of a Cantor-type NIM-PIM multilayer for generations $G 2$ and $G 3$. 


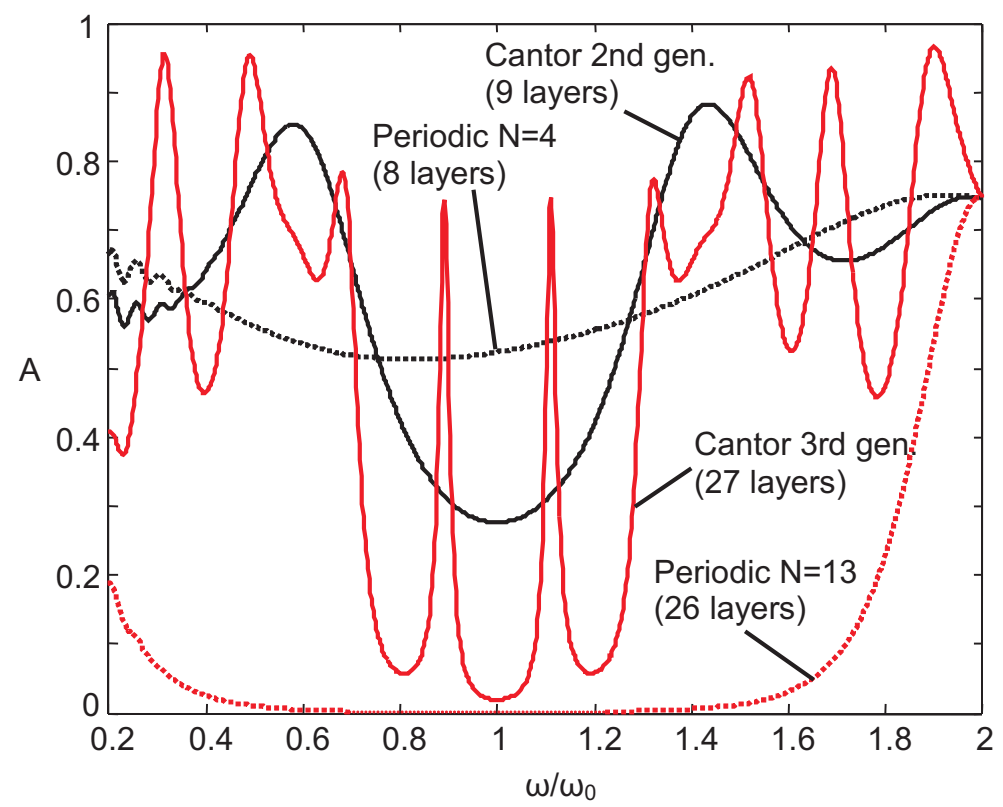

Figure 8.5: Absorptance (or emittance) for periodic versus Cantor-type NIM-PIM multilayers. Structures with similar numbers of separate layers are considered to facilitate comparison. $n_{A}=1.41, n_{B}=-2, n_{S}=3+i 0.3$.

of thermal radiation in periodic NIM-containing structure is wider, and the spectral characteristics flatter (there are no sharp ripples typical for positive index materials) in comparison to the all-positive case. A comparison is given between periodic NIM-containing multilayers and Cantor-type non-periodic structures. A full rejection band appears in spectral absorptance/emittance of periodic structures, whereas Cantor-type pre-fractals exhibits sharp and narrow resonances throughout the band already for such low generation numbers.

Figure 8.8 shows the angular and spectral dependence of emittance of Cantor sets composed of positive refractive index material (PIM-PIM) for generations 0 to 3, and Figure 8 shows the same dependence for the NIM-PIM case. The dependencies were calculated for the TE mode for the dispersionless and lossy case. Similar dependencies are obtained for the TM mode (not shown here).

For higher generation Cantor structures, in the all-positive (PIM-PIM) case a considerable attenuation is caused by a high number of layers, thus they become practically useless for filtering applications [139]. However, due to the effect of phase compensation, in the case of NIM-PIM structures one can use a much larger number of layers (higher generations) in Cantor-type filters and still have a significant transmission and significant absorptance/emittance tailoring effects. This is an important difference in comparison to the conventional all-positive Cantor structures. 

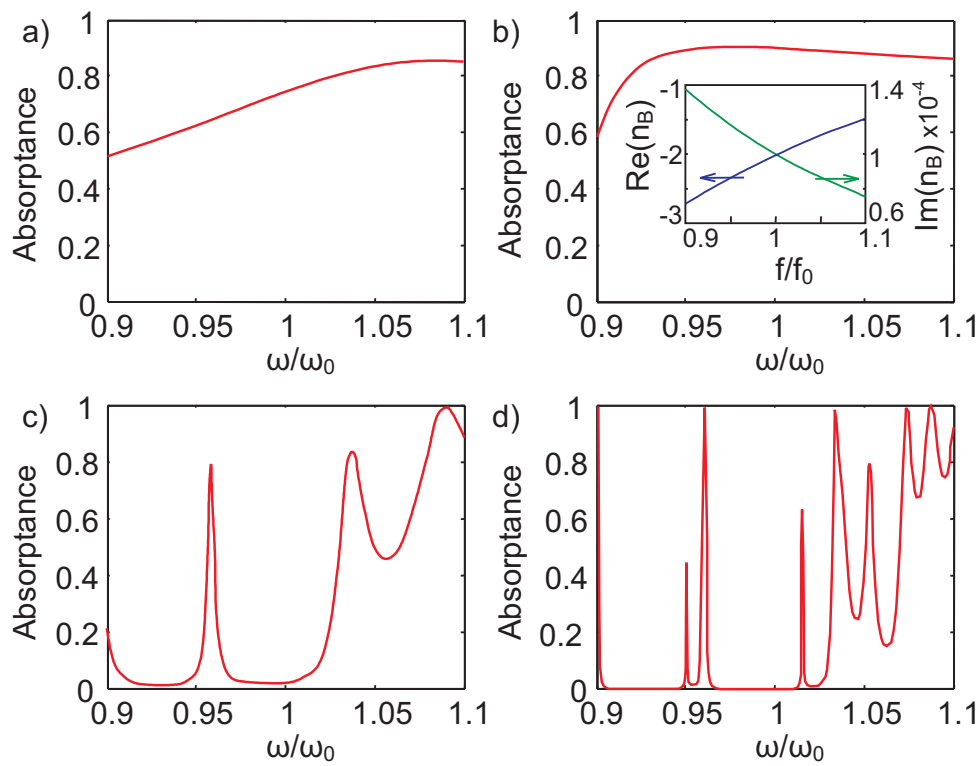

Figure 8.6: Absorptance (or emittance) for four generations of Cantor sets for structures containing dispersive and lossy negative index metamaterial; $n_{A}=1.41$, $n_{S}=3+i 0.3$. Cantor multilayers are a) G0; b) G1; c) G2; d) G3. Dispersion dependence for the metamaterial part $n_{B}$ is shown in inset in Figure 3 (b).

Figure 8.6 shows spectral absorptance for four successive generations ( 0 to 3) of Cantor sets for multilayers containing dispersive and lossy negative index metamaterial with a dispersion dependence as shown in the inset. Although the NIM part is dispersive and lossy, a rich absorptance spectrum is seen already in fourth generation. Figure 8.7 shows the spectral absorptance for higher generations (4 to 8 ), where similar behavior is even more pronounced.

There are several important conclusions to be deduced from the comparison of Figures 7 and 8. First, the influence of the angle of incidence is less pronounced in non-periodic structures containing NIM than in the same structures with all-PIM layers. This may lead to more relaxed conditions when utilizing negative index non-periodic multilayers.

Second, angular and spectral flattening and disappearance of ripples do appear in the NIM-containing structures, but one can still notice a rich spectral behavior which could be useful for practical applications. The flattening is a result of phase compensation which inevitably occurs under certain conditions in NIM containing structures, but in the case of Cantor-type structures this does not remove the useful properties of non-periodic multilayers.

With an increase of the number of layers more peaks usually associated with non-periodic structures appear in the spectral characteristics and the spectral diagrams become more similar to the conventional non-periodic ones, but at the same time the structures remain much less sensitive to incident angle than the all-positive 

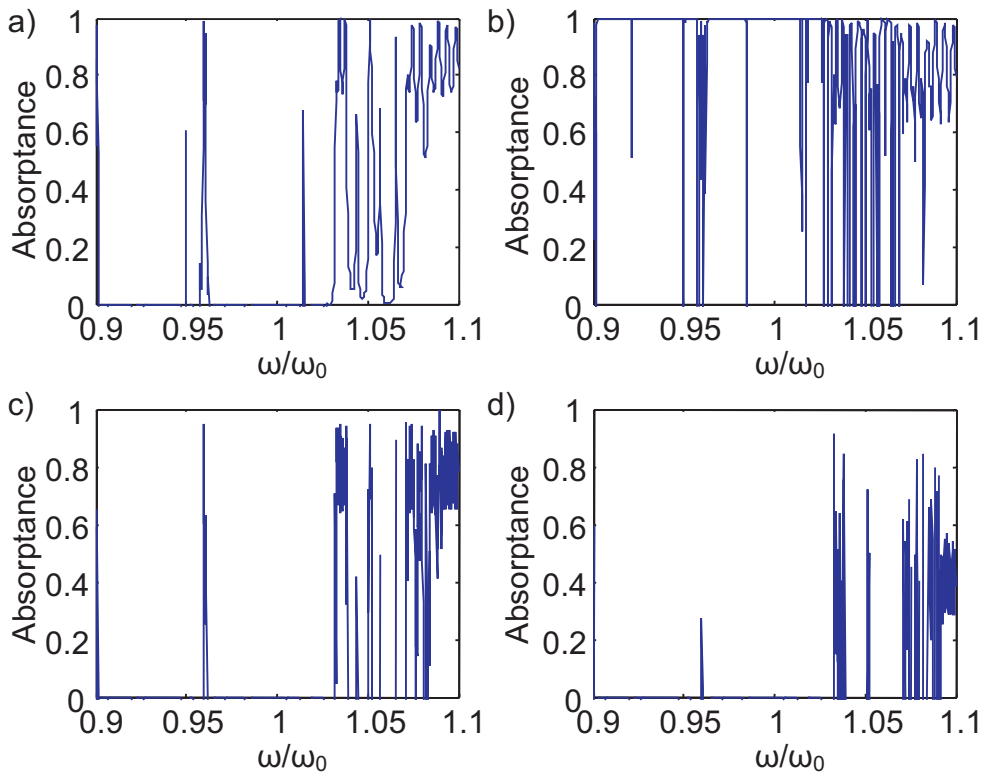

Figure 8.7: Absorptance (or emittance) for four higher generations of Cantor sets for structures containing dispersive and lossy negative index metamaterial; $n_{A}=$ 1.41, $n_{S}=3+i 0.3$. Cantor multilayers are a) $G 4$; b) G5; c) G6; d) $G 7$.

ones.

Figure 8.10 and Figure 8.11 show directional spectral emittance from different generations of Cantor multilayers for frequencies $\omega / \omega_{0}=1$. Figure 8.10 shows the all-positive case. It can be seen that the emittance has a strong angular dependence, while the spatial distribution becomes progressively more complex with an increase of the generation number. Contrary to that, in the NIM-containing Cantor multilayers (shown in Figure 8.11) for lower generations the angular dependence is much weaker than in the all-positive case. For higher generations the directivity of emittance is much more pronounced and it remains at nearly the same angles in different generations.

For generations G0 and G1 it can be seen that the angular dependence of emittance follows a very similar pattern both for NRM-containing and all-positive case. This is consistent with conclusions from [122]. In all polar curves shown in Figure 8.10 and Figure 8.11 one can notice the existence of a stop band (the range of suppressed emittance). In the all-PIM case the band stop quickly disappears with an increased angle, while in the NIM-containing structure it is less sensitive to the propagation angle.

If an additional defect is introduced into the Cantor pre-fractal (not shown here), an adjustment of its parameters allows for an accurate tailoring of angular directivity of emittance (a 'super-directivity generated' tailoring) which could find its practical application. An important conclusion is that NIM-containing Cantor 

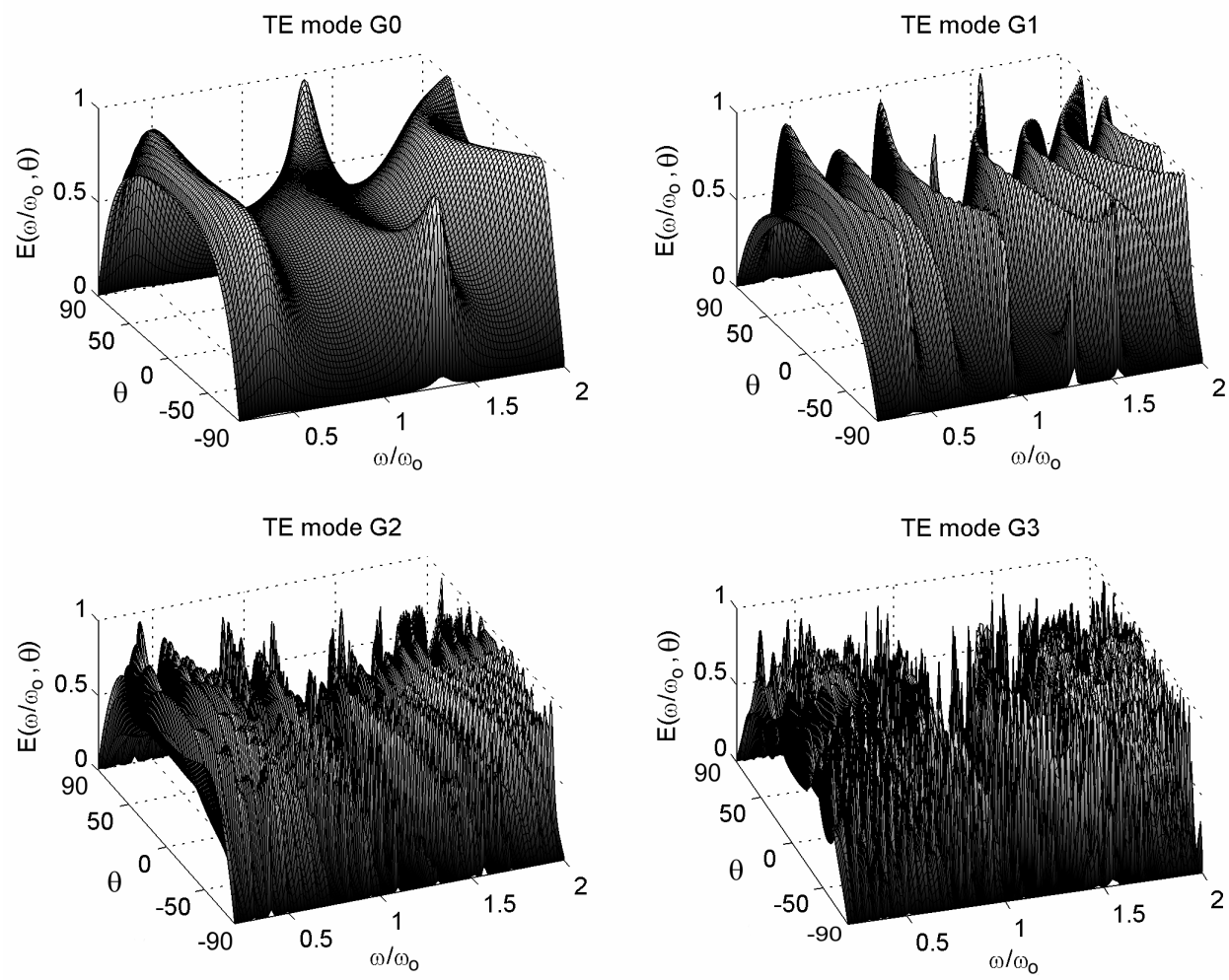

Figure 8.8: Angular and normalized spectral dependence of emittance of Cantor sets composed of positive refractive index materials (PIM-PIM) for generations 0 to 3. $n_{A}=1.41, n_{B}=2, n_{S}=3+i 0.3$.

multilayer enable both spectral and angular tailoring of thermal emittance. This is similar to the periodic case [116].

It is interesting to note another possibility for fractal multilayers designed in metallo-dielectric quasi-periodic stacks [135]. The use of periodic arrangement of metallic and dielectric layers generally leads to a decrease of transmittance with the addition of more layers. However, it is possible to arrange the layers in quasiperiodic geometry in order to maintain high levels of transparency, even when more metal is added to the stack [135]. This is important when considering the 'transparent metal' designs where it is possible to obtain good transparency even in the spectral regions where the intrinsic behavior of metals demands negligible transmission. 

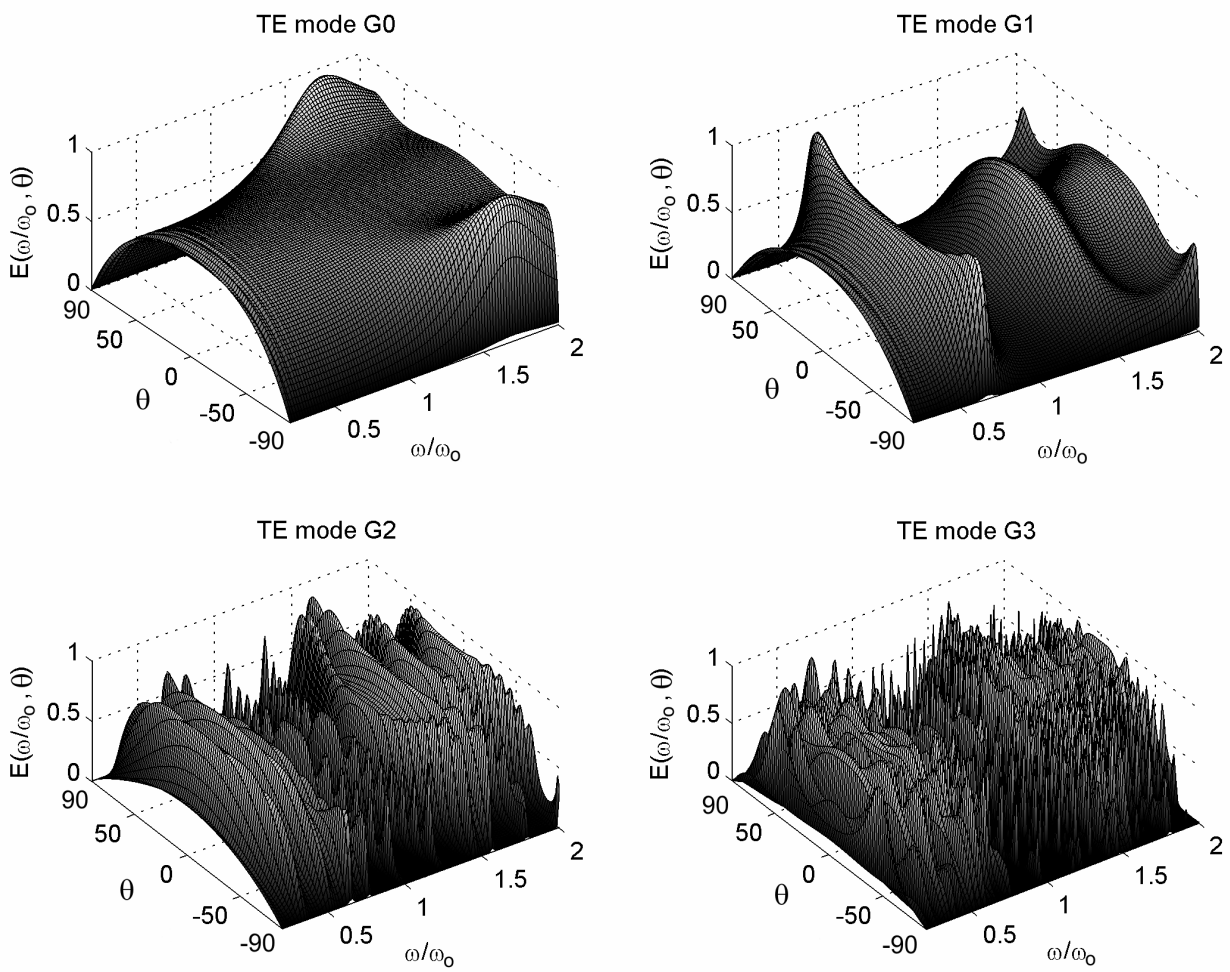

Figure 8.9: Angular and normalized spectral dependence of emittance of Cantor sets composed of positive refractive index materials (PIM-NIM) for generations 0 to 3. $n_{A}=1.41, n_{B}=-2, n_{S}=3+i 0.3$.

\subsection{Concluding remarks}

Our investigations were dedicated to pre-fractal (non-periodic) multilayers of triadic Cantor type containing dispersive and lossy negative refractive index media applied as filtering structures on the top of thick absorbing substrate. We analyzed substrates emittance/absorptance modification caused by such structures. We have found that phase compensation in these structures improves their usability for practical applications compared to the all-PIM case. Also, the dependence of the spectral transmission on waves propagation angle is much weaker than for conventional quasi-periodic filters. A richer spectrum in a relatively narrow band is achieved with a smaller number of layers. Spectral fractal-like behavior occurs for extreme geometrical fractality, but the sequential splitting gives rise to multiple narrow transmission peaks, which has an applicative potential itself. The use of NIM Cantor multilayers enables tailoring of both spectral and angular dependence of the emittance/absorptance (thermal radiation antenna functionality). 

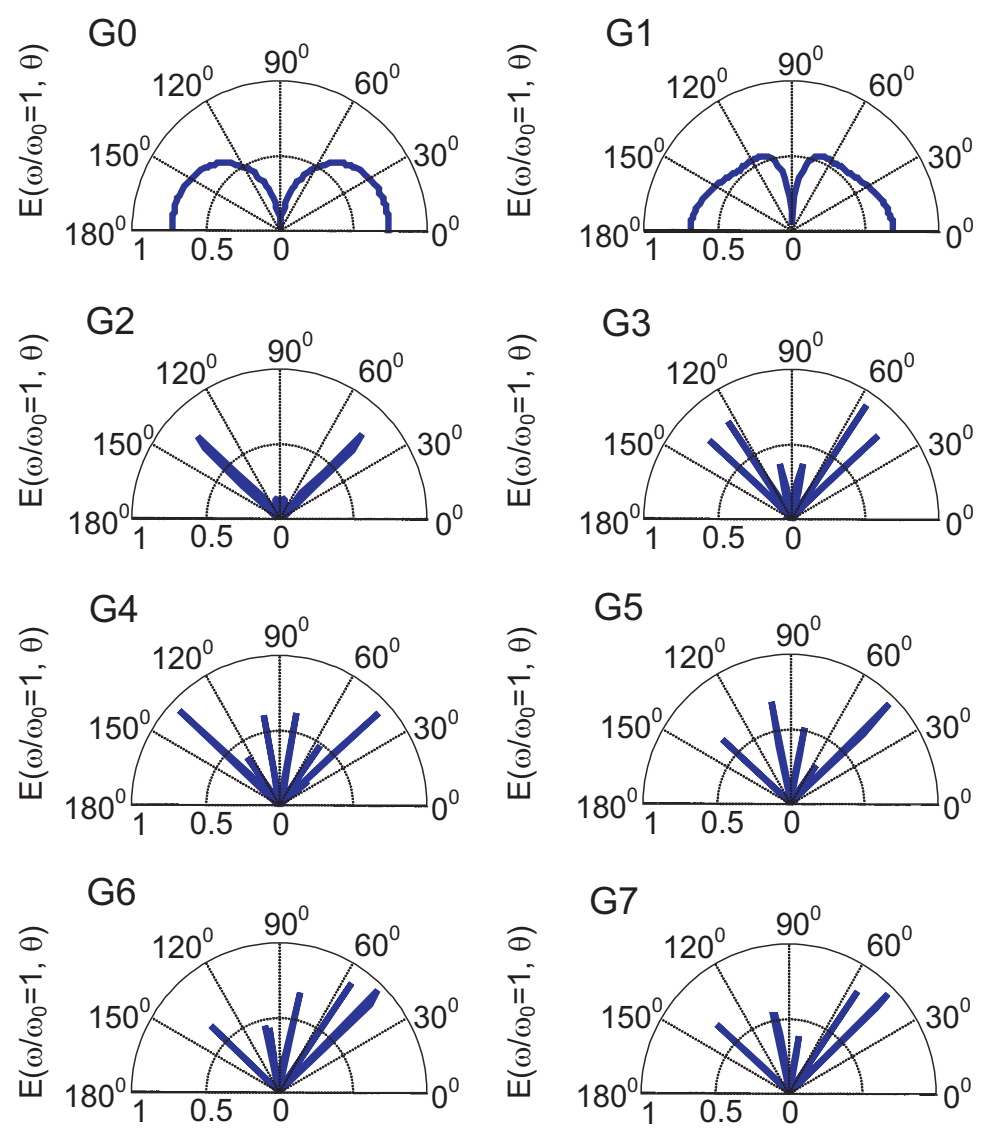

Figure 8.10: Directional spectral emittance for all-positive Cantor multilayers in $T E$ mode for different generations from $G 0$ to $G 7$ and at $\omega / \omega_{0}=1 . n_{A}=1.41$, $n_{B}=2, n_{S}=3+i 0.3$. 

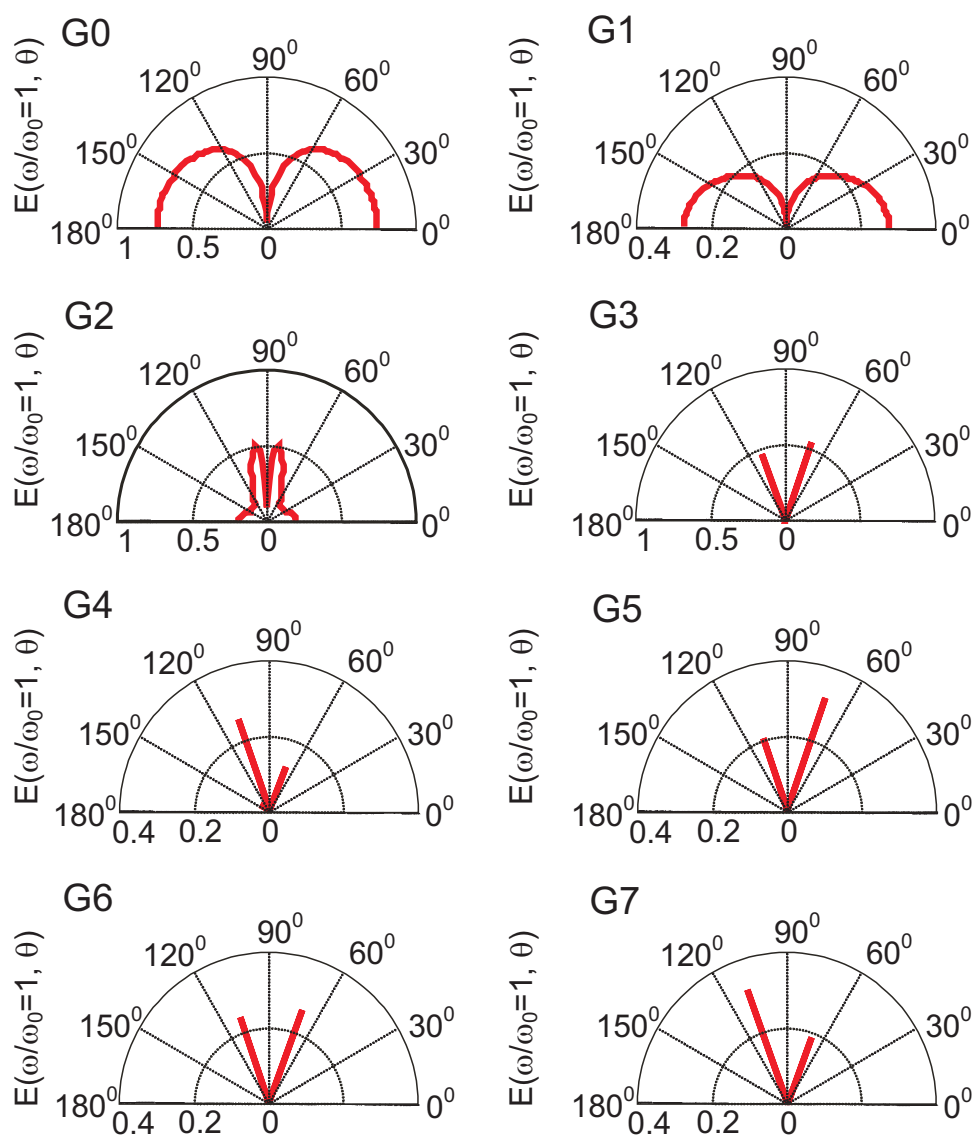

Figure 8.11: Directional spectral emittance for negative refractive index materialcontaining Cantor multilayers in TE mode for different generations from G0 to G7 and at frequency $\omega / \omega_{0}=1 . n_{A}=1.41, n_{B}=-2, n_{S}=3+i 0.3$. 


\section{Bibliography}

[1] M. Born and E. Wolf. Principles of Optics. Cambridge University Press, 2003.

[2] Edward J. Rothwell and Michael J. Cloud. Electromagnetics. CRC Press, 2001.

[3] L. Mandel and E. Wolf. Optical Coherence and Quantum Optics. Cambridge University Press, 1995.

[4] P. Yeh. Optical Waves in Layered Media. Willey, 1988.

[5] H. Angus Macleod. Thin-Film Optical Filters. IOP Publishing, 2001.

[6] C. K. Madsen and J. H. Zhao. Optical Filter Design and Analysis: A Signal Processing Approach. John Willey and Sons, 1999.

[7] J.-M Lourtioz. Photonic Crystals: Towards Nanoscale Photonic Devices. Springer, 2005.

[8] John D. Joannopoulos, Steven G. Johnson, Joshua N. Winn, and Robert D. Meade. Photonic Crystals: Molding the Flow of Light. Princeton University Press, 2008.

[9] K. Sakoda. Optical Properties Of Photonic Crystals. Springer, 2004.

[10] Michael A. Parker. Physics of Optoelelctronics. CRC, Taylor and Francis Group, 2005.

[11] W. T. Silfvast. Laser Fundamentals. Cambridge University Press, 2004.

[12] W. C. Chew. Waves and Fields in Inhomogeneous Media. IEEE Press, 1990.

[13] K. Busch, G. von Freymann, S. Linden, S. F. Mingaleev, L. Tkeshelashvili, and M. Wegener. Periodic nanostructures for photonics. Phys. Rep., 444, 2007.

[14] A. Figotin and A. Klein. Localization of light in lossless inhomogeneous dielectrics. J. Opt. Soc. Am. A, 15:14231435, 1998. 
[15] E.L. Albuquerque and M.G. Cottam. Polaritons in periodic and quasiperiodic structures. Elsevier, 2004.

[16] E.L. Albuquerque and M.G. Cottam. Theory of elemenatary excitations in quasiperiodic structures. Phys. Rep., 376:225-337, 2003.

[17] E. Macia. The role of aperiodic order in science and technology. Rep. Prog. Phys., 69:397-441, 2006.

[18] Aaron D. Jaggard Dwight L. Jaggard and Panayiotis V. Frangos. Fractal Electrodynamics: Surfaces and Superlattices. In Douglas H. Werner and Raj Mittra, editors, Frontiers in Electromagnetics, volume 709, pages 1-47. IEEE Press, 2000.

[19] W. Steurer and D. Sutter-Widmer. Photonic and phononic quasicrystals. J.Phys.D:Appl.Phys., 40:229-247, 2007.

[20] E. S. C. Ching, P. T. Leung, and K. Young. Optical processes in microcavities-The role of quasi-normal modes. In R.K.Chang and A.J.Campillo, editors, Optical processes in mirocavities, chapter 1, pages 1-77. World Scientific, 1996.

[21] M. Zworski. Resonances in physics and geometry. Notices of the AMS, 46(3):319-328, 1999.

[22] S. Hein, T. Hohage, and W. Koch. On resonances in open systems. J. Fluid Mech., 506:255-284, 2004.

[23] R. G. Newton. Scattering Theory of Waves and Particles. Dover Publications Inc., 2002.

[24] E. S. C. Ching, P. T. Leung, A. Maassen van den Brink, W. M. Suen, S. S. Tong, and K. Young. Quasi-normal mode expansion for waves in open systems. Rev. Mod. Phys, 70(4):1545-1554, 1998.

[25] E. W. C. van Groesen and J. Molenaar. Advanced Modeling in Science. SIAM publishers, 2007.

[26] A. Sopahaluwakan. Characterization and simulation of localized states in periodic strucures, PhD Thesis. University of Twente, 2006.

[27] Georg W. Hanson and Alexander B. Yakovlev. Operator Theory for Electromagnetics: An Introduction. Springer, 2002.

[28] Frederick W. Byron Jr. and Robert. W. Fuller. Mathematics of Classical and Quantum Physics. Dover Publications Inc., 1992.

[29] Tosio Kato. Perturbation Theory for Linear Operators. Springer, 1995. 
[30] P. W. Milonni. Fast Light, Slow Light and Left-Handed Light. IOP Publishing, 2005.

[31] S. A. Ramakrishna. Physics of negative refractive index materials. Rep. Prog. Phys., 68:449-521, Jun 2005.

[32] V. G. Veselago. The electrodynamics of substances with simultaneously negative values of epsilon and mu. Sov. Phys. Uspekhi, 10(509), 1968.

[33] N. Engheta and R. W. Ziolkowski (editors). Metamaterials: Physics and Engineering Explorations,. Wiley-IEEE Press, 2006.

[34] D. R. Smith and J. B. Pendry. Homogenization of metamaterials by field averaging. J. Opt. Soc. Am. B, 23:321, 2006.

[35] R. Liu, T. J. Cui, D. Huang, B. Zhao, and D. R. Smith. Description and explanation of electromagnetic behaviors in artificial metamaterials based on effective medium theory. Phys. Rev. E, 76:026606, 2007.

[36] Sergei Tretyakov. Analytical Modeling in Applied Electromagnetics. Norwood, MA: Artech House, 2003.

[37] C. Caloz and T. Itoh. Elelctromagnetic Metamaterials: Transmission Line Theory and Microwave Applications. John Willey and Sons, Inc., 2006.

[38] Z. Jaksic, N. Dalarsson, and M. Maksimovic. Negative Refractive Index Metamaterials: Principles and Applications. Microwave Review, 12(1):3649, 2006.

[39] V.M. Shalaev, W. Cai, U.K. Chettiar, H.-K. Yuan, A.K. Sarychev, V.P. Drachev, and A.V. Kildishev. Negative index of refraction in optical metamaterials. Opt. Lett., 30(24):3356, 2005.

[40] V.M. Shalaev. Optical negative-index metamaterials. Nature Photonics, $1: 49-55,2006$.

[41] T.A. Klar, A. V. Kildishev, V. P. Drachev, and V. M. Shalaev. NegativeIndex Metamaterials: Going Optical. IEEE J. Selec. Top. in Quant. El., 12(6):1106-1115, 2006.

[42] I.S. Nefedov and S.A. Tretyakov. Photonic band gap structure containing metamaterial with negative permittivity and permeability. Phys. Rev. E, 66:036611, 2002.

[43] H. Cory and C. Zach. Wave propagation in metamaterial multi-layered structures. Microwave and Opt. Tech. Lett., 40(6), 2004.

[44] I. V. Shadrivov, A. A. Sukhorukov, and Yu. S. Kivshar. Complete band gaps in one-dimensional left-handed periodic structures. Phys. Rev. Lett., 95:193903-4, 2005. 
[45] I. V. Shadrivov, N. A. Zharova, A. A. Zharov, , and Yu. S. Kivshar. Defect modes and transmission properties of left-handed bandgap structures. Phys. Rev. E, 70:046615-6, 2004.

[46] J. Li, L. Zhou, C. T. Chan, and P. Sheng. Photonic band gap from a stack of positive and negative index materials. Phys. Rev. Lett., 90(083901-1), 2003.

[47] F. F. de Medeiros, E. L. Albuquerque, and M. S. Vasconcelos. Optical transmission spectra in quasiperiodic multilayered photonic structure. J. Phys.: Condens. Matter, 18:8737-8747, 2006.

[48] J.A. Monsoriu, R. A. Depine, and E. Silvestre. Non-Bragg band gaps in 1d metamaterial aperiodic multilayers. J. Eur. Opt. Soc., 2(07002), 2007.

[49] R. Siegel and J. R. Howell. Thermal Radiation Heat Transfer. McGrawHill, Inc, 1972.

[50] Michael F. Modest. Radiative Heat Transfer. Academic Press, 2003.

[51] B. E. A. Saleh and M. C. Teich. Fundamentals of Photonics. John Wiley and Sons, Inc., 1991.

[52] Christopher M. Cornelius and Jonathan P. Dowling. Modification of Planck blackbody radiation by photonic band-gap structures. Phys. Rev. A, 59(6):4736-4746, 1999.

[53] A. Narayanaswamy and G. Chen. Thermal emission control with onedimensional metallodielectric photonic crystals. Phys. Rev. B, 70(125101), 2004.

[54] F. OSullivan, I. Celanovic, N. Jovanovic, J Kassakian, S Akiyama, and K. Wada. Optical characteristics of one-dimensional $\mathrm{Si} / \mathrm{SiO} 2$ photonic crystals for thermophotovoltaic applications. J. Appl. Phys., 97(033529), 2005.

[55] M. Florescu, H. Lee, A. J. Stimpson, and J. P. Dowling. Thermal emission and absorption of radiation in finite inverted-opal photonic crystals. Phys. Rev. A, 72(033821), 2005.

[56] M. Florescu, K. Busch, and J. Dowling. Thermal radiation in photonic crystals. Phys. Rev. B, 75:201101(R), 2007.

[57] B. Hoenders. Completeness of a set of modes connected with the electromagnetic field of a homogeneous sphere embedded in an infinite medium. J.Mat.Phys., 11(9):1815-1832, 1978.

[58] S. M. Dutra and G. Nienhuis. Quantized mode of a leaky cavity. Phys. Rev. A, 62:063805-1-13, 2000. 
[59] Frank Ihlenburg. Finite Elements Analysis of Acoustic Scattering. SpringerVerlag New York, Inc., 1998.

[60] J. Berenger. A perfectly matched layer for the absorption of electromagnetic waves. J. Comput. Phys., 114:185200, 1994.

[61] J.H. Mathews and K.D. Fink. Numerical Methods Using MATLAB. Prentice Hall, 1999.

[62] Donald G. Dudley. Mathematical Foundations for Electromagnetic Theory. IEEE Press, 1994.

[63] Ward Cheney. Analysis for Applied Mathematics. Springer-Verlag New York, Inc., 2001.

[64] Lawrence C. Ewans. Partial Differential Equations. American Mathematical Society, 1997.

[65] A. Settimi, S. Severini, N. Mattiucci, C. Sibilia, M. Centini, G. D’Aguanno, M. Bertolotti, M. Scalora, M. Bloemer, and C. M. Bowden. Quasi-normal mode description of waves in one-dimensional photonic crystals. Phys. Rev E, 68:026614/1-026614/11, 2003.

[66] D. Felbacq. Poles and zeros of the scattering matrix associated with defect modes. J. Phys. A: Math. Gen., 33:7137-7141, 2000.

[67] F. Barra and P. Gaspard. Scattering in periodic systems: from resonances to band structure. J. Phys. A: Math. Gen., 32:3357-3375, 1999.

[68] P. T. Leung, S. Y. Liu, and K. Young. Completeness and orthogonality of quasinormal modes in leaky optical cavities. Phys. Rev. A, 49(4):3057-3067, 1994.

[69] P. T. Leung, S. S. Tong, and K. Young. Two-component eigenfunction expansion for open systems described by wave equation I: completeness of expansion. J.Phys. A: Math.Gen., 30:2139-2151, 1997.

[70] P. T. Leung, S. S. Tong, and K. Young. Two-component eigenfunction expansion for open systems described by wave equation II: linear space structure. J.Phys. A: Math.Gen., 30:2153-2162, 1997.

[71] S. Severini, A. Settimi, C. Sibilia, M. Bertolotti, A. Napoli, and A. Messina. Second quantization and atomic spontaneous emission inside one-dimensional photonic crystals via a quasi-normal mode approach. Phys. Rev. E, 70:056614-1-11, 2004.

[72] M. Bertolotti. Linear one-dimensional resonant cavities. In F.Michelotti, A.Driessen, and M.Bertolotti, editors, Microresonators as building blocks 
for VLSI photonics, volume 709 of AIP Conference Proceedings, pages 1947. AIP, 2004.

[73] K. C. Ho, P. T. Leung, Alec Maassen van den Brink, and K. Young. Second quantization of open systems using quasi normal modes. Phys. Rev. E, 58(3):2965-2978, 1998.

[74] P. T. Leung, W. M. Suen, C. P. Sun, and K. Young. Waves in open systems via a biorthogonal basis. Phys. Rev. E, 57(5):6101-6104, 1998.

[75] Alec Maassen van den Brink and K. Young. Jordan blocks and generalized bi-orthogonal bases: realizations in open wave systems. J. Phys. A: Math. Gen., 34:2607-2624, 2001.

[76] B. Hoenders. On the completeness of the natural modes for quantum mechanical potential scattering. J.Mat.Phys., 20:329-335, 1979.

[77] B. Hoenders and M. Bertolotti. The quasi normal mode description of the scattering process by dispersive photonic crystals. Proc. SPIE, 6182:61821F1-9, 2006.

[78] S. H. Tang and M. Zworski. Resonance expansions of scattered waves. Comm. Pure Appl. Math, 53:1305-1334, 2000.

[79] S. Severini, A. Settimi, C. Sibilia, M. Bertolotti, A. Napoli, and A. Messina. Quasi-normal frequencies in open cavities: An application to photonic crystals. Acta Phys. Hung. B, 23/3-4:135-142, 2005.

[80] S. Severini, A. Settimi, C. Sibilia, M. Bertolotti, A. Napoli, and A. Messina. Quantum counter-propagation in open optical cavities via the quasi-normalmode approach. J. Laser Phys., 16/6:911-920, 2006.

[81] D. Felbacq and R. Smaâli. Density of states for finite photonic crystals. Phys. Rev. B, 67:085105, 2003.

[82] D. Felbacq. Numerical computation of resonance poles in scattering theory. Phys. Rev. E, 64:047702, 2001.

[83] E. Anemogiannis, E. N. Glytsis, and T. K. Gaylord. Determination of Guided and Leaky Modes in Lossless and Lossy Planar Multilayer Optical Waveguides: Reflection Pole Method and Wavevector Density Method. J. Light. Tech, 17(5):929-941, 1999.

[84] C. Vasallo. Optical Waveguide Concepts. Elsevier, 1991.

[85] M. Hammer. Hybrid analytical/numerical coupled-mode modeling of guided wave devices. J. of Light. Tech., 25(9):2287-2298, 2007. 
[86] M. Bayindir, C. Kural, and E. Ozbay. Coupled optical microcavities in onedimensional photonic bandgap structures. J. Opt. A: Pure and App. Opt., 3:184-189, 2001.

[87] Sheng Lan, Satoshi Nishikawa, Yoshimasa Sugimoto, Naoki Ikeda, Kiyoshi Asakawa, and Hiroshi Ishikawa. Analysis of defect coupling in one- and two-dimensional photonic crystals. Phys. Rev. B, 65(16):165208, 2002.

[88] T. Kamalakis and T. Sphicopoulos. Analytical expressions for the resonant frequencies and modal fields of finite coupled optical cavity chains. IEEE J. Quant. El., 41(1):1419 - 1425, 2005.

[89] A.L. Reynolds, U. Peschel, F. Lederer, P.J. Roberts, T.F. Krauss, and P.J.L. de Maagt. Coupled defects in photonic crystals. IEEE Trans. MTT, 49(10): 1860 - 1867, 2001.

[90] H. Haus and W. Huang. Coupled-mode theory. Proc. IEEE, 79(10):15051518, 1991.

[91] D. G. Hall and B. J. Thompson, editors. Selected Papers on Coupled-Mode Theory in Guided-Wave Optics, volume MS 84 of SPIE Milestone Series. SPIE Optical Engineering Press, Bellingham, Washington USA, 1993.

[92] W. P. Huang. Coupled mode theory for optical waveguides: an overview. Journal of the Optical Society of America A, 11(3):963-983, 1994.

[93] M. Maksimovic, M. Hammer, and E. van Groesen. Field representation for optical defect resonances in multilayer microcavities using quasi-normal modes. Optics Comm., 281:1401-1411, 2008.

[94] Françoise Tisseur and Karl Meerbergen. The Quadratic Eigenvalue Problem. SIAM Rev., 43(2):235-286, 2001.

[95] Vladimir M. Shalaev. Electromagnetic Properties of Small-Particle Composites. Phys. Rep., 272(61), 1996.

[96] W. S. Weiglhofer and A. Lakhtakia (editors). Introduction to Complex Mediums for Optics and Electromagentics. SPIE Press, 2003.

[97] G.V. Eleftheriades and K.G. Balmain (editors). Negative-Refraction Metamaterials: Fundamental Principles and Applications. John Wiley and Sons and IEEE Press, 2005.

[98] D. R. Smith, Willie J. Padilla, D. C. Vier, S. C. Nemat-Nasser, and S. Schultz. Composite medium with simultaneously negative permeability and permittivity. Phys. Rev. Lett., 84(4184), 2000.

[99] J. B. Pendry. Negative refraction makes a perfect lens. Phys. Rev. Lett., 85(3966), 2000. 
[100] D. R. Smith, D. Schurig, R. Rosenbluth, S. Schultz, S.A. Ramakrishna, and J. B. Pendry. Limitations on subdiffraction imaging with a negative refractive index slab. Appl. Phys. Lett., 82:1506108, 2003.

[101] N. Fang, H. Lee, C. Sun, and X. Zhang. Sub-diffraction limited optical imaging with a silver superlens. Science, 308:534-537, 2005.

[102] D. O. S. Melville and R. J. Blaikie. Super-resolution imaging through a planar silver layer. Opt. Expr., 13:2127- 2134, 2005.

[103] A. V. Kildishev et al. Negative refractive index in optics of metaldielectric composites. J. Opt. Soc.Am. B, 23:423433, 2006.

[104] S. Linden, C. Enkrich, G. Dolling, M.W. Klein, J. Zhou, T. Koschny, C.M. Soukoulis, S. Burger, F. Schmidt, and M. Wegener. Photonic Metamaterials: Magnetism at Optical Frequencies. IEEE J. Sel. Top. in QE, 12:1097-1105, 2006.

[105] Costas M. Soukoulis, Stefan Linden, and Martin Wegener. Negative Refractive Index at Optical Wavelengths. Science, 315:47, 2007.

[106] R.W. Ziolkowski and E. Heyman. Wave propagation in media having negative permittivity and permeability. Phys. Rev. E, 64(056625), 2001.

[107] L. Feng, X-P. Liu, M-H. Lu, and Y-F. Chen. Phase compensating effect in left-handed materials. Phys. Lett. A, 332(5-6):449-455, 2004.

[108] D. Bria, B. Djafari-Rouhani, A. Akjouj, L. Dobrzynski, J. P. Vigneron, E. H. El-Boudouti, and A. Nougaoui. Band structure and omnidirectional photonic band gap in lamellar structures with left-handed matrials. Phys. Rev. E, 69(066613), 2004.

[109] H. Daninthe, S. Foteinopoulou, and C.M. Soukoulis. Omni-reflectance and enhanced resonant tunneling from multilayers containing left-handed materials. Photon. and Nanostr., 4(3):123-131, 2006.

[110] Liang $\mathrm{Wu}$, Sailing He, and Long Chen. On unusual narrow transmission bands for a multi-layered periodic structure containing left-handed materials. Optics Express, 11(11):1283-1290, 2003.

[111] Josef Honerkamp. Statistical Physics: An Advanced Approach with Applications. Springer-Verlag, 1998.

[112] C. Luo, A. Narayanaswamy, G. Chen, and J. D. Joannopoulos. Thermal radiation from photonic crystals: A direct calculation. Phys. Rev. Lett., 93(213905), 2004.

[113] P. B. Abdallah. Thermal antenna behavior for thin-film structures. J.Opt. Soc. Am. A, 21(7):1368-1371, 2004. 
[114] F. O Sullivan, I. Celanovic, N. Jovanovic, J. Kassakian, S. Akiyama, and K. Wada. Optical charactristics of one dimensional si-sio2 photonic crystals for thermophotovoltaic applications. J. App. Phys, 97(033529), 2005.

[115] B. J. Lee and Z. M. Zhang. Design and fabrication of planar multilayer structures with coherent thermal emission characteristics. J. App. Phys, 100(063529), 2006.

[116] M. Maksimovic and Z. Jaksic. Modification of thermal radiation by periodical structures containing negative refractive index metamaterials. Phys. Lett. A, 342(5-6):497-503, 2005.

[117] M. Maksimovic and Z. Jaksic. Emittance and absorptance tailoring by negative refractive index metamaterial-based cantor multilayers. J. Optics A: Pure and App. Opt., 8(3):352-362, 2006.

[118] F. F. de Medeiros, E. L. Albuquerque, M. S. Vasconcelos, and P. W. Mauriz. Thermal radiation in quasiperiodic photonic crystals with negative refractive index. J. Phys.: Condens. Matter, 19:496212, 2007.

[119] M. Maksimovic and Z. Jaksic. A Fibonacci-type quasi-periodic nanocomposite filters containing negative index metamaterials for emittance tailoring. In Proc. of 1st International Workshop on Nanoscience and Nanotechnology IWON, Belgrade, pages 147-156, 2005.

[120] M. Maksimovic, M. Hammer, and Z. Jaksic. Thermal radiation antennas made from multilayer structures containing negative index metamaterials. Proc. SPIE, 6896:689605, 2008.

[121] J. Li, D. Zhao, and Z. Liu. Zero-n photonic band gap in quasiperiodic stacking of positive and negative refractive index metamaterials. Phys. Lett. A, 332:461-468, 2004.

[122] J. Gerardin and A. Lakhtakia. Spectral response of Cantor multilayers made of materials with negative refractive index. Phys. Lett. A, 301:377-381, 2002.

[123] R. Merlin. Structural and electronic properties of nonperiodic superlattices. IEEE J. Quant. Electr., 24:1791-1798, 1988.

[124] Nian-Hua Liu. Propagation of light waves in Thue-Morse dielectric multilayers. Phys. Rev. B, 55(6):3543-3547.

[125] H. Lei, J. Chen, G. Nouet, S. Feng, Q. Gong, and X. Jiang. Photonic band gap structures in the Thue-Morse lattice. Phys. Rev. B, 75:205109, 2007.

[126] D. C. Vier S. C. Nemat-Nasser D. R. smith, Willie J. Padilla and S. Schultz. A composite medium with simultaneously negative permeability and permittivity. Phys. Rev. Lett., 84:4184, 2000. 
[127] Shawn-Yu Lin, J. G. Fleming, E. Chow, Jim Bur, K. K. Choi, and A. Goldberg. Enhancement and suppression of thermal emission by a threedimensional photonic crystal. Phys. Rev. B, 62(4):R2243-R2246, 2000.

[128] Peter W. Milonni and G. Jordan Maclay. Quantized-field description of light in negative-index media. Optics Comm., 228(1-3):161-165, 2003.

[129] Ho Trung Dung, Stefan Yoshi Buhmann, Ludwig Knöll, Dirk-Gunnar Welsch, Stefan Scheel, and Jürgen Kästel. Electromagnetic-field quantization and spontaneous decay in left-handed media. Phys. Rev. A, 68(4):043816, 2003.

[130] J. Kästel and M. Fleischhauer. Suppression of spontaneous emission and superradiance over macroscopic distances in media with negative refraction. Phys. Rev. A, 71:01180, 2005.

[131] J. Kästel and M. Fleischhauer. Quantum electrodynamics in media with negative refraction. Laser Phys., 15:1-11, 2005.

[132] Jing-Ping Xu, Nian-Hua Liu, , and Shi-Yao Zhu. Spontaneous emission field of a two-level atom embedded in one-dimensional left-handed- and right-handed-material photonic crystals. Phys. Rev. E, 73:016604, 2006.

[133] D. L. Chan, M. Soljacic, and J. D. Joannopoulos. Thermal emission and desig in one-dimensional periodic metallic photonic crystal slabs. Phys. Rev. E, 74(016609), 2006.

[134] C. Sibilia, P. Masciulli, and M. Bertolotti. Optical properties of quasiperiodic (self-similar) structures. Pure Appl. Opt., 7:383-391, 1998.

[135] C. Sibilia, M. Scalora, M. Centini, M. Bertolotti, M. J. Bloemer, and C. M. Bowden. Electromagnetic properties of periodic and quasi-periodic onedimensional, metallodielectric photonic band gap structures. J.Opt. A: Pure Appl. Opt., 1:490-494, 1999.

[136] A. V. Lavrinenko, S. V. Zhukovsky, K. S. Sandomirskii, and S. V. Gaponenko. Propagation of classical waves in non-periodic media: Scaling properties of an optical Cantor filter. Phys. Rev. E, 65:036621/1-8, 2002.

[137] X. Sun and D. L. Jaggard. Wave interaction with generalized Cantor bar fractal multilayers. J. Appl. Phys., 70:2500-2507, 1991.

[138] S. V. Zhukovsky, A. V. Lavrinenko, and S. V. Gaponenko. Spectral scalability as a result of geometrical self-similarity in fractal multilayers. Europhys. Lett., 66:455-461, 2004.

[139] A. Lakhtakia, K. E. Weaver, and A. Verma. Transmission through Cantor filters revisited. Optik, 113:510-512, 2002. 
[140] M. S. Vasconcelos, P. W. Mauriz, F. F. de Medeiros, and E. L. Albuquerque. Photonic band gaps in quasiperiodic photonic crystals with negative refractive index. Phys. Rev. B, 76:165117, 2007. 


\section{List of publications}

\section{Journals}

- M. Maksimovic, M. Hammer, E. van Groesen, Field representation for optical defect resonances in multilayer microcavities using quasi-normal modes, Optics Communications, Vol. 281, pp. 1401-1411, (2008)

- M. Maksimovic, Z. Jaksic, Transmission spectra of Thue-Morse multilayers containing negative index metamaterials, Acta Physica Polonica A,Vol. 112, No. 5, pp. 1055-1060, (2007)

- M. Maksimovic, Z. Jaksic, Emittance and absorptance tailoring by negative refractive index metamaterial-based Cantor multilayers, Journal of Optics A: Pure and Applied Optics , Vol. 8, No. 3, pp. 355-362, (2006)

- M. Maksimovic, Z. Jaksic, Modification of thermal radiation by periodical structures containing negative refractive index metamaterials, Physics Letters A, Vol. 342, No. 5-6, pp. 497-503, (2005)

\section{Contribution to conference proceedings printed in full}

- M. Maksimovic, M. Hammer, E. van Groesen, Coupled optical defect microcavities in $1 D$ photonic crystals and quasi-normal modes, Integrated Optics: Devices, Materials, and Technologies XII, Christoph M. Greiner; Christoph A. Waechter, Editors, Proceedings of SPIE, Vol. 6896,689603, (2008)

- M. Maksimovic, M. Hammer, Z. Jaksic, Thermal radiation antennas made of multilayer structures containing negative index metamaterials, Integrated Optics: Devices, Materials, and Technologies XII, Christoph M. Greiner; Christoph A. Waechter, Editors, Proceedings of SPIE, Vol. 6896,689605, (2008) 


\section{Posters}

- M. Maksimovic, M. Hammer, E. van Groesen, Coupled optical defect cavities in finite 1-D photonic crystals and quasi-normal modes, NanoNed / MinacNed Symposium, Nijmegen, The Netherlands; book of abstracts 105, (2007)

- M. Maksimovic, M. Hammer, E. van Groesen, Field representations for optical defect microcavities in $1 D$ grating structures using quasi-normal modes, XVI International Workshop on Optical Waveguide Theory and $\mathrm{Nu}$ merical Modeling, Copenhagen, Denmark; book of abstracts 11, (2007)

- M. Maksimovic, Z. Jaksic, Wave propagation in finite one-dimensional Thue - Morse superlattices conatining negative index metamaterials, Inter. School and Conference on Optics and Optical Materials ISCOM, Belgrade, Serbia; book of abstracts 122, (2007)

- M. Maksimovic, M. Hammer, E. van Groesen, Analysis of optical defect cavities in $1 D$ grating structures with quasi-normal mode theory, NanoNed / MicroNed Symposium II, Eindhoven, The Netherlands; book of abstracts 170, (2006) 


\section{Summary and outlook}

Theoretical research in optics may be divided in two distinctive but well connected general directions. The first deals with developing new or improving existing mathematical models to describe relevant physics. The second aims to predict new phenomena or applications using established models and techniques. This thesis touches both directions through the study of resonances in optical multilayers.

Optical multilayers are structures periodic in their properties in one direction. They have been longstanding subject of investigation. Nonetheless, both fundamental and applied research in multilayer optics is still important due to the relevance of multilayer structures for optical systems. The introduction of specific defects in otherwise periodic configurations enables effective tailoring of the optical transmission properties. Equally important, novel materials give additional degrees of freedom for the implementation of desired functionalities as well as the exploration of new physical phenomena. Besides, knowledge gained from an investigation of multilayer structures may serve as a basis for the interpretation and the qualitative understanding of higher dimensional optical structures.

We consider the open and finite nature of a specific class of multilayer structures by directly characterizing their resonance properties via an investigation of the quasi-normal mode spectrum. Quasi-normal modes are eigenfunctions that appear as solutions of the eigenvalue problem for open structures. They are field profiles representing damped oscillations of the open optical system after an initial excitation is withdrawn.

Specifically, we are interested in the field representation and in perturbation techniques for defect resonances of defect based one-dimensional photonic crystal. First, a recently developed QNM expansion method applied for the solution of the scattering problem is briefly reviewed to model examples of the optical defect microcavities in periodic multilayers. Second, we propose a novel and constructive way of connecting a quasi-normal mode description to transmission resonance properties of optical defect microcavities in 1D multilayer structures.

Our approach is meant specifically for approximations of the defect induced transmission modes existing in the bandgap of otherwise periodical structures. It relies on a variational principle for the field representation of the field profiles in the transmission problem. A field template with a mirror field and the most relevant QNMs enables very accurate field and spectral transmission approximations that agree excellently with the TMM reference. Moreover, method allows to exam- 
ine directly the resonance nature of the transmission response in cases where it is very hard to establish this from exact solutions of the transmission problem, such as provided by the TMM method. The approach quantifies directly the physical viewpoint, where the defect cavities are regarded as externally forced oscillators.

Furthermore, a variational principle for QNMs allows to approximate the eigenfrequencies and QNMs of composite multiple cavity structures by eigenfrequencies and QNMs of simpler structures. Hence, a form of coupled mode theory for finite, open 1-D PC structures is proposed, that uses directly the most relevant QNMs. Closely related, an expression for a first order perturbation correction of the complex eigenfrequencies is derived by means of variational restriction.

We analyzed a series of characteristic examples of multiple cavity structures and were able to point out characteristic features in the composite structures as originating from simpler structures. The results suggest that the notion of the photonic crystal molecules can be founded on the analysis of QNM spectra rigged with the variational approximation method. Therefore, the QNM analysis offers a resourceful method for the interpretation of complex phenomena associated with the resonance properties in 1-D PC structures. Numerical examples suggest that the method is valid for single and multiple cavity structures in both symmetric and nonsymmetric layer arrangements and both weak and strong couplings between defects.

Further research might explore the application of QNMs analysis on the photonic crystal heterostructures, composed from different types of complex unit cells with different periods. The investigation of the photonic crystal molecule concept should include photonic crystal atoms as truncated non-periodic superlattices. Furthermore, provided that suitable QNM basis fields can be made available by analytical or numerical means, generalizations to two or three spatial dimensions could be based on the functional representations of the frequency domain Maxwell equations. Alternative formulations of the QNM problem using the (first order) Maxwell equations directly rather than the (second order) Helmholtz equation are an interesting possibility.

A second class of problems that we address concerns multilayer structures incorporating negative index metamaterials, which are artificial composites with subwavelength features and negative real part of the refractive index of the homogenized structure. We use the Transfer Matrix Method, as outlined in chapter 1, as mathematical method for numerical computations and analysis.

Novel properties of the bandgap structure and transmission spectra can be obtained by the introduction of NIMs in the construction of the multilayers. Key mechanism responsible for novel properties is the phase compensation, the partial or full removal of the phase shift of the wave propagating through a NIMcontaining multilayer.

First we analyzed transmission spectra for aperiodic Thue-Morse multilayers composed from alternating layers with positive and negative refractive indices. In contrast to other non-periodic NIM-containing multilayers where self-similar 
and scalable spectra occurred for higher generations, for Thue-Morse multilayers these spectral properties do not appear, even in the dispersionless and lossless case. However, Thue-Morse multilayers exhibit transmission resonances located at the midgap frequency of the periodic multi-layer. The field distribution at the resonance frequency suggests a common origin in both all-PIM and NIM-containing multilayers connected with the special order of layers. We demonstrated by direct computation that the strong material dispersion in the NIM materials may effectively diminish the influence of the aperiodic order and the phase compensation over a large frequency range. Only the prospect of weak material dispersion in the NIM leads to a potential for the application of the optical phenomena associated with aperiodic order.

We apply passive NIM-containing multilayers to tailor the spectral and angular emittance/absorptance distributions of a thick absorbing substrate. On the basis of the transfer matrix method and of the Kirchhoffs law for thermal radiation, we analyze realistic finite structures that comprise NIM-containing multilayers. Dispersion and losses in the NIM part are taken into account. First, we analyze finite periodic multilayers. Our results show that structures containing NIM show large influence to the thermal radiation spectrum. The suppressed region of thermal radiation is wider than in usual all-PIM structures, and the spectral characteristics more flat, i.e. without sharp oscillations typical for the all-dielectric case. It can be also seen that the thermal radiation absorbtance/emittance is less dependent on the angle of the incident radiation. Second, our investigations were dedicated to pre-fractal multilayers of triadic Cantor type containing dispersive and lossy negative index media. As for other NIM- containing multilayers the dependence of the spectral transmission on the spatial angle is much weaker than for conventional non-periodic multilayers, while the sequential splitting gives rise to multiple narrow transmission peaks, which has an applicative potential itself.

The theoretical results suggest that a NIM-containing multilayer on top of an absorbing substrate implements the concept of thermal radiation antenna, i.e. a system that enables both spectral and directional selectivity of the thermal power spectrum emitted by some material object.

Some special features not achievable by conventional all-positive index structures may arise when the filtering structure containing both NIM and PIM strata is designed to operate within the zero-n bandgap regime. Although, the zero-n bandgap regime requires additional analysis, in this mode of operation it might be possible to render rejection bands which are wide both in the spectral and the angular domain, or, alternatively, ultra-narrow pass-bands with angular superselectivity. With the prospect of the NIMs operating for optical frequencies and with controllable intrinsic dispersion and absorption properties, NIM-containing structures can be seen as a promising design choice for thermal radiation antennas. 


\section{Samenvatting}

Theoretisch onderzoek in de optica kan in twee verschillende maar duidelijk gerelateerde hoofdrichtingen worden opgedeeld. De eerste houdt zich bezig met het ontwikkelen van nieuwe of het verbeteren van bestaande wiskundige modellen om relevante natuurkunde te beschrijven. De tweede richt zich op het voorspellen van nieuwe fenomenen of toepassingen, gebruik makend van bestaande modellen en technieken. Dit proefschrift raakt aan beide richtingen door de studie van resonanties in optische multilagen.

Optische multilagen zijn strukturen die periodiek zijn in hun eigenschappen in een richting. Ze zijn al sinds lange tijd onderwerp van onderzoek. Echter, zowel fundamenteel als toegepast onderzoek naar optica in multilagen is nog altijd van belang vanwege de relevantie van multilaagsstrukturen voor optische systemen. De introductie van specifieke defecten in verder periodieke strukturen maakt het mogelijk de optische transmissie-eigenschappen op effectieve wijze aan te passen. Net zo belangrijk is het feit dat nieuwe materialen extra vrijheidsgraden geven voor zowel het implementeren van gewenste functionaliteiten als het verkennen van nieuwe natuurkundige fenomenen. Bovendien kan de kennis die wordt gegenereerd bij het onderzoek naar multilaagsstrukturen als basis dienen voor het interpreteren en kwalitatief begrijpen van hoger-dimensionale optische strukturen.

Wij beschouwen het open en eindige karakter van een specifieke klasse van multilaagsstrukturen door hun resonantie-eigenschappen direkt te karakteriseren via het onderzoek naar hun 'quasi-normal mode' (QNM) spectrum. Quasi-normal modes zijn eigenfuncties die verschijnen als oplossingen van het eigenwaardeprobleem voor open strukturen. Dit zijn veldprofielen die de gedempte oscillaties vertegenwoordigen van het open optische systeem nadat een aanvankelijke excitatie is weggenomen.

In het bijzonder zijn we geinteresseerd in de veldrepresentatie van en in verstoringstechnieken voor defect-resonanties van een-dimensionale fotonische kristallen gebaseerd op defecten. Als eerste wordt een recent ontwikkelde QNM expansie methode, toegepast op het oplossen van het verstrooiingsprobleem, kort bekeken om voorbeelden van optische defect-microtrilholtes in 1-D multilaagsstrukturen de modelleren. Ten tweede stellen we een nieuwe en constructieve manier op om een verbinding aan te brengen tussen een quasi-normal mode beschrijving en de transmissie resonantie eigenschappen van optische defect-microtrilholtes in 1-D multilaagsstrukturen. 
Onze aanpak is in het bijzonder bedoeld voor benaderingen van transmissie modes die door een defect geinduceerd worden, gelegen in de bandgap van verder periodieke strukturen. $\mathrm{Zij}$ is gestoeld op een variationeel principe voor de veldrepresentatie van de veldprofielen in het transmissieprobleem. Een veldsjabloon met een spiegelveld en de meest relevante QNM's maakt zeer nauwkeurige veld- en spectrale transmissiebenaderingen mogelijk, die uitstekend overeenkomen met de TMM referentie. Behalve dit maakt de methode het mogelijk het resonantiekarakter van de transmissierespons direct te inspecteren, in gevallen waarin het erg moeilijk is om deze informatie te extraheren uit exacte oplossingen van het transmissieprobleem, zoals verkregen uit de TMM methode. De aanpak quantificeert direct het natuurkundige oogpunt, waarin de defect-trilholtes als extern gedreven oscillatoren worden gezien.

Verder staat een variationeel principe voor QNM's het toe om de eigenfrequenties en QNM's van samengestelde strukturen van meerdere trilholtes te karakteriseren met behulp van de eigenfrequenties en QNM's van eenvoudiger strukturen. Dus wordt een vorm van een gekoppelde-mode theorie voor eindige, open 1D PC strukturen voorgesteld, die de meest relevante QNM's direct gebruikt. Dicht hieraan gerelateerd wordt een uitdrukking voor een eerste orde verstoringscorrectie van de complexe eigenfrequenties afgeleid door middel van variationele restrictie.

We hebben een aantal karakteristieke voorbeelden van meerdere-trilholte strukturen geanalyseerd en waren in staat aan te tonen dat karakteristieke kenmerken in de samengestelde strukturen aan eenvoudiger strukturen ontspringen. Dit resultaat suggereert dat het idee van fotonisch kristal moleculen gebaseerd kan worden op de analyse van QNM spectra, opgetuigd met de variationele benaderingsmethode. De QNM analyse levert dus een vernuftige methode voor de interpretatie van de complexe fenomenen geassocieerd met de resonantie-eigenschappen in 1-D PC strukturen. Numerieke voorbeelden suggereren dat de methode geschikt is voor enkele en meerdere trilholte strukturen in zowel symmetrische als niet-symmetrische ordening van lagen en voor zowel zwakke als sterke koppeling tussen defecten.

Verder onderzoek zou de toepassing kunnen verkennen van de analyse van QNMs op fotonisch kristal heretostrukturen, gevormd uit verschillende types complexe eenheidscellen met verschillende periodes. Het beschouwen van het fotonische kristal molekuul concept zou fotonisch kristal atomen als afgekapte niet- periodieke superroosters moeten bevatten. Bovendien, aangenomen dat geschikte QNM basisvelden beschikbaar kunnen worden gemaakt op analytische of numerieke manier, zouden generalisaties naar twee of drie ruimtelijke dimensies kunnen worden gebaseerd op de functionaalrepresentaties van de Maxwellvergelijkingen in het frequentiedomein. Alternatieve formuleringen van het QNM probleem direct gebruik makend van de (eerste orde) Maxwellvergelijkingen in plaats van de (tweede orde) Helmholtz vergelijking zijn een interessante mogelijkheid.

Een tweede klasse problemen waar we ons op richten houdt zich bezig met multilaagsstrukturen waarin negatieve-index metamaterialen zijn opgenomen, wat kunstmatig samengestelde materialen zijn met componenten kleiner dan de golflengte, waarbij het reele deel van de brekingsindex van de gehomogeniseerde struktuur 
negatief is. We gebruiken de Transfer Matrix Method (TMM), zoals omschreven in Hoofdstuk 1, als wiskundige methode voor de numerieke berekeningen en analyse.

Nieuwe eigenschappen van de bandgap struktuur en transmissiespectra kunnen verkregen worden door introductie van Negatieve Index Materialen (NIM's) in de constructie van de multilagen. Het sleutelmechanisme dat verantwoordelijk is voor de nieuwe eigenschappen is fase compensatie, de gedeeltelijke of volledige verwijdering van de fase-verandering van de golf die door een multilaag met NIM propageert.

Eerst hebben we transmissiespectra geanalyseerd van aperiodieke Thue-Morse multilagen gevormd van lagen met om en om positieve en negatieve brekingsindices. In tegenstelling tot andere niet-periodieke multilaagsstrukturen met NIM waarin zelf-gelijkvormige en schaalbare spectra optraden voor hogere generaties, verschijnen deze spectrale eigenschappen niet voor Thue-Morse multilagen, zelfs in het dispersie- en verliesvrije geval. Echter, Thue-Morse multilagen vertonen transmissieresonanties gelegen op de mid-gap frequentie van de periodieke multilaag. De veldverdeling op de resonantiefrequentie suggereert een gelijke oorsprong in zowel volledig PIM multilagen alsook multilagen met NIM, verbonden met de speciale ordening van de lagen. We hebben door directe berekening laten zien dat de sterke materiaaldispersie in de NIM's de invloed van zowel de aperiodieke ordening als de fasecompensatie over een groot frequentiegebied effectief vermindert. Enkel de verwachting van lage materiaaldispersie in het NIM leidt tot een potentieel voor de toepassing van de optische fenomenen geassiocieerd met aperiodieke orde.

We passen passieve multilagen met NIM's toe op het aanpassen van de spectrale en radiele emittantie / absorptie distributies van een dik absorberend substraat. Op basis van de TMM en van Kirchhoff's wet van warmtestraling, analyseren we realistische eindige strukturen die NIM-bevattende multilagen bevatten. Dispersie en verlies in het NIM deel wordt meegenomen. Ten eerste analyseren we eindige periodieke multilagen. Onze resultaten laten zien dat strukturen met NIM een grote invloed op het warmtestralingsspectum laten zien. Het onderdrukte gebied van warmtestraling is groter dan in gebruikelijke volledig-PIM strukturen, en de spectrale karakteristiek is vlakker, dus zonder de scherpe oscillaties die typisch zijn voor het puur dielectrische geval. Het is eveneens te zien dat de absorptie / emittantie van de warmtestraling minder afhankelijk is van de hoek van inval van de straling. Ten tweede waren onze onderzoeken gericht op pre-fractal multilagen van triadisch Cantor type, met dispersieve en niet-verliesvrije negatieve-brekingsindex media. Net als in andere multilagen met NIM is de invloed van de ruimtelijke hoek op de spectrale transmissie veel zwakker dan in conventionele niet-periodieke multilagen, terwijl de opeenvolgende splitsing aanleiding geeft tot meerdere smalle transmissiepieken, wat op zich al mogelijke toepassingen heeft.

De theoretische resultaten suggereren dat een multilaag met NIM bovenop een absorberend substraat het concept van een warmtestralingsantenne implementeert, dus een systeem is dat zowel spectrale als directionele selectiviteit mogelijk maakt 
van het warmtestralingsspectrum dat uitgezonden wordt door een object.

Enkele bijzondere kenmerken die niet haalbaar zijn met conventionele puur positieve-index strukturen kunnen ontstaan wanneer de filterstruktuur die zowel NIM and PIM lagen bevat is ontworpen om binnen het nul-n bandgap regime te functioneren. Hoewel er extra onderzoek nodig is in het nul-n bandgap regime, zou het mogelijk kunnen zijn om in dit werkingstype afwijzingsbanden te genereren die zowel in het spectrale als in het ruimtehoekdomein breed zijn of, daarentegen, ultra-smalle transmissiebanden met super ruimtehoekselectiviteit. Met de verwachting van NIM's die werken op optische frequenties en met selecteerbare intrinsieke dispersie- en absorptieeigenschappen, kunnen strukturen met NIM's worden beschouwd als een veelbelovende ontwerpkeuze voor warmtestralingsantennes. 


\section{Acknowledgement}

This thesis is a product of four years journey into the filed of optics. It spanned two countries and two different institutions where many people contributed directly or indirectly to the work presented in this thesis.

Last two years I have been with the Applied Analysis and Mathematical Physics Group in the Department of Applied Mathematics, University of Twente. Research concerning resonances in optical structures was embedded in the NanoNed / Nanophotonics Flagship project "Optical switching by NEMS-actuated resonator arrays, modeling and simulation tools".

Previously, I was with the Institute of Microelectronic Technologies and Single Crystals, Division of Institute of Chemistry, Technology and Metallurgy, University of Belgrade. Part of research activities concerning negative index metamaterials that I carried out during that period is a part of this thesis.

I would like to express sincere gratitude to my promotor Brenny van Groesen for giving me opportunity to work in his group and for his support to work presented in this thesis. Also, my gratitude and many thanks goes to my co-promotor Manfred Hammer for his support, many valuable discussions, his insistence on preciseness and especially for all the hard work he did to improve my writings. Without Manfred this thesis would not be possible.

I am very much indebted to Zoran Jakšić for many things in both professional and private life. Our collaboration enabled me to start my scientific work four years ago. Many discussions and brainstorming sessions will be always in my memory.

I also would like to thank the other members of the graduation committee: $\mathrm{H}$. J. W. M. Hoekstra, B.J. Hoenders, L. Kuipers and H.P. Urbach for their willingness to be in the graduation committee and for constructive suggestions and comments on this thesis.

Discussions during the "optics meetings" and comments from Manfred, Remco, Alyona, Hugo and Rene are very much appreciated. Special thanks goes to Remco (who also translated summary of the thesis in Dutch) and Alyona for all the help I received from them. Also, I wish to express my sincere gratitude to all members of AAMP and NACM groups for a pleasant time I have spent at UT. Thanks to Marielle for all help concerning administration stuff.

Many thanks goes to my office mates at UT: Arek, Lars, Tim, Ivan, Henk, Joris and Fedderik; and also to all people in Belgrade that I have shared space with in "cvećara". 
I am grateful to my parents and family for their support and love and for believing in my success.

I owe everything to Sanja, the most important person in my life. Without you my life would be empty. Your love is only what matters and only what I need.

Milan Maksimović

15. March 2008,

Enschede, The Netherlands 